\title{
In Silico Comparison of Photons versus Carbon lons in Single Fraction Therapy of Lung Cancer
}

In Silico Vergleich der Lungen Krebstherapie mit Photonen und Kohlenstoff Ionen bei Einzeitbestrahlung

Zur Erlangung des Grades eines Doktors der Naturwissenschaften (Dr. rer. nat.) genehmigte Dissertation von Dipl.-Phys. Kristjan Anderle aus Jesenice, Slowenien Darmstadt 2016 - D 17

Fachbereich Physik

1. Gutachten: Prof. Dr. Marco Durante

2. Gutachten: Prof. Dr. Thomas Aumann 
In Silico Comparison of Photons versus Carbon Ions in Single Fraction Therapy of Lung Cancer In Silico Vergleich der Lungen Krebstherapie mit Photonen und Kohlenstoff Ionen bei Einzeitbestrahlung

Genehmigte Dissertation von Dipl.-Phys. Kristjan Anderle aus Jesenice, Slowenien

1. Gutachten: Prof. Dr. Marco Durante

2. Gutachten: Prof. Dr. Thomas Aumann

Tag der Einreichung: 14. Juni 2016

Tag der Prüfung: 4. Juli 2016

Darmstadt - D 17 


\section{Abstract}

Stereotactic body image guided radiation therapy (SBRT) shows excellent results for the local control of early stage lung cancer. However, not all patients are eligible for SBRT, and advanced stage treatment is less successful and often associated with severe side effects. Scanned carbon ion therapy (PT) can deliver more conformal dose likely benefiting these patient groups.

Therefore an in silico trial was conducted on early and advanced stage patients to identify potential advantages of PT. The patients were treated with SBRT at Champalimaud Center for the Unknown, Lisbon (Portugal). PT plans were simulated on 4DCTs, and rescanning was investigated for motion mitigation in 4D-dose calculations. A dedicated strategy for $4 \mathrm{D}$ intensity modulated particle therapy (IMPT) was developed and applied for advanced stage patients with multiple lesions. For clinically valid and reliable results the deformable image registration necessary for 4D-dose calculation - a quality assurance tool was developed and applied in the study.

The results showed that target coverage was comparable in SBRT and PT, while PT delivered significantly lower doses to most critical structures, especially the heart, lungs, and esophagus. A highly complex case of advanced stage lung cancer could be treated in a single fraction of 24 Gy with PT, while SBRT could not deliver the full ablative dose treatment due to an excessive heart dose. The mean heart dose was reduced from $10 \mathrm{~Gy}$ to $0.8 \mathrm{~Gy}$ with PT for this specific patient. 



\section{Zusammenfassung}

Stereotaktische Radiochirurgie (SBRT) zeigt exzellente Ergebnisse für die lokale Kontrolle von Lungenkrebs im Frühstadium. Viele Patienten sind allerdings nicht für die SBRT geeignet, und die Behandlung von späteren Stadien führt oft zu schweren Nebenwirkungen. Die Bestrahlung mit gescanntem Kohlenstoff (PT) ermöglicht eine konformere Dosisapplikation, wovon gerade diese Patientengruppen profitieren könnten.

Eine in silico Studie an Lungenkrebspatienten in frühen und späten Stadien wurde durchgeführt, um mögliche Vorteile von PT zu untersuchen. Die Patienten wurden am Champalimaud Center for the Unknown, Lissabon (Portugal) mit SBRT behandelt. PT Pläne wurden auf 4DCTs simuliert und zur Bewegungskompensation wurde Rescanning in 4D-Dosisberechnungen untersucht. Eine dedizierte Strategie für 4D Intensitäts-modulierte Partikeltherapie (IMPT) wurde entwickelt und für Patienten im fortgeschrittenem Stadium mit mehreren Läsionen eingesetzt. Für klinisch valide und verlässliche Ergebnisse wurde für Nicht-rigide Bildregistrierung - für die 4D-Dosisberechnung unerlässlich eine Strategie zur Validierung und Qualitätssicherung entwickelt.

Es ergab sich eine vergleichbare Dosisabdeckung der Ziele für PT und SBRT, mit PT konnte die Dosisbelastung fast aller Risikoorgane aber signifikant gesenkt werden, insbesondere des Herzens, der Lunge und der Speiseröhre. In einem besonders komplexen Fall von Stufe IV Lungenkrebs konnte PT alle 5 Läsionen mit der vollen Dosis von 24 Gy abdecken, während dies mit SBRT durch die zu hohe Herzdosis nicht möglich war - die mittlere Herzdosis konnte mit PT trotz voller Zieldosis von 10 Gy auf 0.8 Gy reduziert werden. 



\section{Publications related to this work}

\section{Article in revision}

Anderle K., Stroom J., Pimentel J., Greco C., Durante M. and Graeff C.: In Silico Comparison of Photons versus Carbon Ions in Single Fraction Therapy of Lung Cancer; revision under review in Phys.Med., submitted in March 2016

\section{GSI scientific report}

Anderle K., Stroom J., Pimentel J., Greco C., Durante M. and Graeff C.: An in silico Trial of X-rays vs Carbon Ions in Single Shot Lung Cancer Therapy; GSI Scientific Report 2013, 228

\section{Conference contributions}

Anderle K., Stroom J., Pimentel J., Greco C., Durante M. and Graeff C. (2013). An in silico Comparison of Scanned Carbon Ion vs. SBRT Single Dose Treatment of Metastatic Lung Cancer; $4 D$ Treatment planning workshop. Poster presentation

Anderle K., Stroom J., Pimentel J., Greco C., Durante M. and Graeff C. (2014). An in silico Comparison of Single Fraction Scanned Carbon Ion vs. SBRT in Metastatic Lung Cancer; ESTRO. Poster presentation

Anderle K., Stroom J., Pimentel J., Greco C., Durante M. and Graeff C. (2015). In Silico Trial of Photons versus Carbon Ions in Single Fraction Therapy of Lung Cancer; PTCOG. Poster presentation

Anderle K., Stroom J., Pimentel J., Greco C., Durante M. and Graeff C. (2015). In Silico Trial of Photons versus Carbon Ions in Single Fraction Therapy of Lung Cancer; DGMP. Poster presentation

Prall M., Hild S., Anderle K. and Graeff C. (2016). Treatment Planning with an Adaptive Dose Grid; PTCOG. Poster presentation

Eichhorn A., Hild S., Anderle K. and Graeff C. (2016). Speed-up of plan delivery for intensity modulated particle therapy by splitting iso-energy slices according to beam intensity levels; PTCOG. Poster presentation 
Vieira S., Stroom J., Anderle K., Salas B., Pimentel N. and Greco C. (2016). Validation of freeware-based midventilation CT calculation for upper abdominal cancer patients; ESTRO. Poster presentation 


\section{Contents}

Motivation

$\begin{array}{ll}\text { 1. Introduction - research background and fundamentals } & 13\end{array}$

1.1. Radiotherapy . . . . . . . . . . . . . . . . . . . . . 13

1.2. Physical and biological basics of radiotherapy . . . . . . . . . . . 14

1.3. Lung cancer . . . . . . . . . . . . . . . . . . . . 39

2. Deformable Image Registration and Validation 43

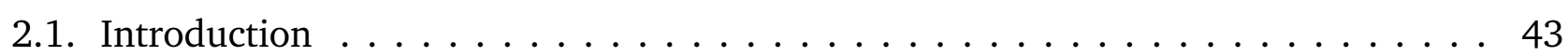

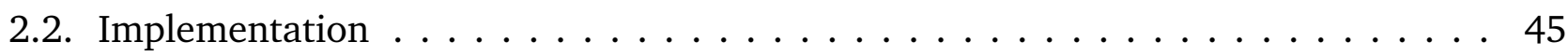

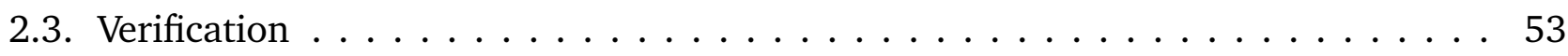

2.4. Summary and Discussion $\ldots \ldots \ldots \ldots \ldots$

3. Comparison of Photons versus Carbon lons in Single Fraction Therapy of Lung Cancer $\quad 71$

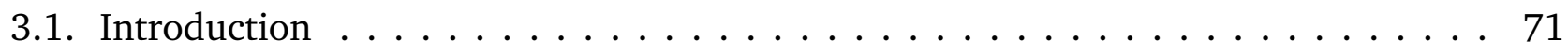

3.2. Materials and methods . . . . . . . . . . . . . . . . . 72

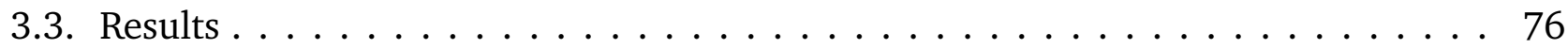

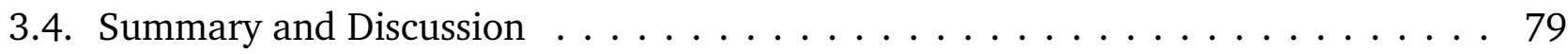

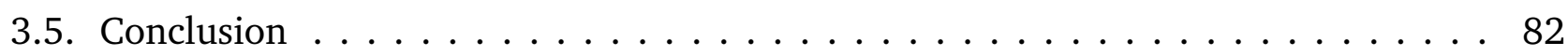

4. Intensity modulated particle therapy for multiple targets 83

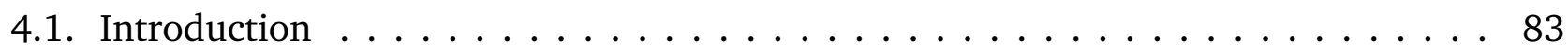

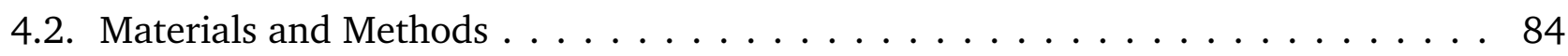

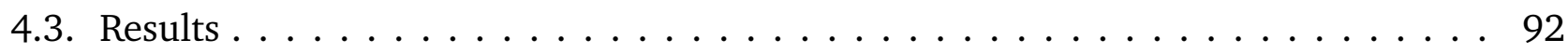

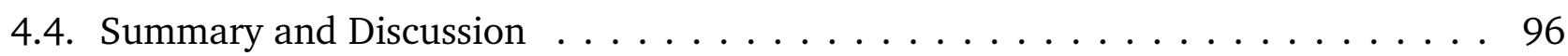

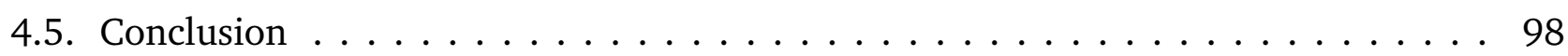

5. Discussion $\quad 99$

5.1. Deformable image registration and validation . . . . . . . . . . . . . 99

5.2. Treating non-small cell lung cancer with particle therapy . . . . . . . . . . . 101

$\begin{array}{lr}\text { 6. Conclusion and Outlook } & 105\end{array}$ 
$\begin{array}{ll}\text { A. Appendix of Chapter } 2 & 107\end{array}$

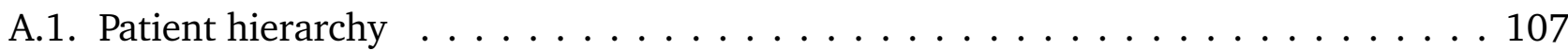

B. Appendix of Chapter $3 \quad 109$

B.1. Organs at risk dose limits . . . . . . . . . . . . . . . . . . . . . 109

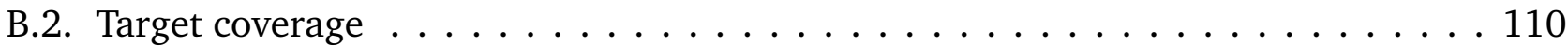

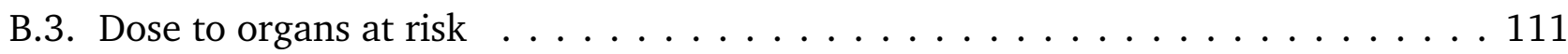

C. Appendix to Chapter $4 \quad 117$

C.1. Target coverage . . . . . . . . . . . . . . . . . . 117

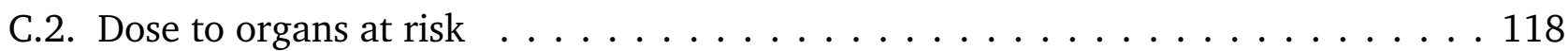

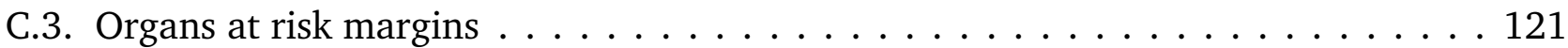




\section{List of Abbreviations}

3DCRT three dimensional conformal radiotherapy

4D-CT time resolved computed tomography

4Dopt time resolved optimization

AP anterior-posterior

CNAO National Centre of Oncological Hadrontherapy, Pavia, Italy

CT computed tomography

CTV clinical target volume

$D_{99 \%} \quad$ minimum dose delivered to $99 \%$ of volume

$D_{\text {Max }} \quad$ maximum dose delivered to a single voxel

$D_{\text {Mean }}$ mean dose delivered to volume

DIR deformable image registration

DIRQA quality assurance of deformable image registration

DNA deoxyribonucleic acid

DSB double strand breaks

DVH dose volume histogram

FWHM full width at half maximum

GSI GSI Helmholtzzentrum für Schwerionenforschung GmbH

GTV gross tumor volume

HIT Heidelberg Ion-Beam Therapy Centre

HU Hounsfield unit

ECG electrocardiography

IES iso-energy slice

IMPT intensity modulated particle therapy

IMRT intensity modulated radiotherapy

ITV internal target volume

LEM local effect model

LET linear energy transfer
LR left-right

MLC multileaf collimators

MRI magnetic resonance imaging

NIRS National Institute of Radiological Sciences, Chiba, Japan

noPM no pacemaker

OAR organ at risk

PET positron emission tomography

PM pacemaker

PMMA polymethyl methacrylate

PSI Paul Scherrer Institut

PTV planning target volume

RBE relative biological effectiveness

RTOG Radiotherapy Oncology Group

SA smaller airways

SI superior-inferior

SBRT stereotactic body radiation therapy

SFUD single field uniform dose

SOBP spread out Bragg peak

SSB single strand break

TRiP treatment planning for carbon ion radiotherapy

$V_{20 \%} \quad$ volume which received at least $20 \%$ of the target dose (dose coverage)

VMAT volumetric modulatec arc therapy 


\section{Motivation}

In 2013 every fourth death in Germany was due to cancer and approximately 45000 deaths were from lung and bronchial cancer [German Federal Statistical Office, 2015]. In the last 30 years, there was a $180 \%$ increase of deaths due to lung and bronchial cancer for women. The standard course of treatment for lung cancer is surgery, chemotherapy, radiotherapy or a combination of these. Surgery is usually the first choice of treatment for early stages of lung cancer. In recent years, however, state of the art photon radiotherapy, called stereotactic body-radiation therapy (SBRT) showed promising results [Baumann et al., 2009, Greco et al., 2011]. The core innovation of SBRT was to deliver high ablative and focused doses in very few fractions, usually $1-5$, compared to up to 30 fractions in conventional radiotherapy. Due to the high fraction doses up to $24 \mathrm{~Gy}$, dose to critical structure must be considered carefully.

In the last twenty years, ion beam therapy has proven to be a promising alternative to photon radiotherapy. Higher tumor control rates and better dose conformity can be achieved with superior physical and biological ion properties, when compared to photons [Tsujii et al., 2008, Durante and Loeffler, 2010]. A recent review made by Kamada et al reported a high 3-year survival rate for carbon-ions (76.9\%) for treating lung cancer in a single fraction, with no late treatment-related complications [Kamada et al., 2016]. The treatment used passive beam scanning, where patient specific absorbers are used to conform the dose to the tumor. Active beam scanning, on the other hand, can provide even better dose shaping, which is essential in hypo-fractionated treatment. However, interaction between tumor and scanned beam motion, called interplay, can severely degrade dose distribution in the breathing patient. Therefore designated motion mitigation techniques must be used for successful treatment of lung cancer with active beam scanning [Bert et al., 2008].

Tumors in the abdomen region (liver and pancreas tumors) with were already successfully treated scanned ion beams at HIT, Heidelberg (Germany) and CNAO, Pavia (Italy) [Habermehl et al., 2013, Rossi, 2016] and first lung cancer patients are being treated at NIRS, Chiba (Japan) [Mori et al., 2016]. Studies on impact of scanned ion beams on lung cancer treatment are thus warranted, so that eligible patients can be identified. This is crucial, as ion therapy is expensive and clinical availability is limited - its application should thus be focused on patients who will benefit the most.

In this thesis, we will address this challenge of treating lung cancer patients with active beam scanning in a direct comparison between SBRT and scanned carbon-ion therapy. Characteristics of patients particularly suited for carbon-ion therapy will be identified for a possible future treatment at designated facilities in Marburg and Heidelberg. 


\section{Scope of this work}

This is the first in silico comparison between SBRT and active scanning carbon-ion for non-small cell lung cancer (NSCLC). Time-resolved (4D) dose distributions will be studied on a large patient dataset, including patients with multiple metastases.

In order to create carbon-ion treatment plans and calculate 4D doses, contours have to be propagated from the planning computed tomography (CT) to all motion states of a 4D-CT. Additionally, motion between 4D-CT states has to be quantified, and doses have to be accumulated on a reference state. This will be achieved with deformable image registration (DIR). DIR is a powerful image processing tool, but is based on large degrees of freedom and consequently associated with a large potential for error. Especially the propagation of dose with DIR is a highly debated issue in current research. Therefore, a designated tool for DIR quality assurance (DIRQA) will be developed. A verification of DIR and DIRQA will be done on available 4D-CT datasets.

To show the potential of scanned carbon-ions in handling NSCLC, treatment plans for 19 patients, which were actually treated with SBRT, will be calculated. Afterwards, static and 4D doses with and without motion mitigation will be analyzed. Doses to targets and organs-at-risk (OAR) will be analyzed and compared between carbon-ions and SBRT.

Patients with advanced stage disease and multiple lesions in the lungs have an exceptionally dismal prognosis. A strategy to apply intensity modulated particle therapy on multiple targets will be developed and implemented in the GSI in-house treatment planning system TRiP98. Different 4D optimization strategies will be tested in a dataset of such patients to cope with target motion in this specific setting. Again, plans will be compared to actually delivered SBRT doses, both with respect to reduction of normal tissue exposure, but also to investigate whether PT can deliver full ablative doses to these patients where SBRT could not due to normal tissue constraints.

The structure of this dissertation is as follows. Chapter 1 will present an overview of physical and biological fundamentals of radiotherapy. Photon and particle radiotherapy will be presented, with an emphasis on the treatment of moving targets. Additionally, a description of lung cancer will be given. Chapter 2 will present tools to handle DIR and DIRQA and verification of these tools. In chapter 3 , comparison between SBRT and carbon-ions will be investigated on lung cancer patients. IMPT for patients with multiple metastases in lung will be investigated in chapter 4. Overall results will be discussed in chapter 5 and the thesis will be concluded in chapter 6. 



\section{Introduction - research background and fundamentals}

\section{Contents}

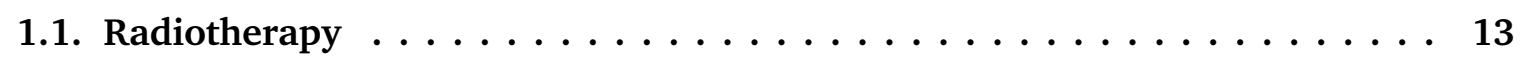

1.2. Physical and biological basics of radiotherapy $\ldots \ldots \ldots \ldots \ldots$

1.2.1. Interaction of radiation with matter . . . . . . . . . . . . . . . 14

1.2.2. Radiobiology . . . . . . . . . . . . . . . . . . . . . . 19

1.2.3. Application technique . . . . . . . . . . . . . . . . 25

1.2.4. Motion in radiotherapy $\ldots \ldots \ldots \ldots \ldots \ldots$

1.2.5. Treatment planning . . . . . . . . . . . . . . . 34

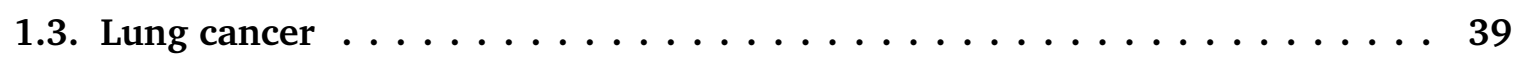

1.3.1. Epidemiology . . . . . . . . . . . . . . . . . . . . 39

1.3.2. Non-small cell lung cancer . . . . . . . . . . . . . . . . . . . . 40

\subsection{Radiotherapy}

Ionizing radiation has been used for treating tumors since the discovery of X-rays in 1895. In the beginning, only superficial diseases were treated, but as time and technology progressed X-ray tubes gained on voltage, allowing treatment of deeper seated tumors.

The radiation from a linear accelerators was first used in medicine in 1953. The cure rates increased tremendously due to the collimated beam and higher energies compared to X-ray tubes. The next big milestone was the introduction of computers in the field of radiotherapy. This led to better diagnostic tools, such as computed tomography scans (CT), magnetic resonance imaging (MRI) and positron emission tomography (PET). The location of the tumor could be estimated better with these tools and hence the treatment was improved. The potential of computers was afterwards exploited also in the treatment planning process, resulting in intensity modulated radiation therapy (IMRT) which, together with diagnostic tools, provides an exact dose shaping in accordance to patient specific tumors.

In 1946, a paper from R. Wilson first described the application of protons for cancer treatment [Wilson, 1946]. It was shown that protons have a preferable depth dose profile compared to 
photons. First patient treatment followed in the early 1950's at Lawrence Berkeley Laboratory, USA. Heavier ions, such as ${ }^{2} \mathrm{He},{ }^{20} \mathrm{Ne}$ and ${ }^{6} \mathrm{C}$ were later also used for treatment. In the beginning only passive beam delivery (see Section 1.2.3) was used for treatment and a active beam solution (see Section 1.2.3) was developed in the 1990's at Paul Scherrer Institute (PSI), Villigen (Switzerland) for protons and at GSI, Darmstadt (Germany) for carbon ions.

\subsection{Physical and biological basics of radiotherapy}

The aim of radiotherapy is to kill tumor cells and prevent further growth, while sparing healthy tissue. Both radiotherapy modalities (photons and ions) use the same principle to eliminate cancer cells - they aim to damage the deoxyribonucleic acid (DNA) through ionization. The actual physical and biological mechanism behind both modalities will be explained in detail in the following section.

\subsubsection{Interaction of radiation with matter}

The interactions between photons with matter and ions with matter are quite different, as can be seen from depth dose distributions in Fig. 1.1. Photons deposit the highest local dose shortly after entering the matter (at the energies used in radiotherapy). Ions, on the other hand, deposit most of their dose right before they stop in the so-called Bragg Peak region. The position of the Bragg Peak depends on the energy of the ions, which is exploited in the treatment of deep seated tumors.

\section{Dose definition}

An important quantity in radiotherapy is dose, $D$, defined as the ratio of the absorbed energy $d E$ per mass element $d m$ [ICRU, 1993b]:

$$
D=\frac{d E}{d m} \quad\left[G y=\frac{J}{k g}\right]
$$

Energy loss in a thin layer of material is described as $d E / d x$. Dose can be then rewritten as:

$$
D=\frac{d E}{d x} \times \frac{1}{F} \times \frac{1}{\rho}
$$

where $F$ is the fluence and $\rho$ the material density. 


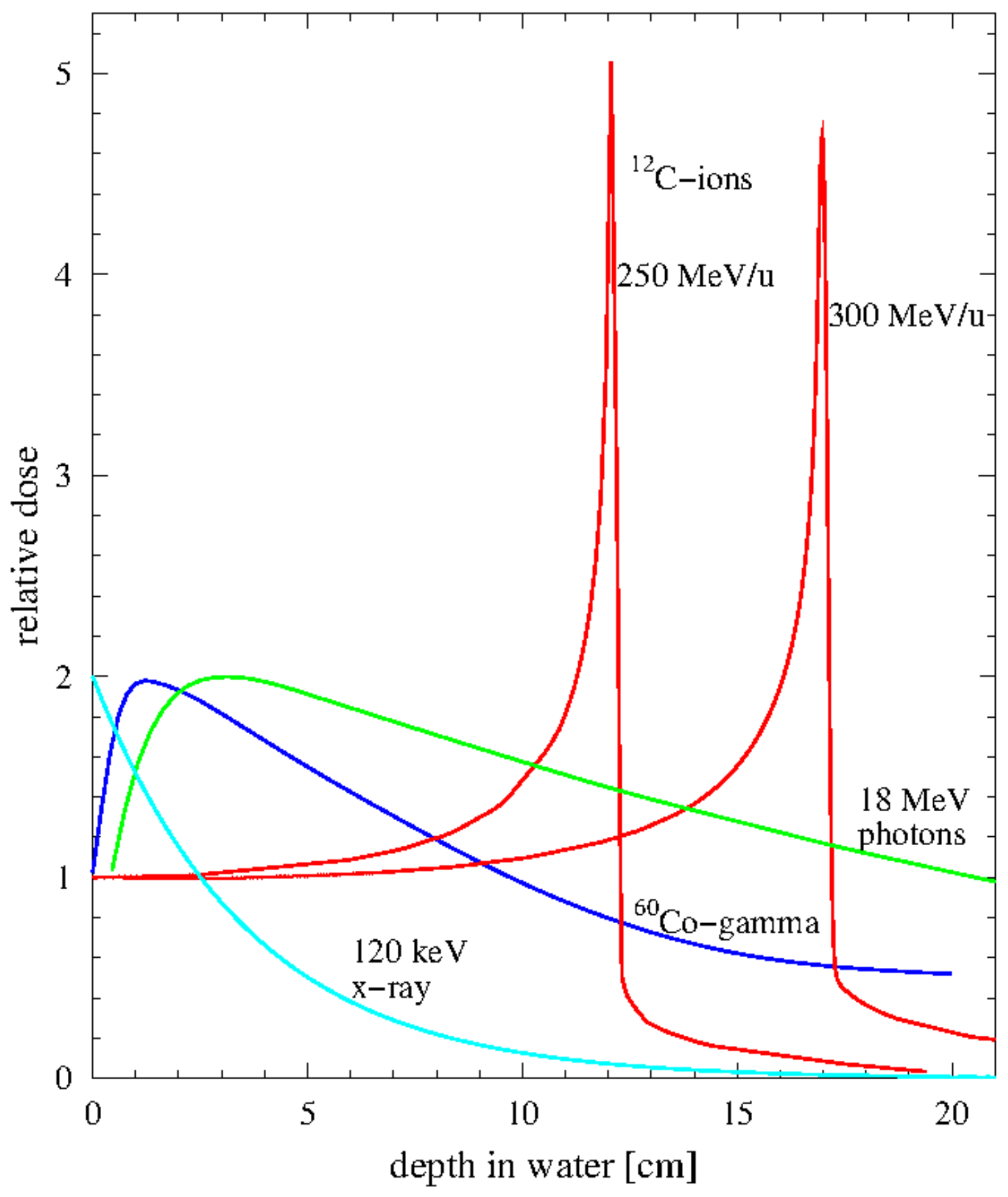

Figure 1.1.: Photon and carbon ions depth dose distributions at different energies. Photons start with a build up, which is then followed by an exponential decrease. Ions deposit most of the dose at the end of the particle track - the Bragg peak. Figure taken from [Schardt et al., 2010] 
Photons mostly interact with matter in one of the following ways: coherent or Rayleigh scattering, photoelectric effect, Compton scattering and pair production. The cross section $\sigma$, for each of these processes depends as well on the energy of the incident photons as on the atomic number of the absorbing material [Lilley, 2006]. The decreasing photon intensity in matter, $I$, can be described as:

$$
I=I_{0} \cdot e^{-N \sigma x}=I_{0} \cdot e^{-\mu x}
$$

where $I_{0}$ stands for the initial intensity of the photons, $x$ the depth of the material in units of length, $N$ the atomic density of the material and $\mu$ the attenuation coefficient. The cross section, $\sigma$ is the sum of all possible Interaction processes:

$$
\sigma=\sigma_{\text {rayleigh }}+\sigma_{\text {photoelectric }}+Z \sigma_{\text {compton }}+\sigma_{\text {pairproduction }}
$$

The energy range of photons used in radiotherapy is between $100 \mathrm{keV}$ and $25 \mathrm{MeV}$. The dominating process in this energy range is Compton scattering [Alpen, 1998]. The electrons resulting from Compton interaction scatter mostly in a forward direction. Therefore a maximum of the depth-dose profile occurs when electrons stop at a certain depth, the mean electron range. After this build up the dose deposition decreases exponentially (see Fig. 1.1 and Equation 1.3).

\section{Interaction of ions with matter}

Ions can interact with matter either with elastic Coulomb scattering from target nuclei (nuclear stopping) or with inelastic collision with target electrons (electronic stopping). At the ion energies used in radiotherapy, which are less then $500 \mathrm{MeV} / \mathrm{u}$, the electronic stopping is the dominating interaction. The result is ionization and excitation of the target atoms.

The mean rate of the ions energy loss in matter is described by the Bethe-Bloch formula [Bethe, 1930, Bloch, 1933]. Since we are interested in low ion energies, a non-relativistic approximation can be made:

$$
-\left\langle\frac{d E}{d x}\right\rangle=\frac{4 \pi N_{e} z_{e f f}^{2}}{m_{e} v^{2}}\left(\frac{e^{2}}{4 \pi \epsilon_{0}}\right)^{2}\left[\ln \left(\frac{2 m_{e} v^{2}}{I}\right)+\text { correction }\right]
$$

here $N_{e}$ is the material's electron density, $e$ and $m_{e}$ are the charge and mass of an electron, $\epsilon_{0}$ the electrical field constant and $I$ the mean excitation energy of the absorber material. Barkas formula [Barkas, 1963] can be used for the approximation of the effective projectile charge $z_{\text {eff }}$ : 


$$
z_{e f f}=z\left(1-e^{-125 \beta z^{\frac{2}{3}}}\right)
$$

where $\beta$ is the projectile speed in units of $c$.

The energy loss of the ions is proportional to $z_{\text {eff }}$ and inversely proportional to $v^{2}$. The shape of the curve in Fig. 1.1 can be explained as following: Ions enter the matter with a high velocity, resulting in a small energy deposition. Their velocity gradually decreases, which in turn increases the energy deposition. The maximum of the energy loss occurs right when the ions stop and it is called Bragg peak.

\section{Lateral scattering and range straggling of ions}

As mentioned ions interact with matter mostly via electronic stopping at energies used in radiotherapy. However, nuclear stopping still occurs and it is the main reason for lateral scattering. The angular spread of ions is dependent on the mass of the target nuclei and on the momentum of the incident ions [Molière, 1948]. The lateral scattering is proportional to the mass of the target nuclei and inversely proportional to the momentum of incident ions. Carbon ions have thus less lateral scattering then protons. Experiments have shown that carbon ions have three times smaller angular spread compared to protons at the same range in water $(15.6 \mathrm{~cm}, 150$ $\mathrm{MeV} / \mathrm{u}$ protons and $285 \mathrm{MeV} / \mathrm{u}^{12} \mathrm{C}$ ions) [Schardt et al., 2010].

Statistical fluctuations of specific electronic stopping events cause range straggling of ions. If the number of collisions is high or the material is thick enough these fluctuations can be approximated by a Gaussian probability distribution [Bohr, 1940, Ahlen, 1980]. The straggling width $\sigma_{R}$ is proportional to:

$$
\sigma_{R} \propto R / \sqrt{M}
$$

where $R$ is the mean range of ions and $M$ the ion mass. Thus, the heavier the ion is, the less range straggling it has. Carbon ions have 3.5 smaller range straggling when compared to protons [Schardt et al., 2010].

\section{Nuclear fragmentation}

When transversing through matter ions (except protons) can be fragmented into ions with lower atomic number. The lower $\mathrm{Z}$ fragments travel in the same direction as projectile ions and have a significant contribution to the deposited dose (see Fig. 1.2). It is thus essential that fragments are included in the treatment planning, so that an accurate dose can be calculated. 
After colliding with the target projectile fragments enter excited state. A de-excitation occurs through emission of nucleons, nucleon clusters and photons. Two of the possible fragments of projectile ${ }^{12} \mathrm{C}$ ions are isotopes ${ }^{11} \mathrm{C}$ and ${ }^{10} \mathrm{C}$, which are both $\beta^{+}$emitters [Kraft, 2000]. The resulting positron is annihilated with electrons in matter, creating two photons, which travel in opposite direction. PET (Positron Emission Tomography) can take advantage of the process without exposing patient to additional radiation.

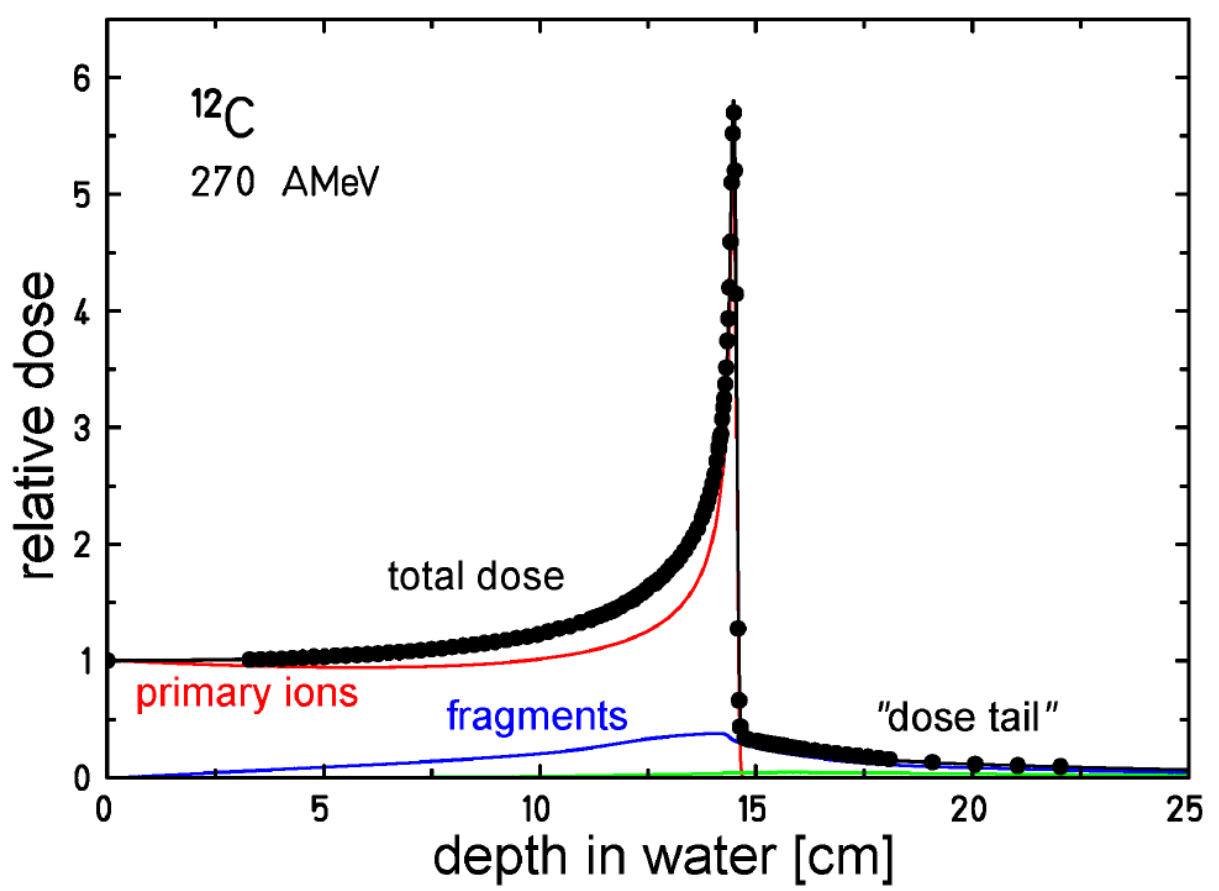

Figure 1.2.: Impact of fragmentation on a depth dose distribution of carbon ions. Main contribution to the overall deposited dose (black line) comes from the primary ions (red line). The produced fragments (blue line) have a smaller, but non-negligible, impact. The most profound effect is seen in the dose tail behind the Bragg peak, where the dose comes only from fragments. Figure taken from [Groezinger, 2004]

\section{Secondary electrons and track structure}

Ions used in radiotherapy loose most of their energy via inelastic Coulomb scattering with target electrons. Electrons liberated from the target by ions are called secondary electrons or $\delta$-electrons. $\delta$-electrons travel through matter, scatter further and may produce secondary ionizations of the target atoms. When $\delta$-electrons energies are larger than $>50 \mathrm{eV}$, ionization becomes the dominant process, which produces a large number of additional electrons [Kraft, 2000, Schardt et al., 2010].

The radial dose profile and track diameter is defined by the energy spectrum of the $\delta$ electrons. Most of the $\delta$-electrons are concentrated around the projectile ions path, since they receive small energy transfers or are scattered in the direction of incident ions. Differ- 
ent models [Chatterjee and Schaefer, 1976, Katz and Cucinotta, 1999] and Monte Carlo simulations [Paretzke, 1986, Krämer, 1995] predict radial dose fall-off approximately with $1 / r^{2}$ for radial distance $r$. Varma et. al. have confirmed this experimentally [Varma et al., 1977]. The maximum radial distance $r_{\max }$ is defined by the most energetic $\delta$-electrons, which are related to energy, E, of the projectile ions [Kiefer and Straaten, 1986].

$$
r_{\max }=E^{1.7}
$$

Following equation $1.5, E$ is correlated to $Z^{2}$ and $1 / \beta^{2}$, meaning that track structure is highly dependent on the projectile ion species and the energy as demonstrated in Fig. 1.3: Carbon ions have a denser ionization structure compared to protons [Krämer and Durante, 2010]. $\delta$ electrons have low energies, and thus the $r$ is on nanometer scale. As the energy of projectile ions decreases, their stopping power increases and causes significantly larger number of $\delta$ electrons. The energy deposited by $\delta$-electrons in medium is described using the linear energy transfer (LET), which is closely related to $d E / d x$. Fast ions with low ionization have a small LET, while slow ions with a large ionization have a high LET.

\subsubsection{Radiobiology}

Ionizing radiation (photons and ions) causes damage throughout the cell. However the most susceptible part to radiation is the carrier of genetic information, the DNA, located in the cell nucleus [Munro, 1970]. Radiation can damage DNA directly or indirectly.

Ionization and consequent destruction of DNA molecular bonds via radiation is a direct effect (see Fig. 1.4b) and is typical for high-LET radiation. On the other hand, an indirect effect is when radiation hydrolysis water around DNA and produce highly reactive hydroxyl-radicals, $\mathrm{OH}$ (see Fig. 1.4a). Even though $\mathrm{OH}$ radicals decay fast, they are still able to cause severe damage to DNA. The formation of $\mathrm{OH}$ is typical for low-LET radiation like photons. The two processes, direct and indirect, are not exclusive and can damage DNA in parallel. 


\section{Protons in $\mathrm{H}_{2} \mathrm{O}$ Carbon Ions in $\mathrm{H}_{2} \mathrm{O}$}

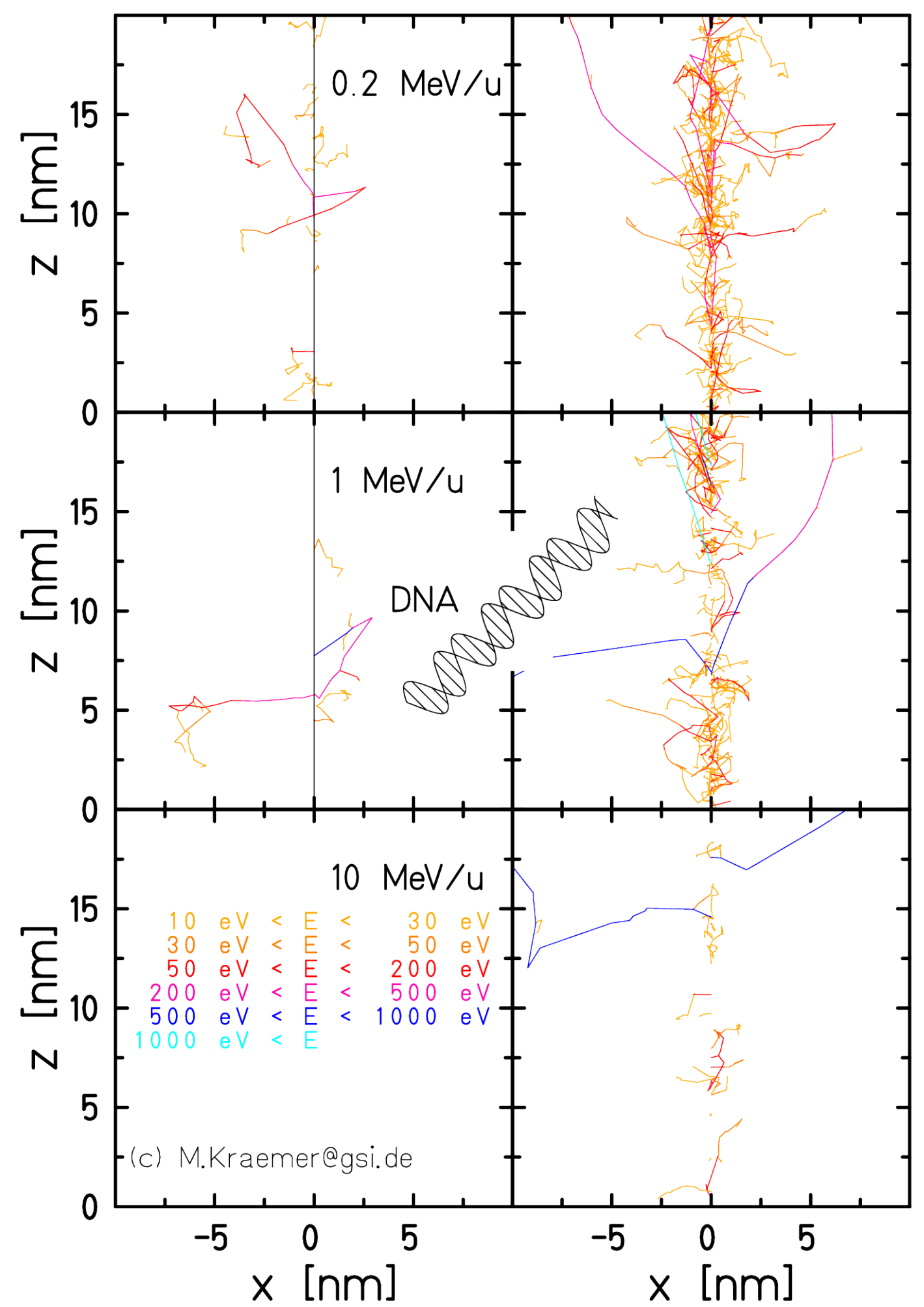

Figure 1.3.: Track structure of ions in water at different energies. The distribution of $\delta$-electrons is highly dependent on ion species and their energy. A molecule of DNA is displayed for size comparison. Figure courtesy of Michael Krämer. 


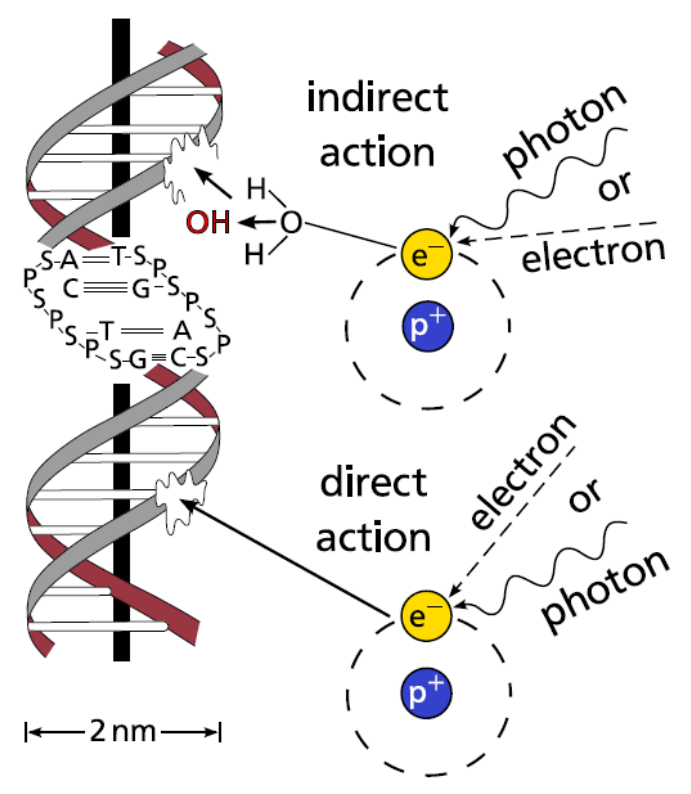

(a)

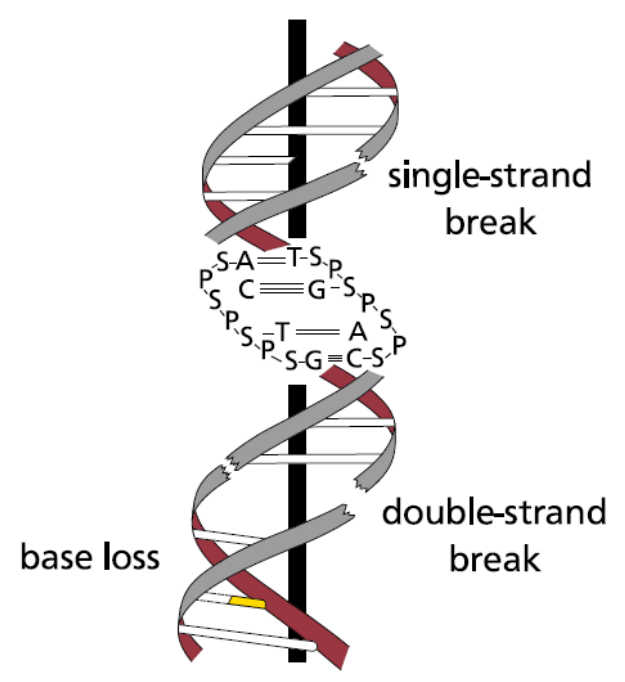

(b)

Figure 1.4.: Types of DNA damage caused by radiation (a) Indirect damage occurs, when radiation forms free radicals hydroxyl radicals $(\mathrm{OH})$, which can damage DNA. (b) Direct effects of radiation can cause single or double-strand breaks. Figure taken from [Richter, 2012]

Damage to DNA can result in either single strand breaks (SSB) or double strand breaks (DSB) as shown in Fig. 1.4b). When one of the double strands in the DNA helix is destroyed (SSB), it can usually be easily repaired by cell repair-mechanisms, since the complementary base is intact. If both strands are destroyed (DSB) the DNA damage is much more complex and leads to the breakage of the chromatin. The cell repair-mechanisms can handle DSB as well, albeit not as efficient as SSB. However if there are clustered DSBs, the damage is usually too severe for repair-mechanisms to undo it. The changes in damaged DNA can lead to carcinogenesis or cell death. The aim of radiotherapy is to cause an apoptosis - a controlled self-inactivation of the cell triggered by the DNA damage. Beside apoptosis, cell can also undergo necrosis, an uncontrolled cell death. Cell necrosis often causes response from the immune system, leading to inflammation, which radiotherapy strives to avoid. DNA can also be damaged to such extent, that cell cannot proliferate indefinitely - a effect known as clonogenic cell death.

\section{Relative Biological Effectiveness}

Fig. 1.3 shows the size of a DNA molecule in comparison with proton and carbon ion distribution of $\delta$-electrons around their track. A clustered DSB occurs preferably around the Bragg peak due to large ionization densities. Less cells will survive a clustered DSB, compared to DSB or 
SSB. Ions have large ionization density, which is one of the main advantages over photons in radiotherapy. Since most of the clinical experience about cell response to radiation comes from photons, the biological effect of ions is usually described relative to a reference photon response. Relative biological effectiveness (RBE) is therefore defined as the ratio of the reference photon dose to the dose level of a specific ion radiation at the same biological effect (isoeffect):

$$
R B E=\left.\frac{D_{\text {photon }}^{\text {ref }}}{D_{\text {ion }}}\right|_{\text {isoeffect }}
$$

It is important to note at this point that RBE values are valid only for the same effect - the same biological endpoint and the same reference radiation. The most interesting biological endpoints in radiotherapy are cell survival and side effects. RBE values are usually obtained from cell survival curves (see Fig. 1.5). Cell survival, S, is commonly modeled by an exponential linear-quadratic (LQ) model [Fowler, 1989]:

$$
S(D)=e^{-\alpha D-\beta D^{2}}
$$

$\alpha$ is a coefficient related to a single event cell killing and $\beta$ coefficient related to a double event cell killing. The ratio of $\alpha / \beta$ is a characteristic of the cell type, namely the tissue capacity to repair radiation damage. A small $\alpha / \beta$ ratio means a cell is radioresistive (high repair capacity) and vice versa. As seen in Fig. 1.5 and Eq. 1.10, RBE values are dependent on the dose level. Hence in ion radiotherapy, beside the physical absorbed dose, a photo-equivalent or biological dose incorporating the RBE also plays an important role. The unit for biological dose is Gy (RBE) [ICRU, 2007].

Besides the dose level, RBE also depends on the LET, the particle species and the tissue type [Kraft, 2000]. Therefore RBE modeling is a complex topic. At GSI, RBE is calculated using the local effect model (LEM) developed by Scholz et al. [Scholz and Kraft, 1994]. There are two main assumptions in the LEM model. The first is that localized biological effects are independent on the radiation type. The second assumption is that the photon response is the same for all dose levels (high and low). The difference between different radiation types comes from the dose deposition in a small volume in the cell nucleus. At the same total dose, many photons create a homogeneous dose distribution over a cell nucleus, while few ions cause a highly localized dose distribution around their track. 


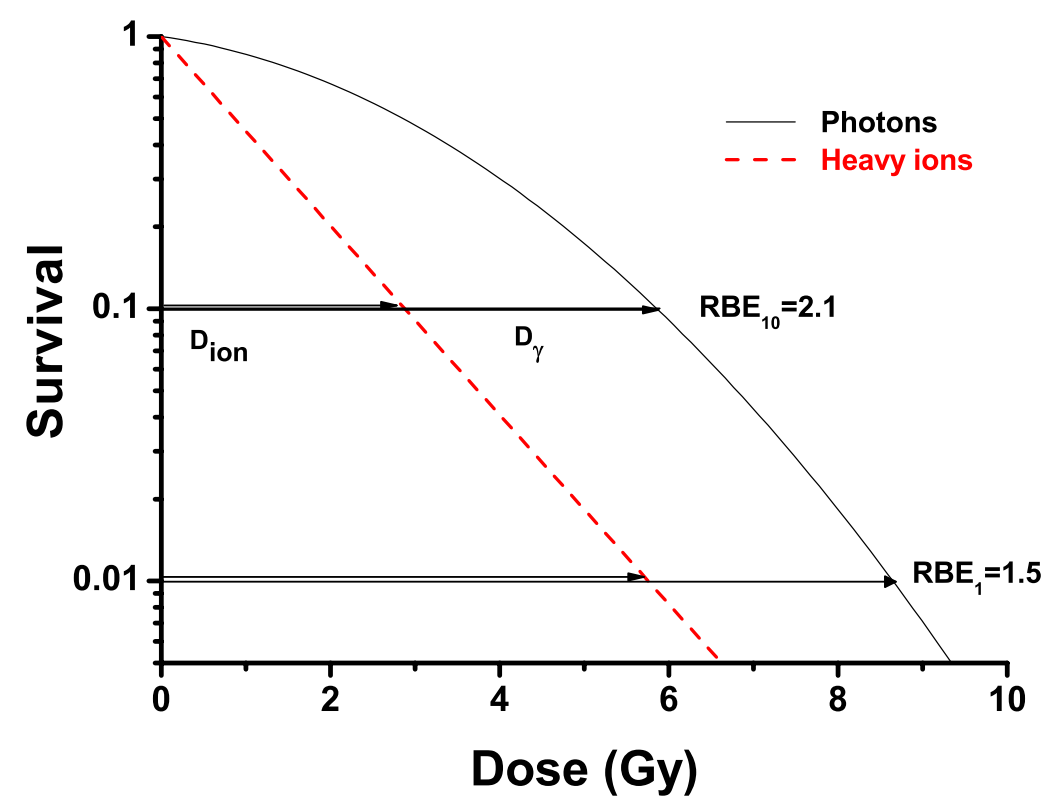

Figure 1.5.: Typical cell survival curve for photons (black solid line) and heavy ions (red dashed line). Photon line shows a typical shouldered form, described by the linear-quadratic model. Heavy ions show a much steeper decrease with dose. The RBE value can be calculated by looking at the dose values at the same survival value - corresponding to the same biological effect. Figure taken from [Schardt et al., 2010]

LEM can thus predict dose response, by comparing photon response at the high local dose level. LEM was used in the GSI pilot project from 1998 - 2007 [Krämer et al., 2000, Krämer and Scholz, 2000] as well as clinically in HIT since 2009. LEM has received several revisions [Elsaesser and Scholz, 2006, Elsaesser and Scholz, 2007, Elsaesser et al., 2009] and experimental verifications [Mitaroff et al., 1998, Krämer and Scholz, 2000, Krämer et al., 2003].

RBE for carbon ions ranges from 1 in the entrance channel, to values around 5 at the Bragg peak [Kraft, 2000]. The highest RBE for carbon ions is right around the Bragg peak, which gives carbon ions a great advantage, since there is an increased biological effectiveness at the target tissue compared to the normal tissue in the entrance channel. In proton therapy a constant RBE value of 1.1 across the treatment field is used [Paganetti et al., 2002].

\section{Fractionation}

Radiotherapy applies a basic principle of radiobiology that dose fractionation spares all cell types. For a given total dose more cells will survive with dose delivered across multiple fractions, compared to a single dose, because cells will have time to repair radiation induced sub-lethal damage between fractions. With a dose $d$ delivered over $n$ fractions, equation 1.10 can be rewritten as [Shrieve and Loeffler, 2011] 


$$
S=\left(e^{-\alpha d-\beta d^{2}}\right)^{n}
$$

The biological effective dose (BED) is defined as:

$$
B E D\left(G y_{\alpha / \beta}\right)=n d\left[1+\frac{d}{\alpha / \beta}\right]
$$

with the total dose $D$ equal to $n \times d$, we can define the fractionation factor $F$ as

$$
F=\left[1+\frac{d}{\alpha / \beta}\right]
$$

so that $\mathrm{BED}=D \times F$. F increases with $d$, but decreases with $\alpha / \beta$. Lower $\alpha / \beta$ (late-responding tissue) means higher $F$ and a higher $\alpha / \beta$ (early responding tissue) moves $F$ towards 1 . As normal tissue typically has a lower $\mathrm{a} / \mathrm{b}$ (around 2) than tumor tissue (around 4-10), fractionation effectively increases the differential dose to the tumor, and thus increases the therapeutic window.

\section{Hypofractionation}

Due to the improvement in radiotherapy, there is a trend to increase the dose per fraction and reduce the number of fractions, called hypofractionation [Lo et al., 2010]. Hypofractionation consists of 1-3 fractions of high doses, up to $24 \mathrm{~Gy}$ in a single fraction (single-dose). It has shown promising results over a wide range of tumors [Yamada et al., 2008, Greco et al., 2011, Halasz and Rockhill, 2013]. The high dose damages the vascular system, which supplies cancer tissue with oxygen and nutrients. The damage to the vascular system and may be the dominant process in the tumor suppression [Fuks and Kolesnick, 2005]. This was originally showed in genetically modified mice, where vascular damage was shown for doses higher than 10 Gy per fraction [Garcia-Barros et al., 2003]. Effect of high doses on vascular system and consequential tumor control was later confirmed in spinal SBRT for doses between 18-24 Gy [Yamada et al., 2008].

While the effect of high doses on the vascular component is apparent, a strong effect of hypofractionation can also be explained with a high BED resulting from the LQ model, as shown in Table 1.1. The radiation to normal tissue limited the use of hypofractionation in the past. Most recent photon therapy, SBRT, can significantly reduce the normal tissue radiation and hence increase the dose per fraction. The scientific community has not yet reached consensus on which mechanism is actually at work behind hypofractionation [Park et al., 2012a]. 
In addition to high tumor control rates, hypofractionation is also beneficial from economical point of view. If the number of fractions is reduced, i.e. from 30 to 1-3, the treatment time and hence the cost is tremendously reduced, which is especially important in PT.

Table 1.1.: BED for different fractionation schemes for two $\alpha / \beta$ ratios. Single fraction (1 $\times 24$ $\mathrm{Gy}$ ) has more than two times higher BED compared to conventional ( $30 \times 2 \mathrm{~Gy}$ ) or 3 x 9 Gy fractionation scheme for $\alpha / \beta=2$.

\begin{tabular}{|l|c|c|c|c|}
\hline \hline & \multicolumn{4}{|c|}{ Fractionation scheme } \\
\hline & $1 \times 24 \mathrm{~Gy}$ & $3 \times 9 \mathrm{~Gy}$ & $5 \times 7 \mathrm{~Gy}$ & $30 \times 2 \mathrm{~Gy}$ \\
\hline BED $\left(\mathrm{Gy}_{\alpha / \beta=6}\right)$ & 120 & 68 & 76 & 80 \\
$\operatorname{BED}\left(\mathrm{Gy}_{\alpha / \beta=2}\right)$ & 312 & 149 & 158 & 120 \\
\hline \hline
\end{tabular}

\subsubsection{Application technique}

The use of X-rays for treating patients has more than a century long history. There is a lot of research and practical knowledge regarding the clinical usage of X-rays. Particle therapy, on the other hand, is a more novel technique, with more patients being treated every year. In the following sections an overview will be given of how the irradiation is actually delivered to the patient for both modalities with the emphasis on ion therapy.

\section{Photon therapy}

In photon therapy high energy x-rays (MV) are used for tumor irradiation. X-rays are produced in a linear accelerator (LINAC). Electrons are accelerated with energies from 2-25 MeV and collided with a high-density target (tungsten), where x-rays are produced via bremsstrahlung. The beam is then directed to the patient and conformed to the tumor shape. The beam is shaped either by blocks at the head of the machine or by a multileaf collimator. A multileaf collimator is made of individual leaves, that can be moved to represent the tumor's shape in the beam's eye view, see Fig. 1.6.

Linear accelerators are usually placed on a gantry, which can be rotated around the patient allowing irradiation from any angle. The arbitrary choice of the beam angle is used in a 3dimensional conformal radiotherapy, where a variable number of beams is used. Each beam is then shaped with a multileaf collimator. An even more precise technique is intensity-modulated radiotherapy (IMRT). IMRT allows treating complex tumor shapes, e.g. when the tumor is in proximity of a critical structure. Volumetric modulated arc therapy (VMAT) uses continuous irradiation together with continuous gantry rotation and multileaf collimator shaping. VMAT is able to produce even more conformal dose shapes than IMRT. 


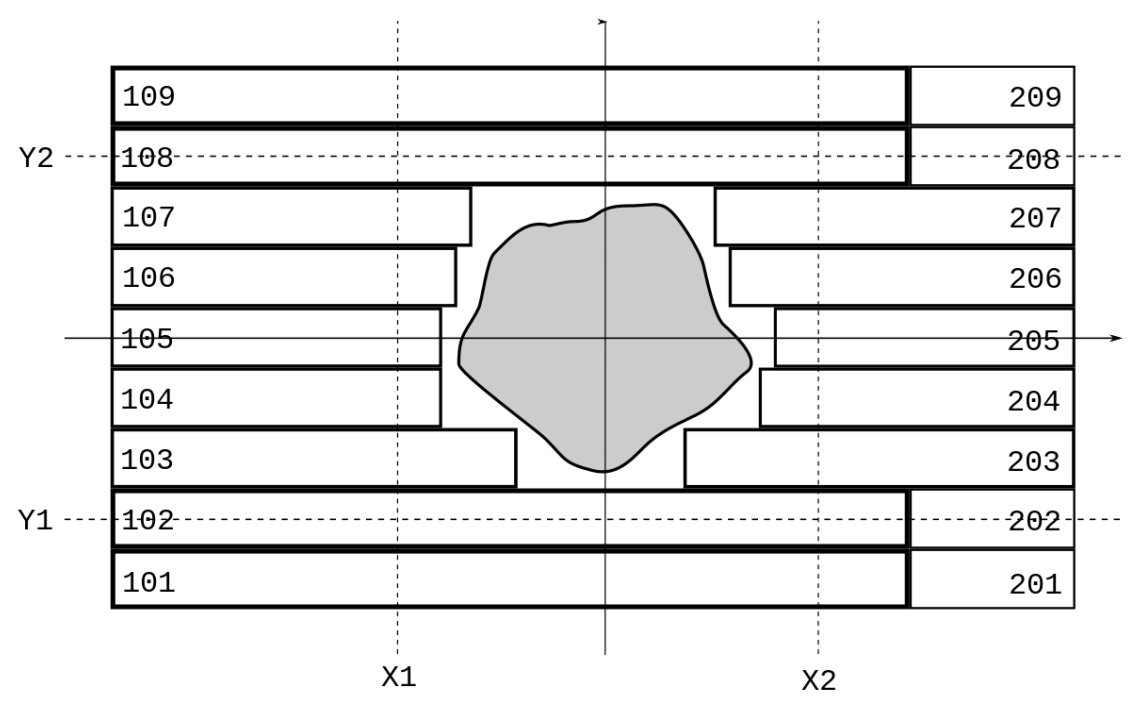

Figure 1.6.: A schematic picture of a multileaf collimator. Individual leaves (marked with numbers) are positioned so that a exit beam from a linear accelerator conforms to the patient tumor in a beam's eye view. Picture taken from [Wikipedia, 2016].

\section{Ion therapy}

In order for ion therapy be successful, ions must be accelerated to appropriate energies (several hundred $\mathrm{MeV} / \mathrm{u}$ ), the beam must be transported to the target area and guided onto the target with the required accuracy.

Ions are accelerated either with cyclotron or synchrotron accelerators. Cyclotrons can be built in a compact design and offer a continuous beam with stable intensities. Cyclotrons are only used for protons. Particle energies can not be modulated and therefore passive energy degraders are needed. Active energy variation is possible with synchrotrons, where a linear accelerator is used to inject ions into the synchrotron and then the beam is regulated with ion optics. Synchrotrons are used in all heavy ion therapy centers, while cyclotrons are most commonly used for proton therapy.

Each tumor has a unique shape, size and position in the patient. Therefore a single Bragg peak would not provide adequate dose and a beam has to be properly shaped. Two beam shaping systems are in use - passive and active beam shaping. In the next two sections both will be explained. 


\section{Passive beam shaping}

The general idea of passive beam shaping is to transform a beam of a fixed single energy into the shape of the tumor. This is done in several steps as schematically shown in Fig. 1.7. Firstly, the beam is broadened using a scattering device (passive double scattering systems or magnetic wobbler) in order to obtain a broad, flat profile. In the next step, the beam is spread out over the required energy range with a range modulator. Usually a range modulator consist of rotating wheels of various thicknesses or a ridge filter [Chu et al., 1993]. A beam of fixed energy is thus expanded into a so-called spread-out Bragg peak (SOBP), which is moved to the required depth using a range shifter. The final two devices in the beam's path are built for each patient individually. A collimator shapes the beam in a lateral direction, while a compensator adjusts the SOBP to the distal edge of the tumor. However, a compensator cannot adjust the dose in the proximal ledge of the tumor, resulting in an access dose to the healthy tissue (hatched area in Fig. 1.7).

Passive beam shaping offers a more robust and faster treatment delivery in contrast to active beam shaping. However, it lacks tumor conformity, the dose cannot be modulated and each patient needs individually tailored devices for each beam used in the treatment. Furthermore, the beam travels through some material, exposing the patient to additional dose due to fragmentation.

\section{Active beam shaping}

In contrast to passive beam shaping, active beam shaping works by dividing the tumor into small points, which are then irradiated using a thin pencil beam. The tumor is first segmented into iso-energy slices (IES) and each of IES is covered with a 2 dimensional grid (raster points). A thin pencil beam is deflected from raster to raster point, irradiating each one with designated dose. The technique allows irradiation of arbitrary shape, without introducing any additional patient specific hardware. The lack of additional material in front of the patient also means less dose due to lesser neutron flux. Furthermore, the dose modulation in each point allows a highly conformal dose distribution with less dose deposited to the healthy tissue. 


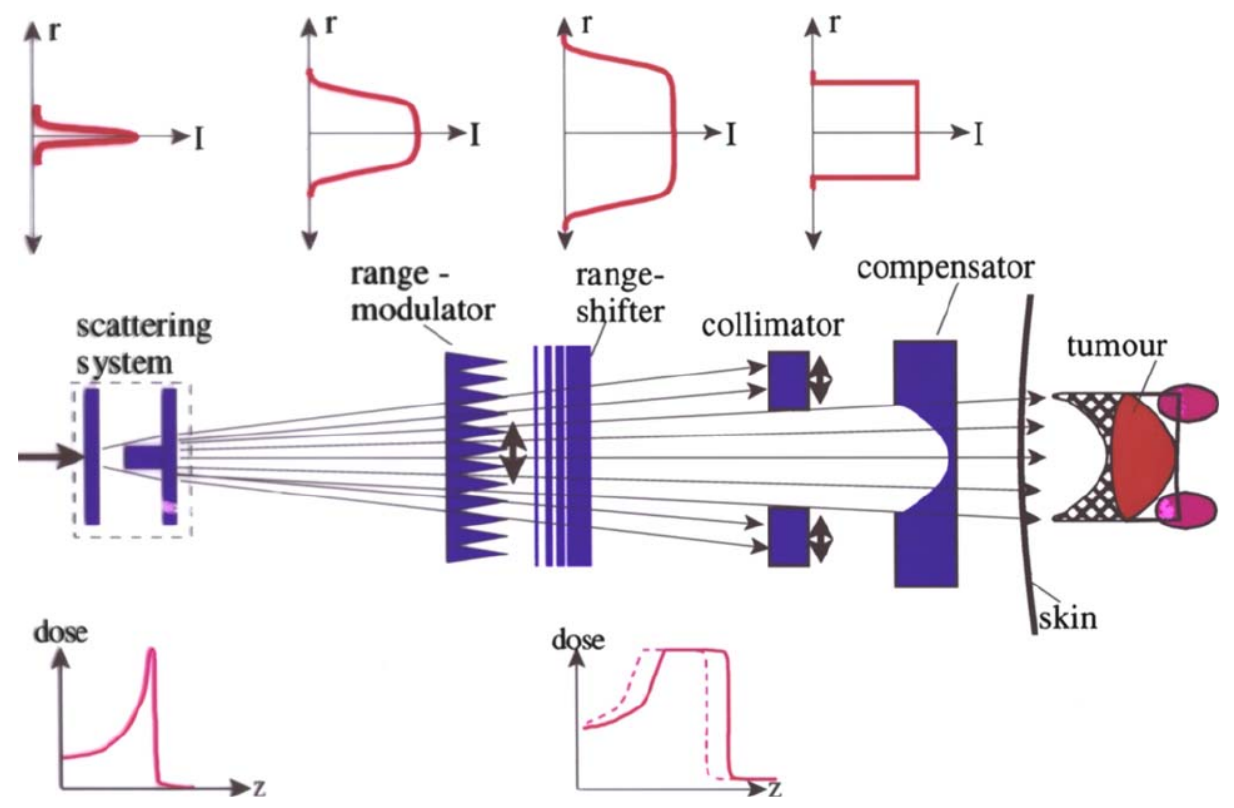

Figure 1.7.: Schematic presentation of a passive beam shaping. A scattering system is used to broaden the beam. Afterwards a range modulator spreads out Bragg Peak to the required energy range. The spread out the Bragg peak is then shifted to a specific energy with a range modulator. Finally, a patient specific collimator and conformator serve for lateral and longitudinal conformity, respectively. The proximal edge of the tumor cannot be shaped, as shown with the hatched area. Figure taken from [Schardt et al., 2010].

There are differences in specifics of active beam shaping. The GSI system of the three-dimensional scanning system will be given here [Haberer et al., 1993, Kraft, 2000, Schardt et al., 2010] and a schematic presentation is shown in Fig. 1.8 and Fig. 1.9. A synchrotron provides a thin pencil beam of ${ }^{12} \mathrm{C}$ ions with a variable energy in the range of 30 $400 \mathrm{MeV} / \mathrm{u}$. The energy defines the position of the Bragg peak in depth. Fig. 1.9b shows how the Bragg peaks are stacked in depth to cover the longitudinal extension of the tumor. The thin pencil beam is guided by two magnetic deflection units to irradiate each raster point. The specific dose in each raster point is calculated in the treatment planing. During treatment the beam stays on each raster point until the intensity monitoring system measures the designated dose. Then it is moved to the next raster point. When the whole IES is irradiated the beam extraction is aborted and the accelerator delivers the next energy for the following IES.

Fig. 1.9a displays how the dose homogeneity in the target is achieved. To achieve flat dose distribution with a Gaussian beam profile, the beam's full width half maximum is three times the lateral raster spacing. Such configuration offers robustness for uncertainties of the beam spots. The spacing between individual IES is usually $3 \mathrm{~mm}$, providing enough overlap between individual Bragg peaks. However, the number of IES should be kept low, since the changing of the beam energy takes most of the time and hence prolongs the treatment. Instead of using 
high number of IES, Bragg peaks are broadened in longitudinal direction by a using ripple filter (RiFi) - a device similar to ridge filters used in passive beam shaping.

polefaces

of dipole magnets

tumor
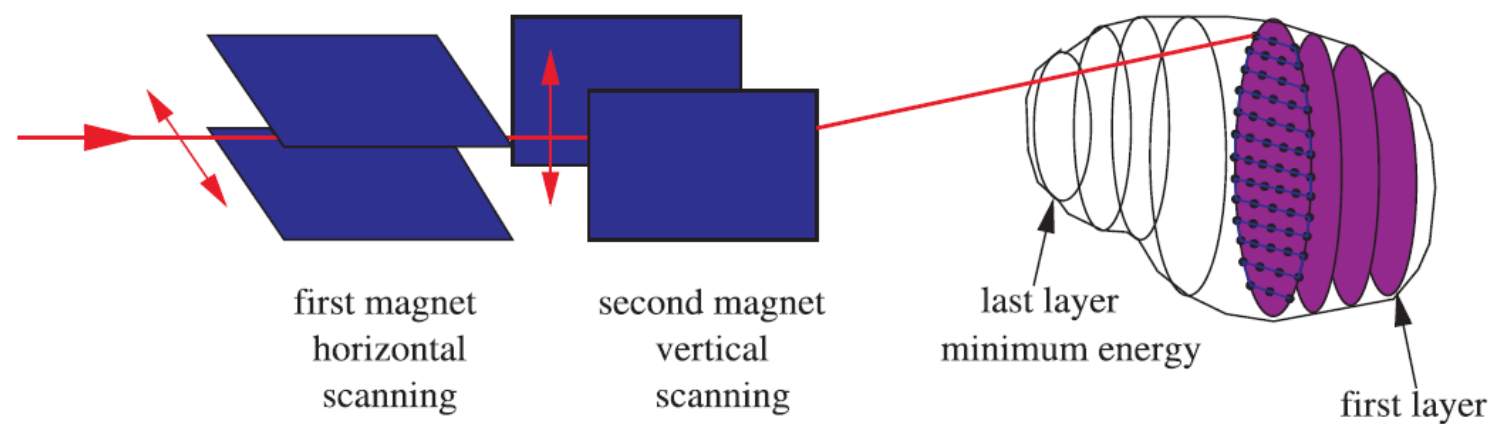

maximum energy

Figure 1.8.: Schematics of GSI's active beam shaping. The tumor is divided into isoenergy slices, which are further overlayed with a 2 dimensional grid. Longitudinal direction (in beam's eye view) is varied with the particle energy from the accelerator, while the lateral is changed via a magnetic scanning system. Figure taken from [Krämer, 2009].

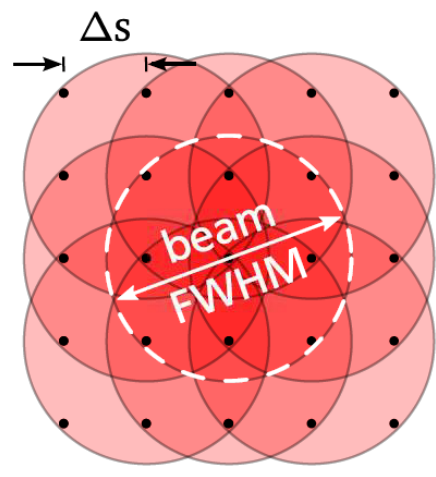

$\mathrm{FWHM}=3 \times \Delta \mathrm{s}$

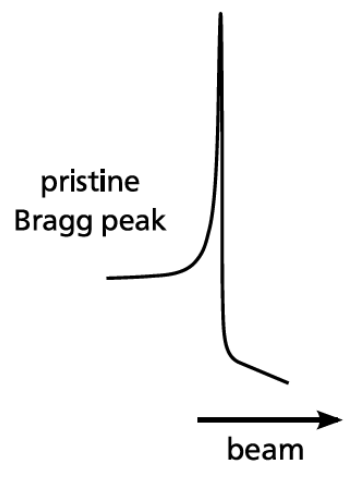

(a)

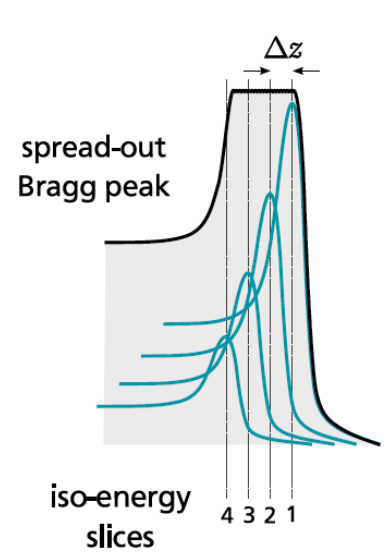

(b)

Figure 1.9.: Schematic presentation of how the target dose homogeneity is achieved in active beam shaping. a) To provide sufficient homogeneity in lateral direction (in beam's eye view) the full width half maximum of beam is three times the spacing between the raster points. b) The Bragg peaks are broadened in depth with a ripple filter and then stacked to provide longitudinal dose homogeneity. Figure taken from [Richter, 2012]. 


\subsubsection{Motion in radiotherapy}

The patient motion can have a profound effect on the radiotherapy. It can cause large deviations from the planned dose, resulting in under- or over-dosage in target and excess dose in OAR. The motion type, its extent and origin is therefore a vast topic of research. A brief introduction will be given here, for an in-depth explanation the reader is pointed to the review by Langen and Jones [Langen and Jones, 2001].

\section{Motion types}

There are three main types of motion: patient positioning, inter- and intra-fractional motion. All three motion types are shown in Fig. 1.10.

Patient position varies between image acquisition (e.g. CT) used for treatment planning and actual delivery. The patient motion introduces changes in tumor shape and tumor position. To overcome patient position uncertainties, patient immobilization and dedicated protocols are used.

Interfractional motion happens between two treatment sessions (fractions) and results in anatomical changes in a patient. It occurs on a time scale of hours and days. For lung cancer patients, the tumor shrinks and the lung density can change between fractions [Mori et al., 2009]. Also changes in breathing pattern can impact treatment delivery. Additionally, the tumor baseline drifts significantly [Sonke et al., 2008]. Repeated imaging and replanning reduces the impact of the interfractional motion, but requires additional time.

Intrafractional motion is mainly caused by respiration and heart beat, but also peristalsis. The time scale ranges from seconds to minutes. In this thesis we investigated the treatment of lung cancer, focusing on respiratory motion. Respiratory motion varies from patient to patient and is responsible for tumor motion from a mm range to a couple of $\mathrm{cm}$ [Shirato et al., 2004]. The tumor size and T-staging are also correlated to tumor motion [Liu et al., 2007]. The respiratoryinduced motion is largest in superior-inferior (SI) direction rather than in the anteriorposterior (AP) or left-right (LR) directions [Seppenwoolde et al., 2002, Britton et al., 2007, Liu et al., 2007]. 

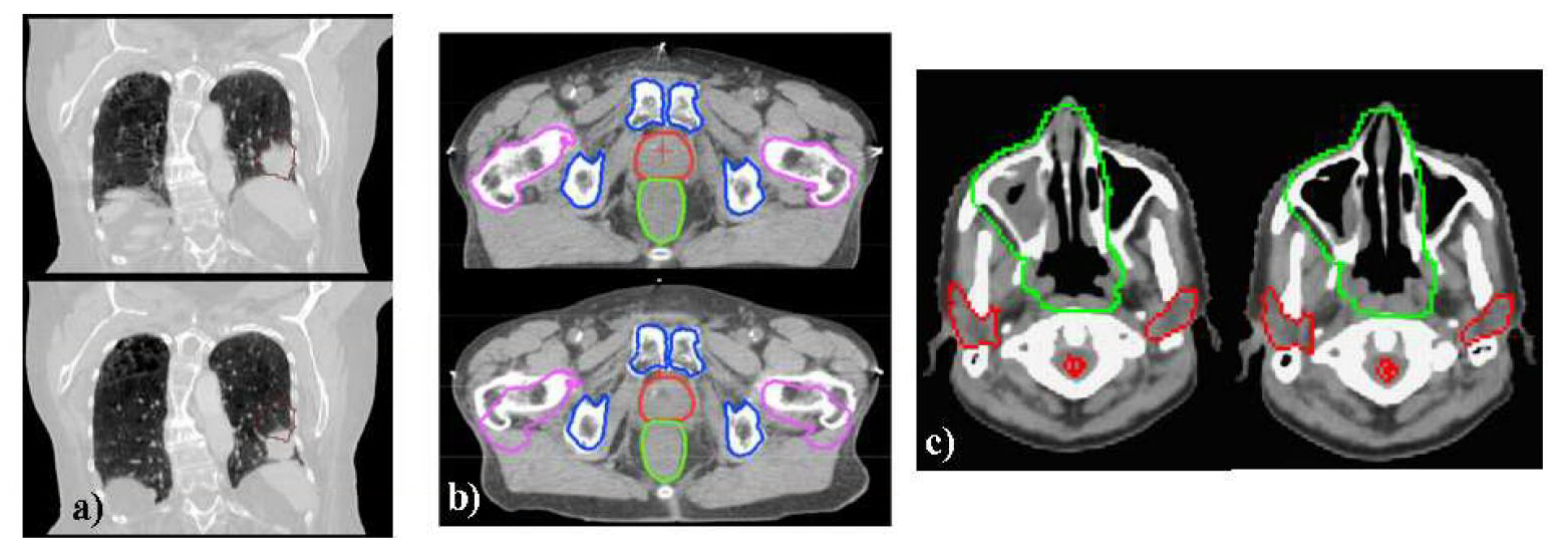

Figure 1.10.: Examples of the three major motion categories. On the left side (a) a lung tumor is displayed, which moves due to the respiration of the patient (intrafractional motion). Interfractional position changes are exemplary shown in the middle (b), where two CT scans of a prostate patient are compared. Density variations between two CT scans are shown in (c). Figure taken from [Engelsman and Bert, 2011]

\section{Motion mitigation techniques}

While all three motion types have to be addressed in treatment planning, special focus will be given on intrafractional motion mitigation. Photon radiotherapy or particle radiotherapy with passive beam shaping use larger safety margins to encompass the whole tumor motion as explained in Section 1.2.5. However, larger safety margins are not enough to mitigate motion when active beam shaping is used. The beam delivery sequence and the target motion interfere with one another, resulting in over- and underdosages in patients. This effect is called interplay and it has been thoroughly reviewed elsewhere [Phillips et al., 1992, Bert et al., 2008]. The effect of interplay depends on many factors, such as motion amplitude, beam direction, starting breathing phase etc. Three main techniques are currently established to counteract interplay: rescanning, gating and beam tracking. Several others techniques exist to reduce the effect of tumor motion, such as abdominal compression, jet ventilation, apneic oxygenation etc., but will not be described here, since the scope of this thesis is on free-breathing patients.

Rescanning is a technique that uses statistical averaging of different interplay patterns [Phillips et al., 1992]. Instead of applying the whole dose $D$ at once, the target is scanned $N$ times, each time irradiated with $\mathrm{D} / \mathrm{N}$. The result is a Gaussian dose distribution around $D$ with no interplay (static case), as shown in Fig. 1.11. With more rescans (larger $N$ ), better dose homogeneity is achieved, because the variance is proportional to $1 / \mathrm{N}$. Technically the method is the easiest to implement of the three mentioned, since no real-time motion monitoring is necessary. The treated volume must be enlarged to at least encompass the target in all motions states (in contrast to gating), which introduces additional dosage to normal tissue. Rescanning 
is currently used at NIRS, Tokyo (Japan) and at some proton centers.

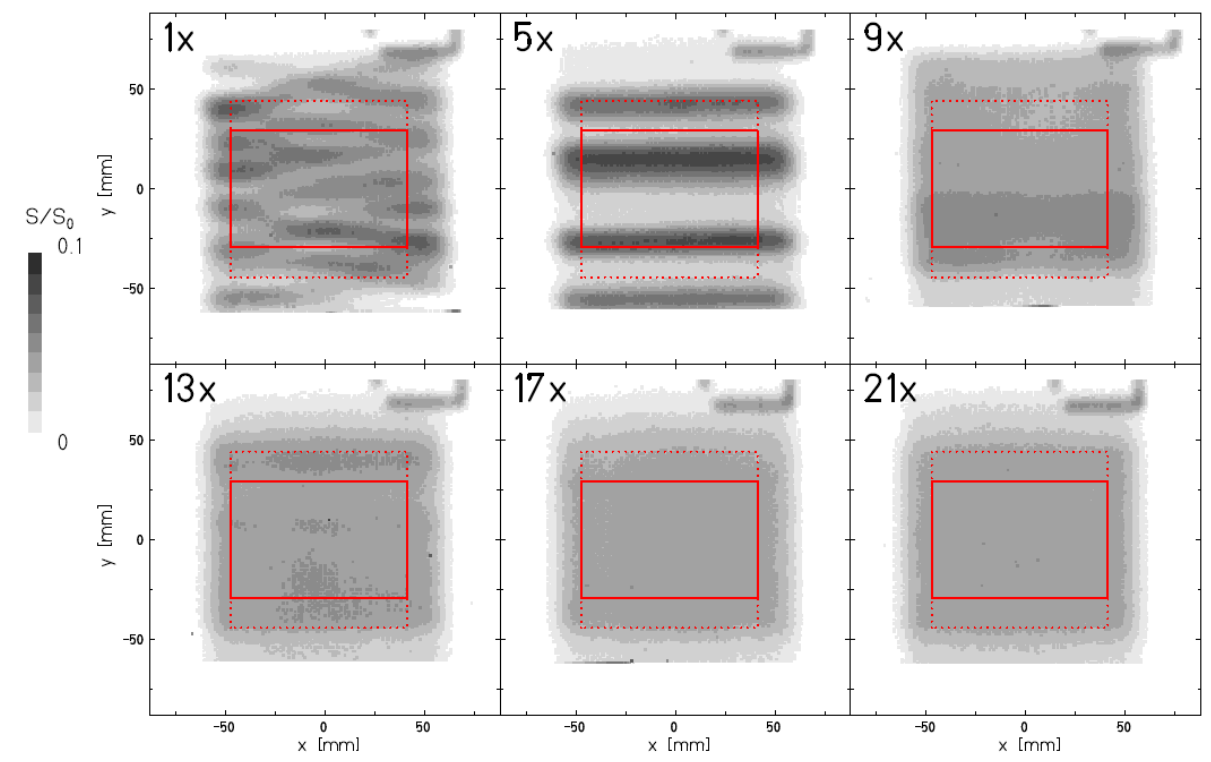

Figure 1.11.: Film irradiation with rescanning. With statistical averaging of multiple interplay patterns the dose in the target (solid red square) becomes homogeneous. Figure taken from [Bert et al., 2009].

Gating applies irradiation only in a selected part of the breathing cycle in a so-called gating window (GW) [Minohara et al., 2000, Lu et al., 2006a]. Usually, the end-exhale position is used as the center of the GW, as highlighted in Fig. 1.12. A motion monitoring signal is used to control beam extraction. While there is limited additional normal tissue irradiation, the treatment time is prolonged due to frequent beam interruptions as shown in Fig. 1.12. Conventional radiotherapy and passive beam shaping also employ gating to reduce the effects of motion on treatment delivery.

Beam tracking is a method where the tumor is followed by the beam throughout different motion phases in real time. Similar to gating, beam tracking is not limited to active beam shaping. It was even proposed originally for photons [Keall et al., 2001] and later implemented clinically in x-ray radiosurgery in the robotic Cyberknife Synchrony system (Accuray Inc., Sunnyvale, Ca., USA) [Brown et al., 2007, Kilby et al., 2010]. Regardless of a radiation type, a fast beam delivery system is required for beam tracking. In contrast to photon radiotherapy, beam tracking with particles need to pay special consideration to range changes. At GSI beam tracking system has been implemented. The solution for fast longitudinal range changes was carried out by two polymethyl methacrylate (PMMA) wedges close to the target, that are operated via a linear step-motor [Saito et al., 2009], as shown in Fig. 1.13. The step-motor can change the relative distance between the wedges and therefore introduces more or less material the beam travels through and consequently changes the effective beam energy (range). The beam position is 


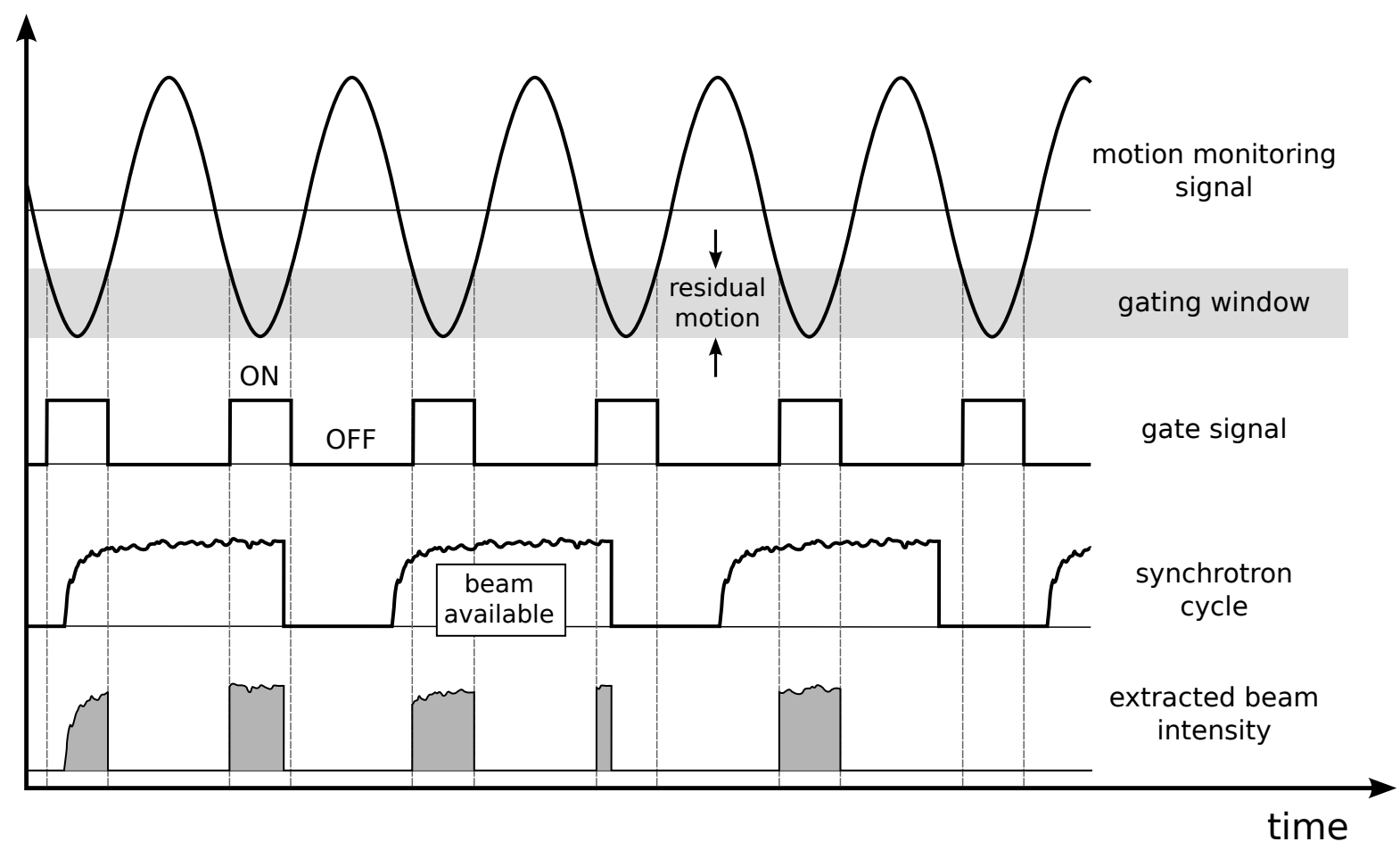

Figure 1.12.: Gating delivery with a synchrotron accelerator. The irradiation is only possible, when the gate signal is active and the beam is available. The gate signal depends on gating window and motion monitoring signal. Figure taken from [Richter, 2012].

corrected according to the motion monitoring signal and pre-calculated look-up tables for the required compensation parameters. The beam tracking system at GSI is able to achieve high precision [Bert and Rietzel, 2007, Bert et al., 2009, Saito et al., 2009]. A clinical implementation, however, is not yet feasible due to several reasons, such as the precision and speed of the motion monitoring, the ion-beam tracking system complexity and inverse interplay.

The three motion mitigation techniques mentioned are not exclusive and can be used in parallel. Furukawa et al. made a study on a combination between rescanning and gating [Furukawa et al., 2007] and Water et al. presented a combination between rescanning and tracking [van de Water et al., 2009]. 


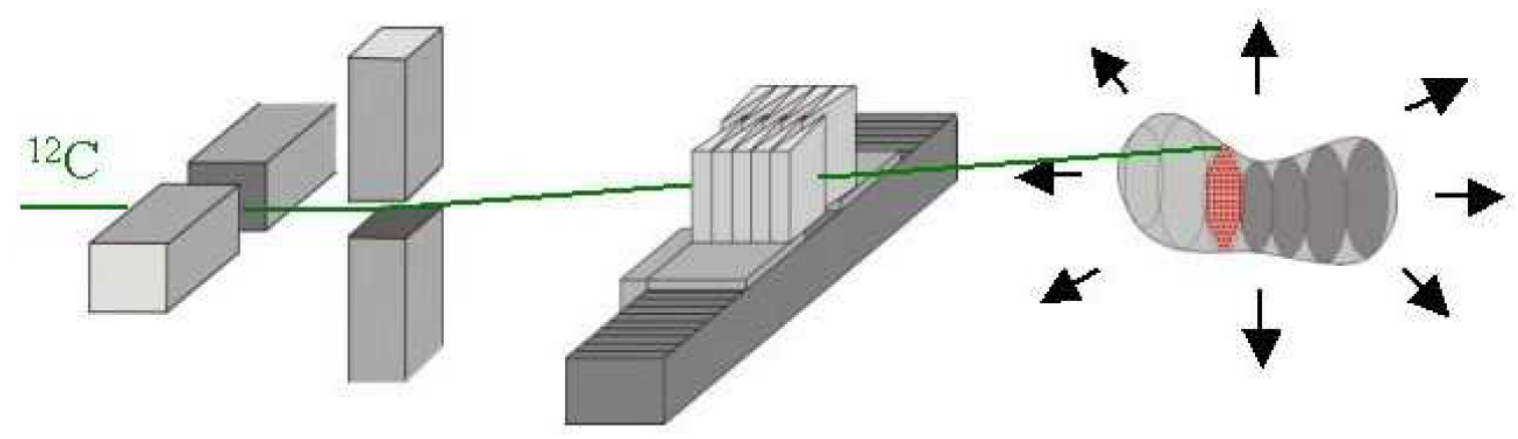

Figure 1.13.: Schematic presentation of GSI's beam tracking system. Two PMMA wedges, mounted on a linear step-motor, can change the energy of the beam traveling through. The changes in the lateral direction are achieved via dipole scanner magnets. For the longitudinal adaptation two PMMA wedges are mounted on a step motors. The thickness of the two PMMA wedges that particle beam travels through is regulated and hence changing its range. Figure taken from [Groezinger, 2004].

\subsubsection{Treatment planning}

The task of treatment planning is to determine machine parameters in order to deliver prescribed dose to the target, while not violating the maximum allowed dose to critical organs, also known as organs at risk (OARs) [Richter, 2012]. Treatment planning thus revolves around the dose optimization process and it is highly dependent on the delivery type used for treatment. The optimization problem for tumors can be written as:

$$
\min _{x} \sum_{i}\left(f\left(x, A_{i}\right)-D_{p r e}\right)^{2}
$$

Here $i$ is a CT voxel, function $f$ is a dose deposition model, $x$ intensity of the radiation beams, A patient geometry and beam parameters and $D_{\text {pre }}$ is the prescribed dose.

The basis of treatment planning is a computed tomography (CT) image, where the target volume and OARs are delineated by a physician. Additional imaging, such as magnetic resonance (MRI) or positron emission tomography (PET), is often used as a supplement to CT for enhanced contrast of the anatomical structures.

\section{Target definition}

The definition of the target volume is crucial, since it has to cover the whole tumor, prevent further tumor spreading, while at the same time it should not be too big to spare normal tissue. The International Commission on Radiation Units (ICRU) recommends the following defini- 
tions for volumes used in treatment planning, which will be used in this work, see Fig. 1.14 [ICRU, 1993a, ICRU, 1999].

Gross Tumor volume: The GTV is the gross palpable or visible/demonstrable extent and location of malignant growth.

Clinical Target Volume: The CTV is a tissue volume that contains a demonstrable GTV and/or subclinical microscopic malignant disease, which has to be eliminated. This volume thus has to be treated adequately in order to achieve the aim of therapy, cure or palliation.

Planning Target Volume: The PTV is a geometrical concept, and it is defined to select an appropriate beam size and beam arrangements, taking into consideration the net effect of all the possible geometrical variations, in order to ensure that the prescribed dose is actually absorbed in the CTV.

Internal Target Volume: This is the margin that must be added to the CTV to compensate for expected physio-logical movements and variations in size, shape, and position of the CTV during therapy.

Organs at risk: Organs at risk (OAR) are normal tissues whose radiation sensitivity may significantly influence treatment planning and/or prescribed dose.

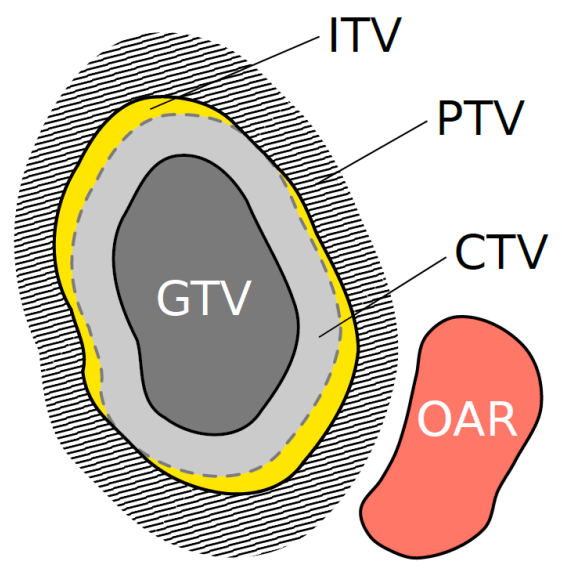

Figure 1.14.: ICRU treatment planning volumes definitions. Figure taken from [Richter, 2012]

Further recommendations of the ICRU state that 100\% of the PTV volume should receive between $95 \%$ and $100 \%$ of the planned dose [ICRU, 1993a].

\section{Treatment planning for scanned ion beams}

A treatment planning system (TPS) for active ion beams shaping has to model the active beam delivery system and the beam interactions with the tissue. Furthermore, for ions heavier than 
protons the biological effectiveness and fragmentation must be considered, which add additional complexity to the TPS. A TPS for beam scanning was developed at GSI, called TRiP98. The basic concepts of TRiP98 will be presented here, further reading can be found elsewhere [Krämer et al., 2000, Krämer and Scholz, 2000, Richter et al., 2013].

TRiP98 divides the PTV into iso-energy slices, which are further divided into raster points in a defined order that the beam will follow. In the optimization step, a gradient-decent algorithm iteratively optimizes particle number for each raster point, so that the optimal target dose is achieved.The dose can either be physical or biological, using the LEM biological effectiveness (see Section 1.2.2). Physical characteristic of the beam include lateral scattering as proposed by Molière [Molière, 1948] and nuclear fragmentation that yield secondary particles. The patient specific geometry and tissue inhomogeneities are accounted for using a transformation from CT HU to water-equivalent path length (WEPL) [Geiss et al., 1999, Jäkel et al., 2001, Rietzel et al., 2007].

\section{GSI's 4D treatment planning system}

As mentioned in section 1.2.4 tumor motion can cause severe dosimetric errors. To asses dose deficiencies and to overcome them, TRiP98 was expanded to be able to calculate time-resolved (4D) treatment plans. The new software was named TRiP4D and a detailed description is given by Richter et al. [Richter et al., 2013].

A static CT is not sufficient for 4D treatment planning. Time-resolved CT scans (4D-CT) therefore have to be used. 4D-CT consist of several quasi-stationary sections, called motion phases. Data is recorded in each slice throughout the whole motion and is then sorted to the appropriate motion phases, according to motion signal [Rietzel et al., 2005a].

Besides a $4 \mathrm{D}-\mathrm{CT}$, a vital part of $4 \mathrm{D}$ treatment planning is image registration. It provides quantification of motion with deformation maps between different 4D-CT motion states. Image registration principles are described in Section 1.2.5. The image registration is not included in TRiP4D, so an external software must provide the necessary deformation maps.

The calculation of a 4D dose starts with the division of the treatment plan into sub-plans, according to the motion phase it will irradiate. The number of sub-plans is the same as the number of motion phases (or the number of motion phases in a gating window, if gating is used). Afterwards the number of particles is calculated in each voxel of all of the motion phases used. Finally, the particle number in each voxel is transformed with the deformation map obtained from registration, to the reference phase, where the accumulated dose from summed particle numbers is calculated (see Fig. 1.15). If a biological dose is calculated, then besides particle numbers, the energy spectra is also accumulated, so that the RBE can be calculated according to LEM for the total dose to each CT voxel. 


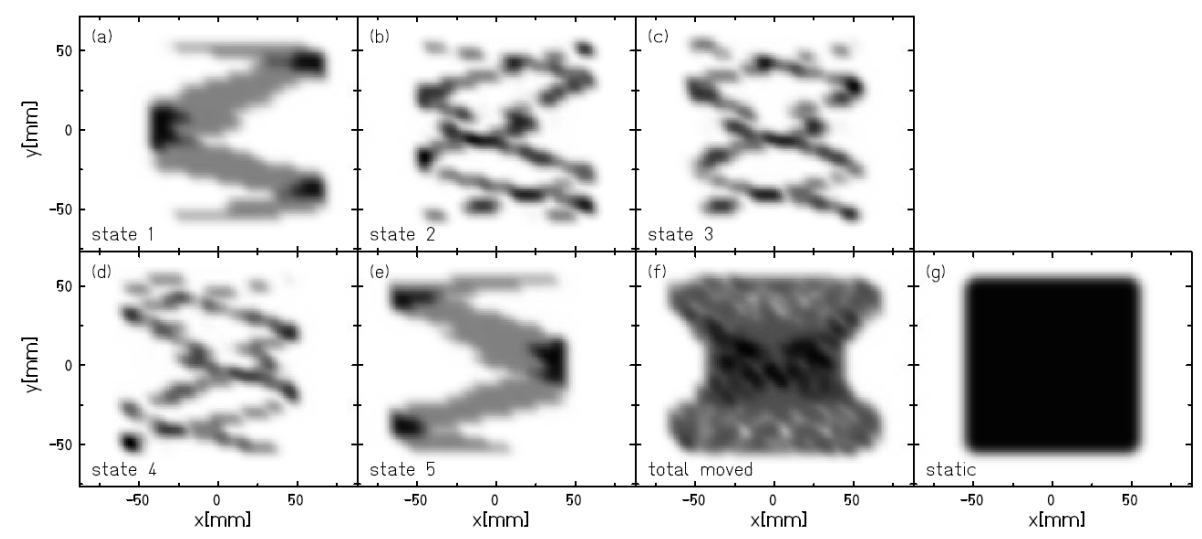

Figure 1.15.: Experimental validation of a TRiP4D dose calculation on film response. On images a)-e) the individual dose deposition for the five motion states is showed. Image $f$ ) shows accumulated 4D dose and image g) a homogeneous dose on a stationary film. Figure taken from [Richter, 2012]

\section{Image registration}

Temporal changes in the patient anatomy are assessed with image registration. The registration can be made between different imaging modalities (CT, MRI, PET), between scans from different days or between different phases in a 4D scan (4D-CT, 4D-MRI, 4D ultra sound). It requires two images: a fixed and a moving one. The result of the registration is a deformation map originating from the moving and pointing to the fixed image. Registration can be written as:

$$
x^{\prime}=x+u_{r i}(x)
$$

Here, $x$ and $x^{\prime}$ are points in states $r$ and $i$, respectively and $u_{r i}$ is a vector field representation of the transformation map. $u_{r i}$ can be used to assess the motion amplitude, propagate contours and calculate the 4D dose. It is important to note that certain steps in 4D treatment planning require also inverse registration, from state $i$ to $r$ [Richter, 2012]. If a deformation map is applied to the moving image, the new image is called warped image and it should be as close to the fixed image as possible. Fixed, moving and warped image are shown in Fig. 1.16. 

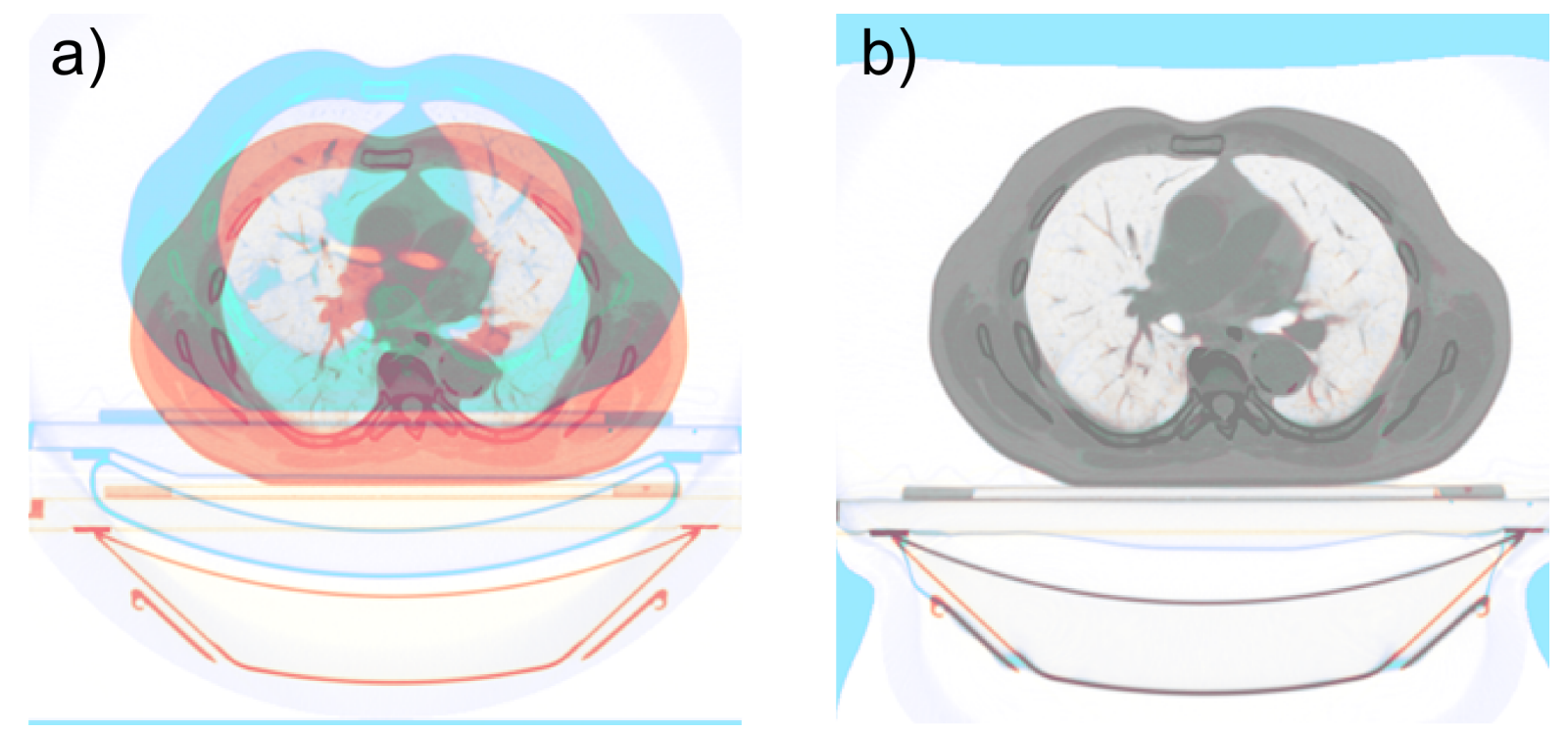

Figure 1.16.: Overlap of a fixed and a moving image CT scan (a) and fixed and warped image after registration (b). Inverse colors are used in both CTs so that the overlayed image should be gray where the images overlap perfectly.

There are different possible types of registration and they can be placed in two groups. The first group consist of linear registrations: translation, rotation and scaling. In medical imaging the most commonly used linear transformation is translation or rigid registration. The other group of registrations are elastic or deformable registrations. The actual algorithms for performing registration are complex and the reader can find a detailed review elsewhere [Hill et al., 2001, Brock et al., 2006, Rietzel and Chen, 2006]. A multi-institutional study has shown that the accuracy for the majority of the algorithms is in the order of the image voxel size, i.e. millimeters [Brock, 2010]. The study further states that the registration quality depends on image contrast. The quality of image registration is actually hard to quantitatively asses and usually the registration results are checked visually. To improve image registration quality assurance a dedicated software was developed and is described in detail in Chapter 2. 


\subsection{Lung cancer}

There were 1.8 million new lung cancer cases diagnosed worldwide in 2012 [WCRF, 2016]. With a very poor prognosis (5-year survival rates in Germany are 16\% for men and 21\% for women [Kaatsch et al., 2014]) lung cancer is one of the most frequent and most deadly cancer types. Usually lung cancer is distiguised into small-cell lung cancer (SCLC) and non-small cell lung cancer (NSCLC). Around 14\% of lung cancer cases are SCLC [Tsao, 2016], which is normally treated with chemotherapy and radiotherapy, while for NSCLC the traditional course of treatment are surgery and radiotherapy. An example of cancerous lung tissue is shown in Fig. 1.17.

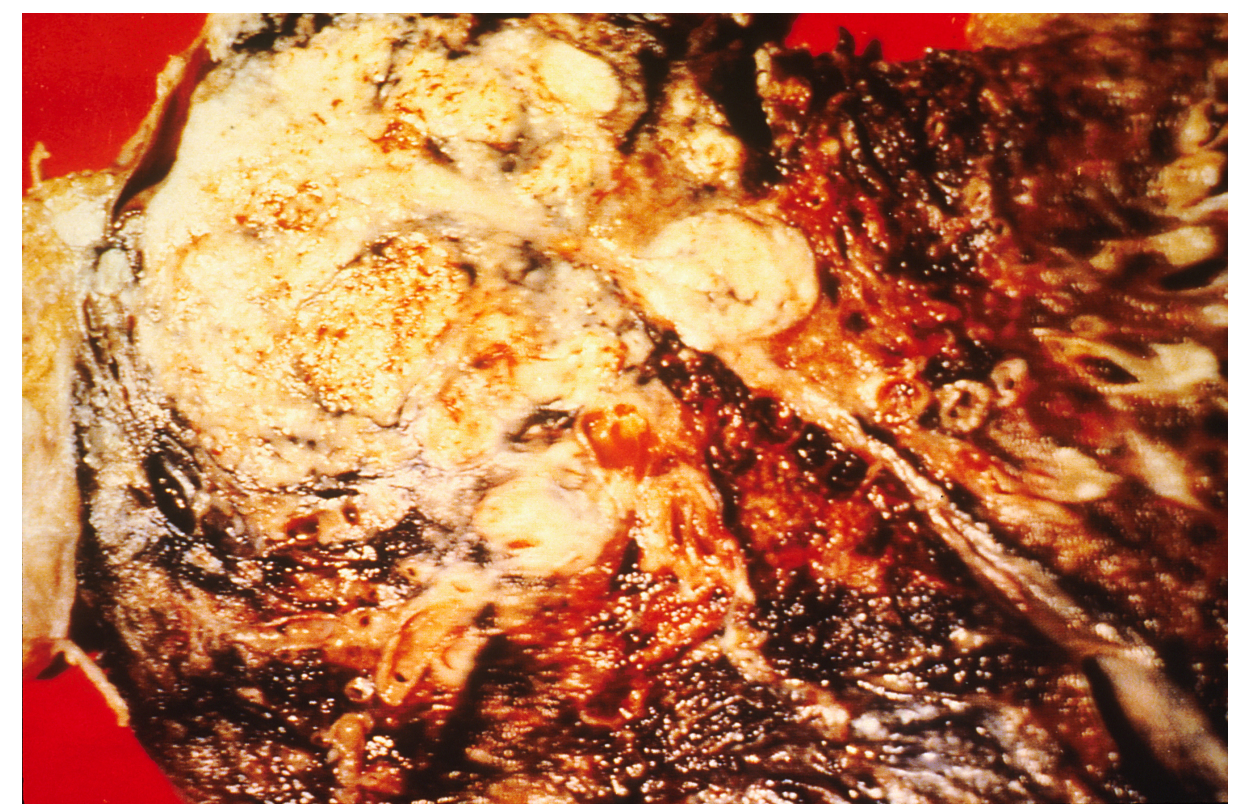

Figure 1.17.: Cross section of a human lung. The white area in the upper lobe is cancerous tissue, while the black areas indicate the patient was a smoker. Figure taken from [NCl, 1988]

\subsubsection{Epidemiology}

The main cause for lung cancer is a long-term exposure to tobacco smoke [Tsao, 2016], with $85 \%$ of cases contributed to smoking. There are over 70 known carcinogens in cigarette smoke, such as benzo[a]pyrene, 1,3-butadiene and a radioactive isotope of polonium, polonium-210 [Hecht, 2012]. See Fig. 1.18 for correlation between sales of tobacco products and rate of the lung cancer.

Studies have shown that passive smokers have an increased risk of lung cancer as well, with more then $20 \%$ increase in risk for those who live with someone who smokes and 16-19\% increase for someone working with a smoker [Taylor et al., 2007]. There is some controversy 
weather smoking cannabis increases risk of lung cancer - two reviews from 2013 and 2014 have found contradicting results [Tashkin, 2013, Underner et al., 2014].

The other $15 \%$ of lung cancer cases is attributed to a combination of genetic factors, exposure to radon gas, asbestos or other forms of air pollution [J. and M., 2010].

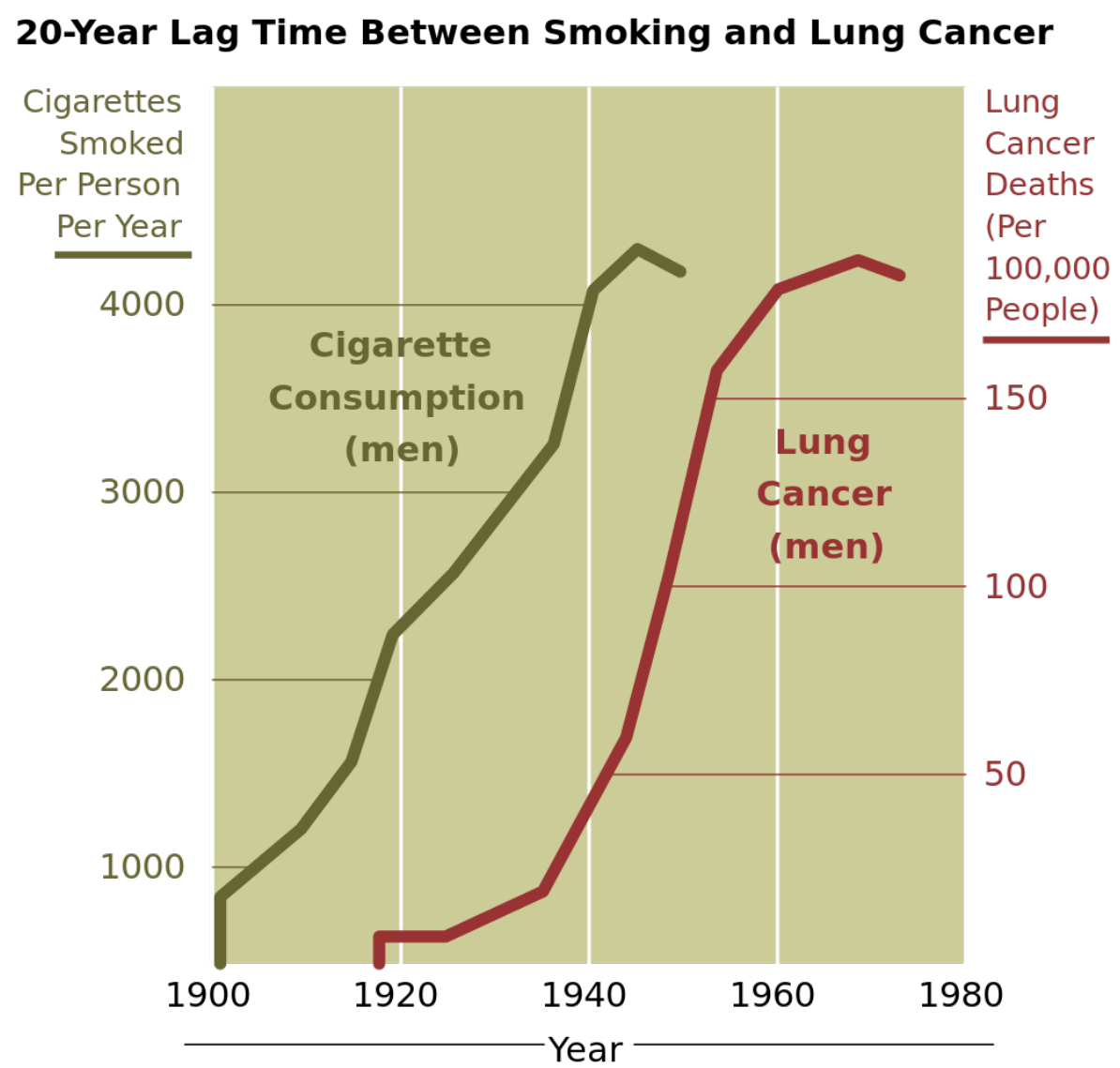

Figure 1.18.: Correlation between sales of tobacco products and the rate of lung cancer in the USA between 1900 - 1970. Data released from National Cancer Institute.

\subsubsection{Non-small cell lung cancer}

The NSCLC is divided into three main groups: adenocarcinoma, squamous-cell carcinoma and large-cell (undifferentiated) carcinoma [Kasper et al., 2015]. Between 25-35\% of all lung cancer cases are adenocarcinoma, squamous-cell carcinoma contributes to around 30\% of lung cases and 10-15\% are large-cell carcinoma. The SCLC contributes the rest.

Lung cancer staging refers to the extent to which the lung cancer has spread from its original source. Additionally, staging is used to establish treatment and prognosis [Chheang and Brown, 2013]. For NSCLC, the TNM classification is used, which depends on size of the primary tumor $(\mathrm{T})$, involvement of the lymph node $(\mathrm{N})$ and distant metastasis $(\mathrm{M})$ [Kasper et al., 2015]. According to the TNM class a group is assigned, from stage 0, IA, IB, IIA, 
IIB, IIIA, IIIB to IV. A schematic presentation of some stages is shown on Fig. 1.19. Prognosis is highly dependent on stage, as shown in Table 1.2 .

Table 1.2.: Five-year survival rates for different stage of NSCLC. Data taken from [Rami-Porta et al., 2009]

\begin{tabular}{|c|c|}
\hline \hline Clinical stage & Five-year survival (\%) \\
\hline IA & 50 \\
IB & 47 \\
IIA & 36 \\
IIB & 26 \\
IIIA & 19 \\
IIIB & 7 \\
IV & 2 \\
\hline \hline
\end{tabular}

(a)

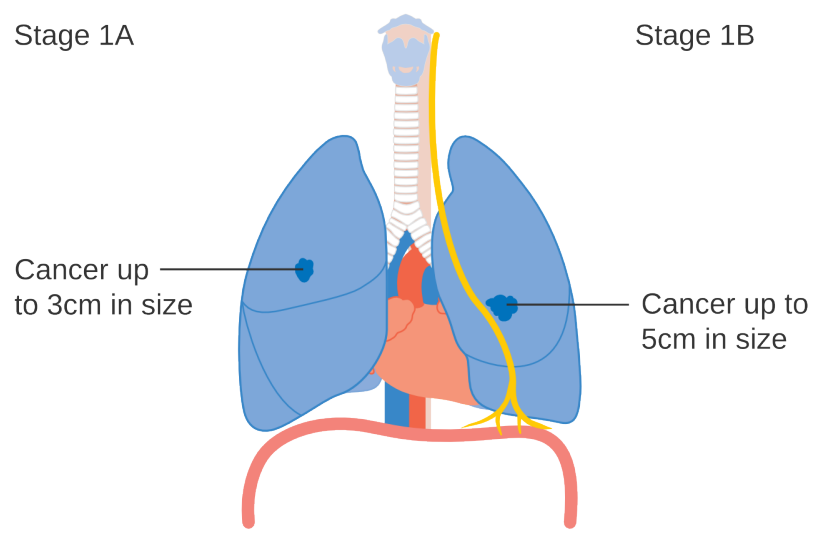

(b)

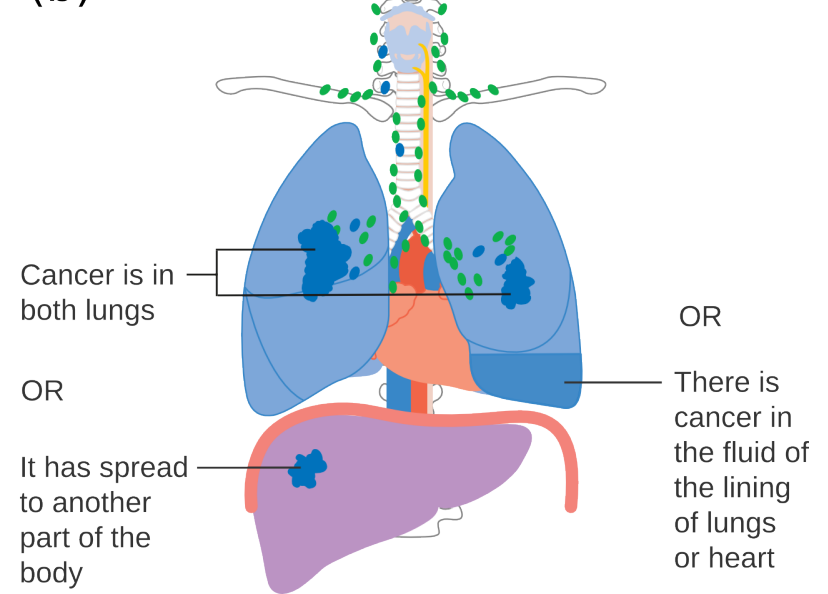

Figure 1.19.: Schematic presentation of three NSCLC stages. (a) Stages IA and IB; (b) Stage IV. Figure taken from [CRUK, 2016] 
The treatment for NSCLC can consist of surgery, chemotherapy, radiation therapy, or a combination of modalities. The treatment course depends on tumor type and stage and on patient overall condition The typical treatment is surgery for stage I and II disease [Tsao, 2016]. Surgery resection consist of either lobectomy or pneumonectomy, together with sampling lymph node or even a complete lymph node dissection. Surgery will only be performed if NSCLC patients have enough lung reserve after lobe or lung is removed. The 5-year survival rate for NSCLC patients undergoing surgery is about 55 to $70 \%$ and $35-55 \%$ for stage I and II disease, respectively.

For unresectable stage III lung cancer the treatment consist of either chemotherapy or radiation therapy or a combination of both. The median survival for patients with unresectable stage IIIA disease is 10-14 months [Tsao, 2016]. For all treated stage IIIB disease the median survival is 7-15 months [K. et al., 2005].

Rather than treating stage IV disease, palliation of symptoms is the goal. With chemotherapy, targeted drugs and radiation therapy the tumor burden can be lessened and the quality of life can be improved. The prognosis is poor, with a median survival of only 9 months and less then $25 \%$ of the patients survive the first year after the disease is diagnosed [Tsao, 2016]. A recent phase II trial combined chemotherapy with stereotactic body radiation therapy and showed promising results with 20 months overall survival [Iyengar et al., 2014]. 


\section{Deformable Image Registration and Validation}

\section{Contents}

2.1. Introduction $\ldots \ldots \ldots \ldots \ldots \ldots \ldots \ldots \ldots \ldots \ldots \ldots \ldots \ldots$

2.2. Implementation $\ldots \ldots \ldots \ldots \ldots \ldots \ldots \ldots \ldots \ldots \ldots \ldots \ldots$

2.2.1. 3D Slicer . . . . . . . . . . . . . . . . . . . . 45

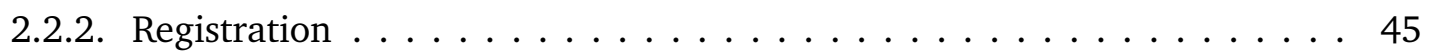

2.2.3. Registration quality tests $\ldots \ldots \ldots \ldots \ldots \ldots$

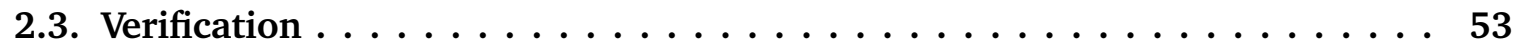

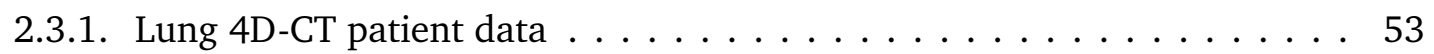

2.3.2. Pig heart 4D-CT data . . . . . . . . . . . . . . . . . . 60

2.4. Summary and Discussion . . . . . . . . . . . . . 68

\subsection{Introduction}

Today most modern clinics regularly use different imaging techniques including CT, 4D-CT, conebeam CT, MRI and PET. A registration is needed to overlay different image acquisitions, such as images taken at different days, between different imaging modalities or to quantify anatomical changes in a time-resolved image acquisition, such as 4D-CT, 4D-MRI or 4D ultra sound. While most commercial treatment planning software provide rigid registration between different images, deformable image registration (DIR) is currently rarely used. DIR can quantify anatomical and biological variations better compared to rigid registration [Sarrut, 2006]. It opens exciting new options in radiotherapy, such as 4D optimization [Trofimov et al., 2005], 4D dose calculation [Flampouri et al., 2006] or contour propagation [Lu et al., 2006b]. 4D dose calculation has been well established for photons [Ong et al., 2016], protons [Paganetti et al., 2005] and carbon-ions [Gemmel et al., 2011] and has received several experimental verifications [Vinogradskiy et al., 2009, Perrin et al., 2016, Bert et al., 2012b].

A 4D dose calculation requires DIR for the deformation of the dose distributions in each motion state to the reference state, where the dose from all motion states is accumulated. 4D dose calculation requires accurate DIR at every voxel, since errors in DIR can significantly alter 
the 4D dose [Heath and Seuntjens, 2006]. Special consideration has to be paid to calculation of biological 4D doses [Gemmel et al., 2011].

Contours can be propagated either with DIR [Lu et al., 2006c, Rietzel et al., 2005b] or with deformable model driven techniques [McInerney and Terzopoulos, 1996], which uses a physical model to iteratively match the contour to image features. In contrast to a $4 \mathrm{D}$ dose calculation, contour propagation requires accurate DIR at the contour boundaries.

Besides radiotherapy, DIR is used also in other medical fields [Cleary and Peters, 2010, Herrell et al., 2012, Nithiananthan et al., 2011, Naini et al., 2010]. Several different DIR algorithms are available, such as B-spline [Rueckert et al., 1999], Demons [Thirion, 1998], linear elastic finite element [Venugopal et al., 2005], optical flow [Zhong et al., 2007] or viscous fluid [Christensen et al., 1996].

The DIR has a large degrees of freedom and as such is an ill-posed problem and hence prone to errors. The errors can result in image misalignments or in physically impossible vector fields. One of the reasons why DIR is not commonly used in commercial softwares is the lack of proper DIR quality assurance (DIRQA), which is essential for implementation of DIR in the clinical work-flow. While several different DIRQA methods exist, none of them are definitive and most of them are time consuming. It is possible to evaluate DIR with deformable phantoms, where the type and size of deformation is known [Kashani et al., 2007, Kirby et al., 2011]. However this effort is prohibitive in the everyday clinical work flow. DIR validation can also be based on landmark positions, specifically their location before and after registration. In absence of externally planted markers, locating landmarks in the patient anatomy can be time-consuming and it can be difficult to identify landmarks in low-contrast regions [Varadhan et al., 2013]. Another option is to compare delineated contours with the propagated ones using the dice similarity coefficient [Varadhan et al., 2013] or Hausdorff distance [Huttenlocher et al., 1993]. While more efficient than landmark checks, these techniques require additional delineation and do not address the region within the contour.

A set of tools was created to systematically handle DIR and DIRQA in the open-source software 3D Slicer. Tools were tested on a large data set to verify their validity. 


\subsection{Implementation}

\subsubsection{D Slicer}

3D Slicer (Slicer) is a software platform for the analysis and visualization of medical images [Slicer, 2016a, Fedorov et al., 2012]. Slicer is a free, open-source software (BSD-style license) available on Windows, MacOSX and Linux operating systems. It comes with a vast variety of tools, such as:

- Handling all commonly image formats, including DICOM, NRRD and MHA

- Visualization of voxel images, polygonal meshes and volume renderings

- Image registration (rigid and non-rigid) and display of vector fields

- Automatic image segmentation

- Analysis and visualization of diffusion tensor image data

- Device tracking for image-guided procedures

The source code of Slicer is written in $\mathrm{C}++$ and with a Python wrapper to provide rapid, iterative development. The graphical user interface is based on Qt. The visualization is based on VTK [Vtk, 2016], a graphical library commonly used in scientific research.

Slicer is a research tool and as such allows implementation of new functionalities in the form of extensions (modules). They can either be as external command-line programs, as scripts to automate Slicer processes or as unique modules with new features.

\subsubsection{Registration}

Plastimatch [Shackleford et al., 2010] is a commonly used software for registration in medical research. It is a free and open-source tool, available as a command-line executable program. Plastimatch B-spline registration is also available in Slicer as part of the extension SlicerRT [Pinter et al., 2012]. The integration of Plastimatch in Slicer brings the advantage of a graphical user interface, offering a quick modification of parameters and visualization of the results. However, automation is needed for a large number of registrations. For a complete 4D-CT registration there are $2(N-1)$ registrations required - from the reference phase to each of $N$ motion states of 4D-CT and vice versa, except for the reference phase itself. Typical 4D-CTs consist of 10 phases, therefore automated registration of a 4D-CT is necessary.

The automated DIR was achieved with a Python class to handle image locations, store DIR parameters, perform DIR in the Plastimatch module, use correct naming conventions and store all output files (vector fields and warped images). Details can be found in Appendix A. 


\section{Registration nomenclature}

To provide a clear and consistent description of methods used, an overview of the expressions is given here.

- Reference image - the image that serves as a reference position in registration (image that is being registered to).

- Moving image - the image that is matched to the reference image (image that is being registered from).

- Warped image - the result of applying a transformation map from registration to the moving image. It should be as close to the reference image as possible.

- True registration - the registration from the moving to the reference image. Similar, everything connected to the true registration will use "true" (true vector field, true warped image, true absolute difference, true Jacobian, etc.).

- Inverse registration - the registration from the reference to the moving image (opposite or inverse of the true registration). As in the true registration, the term inverse can be used for everything connected to it (inverse vector field, inverse warped image, inverse absolute difference, inverse Jacobian, etc.).

In radiotherapy the true registration is used for dose propagation and consequential the 4D Dose calculation, whereas with the inverse registration contours can be propagated from the reference to the moving phase.

\subsubsection{Registration quality tests}

In order to provide visual and quantitative assessment of the registration quality a Deformable Image Registration Quality Assurance or DIRQA module was created. It provides image checks (inverse color, checkerboard, absolute difference, flicker, movie and landmark distances) and vector checks (Jacobian and inverse consistency error). The reference and warped image, true and inverse vector are used as inputs for the DIRQA module. Additionally, landmarks and a region of interest (ROI) can also be used as an input.

Tests can be divided into two groups: qualitative (inverse color, checkerboard, flicker, movie) and quantitative (absolute difference, landmark distances, Jacobian and inverse consistency error) tests. Absolute difference, Jacobian and inverse consistency error tests were build using tools from the ITK library [Yoo et al., 2002]. 


\section{Qualitative tests}

A DIR quality can be assessed with visual inspection of DIR results. Several tests were developed to make the visual inspection easier.

\section{Inverse color}

Overlaying two different images will highlight the difference between them. However, since CT scans are usually displayed in grayscale color code, the differences can be indistinguishable. Especially if the images are quite similar, as the reference and the warped image should be. With applying opposite color codes to overlaying images two things are achieved. First, regions where the registration was successful will be in grayscale. Second, the differences between images will be highlighted in the color of the image they originate from. In the module we used red and cyan color code for reference and warped image, respectively. See Fig. 2.1 for details.

(a)

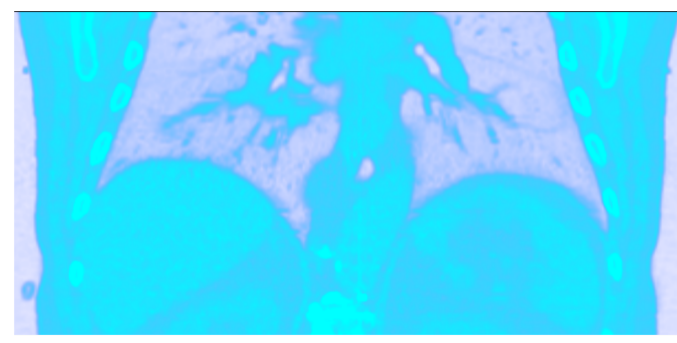

(c)

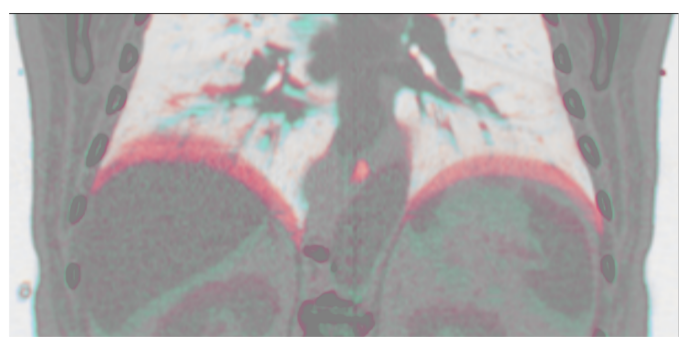

(b)

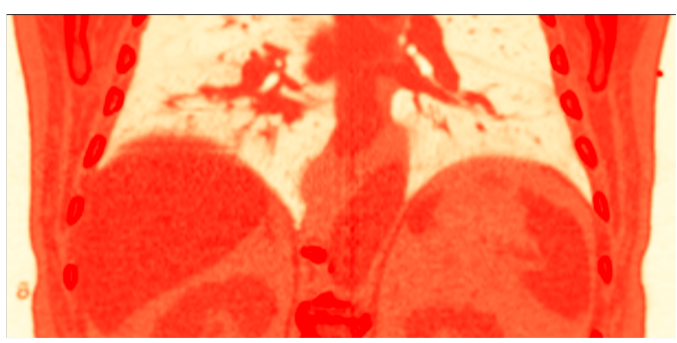

(d)

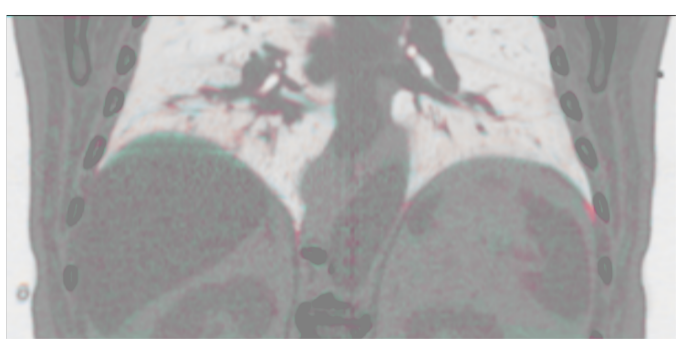

Figure 2.1.: An example of a inverse color overlay. Images (a) and (b) show red and cyan color code, respectively, for a CT scan. (c) displays overlayed inverse colored images before and (d) after registration.

\section{Checkerboard}

As the name suggests, checkerboard creates an image of tiles. Each tile alternates between the reference and the warped image, as shown in Fig. 2.2. The differences between two images become apparent if there is no smooth transition from one tile to the next. The number of tiles can be manually selected. 
(a)

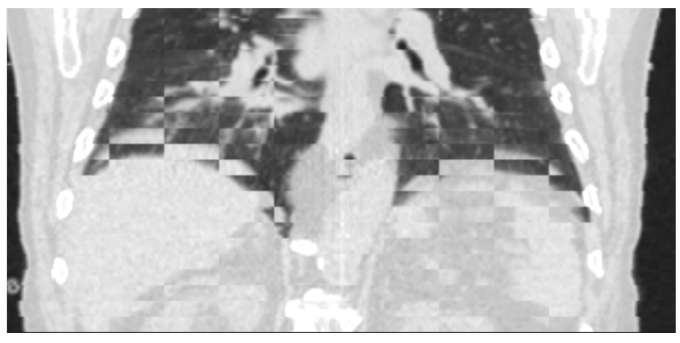

(b)

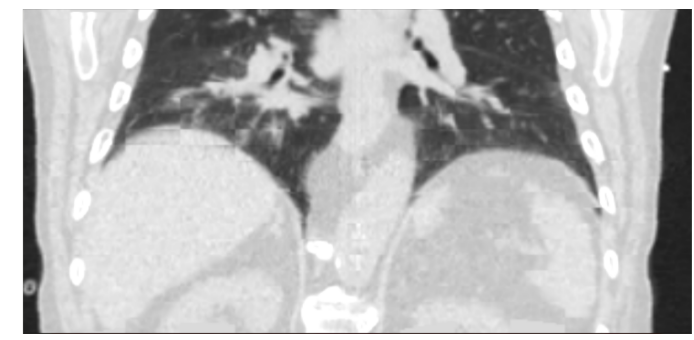

Figure 2.2.: An example of a checkerboard image. It consists of tiles alternating between two images. Tiles in image (a) alternate between scans before registration and in image (b) after registration.

Movie

Medical images are usually quite large - a typical CT image consist of $512 \times 512 \times 100$ pixels, which makes inspecting image checks (inverse color, checkerboard, absolute difference) a timeconsuming task. The movie feature allows a smoother display of different image slices. The user selects which view to inspect (axial, sagital or coronal) and presses start. Selected views then start scrolling from the first to the last slice of selected view. It allows user to focus on registration details, rather than scrolling through slices.

\section{Flicker}

While it is possible to display two images side by side in Slicer, it can sometimes be hard to see fine differences between the two images. Flicker alternates between reference and warped image on a single display at a $0.5 \mathrm{~s}$ rate. It allows a quick inspection of differences between two images in their original form.

Movie and flicker (explained in the next section) do not offer any specific registration checks, but improve the process of DIRQA. 


\section{Quantitative tests}

It can be hard to visually inspect all the DIR results, especially in a 4D-CT registration. Therefore a series of tests were developed that give a quantitative assessment of DIR quality.

\section{Absolute difference}

To stress the difference between reference and warped image an absolute difference between voxel values is calculated and displayed. A new image is generated with voxels populated as the absolute difference between reference and warped image voxel values, as shown in Fig. 2.3. Furthermore, statistical values of absolute differences are calculated (mean, standard deviation, minimum and maximum) for a quantitative assessment of the registration quality (in the ideal case all values would be 0 ).

Three absolute differences can be calculated: between reference and moving image, called default absolute difference; between true warped and reference image, called true absolute difference; between inverse and moving image, called inverse absolute difference. Usually, registration works on minimizing the absolute difference (mean square error metric), so it is a direct indicator of registration success.

To save computational time or to focus on a specific region, the absolute difference can also be calculated just on a specific ROI (if used as an input). Usually the patient body is selected as ROI, without air and couch.

(a)

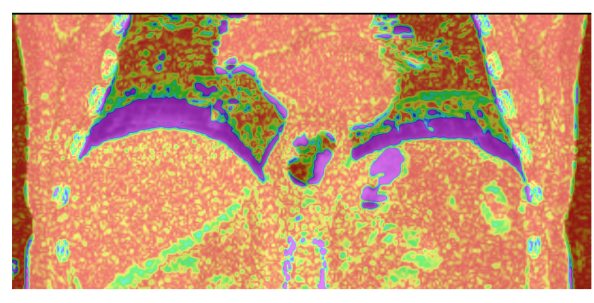

(b)

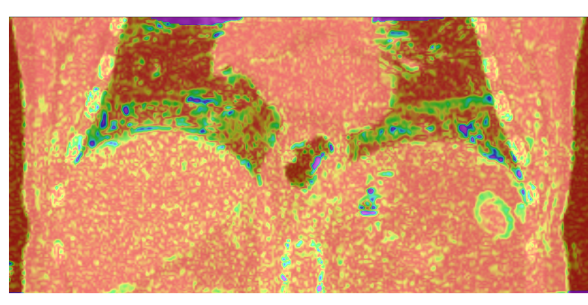

Legend (HU):

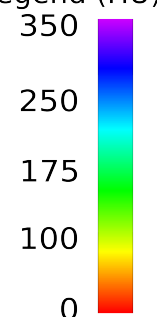

Figure 2.3.: An absolute difference image before (a) and after registration (b). The mean absolute difference before registration (default absolute difference) is $62 \mathrm{HU}$ and $31 \mathrm{HU}$ after registration (true absolute difference). 
Distances between landmarks before and after registration are often used to determine the spatial accuracy of registration [Castillo et al., 2009]. Landmarks can either be a specific feature in the patient anatomy or an external marker. The position of landmarks in the warped image would ideally be at the same position as in the reference image. The module measures the Euclidean norm between the landmark positions in reference and warped image.

The user has to manually select landmarks in reference and moving or also in the warped image. For landmarks based on the patient anatomy, a selection from physician is required. Landmarks from the moving image can then be automatically transformed to the warped image using the registration vector field. An example is shown in Fig. 2.4.

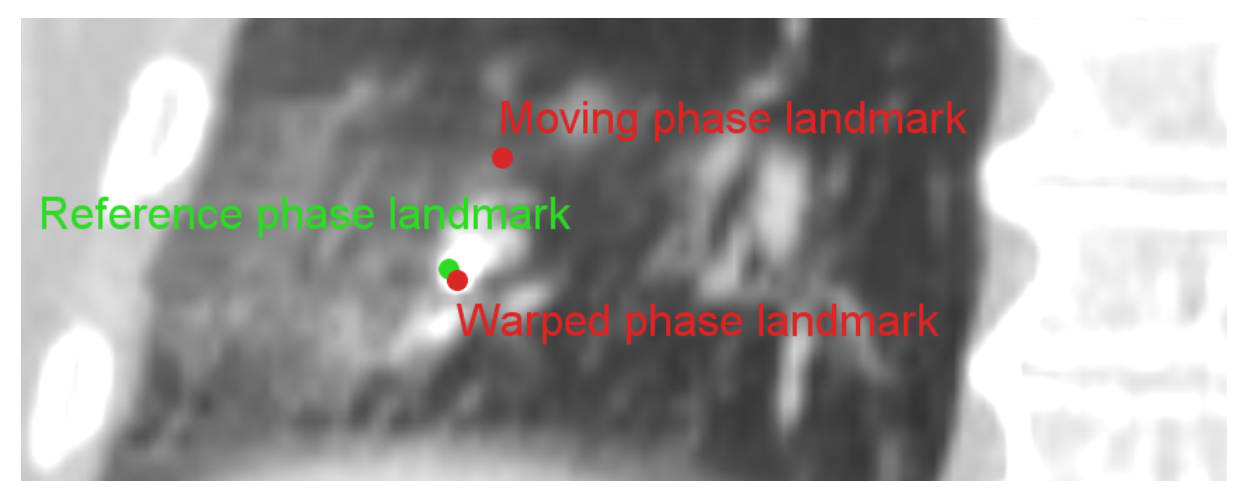

Figure 2.4.: A display of landmarks in three phases - reference, moving and warped phase. The distance before registration (between reference and moving landmark) is $22 \mathrm{~mm}$ and after registration (between reference and warped landmark) is $2 \mathrm{~mm}$. 
The Jacobian determinant or Jacobian $(J)$ of the vector field $u$ is calculated as:

$$
J=\left|\begin{array}{lll}
\frac{\partial u_{x}}{\partial x} & \frac{\partial u_{x}}{\partial y} & \frac{\partial u_{x}}{\partial z} \\
\frac{\partial u_{y}}{\partial x} & \frac{\partial u_{y}}{\partial y} & \frac{\partial u_{y}}{\partial z} \\
\frac{\partial u_{z}}{\partial x} & \frac{\partial u_{z}}{\partial y} & \frac{\partial u_{z}}{\partial z}
\end{array}\right|
$$

The Jacobian is used to validate the physical behavior of the registration [Leow et al., 2007]. The Jacobian of a vector field should be positive, since negative Jacobian values correspond to organ folding, which is physically unrealistic for patient anatomy [Rey et al., 2002, Chen et al., 2008]. Expansions and contractions around a point are indicated by Jacobian values greater or lower than 1 , respectively.

The DIRQA module calculates and displays the Jacobian of the vector field, as shown in Fig. 2.5. Statistical values of the Jacobian are also calculated. Similar to the absolute difference, the Jacobian can be calculated inside a ROI rather than on the whole image.
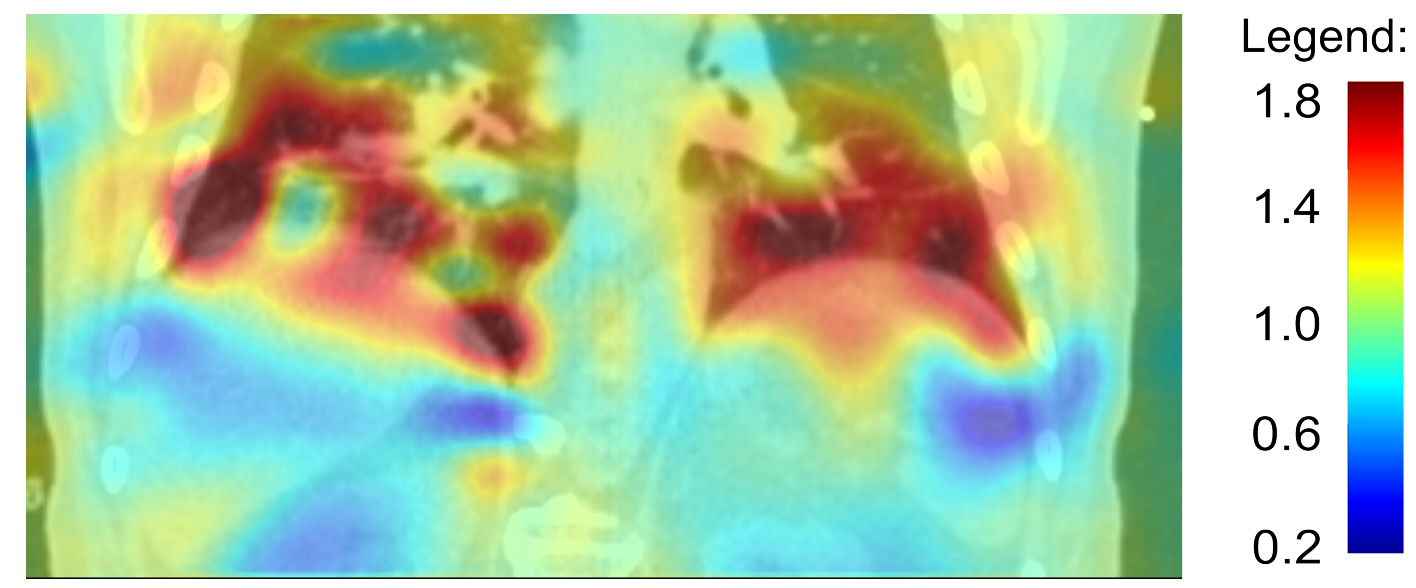

Figure 2.5.: An image of the Jacobian values overlayed on a CT scan. The average value of the displayed Jacobian is 0.98 with 0.09 standard deviation. 
The inverse consistency error (ICE) is consistently used in literature as one of the main vector field checks [Christensen and Johnson, 2001, Bender and Tomé, 2009]. The principle is as follows. Suppose we have two vector fields: $u_{A B}$ obtained from registration of image $A$ to $B$ and $u_{B A}$ from registration of image $B$ to $A$. The two registrations are performed separately. In an ideal scenario, $u_{A B}$ would be a direct inverse of $u_{B A}$. However, DIR algorithms do not yield perfectly inverse consistent vector fields. Therefore, the differences between true and inverse vector fields have to be examined.

To check for ICE, an algorithm was created that first transforms a point $x$ in image $A$, using $u_{A B}$. The newly obtained point $x^{\prime}$ is then transformed with the inverse vector field, $u_{B A}$ which yields $x^{\prime \prime}$. The ICE is defined as Euclidean norm between $x$ and $x^{\prime \prime}$ :

$$
I C E=\left|x-x^{\prime \prime}\right|=\left|x-u_{B A}\left(x^{\prime}\right)\right|=\left|x-u_{B A}\left(u_{A B}(x)\right)\right|
$$

Points $x^{\prime}$ and $x^{\prime \prime}$ can have an arbitrary position in space, while vector fields $u_{A B}$ and $u_{B A}$ are positioned on a grid. To apply the transformation $u_{B A}\left(x^{\prime}\right), x^{\prime}$ is interpolated on a $u_{B A}$ grid. A tri-linear interpolation is used in this module.

As for the Jacobian, ICE image is calculated and displayed, along with statistical values and ROI feature. An example is shown in Fig. 2.6.
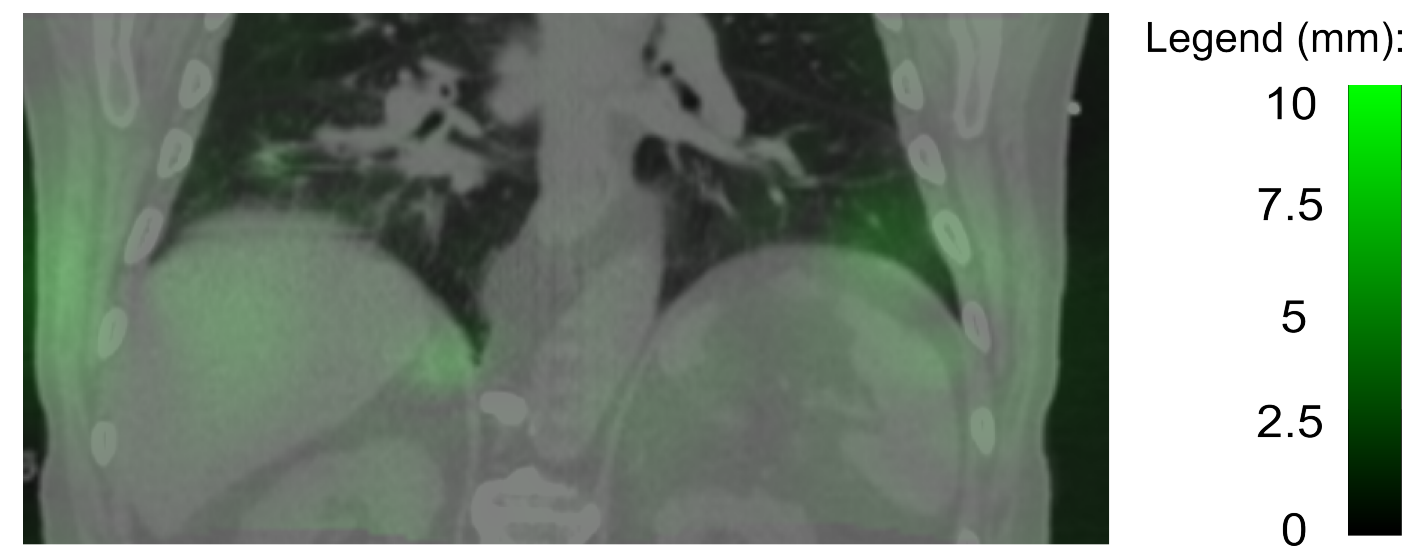

Figure 2.6.: An image of the inverse consistency error (ICE) overlayed with CT scan. The average value of displayed ICE is $1.0 \mathrm{~mm}$ with $0.8 \mathrm{~mm}$ standard deviation. 


\subsection{Verification}

Several tools to perform DIR and DIRQA were created. To prove their functionalities, we have tested them on two sets of an actual clinical data. First were the lung 4D-CT patient data from the Champalimaud Center for the Unknown, Lisbon (Portugal). The 4D-CTs were used in the treatment planning studies, presented in Chapters 3 and 4. DIR resulting from 4D-CTs played an essential in these studies, since they were used in contour propagation to estimate and mitigate tumor motion. In addition, DIR was also used in 4D dose calculation.

The second set of data were pig cardiac 4D-CTs. They were also used in treatment planning as a part of an animal study, conducted at GSI. 4D-CT DIR was also used for contour propagation and 4D dose calulation. However, the treatment plans were than actually used for the animal irradiation. It was therefore necessary not only to obtain DIR, but also to ensure its quality.

The DIRs were calculated as explained in Section 2.2.2. Afterwards absolute difference, Jacobian and ICE were calculated on resulting DIR as part of the the DIRQA.

\subsubsection{Lung 4D-CT patient data}

The effects of interplay between tumor motion and active beam scanning can drastically change the dose distribution for PT. It is therefore necessary to estimate tumor motion and employ designated motion mitigation techniques.

Chapters 3 and 4 present studies on simulating active scanning carbon ion treatment for nonsmall cell lung cancer patients. The studies included data for 23 lung cancer patients. In order to calculate realistic treatment plans, 4D-CT scans were used in studies. All 4D-CT were registered to estimate and mitigate tumor motion and to calculate 4D doses. To ensure the DIR quality, DIRQA was performed on all DIRs.

\section{Materials and Methods}

In total, 23 lung cancer patients were studied. For each patient, a time-resolved CT (4D-CT) was acquired, consisting of 10 motion states (0 - 9) with $1 \mathrm{~mm}$ pixel and 1-2 mm slice spacing. They were acquired with either a Philips Brilliance BigBore 16-slice (Philips Healthcare, Eindhoven, Netherlands) or a Philips Gemini PET-CT 16-slice scanner. State 0 and 5 correspond to the end-inhale and end-exhale breathing state, respectively. State 0 was chosen as a reference state.

True and inverse DIRs were computed for each patient between each state and the reference state. Each 4D-CT required 18 DIRs, leading to 414 DIRs in total.

The B-Spline Plastimatch module in Slicer was used for DIR (see Section 2.2.2). DIRs were done in two stages with details given in Table 2.1. 
Table 2.1.: Parameters used for B-Spline Plastimatch DIR. A mean squared error metric was used. Details for each parameter can be found in [Plastimatch, 2016].

\begin{tabular}{|c|c|c|}
\hline \hline Parameter & Stage 1 & Stage 2 \\
\hline Resolution & $4,4,2$ & $1,1,1$ \\
Grid size & 50 & 15 \\
Regularization lambda & 0.005 & 0.005 \\
Iterations & 200 & 100 \\
\hline \hline
\end{tabular}

A box-shaped ROI around the patient body was created with a Slicer build-in function. The ROI was employed in calculation of absolute difference, Jacobian and ICE.

Default, true and inverse absolute difference were calculated. In total 621 absolute differences were calculated. All images were down-sampled by a factor of 2 before calculation of absolute difference to save computer time. Similarly, 414 vector fields were down-sampled by a factor of 2 before calculating Jacobian and ICE. Jacobian and ICE checks were calculated on all vector fields. Additionally, vector field magnitudes were analyzed for mean, standard deviation (STD) and maximum (max) values. Paired t-tests were performed to compare mean, STD and max of true and inverse vector field magnitudes. A p-value $<0.05$ was considered significant. A Pearson's $r$ coefficient was used to determine linear fit quality.

For each patient it took around $20 \mathrm{~min}$ for all 18 DIRs and around $30 \mathrm{~min}$ for complete DIRQA on the 9 motion states. A cluster of different Linux computers, each with 8 CPU and 32 GB RAM were used for DIR and DIRQA.

DIR was used in treatment planning, specifically in contour propagation and 4D dose calculation. The areas with poor DIR were investigated and distance between DIR errors and target contour was measured. If the target and the beam path was more than $5 \mathrm{~cm}$ away from DIR errors, DIR was not repeated. 

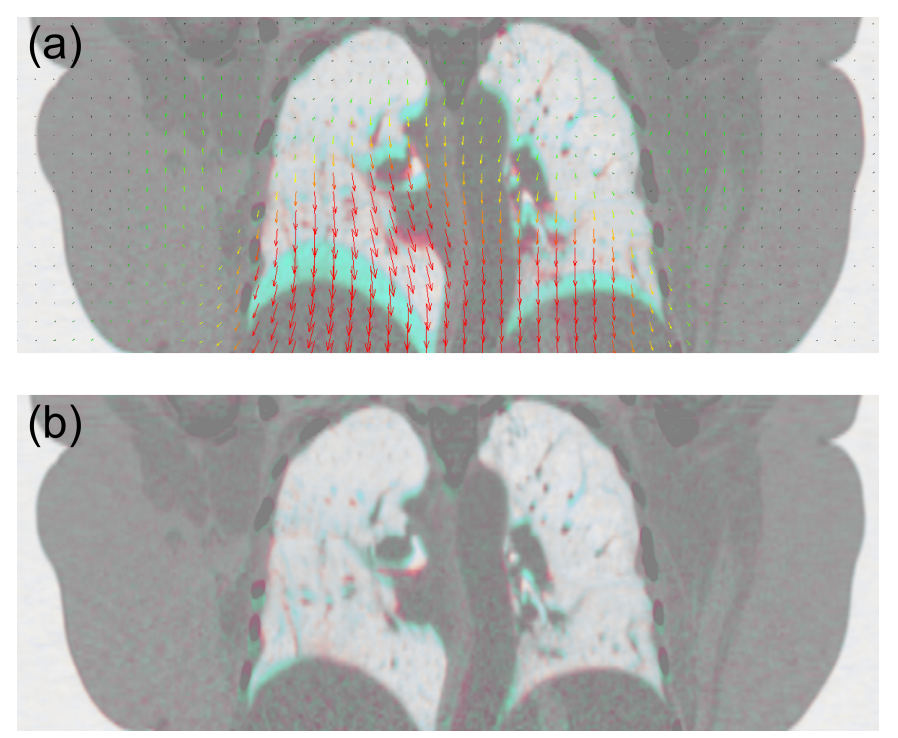

Figure 2.7.: Inverse color overlay of two states before (a) and after (b) DIR. Vector field is displayed on image (a) as arrows.

Results

An example of a DIR is displayed in Fig. 2.7. The statistical analysis of vector fields is shown in Table 2.2. No statistical difference between true and inverse vector field magnitudes was found. The biggest contribution to vector field magnitude was from superior-inferior direction (around $50 \%$ ), followed by anterior-posterior direction (around 30\%) by left-right direction (around $20 \%)$.

Table 2.2.: Data of vector magnitudes. Values are presented as mean (range).

\begin{tabular}{|c|c|c|}
\hline \hline & True vector field $(\mathrm{mm})$ & Inverse vector field $(\mathrm{mm})$ \\
\hline Mean & $0.38(0.01-1.28)$ & $0.38(0.01-1.3)$ \\
STD & $0.95(0.04-3.17)$ & $0.98(0.04-3.55)$ \\
Max & $9.67(0.61-28.56)$ & $10.17(0.56-37.11)$ \\
\hline
\end{tabular}

The correlation between absolute difference before (default) and after DIR (true and inverse) is shown in Fig. 2.8. It also shows default absolute difference distribution across 9 states.

Distribution of true and inverse Jacobian and ICE data are displayed in Fig. 2.10.

True and inverse maximum and minimum Jacobian and maximum ICE values were plotted against maximum vector magnitudes and fitted with linear function. The results are shown in Fig. 2.9.

Linear fits used in Fig. 2.8 and 2.9 were statistically significant ( $\mathrm{p}<0.05)$.

All areas with poor DIR were further than $5 \mathrm{~cm}$ away from the target and no repetition of DIR was necessary. 

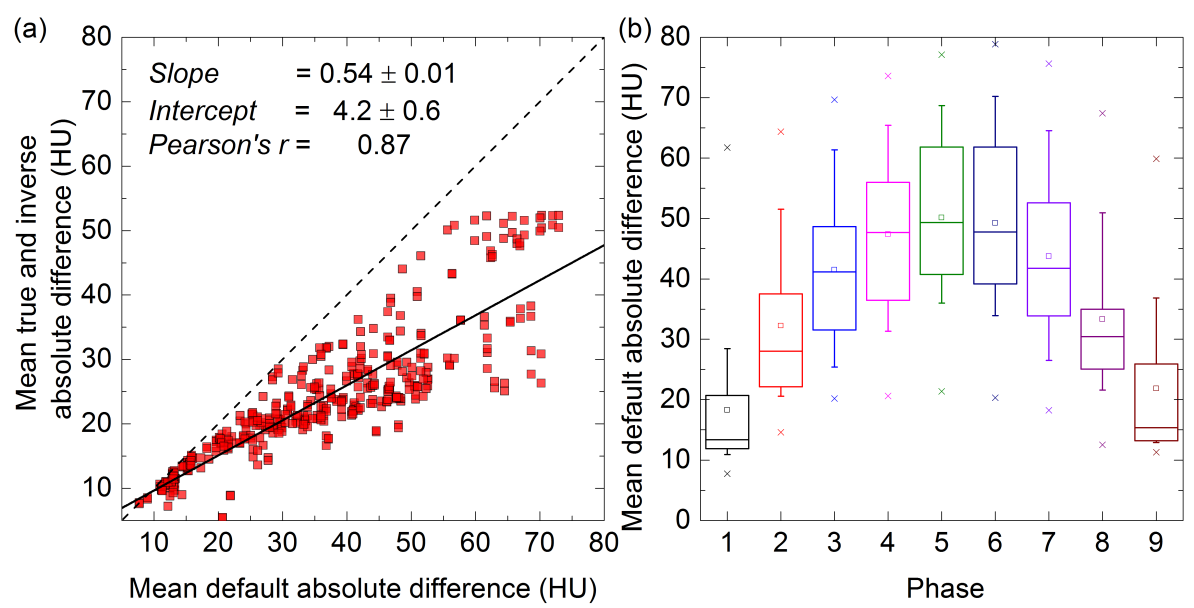

Figure 2.8.: (a) The mean true and inverse absolute difference plotted against the mean default absolute difference. The solid line shows a linear fit, with parameters written in the top left corner. Dashed line shows $y(x)=x$. (b) Box plots of mean default absolute difference distribution across nine 4D-CT states. Boxes represent 25-75\%, whiskers $10-90 \%$ of data. The median is shown with a solid line, the mean is represented with squares and outliers with crosses.
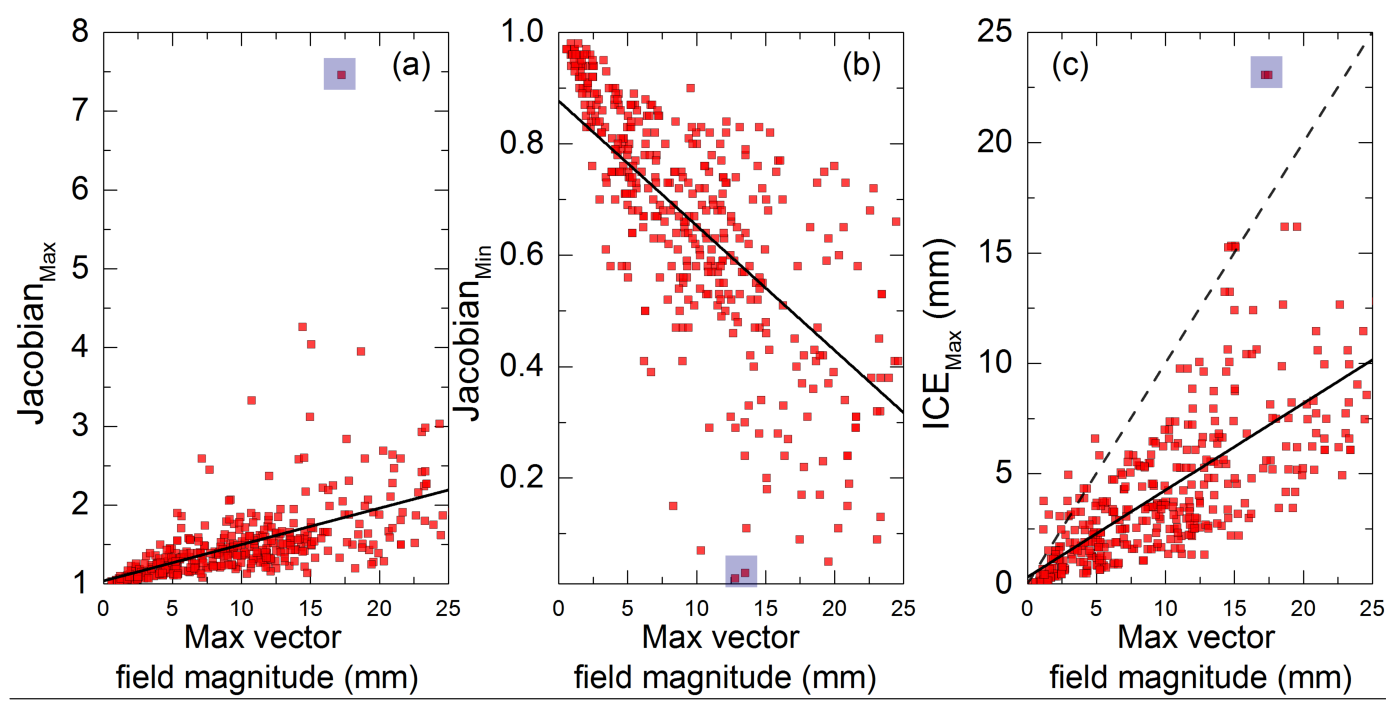

$$
\begin{array}{lccc} 
& \text { Jacobian }_{\text {Max }} & \text { Jacobian }_{\text {Min }} & \text { ICE }_{\text {Max }} \\
\text { Slope } & =0.05 \pm 0.002 & -0.02 \pm 0.001 & 0.39 \pm 0.02 \\
\text { Intercept }= & 1.04 \pm 0.04 & 0.88 \pm 0.01 & 0.31 \pm 0.22 \\
\text { Pearson's } r= & 0.58 & -0.69 & 0.74
\end{array}
$$

Figure 2.9.: Values of maximum Jacobian (a), minimum Jacobian (b) and maximum ICE (c) plotted against the maximum vector magnitudes. A linear fit is displayed with a solid line and parameters are written below the plots. The dashed line in (c) shows $y(x)=x$ plot. The points highlighted with blue squares come from the same DIR error that is shown in Fig. 2.11 

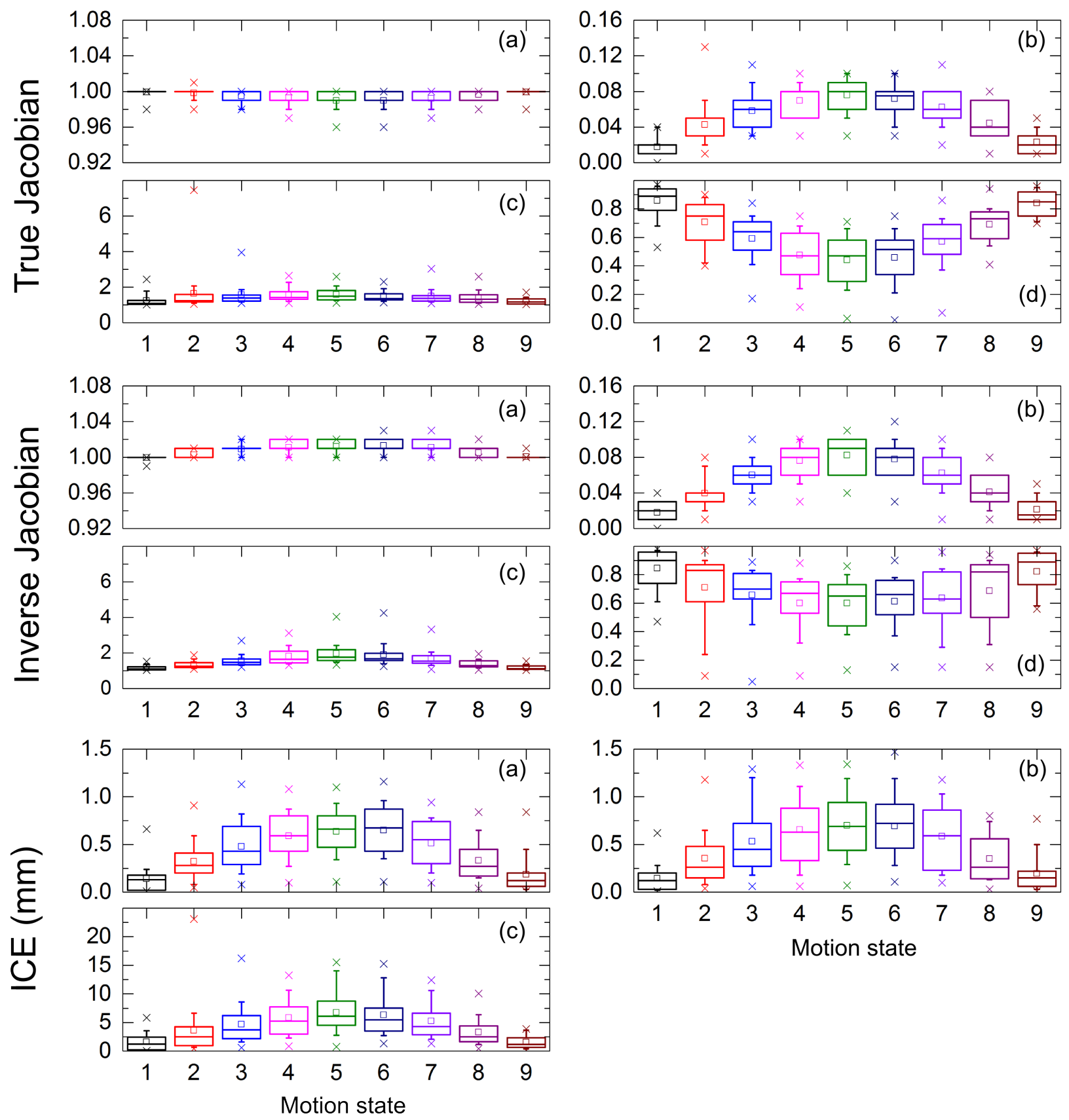

Figure 2.10.: Data for the true (top) and inverse (middle) Jacobian and ICE (bottom) for 9 4D-CT states (reference state 0 is excluded) for 23 lung cancer patients. Mean, STD, maximum and minimum are represented as (a), (b), (c) and (d), respectively. Minimum ICE is 0 throughout all states and patients. Boxes represent $25-75 \%$, whiskers $10-$ $90 \%$ of data. The median is shown with a solid line, the mean is represented with squares and outliers with crosses. 


\section{Discussion}

Nine states of 4D-CTs were registered to a 4D-CT reference state for 23 lung cancer patients, producing 414 true and inverse vector fields. All 414 DIR underwent a DIRQA consisting of vector field magnitudes, absolute difference, Jacobian and ICE.

Vector field magnitudes confirm previously published data that the biggest motion for lungs is in superior-inferior direction [Seppenwoolde et al., 2002, Britton et al., 2007, Liu et al., 2007]. The mean vector field magnitude is small (in submilimeter range), because the ROI included the whole patient body, not just the lungs where most of the motion occurs. Vectors and inverse vectors are similar, which was expected.

There was a high correlation (Pearson's $r=0.87$ ) between absolute difference before and after DIR. The slope of the linear fit suggests that the B-Spline DIR on average halves the absolute difference. There are several outliers from the linear fit for default absolute difference bigger than $50 \mathrm{HU}$, however no DIR errors could be found upon visual inspection for this outliers. All absolute differences after the DIR are smaller then before, which is a necessary condition in order for DIR to be considered successful. Apart from smaller absolute difference after DIR, nothing could be deducted about the DIR quality from the absolute difference. The absolute difference is limited by the image noise, which will always be present in a CT scan [Polacin et al., 1992]. It would be interesting to study the correlation between image noise, absolute difference and consequential DIR quality.

Due to small mean vector field magnitudes, average values for true and inverse Jacobian were $1 \pm 0.05$, which indicates that most of the patient body does not change during the 4D-CT scan. However, patients expansions and contractions can be seen on maximum and minimum Jacobian, with average values around 1.50 and 0.65 respectively.

ICE Mean and STD values were in submilimeter range, due to the correlation between vector field and ICE (see Eq. 2.2). The maximum ICE $(2.3 \mathrm{~cm})$ was observed in a patient with an artifact present in state 2 of the 4D-CT, as shown in Fig. 2.9.

Large vector field magnitudes will produce more errors in DIR as shown in Fig 2.9. Linear fits were used to estimate the increase (decrease) of the Jacobian and ICE. As a rough DIRQA check, ICE should always be smaller than the maximum vector field magnitude. To confirm this, all cases above the dashed line in Fig 2.9c were investigated. For all areas of poor DIR were found. An extreme case (highlighted in Fig. 2.9a-c) had a large image artifact present in states 2 and 3 (state 3 with ICE is shown in Fig. 2.9d) leading to large inconsistencies in DIR. The effect of DIR inconsistency on contour propagation can be seen in Fig. 2.11, where lungs and liver contour were propagated using DIR. The propagated contours clearly differ from the image features.

The 4D-CT DIRs investigated here were used in particle therapy treatment planning. All areas that were found to have a poor DIR, were so far away from the target, that contour propagation or 4D dose calculation were not affected. Hence a repetition of DIR was not necessary. The 
patient in Fig. 2.11a had a tumor in the upper left lung lobe and DIR inconsistencies were found in lower right lung lobe, as can be seen in Fig 2.11b. Therefore all DIRs were considered as successful.
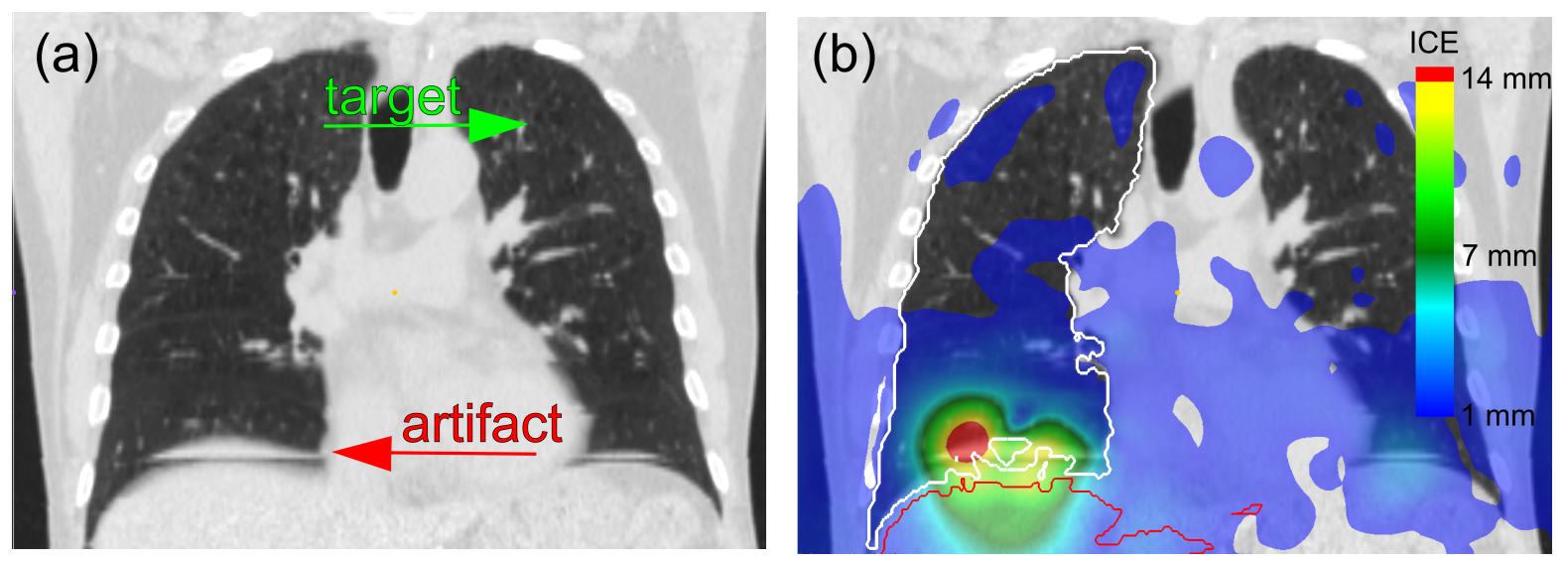

Figure 2.11.: Example of a contour propagation with an inconsistent DIR.(a) shows the location of 4D-CT artifact (red) and the location of the target (green). The distance between them is big enough, that artifact does not affect treatment planning. The large ICE is shown in (b) along with a propagated lung and liver contour. The errors in DIR result in a non-continuous diaphragm. Lungs are outlined in white and liver in red. 


\subsubsection{Pig heart 4D-CT data}

Atrial fibrillation is the most common type of cardiac arrhythmia, causing a quivering motion of the atrial small heart chamber. Although atrial fibrillation directly is not a life threatening condition, it worsens the patient's quality of life and increases the risk of a stroke [Benjamin et al., 1998]. A common method for treating the atrial fibrillation is a catheter ablation [January et al., 2014]. The success rate of a catheter ablation is still limited and can lead to major complications or even death of a patient [Cappato et al., 2005, Cappato et al., 2010].

As an alternative treatment, carbon-ion therapy was proposed [Bert et al., 2012a] and later the feasibility was shown on a beating heart experimentally [Lehmann et al., 2015]. In 2014 a pilot experiment was performed at GSI using large animal model (pigs) and scanned carbon-ion to verify the treatment in vivo [Graeff et al., 2015].

To estimate and compensate motion of the heart during irradiation DIR of 4D-CT data was required. Furthermore, because of the actual irradiation of pigs a DIR quality had to be estimated and repeated, if necessary.

Materials and Methods

\section{Pig irradiation experiment}

DIR and DIRQA procedures will be given here, while a detailed description of the whole pig irradiation experiment can be found elsewhere [Graeff et al., 2015]. Cardiac gated contrastenhanced CT scans (cardiac 4D-CT) were made on 15 pigs with a multidetector 64 row Siemens Somatom Definition Flash scanner (Siemens Healthcare, Forchheim, Germany) with $1 \mathrm{~mm}$ voxel and $1 \mathrm{~mm}$ slice spacing. There was no breathing motion present, since a breath-hold technique was used. Cardiac motion was based on electrocardiography (ECG) and was divided into 10 sequential states (0-9). Eight pigs had a pacemaker implanted, because the irradiation was planned to damage the atrioventricular (AV) node and a pacemaker should compensate for that. Pigs are therefore divided into two groups, with pacemaker (PM), $n=8$, and without one (noPM), $n=7$. The $4 \mathrm{D}$-CT were acquired between $2^{\text {nd }}$ and $16^{\text {th }}$ July and the irradiation took place between $21^{\text {st }}$ and $24^{\text {th }}$ July.

After the CT acquisition, DIR on cardiac 4D-CT was calculated using the B-Spline Plastimatch module in Slicer (see Section 2.2.2). Details on parameters used for DIR can be found in Table 2.3. State 0 was chosen as a reference state. State 3 corresponds to a maximum heart contraction with likely the biggest motion. All other states were registered to the the reference state with an inverse registration as well. A checklist was made to follow DIR and DIRQA for quality assurance. An example of a filled-out checklist is shown in Fig. 2.12a. 
Based on lung patient DIR and because of the time constraints in the study workflow, DIRQA was made only on DIR from state 5. DIRQA consisted of default and true absolute differences, true Jacobian and ICE. DIRQA results were stored in a text file (example shown in Fig. 2.12b) and users checked if the values did not exceed expected ones: Mean absolute difference should be smaller than 1; mean Jacobian should be 1 ; mean ICE should be smaller than $2 \mathrm{~mm}$. A box-shaped ROI was created in Slicer to encompass the pig body and then used in all DIRQA checks.

After a successful DIR and DIRQA, vector fields were used for treatment planning and the resulting plans were used in the pig irradiation experiment.

Around 20 minutes were needed for each pig DIR and additional 20 minutes for pig DIRQA. Calculations were done on a cluster of Linux computers, each with 8 CPU cores and 32 GB RAM.

Table 2.3.: Parameters used for Plastimatch registration. A mean squared error metric was used. Details for each parameter can be found here [Plastimatch, 2016].

\begin{tabular}{|c|c|c|}
\hline \hline Parameter & Stage 1 & Stage 2 \\
\hline Resolution & $4,4,2$ & $2,2,1$ \\
Grid size & 50 & 15 \\
Regularization lambda & 0.005 & 0.005 \\
Iterations & 200 & 100 \\
\hline \hline
\end{tabular}

\section{Post-experiment analysis}

After the completion of the animal study, a more detailed DIRQA was made, with all motion states included in DIRQA. In addition to the original checks explained in the previous section, vector field magnitudes were analyzed, the inverse absolute difference and the Jacobian were calculated. Paired t-tests were used to test statistical significance for vector field magnitudes between the true and inverse vector fields and PM and noPM groups. For linear fit quality estimation, a Pearson's r coefficient was used. 
(a)

Checkliste Bestrahlungsplannung

DOSE: YQOY TARGET: PV SCHWEIN: UnFOIM

Serlpts path: Axd/user/motion/Beamatme/CStAc7/simulations/Sckiprs

Heartbeats during CT: 4 bpm Finalmotion: Sin $3 \mathrm{~mm}$ - $1200 \mathrm{~ms} \mathrm{O}$

Task C.53 Script Done? Name Date

\begin{tabular}{|c|c|c|c|c|}
\hline Create Header & & $\sqrt{ }$ & AE & $17 \cdot 07$ \\
\hline Copy CT data to PatientData2 & & V & AE & 17.01 \\
\hline Sort CTs (contrast/native) & & v & AE & 11 \\
\hline Sort motion phased & & $v$ & $A E$ & n \\
\hline Check \# of files in each phase & CTX.sh & $v$ & AE & 11 \\
\hline Check contrast and native slices & 11 & $V$ & $A E$ & 11 \\
\hline Create CTX & $n$ & 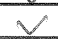 & $A E$ & 16 \\
\hline Create MHA & 11 & $\checkmark$ & $A E$ & ic \\
\hline Check BB coordinates and write Header & & V & $A E$ & II \\
\hline Copy Contours & & $\checkmark$ & $A E$ & 1802 \\
\hline Create VDX & & $v$ & AE & a \\
\hline Change 0 to 1 for Target (VDX) & & V & $A L$ & 8 \\
\hline Check voi names in VDX & & $\mathrm{V}$ & $\mathrm{AE}$ & $u$ \\
\hline
\end{tabular}

\begin{tabular}{|l|l|l|l|l|}
\hline FalseColorCheck contrast $\rightarrow$ snative $(00)$ & $\mathbb{A E}$ & $A .07$ \\
\hline Contours native ok? & & & & \\
\hline Registration contrast $\rightarrow 40 C T$ & & $\checkmark$ & $A E$ & 17.07 \\
\hline Registration ok? & & $\checkmark$ & $A E$ & 17.07 \\
\hline
\end{tabular}

(b)

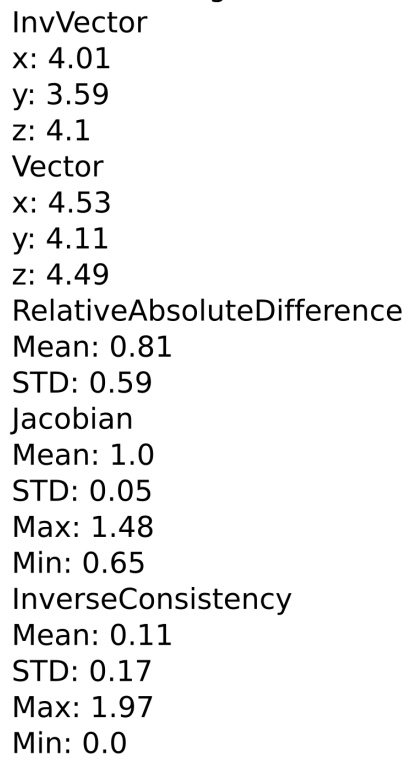
InvVector

$x: 4.01$

y: 3.59

z: 4.1

Vector

$x: 4.53$

$y: 4.11$

z: 4.49

RelativeAbsoluteDifference

Mean: 0.81

STD: 0.59

Jacobian

Mean: 1.0

STD: 0.05

Max: 1.48

Min: 0.65

InverseConsistency

Mean: 0.11

STD: 0.17

Max: 1.97

Min: 0.0

DIRQA for: Registration Node Contrast 4D

Figure 2.12.: (a) A part of the checklist for quality assurance during the pig irradiation. DIR and DIRQA part is highlighted in red and consisted of two steps. First, DIR was made on a cardiac 4D-CT and afterwards DIRQA was made on DIR from state $50 \%$. The final result was presented as text shown in (b). The relative absolute difference stands for ratio between the true and default absolute difference. 

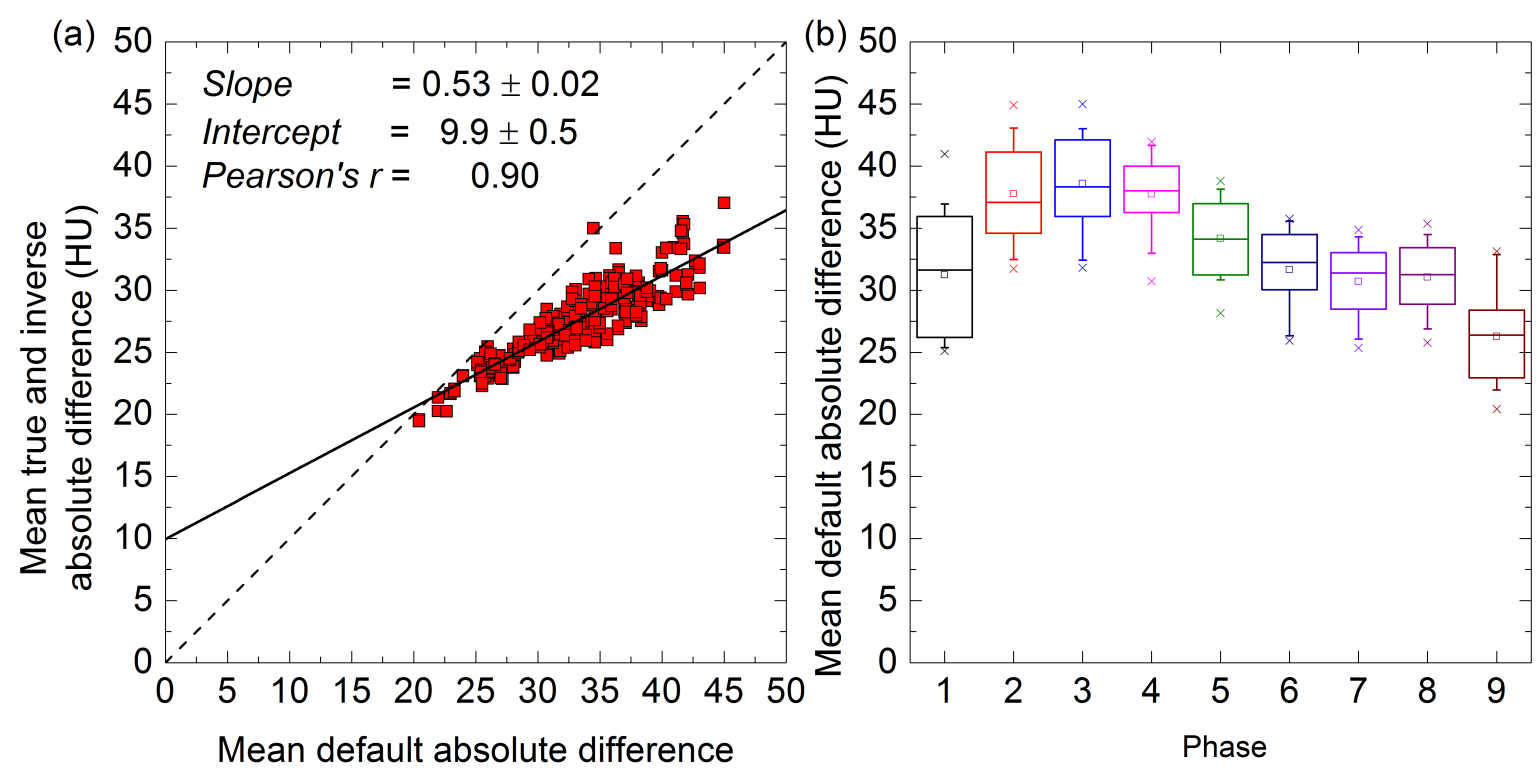

Figure 2.13.: (a) Mean true and inverse absolute difference plotted against mean default absolute difference. The solid line shows a linear fit, with parameters written in the top left corner. Dashed line shows $y(x)=x$. (b) Box plots of mean default absolute difference distribution across nine 4D-CT states. Boxes represent $25-75 \%$, whiskers $10-90 \%$ of data. The median is shown with a solid line, the mean is represented with squares and outliers with crosses.

\section{Results}

An example of a pig cardiac 4D-CT DIR is shown in Fig. 2.14. One DIRQA during the animal study showed higher mean true absolute difference than mean default absolute difference. The registration was therefore repeated with three stages instead of 2 . The third stage had 100 iterations with resolution size "1, 1, 1" and grid size "10". All other DIRQA checks were positive.

A post-experiment statistical analysis on vector field magnitudes is shown in Table 2.4. No statistical difference was observed between the true and the inverse vector fields. However, significant differences were observed between the vector field magnitudes of PM and noPM groups. The contributions to vector field magnitudes from three axis were equal.

Table 2.4.: Data for vector magnitudes. Values are presented as mean (range).

\begin{tabular}{|c|c|c|c|c|}
\hline \hline & \multicolumn{2}{|c|}{ PM } & \multicolumn{2}{c|}{ noPM } \\
\hline & True vector field & Inverse vector field & True vector field & Inverse vector field \\
\hline Mean & $0.08(0.03-0.16)$ & $0.08(0.03-0.14)$ & $0.07(0.0-0.18)$ & $0.06(0.0-0.17)$ \\
STD & $0.4(0.09-0.78)$ & $0.36(0.08-0.68)$ & $0.3(0.05-0.77)$ & $0.28(0.04-0.71)$ \\
Max & $8.24(1.6-17.33)$ & $7.98(0.7-17.76)$ & $5.9(0.97-15.91)$ & $5.38(1.08-12.42)$ \\
\hline \hline
\end{tabular}



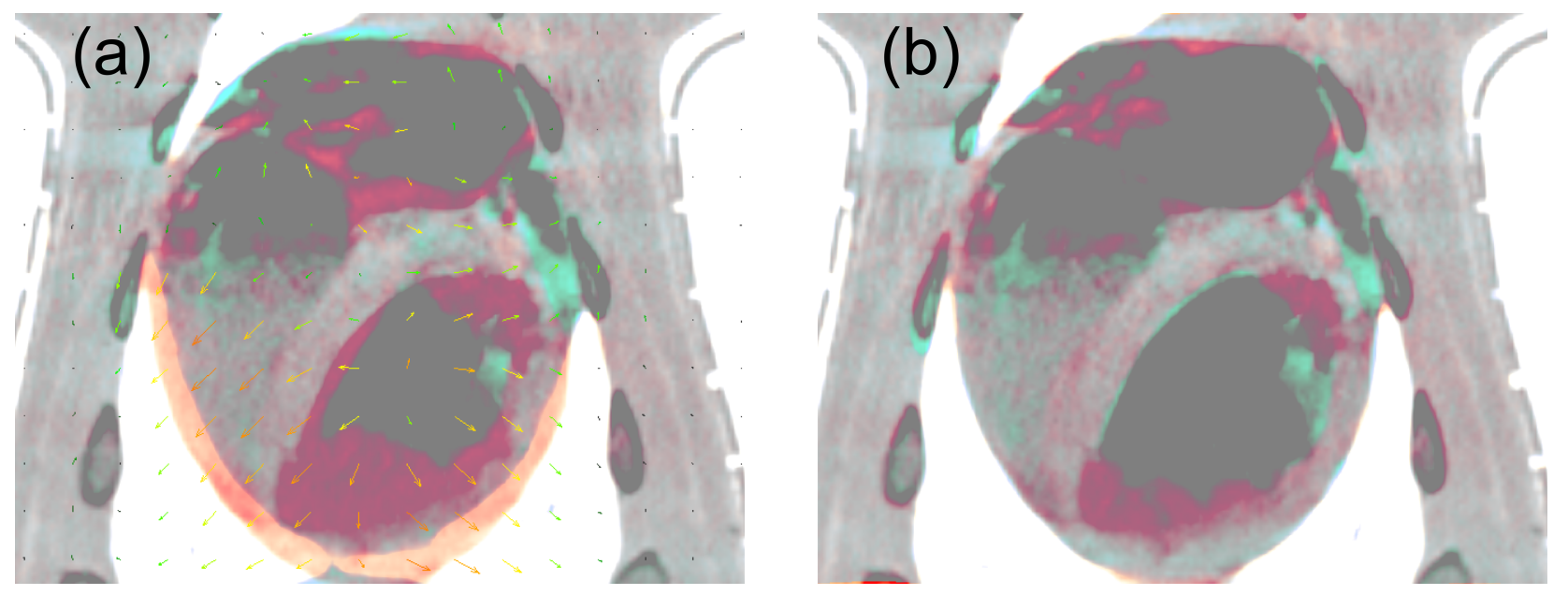

Figure 2.14.: An inverse color overlay of two states before (a) and after (b) DIR. The vector field is displayed on image (a) as arrows.

The dependence of the true and inverse absolute difference on default absolute difference with a linear fit is shown in Fig. 2.13a. The default absolute difference distribution across 9 states can be seen in Fig. 2.13b.

The distribution of the Jacobian and ICE results are shown in Fig. 2.15. Maximum values of true and inverse Jacobian and maximum ICE were tested against maximum vector magnitudes and fitted with a linear function. Results are plotted in Fig. 2.16.

All linear fits in Fig. 2.13 and 2.16 were statistically significant $(\mathrm{p}<0.05)$. 

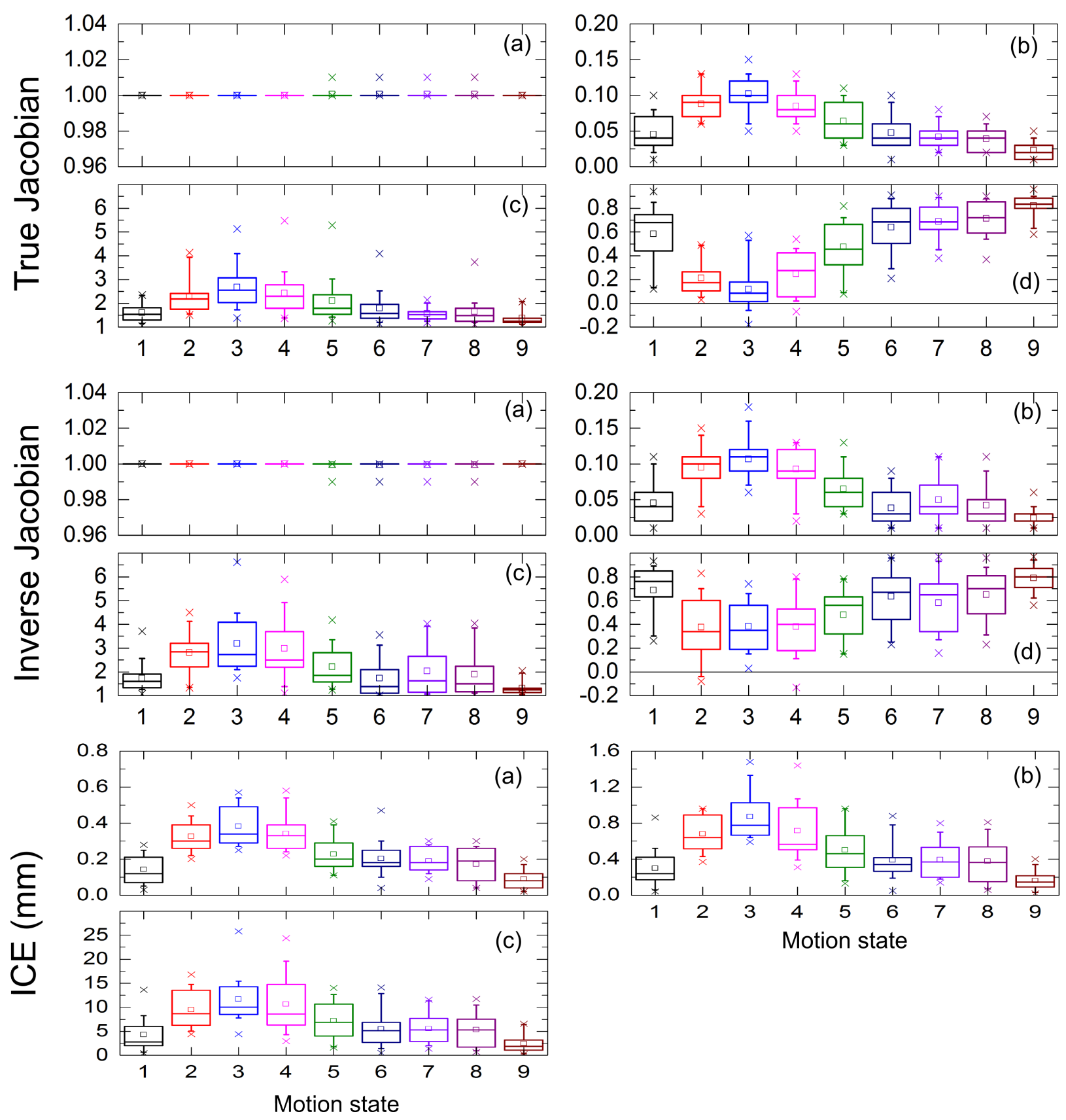

Figure 2.15.: Statistical data for true (top) and inverse (middle) Jacobian and ICE (bottom) for 9 cardiac 4D-CT states (reference state 0 is excluded) for 15 pigs. Mean, STD, maximum and minimum are represented as (a), (b), (c) and (d), respectively. Minimum ICE is 0 throughout all states and pigs. Boxes represent $25-75 \%$, whiskers $10-90 \%$ of data. The median is shown with a solid line, the mean is represented with squares and outliers with crosses. 


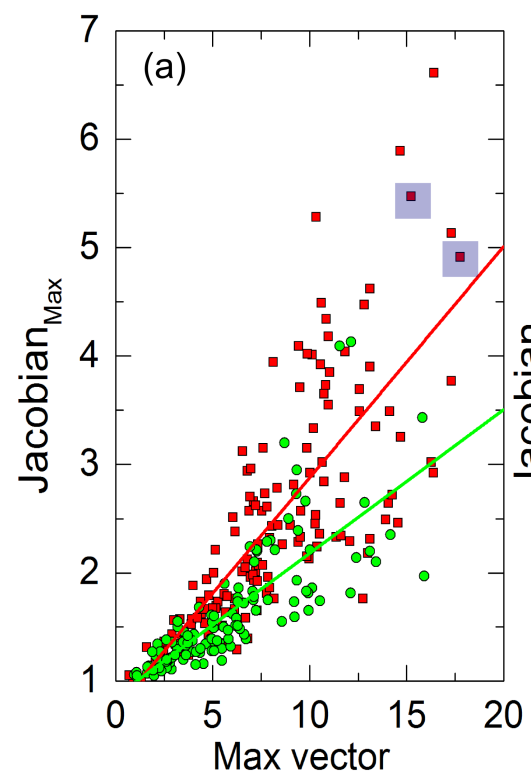

field magnitude $(\mathrm{mm})$

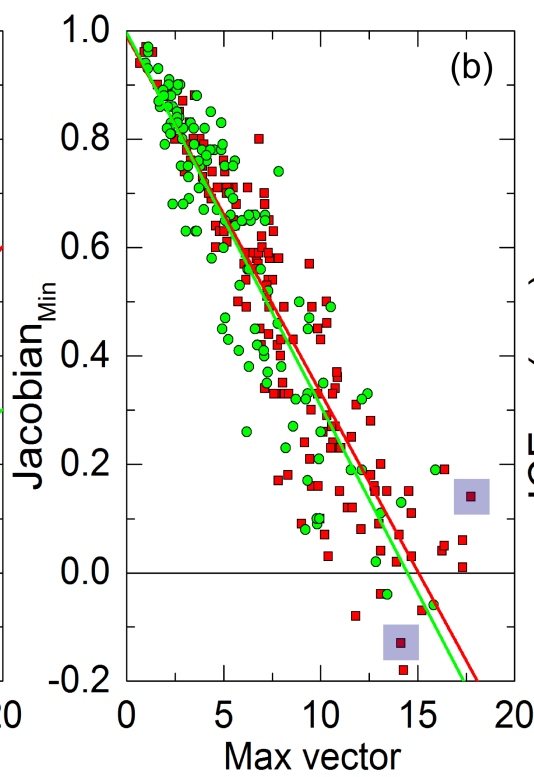

field magnitude $(\mathrm{mm})$

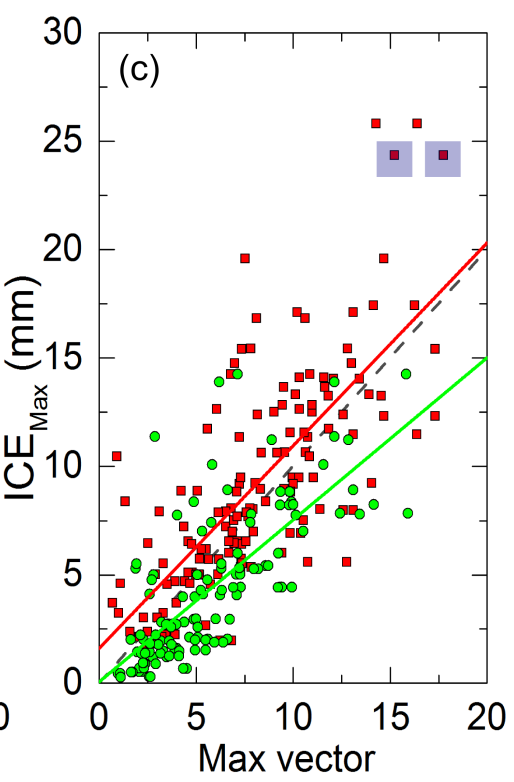

field magnitude $(\mathrm{mm})$

\begin{tabular}{lcccccc}
\hline & \multicolumn{2}{c}{ Jacobian $_{\text {Max }}$} & \multicolumn{2}{c}{ Jacobian $_{\text {Min }}$} & \multicolumn{2}{c}{ ICE $_{\text {Max }}$} \\
& PM & noPM & PM & noPM & PM & noPM \\
Slope & $=0.21 \pm 0.01$ & $0.13 \pm 0.01$ & $-0.07 \pm 0.002$ & $-0.07 \pm 0.002$ & $0.94 \pm 0.07$ & $0.77 \pm 0.06$ \\
Intercept & $=0.74 \pm 0.13$ & $0.86 \pm 0.06$ & $0.99 \pm 0.02$ & $1.0 \pm 0.02$ & $1.60 \pm 0.65$ & $-0.15 \pm 0.41$ \\
Pearson's $r=$ & 0.77 & 0.78 & -0.91 & -0.90 & 0.73 & 0.74
\end{tabular}

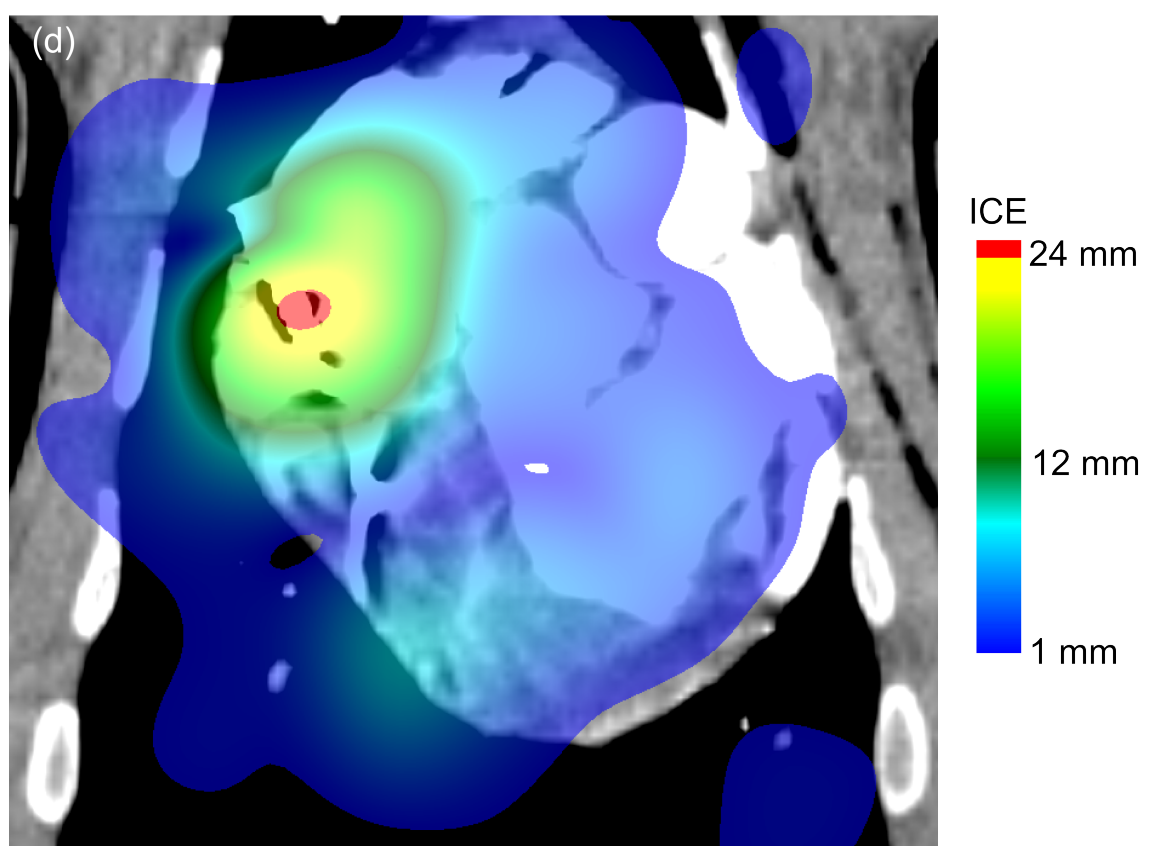

Figure 2.16.: Values of maximum Jacobian (a), minimum Jacobian (b) and maximum ICE (c) plotted against maximum vector magnitudes. A linear fit is displayed with a solid line and its parameters are written below the plots. The dashed line in (c) shows $y(x)=x$ plot. Values from cardiac 4D-CT state on image (d) are highlighted with blue squares in (a)-(c). PM and noPM are shown with red squares and green circles, respectively. ICE is displayed on (d) using the color table as displayed in the legend. 


\section{Discussion}

All 15 pig 4D-CTs have been registered, which resulted in 270 DIRs. DIRQA was performed in two steps - a smaller DIRQA during animal study on one state and a complete DIRQA on all 4D-CT states afterwards.

Mean vector field magnitudes were small (approx. $0.1 \mathrm{~mm}$ ), since pigs were in a breath-hold position and the only motion was the heartbeat. Despite the small mean vector magnitudes it was still enough to observe statistical difference between PM and noPM. Consequently, the difference between the two groups is consistent throughout the DIRQA.

DIR did well in terms of lowering the absolute difference. There was a strong correlation between default versus true and inverse absolute difference. The slope of the linear fit on Fig. 2.13 has the same value than the slope from lung 4D-CT (see Fig. 2.8), showing the effectiveness of the B-Spline algorithm. The distribution of the default absolute difference across different states is smaller than in lung 4D-CT (10 HU compared to $35 \mathrm{HU}$ ), due to the fact less motion was present in a pig cardiac 4D-CT. The shape of default absolute difference distribution persist then in the Jacobian and ICE distributions as well.

A good result in absolute difference does not necessary mean a good DIR, as can be seen from Jacobian and ICE checks. The mean Jacobian and ICE were 1 and 0 , respectively, since the vector fields were small on average. However there were large deviations present in Jacobian and ICE. Most notably, there were a few cases of negative minimum Jacobian which would suggest organ folding. Since organ folding does not occur during a heart beat, negative minimum Jacobian points to inconsistencies in DIR.

The large deviations in Jacobian and ICE can in part be explained with large maximum vector field values, as shown in Fig. 2.16. All linear fits have a good correlation, with no difference between PM and noPM in the quality of the fits. The actual linear fit parameters, however, further show the inconsistencies in DIR. The clearest example of inconsistencies in DIR is with a linear fit from maximum ICE PM, which lies above the function $y(x)=x$. This means that there were points further away from the starting point after the true and inverse transformation, then just after true transformation. The linear fit for noPM maximum ICE showed better results in this terms, since it lied below the $y(x)=x$ function.

During the animal study only one DIR was repeated because of DIRQA. It was shown in postexperiment analysis, that most of the DIRs should be repeated, pointing out flaws in initial DIRQA procedure. Mainly, DIRQA should be made on all DIR and not just on one state, since DIRQA from one DIR does not guarantee the quality of the other DIRs from the same 4D-CT. Each DIR is performed individually and should be treated as such. Furthermore, instead of mean Jacobian and ICE, maximum and minimum should be investigated, because it points to the worst part of the DIR. 


\subsection{Summary and Discussion}

Tools to perform DIR and DIRQA have been presented in this chapter. Modules were written for an open-source software Slicer that can handle large DIR problems, such as registering whole 4D-CTs. In addition to DIR, the modules can also provide quantitative information on DIR quality. The main objective of this work was to provide a systematic approach for DIR and to give parameters on DIRQA that can estimate the quality of DIR. A first analysis of DIRQA checks was done on a large DIR database - 684 DIR were checked in total.

Most of the work was based on Slicer, which is a well-established software in medical research. To date, there are more than 500 publications that have used Slicer in their research [Slicer, 2016b], with topic ranging from teaching [Pujol et al., 2016], disease staging [Liu et al., 2015, Liu et al., 2016a], motion tracking [Behringer et al., 2015] to image reconstruction [Meyer et al., 2015], image registration [Li et al., 2015b, Fedorov et al., 2015, Li et al., 2015a] and others. Slicer offers ample functionalities and is especially suited for research, since it can be modified to specific needs. However, it is important to stress that Slicer is not a medical product and as such can not be used in clinic. Additionally, Slicer can sometimes be unstable with unexpected crashes. It is constantly under development and more and more errors are fixed with each new release. New releases also bring new functionalities, but there can be problems with backtrack compatibility.

Results shown in this chapter were obtained with the B-Spline DIR algorithm. Several other algorithms exist, demons most commonly used alongside B-Spline [Thirion, 1998]. Varadhan et al. compared B-Spline and demons DIR for lung cases [Varadhan et al., 2013] and showed that B-Spline is superior to demons, especially if there is a difference in contrast between the images. They used a mutual information metric, to account for differences in contrast. Images used in this chapter were either all without (lung 4D-CTs) or all with (animal study 4D-CTs) contrast agent, therefore no difference in contrast between images was present and mutual metric could not be used.

A designated module was written for DIRQA. The main advantage of the DIRQA module is that all different techniques are gathered in a single place and can be used on a specific case. The ease of use is also essential, for DIRQA to find its way into clinical work flow. A test of using DIRQA in potential clinical work flow was done at GSI during the animal study, where different users operated with both DIR and DIRQA modules. The experiment was carried out under time pressure, since there was a scheduled beam time. 4D-CTs were acquired approximately two weeks before the scheduled irradiation. During this two weeks contour delineation, DIR, DIRQA, treatment planing and treatment planning QA had to be done [Graeff et al., 2015]. There were already propositions for frameworks for DIRQA in clinical work flow [Varadhan et al., 2013], however none were tested in an actual clinical environment. An animal study can not be directly compared to an actual clinic, however the number of pigs studied was high (15) and 
the study was under time constraint, which simulate some of the pressures present in clinical environment.

The techniques used in DIRQA were divided into qualitative (inverse color, checkerboard) and quantitative (absolute difference, Jacobian and ICE). While the quantitative can be used to pinpoint errors in DIR, the qualitative can be used to actually locate the error as shown in Fig. 2.9d. The location and size of DIR error also determines if a repetition of DIR is necessary. All three quantitative checks have been used in literature as a possible DIRQA [Varadhan et al., 2013, Leow et al., 2007, Christensen and Johnson, 2001, Bender and Tomé, 2009]. They all share the same flaw, however, that they are a necessary but not sufficient condition for a successful DIR. One common DIRQA check in literature that our module is currently missing, is comparison of anatomical correspondence - comparison between reference, moving and warped contours. Ideally the warped and the reference contour should be the same. Two metrics are usually used in contour comparison - dice similarity coefficient [Varadhan et al., 2013] and Hausdorff distance [Huttenlocher et al., 1993]. Slicer already has functionalities for both contour comparison checks, so they could be used. The biggest disadvantage of the anatomical correspondence check is that the contour delineation is required in both, the reference and moving phase, which is scarcely done by physicians, since it takes too much time. Additionally, the anatomical correspondence check does not judge the vector field quality inside contour. The lack of contours in both reference and moving phase was the reason the anatomical correspondence check was not used.

Studies on DIRQA so far have focused on a small number of DIR cases, whether it is phantom [Mutic et al., 2001, Moore et al., 2004] or patient studies [Wu et al., 2008, Varadhan et al., 2013]. With a small number of DIRs, it is possible to thoroughly examine each DIR and hence understand DIRQA. In this work a different approach was used. Rather than examining each DIR individually, a large dataset was analyzed and common traits for DIR were found. Due to differences in anatomical sites, DIRQA parameters have to be found for each anatomical site individually, since they can deviate significantly, as seen by two different cases presented here. However, three checks are independent on anatomical site: mean true and absolute difference should be lower than default absolute difference, Jacobian should be positive and ICE should be smaller than maximum vector field magnitudes. If any of these checks fails, DIR needs to be investigated and, if necessary, repeated.

DIR of a lung 4D-CT can be considered relatively easy, since the contrast between lungs and other tissue is high. This is confirmed by a mean value of 1 for the Jacobian and mean ICE smaller than $1 \mathrm{~mm}$. The maximum and minimum Jacobian and ICE are more interesting, since they show DIR inconsistencies. All ICE values bigger than maximum vector field magnitudes were found to originate from areas with a poor DIR. An effect of a poor DIR can be seen in Fig. 2.11, where the propagated liver and lung contour do not match features on the image. An image artifact was the reason for the poor DIR. After investigation of poor DIR location and 
size, it was decided, that DIR does not require repetition, due to large distance between poor DIR and the tumor.

If the DIR of lung 4D-CT was considered relatively easy, opposite holds true for DIR of animal study 4D-CT. The motion of the heart during a heartbeat is complex, with muscles relaxing and contracting in different directions [Seeley et al., 2007]. Furthermore, the volume of blood shifts from one ventricle to the other. In the case of a cardiac 4D-CT, blood carried a contrast agent and blood distribution in heart was changing during a heartbeat. Therefore the HU distribution varied drastically in different cardiac 4D-CT states. Additionally, it is well established that pacemakers cause several complications in a CT scan [Mak and Truong, 2012]. This was confirmed by the differences observed between PM and noPM. The clearest example is the PM linear fit of the maximum ICE in Fig. 2.16, which is above $y(x)=x$. For noPM the linear fit is below $y(x)=x$, however the slope has still a value of 0.77 , compared to 0.38 of a lung 4D-CT fit. Inconsistencies in DIR were further supported by negative minimum Jacobian, which were found for both, PM and noPM groups. Negative minimum Jacobian and large ICE values are clear indicators, that DIR in heart can not be accepted for heart treatment planning and needs to be repeated. An effect of DIR on actual irradiation also has to be examined. The DIR of cardiac 4D-CT is currently under careful investigation and several different solutions, such as artifact removal and different registration parameters are being tested.

In the future, the DIRQA module should undergo further testing. In addition to checking DIRQA on different anatomical sites and between different modalities, it should be investigated how good DIRQA is at spotting inconsistencies in DIR, i.e. what is the number of false negatives. Furthermore, with more data analyzed, the parameters in DIRQA checks should get more precise, so outliers could be more easily spotted.

Based on the findings presented here, several new features could improve the DIRQA module. Instead of the mean, STD, maximum and minimum values, histograms could be displayed for quantitative tests. Furthermore, histograms could be displayed for a specific contour, such as the tumor, which would give a direct indication of DIR quality effect on treatment planning. Additionally, the module should automatically show the location of maximum and minimum Jacobian and maximum ICE. The worst part of DIR could be than immediately recognized and appropriate response could be formed. 


\section{Comparison of Photons versus Carbon lons in Single Fraction Therapy of Lung Cancer}

\subsection{Introduction}

Lung cancer is one of the leading medical problems worldwide with approximately 1.4 million deaths per year [Siegel et al., 2014]. Surgery is usually the first choice in treating localized non-small cell lung cancer (NSCLC). However, in recent years stereotactic body-radiation therapy with photons (SBRT) showed very promising results, with high local control-rates of NSCLC [Baumann et al., 2009, Fakiris et al., 2009, Grutters et al., 2010, Ricardi et al., 2010, Timmerman et al., 2010, Greco et al., 2011].

Scanned particle therapy can produce sharp dose gradients with a finite range of the beam and can thus provide higher normal tissue sparing. This reduces both side effects as well as the risk of secondary cancer [Newhauser and Durante, 2011]. Treatment of lung tumors with particles is still challenging due to interplay and radiological path length changes [Bert and Durante, 2011]. The latter can be substantial when dense tissue (e.g. the solid tumor mass) is replaced with low-density tissue (lung) due to motion.

Grutters et al. have performed a meta-analysis on comparison between photon, proton and carbon ions in treating NSCLC [Grutters et al., 2010]. They found similar 5-year survival rates for SBRT, protons and carbon-ions (around 40\%). However, the number of patients treated with particle therapy was low and they advise caution when interpreting the data. Also different fractionation schemes were used in the comparison. A more recent review was published by Kamada et al. [Kamada et al., 2016] where they reported a high 3-year survival rate for singlefraction carbon-ions (76.9\%), with no late treatment-related adverse effects. In comparison, SBRT had 55.8\% 3-year survival rate, with $10-27 \%$ of patients exhibiting grade 3 treatmentrelated adverse effects [Timmerman et al., 2010]. It is important to note that all of these studies used passive beam scattering, avoiding the problem of interplay between organ motion and scanning beam motion. On the other hand, active beam scanning can provide even better dose shaping which becomes essential in high dose single fractionation regimes. The effects of motion and motion mitigation techniques on scanned carbon ion dose distribution therefore need to be considered in a fair comparison of photons and carbon ions. 
To evaluate potential advantages of active scanning with carbon ions (PT), an in silico comparison of simulated PT plans to SBRT plans actually delivered was conducted. Target coverage and a wide range of OAR doses were assessed both with and without simulated motion on time-resolved computed tomographies (4D-CTs).

\subsection{Materials and methods}

\subsubsection{Patient data}

The study included 19 patients with in total 26 lesions that were actually treated with SBRT at the Champalimaud Centre for the Unknown, Lisbon (Portugal). The lesion size was $2.9 \mathrm{~cm}^{3}$ (median, 25-75\% 1.4 - 9.7) and peak-to-peak motion was $3.1 \mathrm{~mm}$ (1.6 - 5.6). Three patients had two targets, one had five and the rest one. 13 lesions were right-sided, 12 were left-sided and one was located in right cardiophrenic space. An overview of tumor characteristics can be found in Table 3.1. Two CTs were available for all patients. A planning CT was used for OAR delineation and SBRT planning. Target motion was estimated on a 4D-CT, consisting of 10 phases (0\% - 90\%). Clinical target volumes (CTV) were delineated using a registered positron emission tomography (PET) scan.

The planning objectives were that $99 \%$ of planning target volume (PTV) must receive at least $24 \mathrm{~Gy}\left(D_{99 \%} \geq 24 \mathrm{~Gy}\right)$ in a single fraction, while all OAR constraints as defined in the AAPM task group 101 report on stereotactic radiotherapy had to be respected [Benedict et al., 2010]. Details on OAR constraints can be found in Table B.1.

\subsubsection{Planning target volume definition}

To account for range changes relevant for particles only, different PTV definitions were used for SBRT and PT, as shown in Fig. 3.1. Within this chapter they will be named PTV $\mathrm{SBRT}_{\text {and }} \mathrm{PTV}_{P T}$ for SBRT and PT, respectively. In SBRT, the responsible clinician determined the maximum breathing motion of the CTV from the 4D-CT, hence creating an ITV. This ITV plus an additional $3 \mathrm{~mm}$ for setup uncertainty yielded the PTV $\mathrm{PTRT}_{S B}$.

$\mathrm{PTV}_{P T}$ was constructed following principles from Graeff et al [Graeff et al., 2012]. Each beam has a unique $\mathrm{PTV}_{P T}$. For setup uncertainty margins of $3 \mathrm{~mm}$ laterally and $1 \mathrm{~mm}$ in beam's eye view (BEV) were used on the CTV. Afterwards a water-equivalent path length ITV (WEPL-ITV) was build, using transformation maps from the B-Spline deformable registration of the 4DCT data [Shackleford et al., 2010]. Additional $2 \mathrm{~mm}+2 \%$ proximal and distal margins were added in BEV to account for uncertainty from Hounsfield units to water equivalent path length conversion. If the target overlapped with an OAR (e.g. small airways) then OAR plus a margin of 2-5 mm was subtracted from PTV $\mathrm{PBRT}_{\text {or }} \mathrm{PTV}_{P T}$. 
Table 3.1.: Lesion characteristics, with lesion locations, stages, peak-to-peak motions and volumes of corresponding CTV, PTV SBRT $_{\text {and }}$ PTV $_{P T}$. Abberevations for lesion location are: $R S L$, right superior lung; IRL, inferior right lung; $L S L$, left superior lung; ILL, inferior left lung; RCS, right cardiophrenic space.

\begin{tabular}{|c|c|c|c|c|c|c|c|}
\hline \multirow[b]{2}{*}{$\begin{array}{l}\text { Patient } \\
\text { Number }\end{array}$} & \multirow[b]{2}{*}{$\begin{array}{c}\text { Lesion } \\
\text { Number }\end{array}$} & \multirow[b]{2}{*}{ Location } & \multirow[b]{2}{*}{ Stage } & \multirow[b]{2}{*}{$\begin{array}{l}\text { Peak-to-peak } \\
\text { motion }[\mathrm{mm}]\end{array}$} & \multicolumn{3}{|c|}{ Volume $\left(\mathrm{cm}^{3}\right)$} \\
\hline & & & & & CTV & $\mathrm{PTV}_{S B R T}$ & $\mathrm{PTV}_{P T}$ \\
\hline 1 & 1 & LSL & IIa & 4.8 & 35.9 & 100 & 179 \\
\hline 2 & 2 & LSL & Ia & 3.1 & 1.6 & 7.7 & 40.6 \\
\hline 3 & 3 & IRL & IV & 12 & 2.3 & 11.6 & 32 \\
\hline 3 & 4 & RSL & Ia & 0.5 & 6.9 & 25.2 & 38 \\
\hline 4 & 5 & ILL & IV & 4.4 & 2.5 & 15 & 20.5 \\
\hline 5 & 6 & ILL & IV & 7.5 & 1.4 & 7.7 & 26.5 \\
\hline 6 & 7 & RSL & IV & 3.9 & 16 & 40 & 72.5 \\
\hline 7 & 8 & ILL & IV & 0.6 & 139 & 261 & 255 \\
\hline 8 & 9 & LSL & IV & 2 & 9.2 & 35 & 46.5 \\
\hline 8 & 10 & IRL & IV & 3.4 & 10.2 & 38 & 45.5 \\
\hline 9 & 11 & ILL & IV & 2.8 & 14.4 & 46.4 & 57.2 \\
\hline 9 & 12 & ILL & IV & 5.8 & 3.8 & 17.4 & 23.4 \\
\hline 10 & 13 & RSL & IV & 0.8 & 4.3 & 17.7 & 26.3 \\
\hline 10 & 14 & LSL & IV & 3.4 & 2.7 & 14.5 & 23.1 \\
\hline 10 & 15 & RSL & IV & 2.1 & 3.1 & 15.4 & 33.5 \\
\hline 10 & 16 & LSL & IV & 0.5 & 0.5 & 5.4 & 6.7 \\
\hline 10 & 17 & ILL & IV & 7.8 & 0.8 & 6.1 & 23.5 \\
\hline 11 & 18 & LSL & IV & 0.1 & 1.7 & 15 & 23.5 \\
\hline 12 & 19 & IRL & IIIb & 11.4 & 27 & 137 & 118.5 \\
\hline 13 & 20 & RSL & Ia & 2.2 & 1.7 & 10 & 23.4 \\
\hline 14 & 21 & RSL & IV & 0.2 & 0.9 & 3.2 & 14.9 \\
\hline 15 & 22 & RSL & IV & 2.2 & 3.9 & 22.1 & 27.5 \\
\hline 16 & 23 & LSL & IV & 3.1 & 9.8 & 28 & 51 \\
\hline 17 & 24 & RSL & IV & 8.1 & 0.6 & 3.3 & 4.1 \\
\hline 18 & 25 & LSL & IV & 1.4 & 0.8 & 5.9 & 10 \\
\hline 19 & 26 & RCS & IV & 11.8 & 0.4 & 6.6 & 8.6 \\
\hline
\end{tabular}



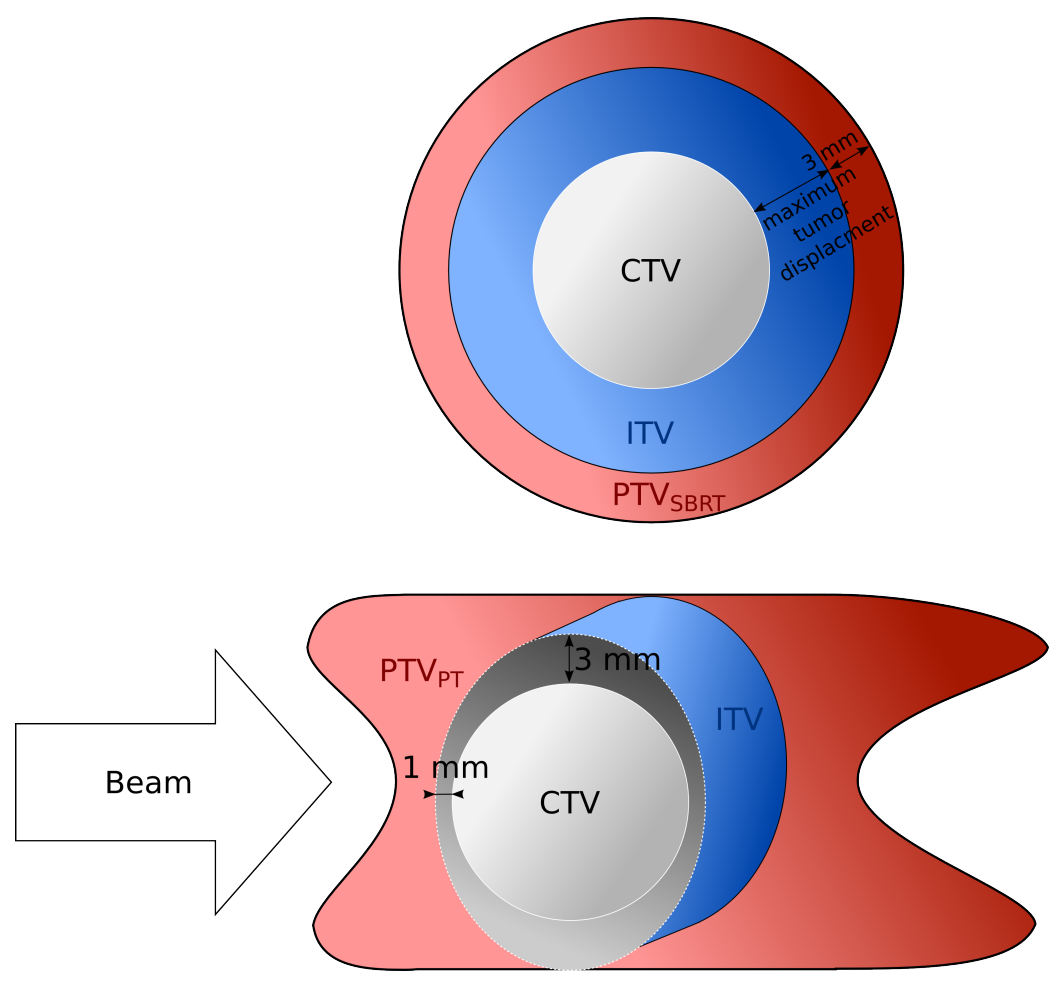

Figure 3.1.: Different PTV definitions for SDRT $\left(\right.$ PTV $\left._{S B R T}\right)$ and CiT $\left(\right.$ PTV $\left._{P T}\right)$. For PTV SBRT $_{\text {isotropic }}$ margins of $3 \mathrm{~mm}$ plus maximum tumor displacement due to breathing were used on the CTV; For PTV ${ }_{P T}$ margins of $3 \mathrm{~mm}$ laterally and $1 \mathrm{~mm}$ in beam's eye view were used and then range-ITV was constructed with $2 \mathrm{~mm}+2 \%$ range margins added for $\mathrm{PTV}_{P T}$ in end-inhale phase.

\subsubsection{SDRT treatment planning}

The clinical SBRT plans were calculated with the Eclipse v10 planning system (Varian Medical Systems, Palo Alto, USA) using the AAA beam model. They were all VMAT plans generally consisting of 4 overlapping partial arcs, 2 in clockwise and 2 anticlockwise direction, with a gantry range of typically $200^{\circ}$. For tumor sizes $>2.5 \mathrm{~cm}$ a calculation grid of $2.5 \mathrm{~mm}$ was used, otherwise this was $1 \mathrm{~mm}$. During optimization, a first iteration included the PTV $\mathrm{PBRT}_{\text {SB }}$ only, after which the OARs were added. In order to lower OAR dose and improve the PTV $\mathrm{TVRT}_{\text {SBT }}$ homogeneity, we created an artificial shell of $2 \mathrm{~cm}$ around the PTV $\mathrm{PBRT}_{\text {SR }}$ and minimized the dose there as well. During optimization the fast Multi Resolution Dose Calculation (MRDC) model was used, with one intermediate step using the slower but more accurate AAA model to get an adequate $\mathrm{PTV}_{\text {SBRT }}$ coverage after optimization. 


\subsubsection{Carbon-ion treatment planning}

For PT, state of the art 4D treatment planning software TRiP98 was used [Richter et al., 2013]. A single field uniform dose plan (SFUD) was optimized on the $\mathrm{PTV}_{P T}$ in the end-inhale reference phase of the $4 \mathrm{D}$-CT. Most targets $(n=20)$ were planned with two fields. For the remaining targets, one $(n=1)$, three $(n=3)$ or four $(n=2)$ fields were used due to proximity of OARs. A regular grid of beam spots with a spacing of $2 \mathrm{~mm}$, a beam spot full width at half maximum (FWHM) size of approximately $6 \mathrm{~mm}$ and a $3 \mathrm{~mm}$ ripple filter were used. To compensate for short particle ranges in lung tissue, a bolus of $80 \mathrm{~mm}$ water-equivalent thickness was added.

The relative biological effectiveness (RBE) following the local effect model (LEM) IV [Elsaesser et al., 2010]. For a conservative estimation, an alpha-beta ratio of 6 Gy and 2 Gy were used for target and OARs, respectively. This led to an RBE of approximately 1.1 in target tissue and approximately 1.1 to 3 in OARs. Dose was calculated on end-inhale (3D-Dose ${ }_{0 \%}$ ) and end-exhale (3D-Dose ${ }_{50 \%}$ ) phases. 4D dose delivery was simulated as described by Richter et al [Richter et al., 2014]. Two different breathing periods (3.6 and $5 \mathrm{~s}$ ) and two different starting phases $\left(0^{\circ}\right.$ and $\left.90^{\circ}\right)$ were used. Simulations without motion compensation (4D-Dose interplay ) and with slice-by-slice raster rescanning were performed (4D-Dose rescan $_{n}$ ). Five rescans were used for the majority of targets $(n=24)$, whereas 20 rescans were used for targets where the interplay effects were too big to achieve a satisfactory target coverage ( $n=2$; lesions 3 and 18 in Table 3.1).

\subsubsection{Dose metrics and analysis}

For comparison between SBRT and PT the following dose metrics were used - for the target the minimum dose in $99 \%$ of the volume $\left(D_{99 \%}\right)$, which should be higher than $24 \mathrm{~Gy}$; for OARs, the maximum point dose $\left(D_{\text {Max }}\right)$ and the mean dose $\left(D_{\text {Mean }}\right)$. Additionally, the volume receiving $20 \%$ of the planned dose $\left(V_{20 \%}\right)$ was used to assess differences in lung doses. In all cases, absorbed dose in Gy for SBRT was compared to biologically-equivalent dose in Gy(RBE) for PT.

Paired t-tests were performed to compare the dose metrics and for post-hoc exploratory analysis between groups a two-sided t-test with Welch correction for different variances was carried out. A p-value $<0.05$ was considered significant. Dose differences are always reported such that higher dose levels for SBRT result in positive values. 


\subsection{Results}

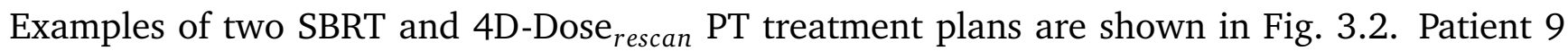
has two lesions in close proximity to the spinal cord. Patient 2 has a small lesion $\left(1.6 \mathrm{~cm}^{3}\right)$ in the superior position of the left lung. $D_{99 \%}$ is $100 \%$ for SBRT and PT in all CTVs for Patient A and B; average OAR difference between SBRT and PT in $D_{\text {Max }}$ is $5.1 \mathrm{~Gy}$ and $1.4 \mathrm{~Gy}$ and in mean dose $1.4 \mathrm{~Gy}$ and $0.7 \mathrm{~Gy}$, respectively for patient A and B.

\section{Patient 9}
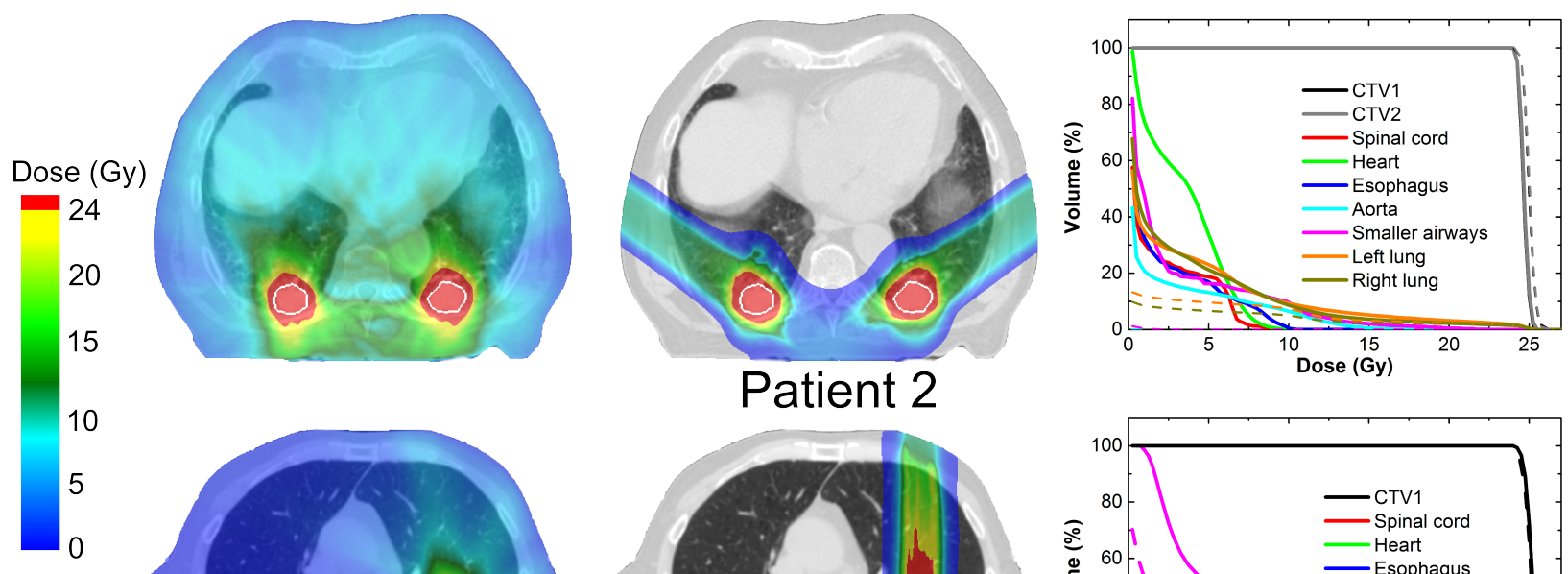

\section{Patient 2}
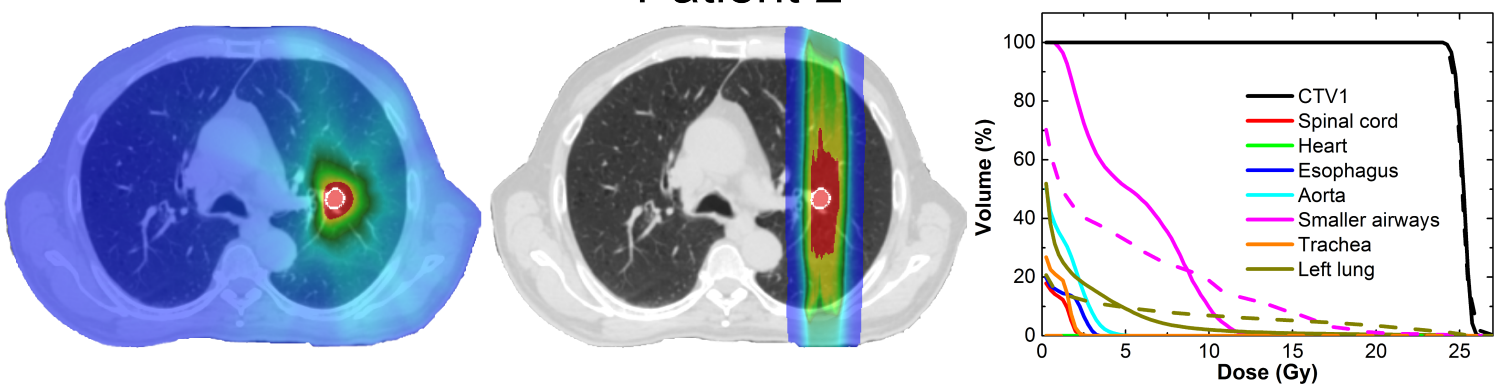

Figure 3.2.: Treatment plans for SBRT (left), PT (middle) and dose volume histogram (right) for SBRT (solid lines) and PT (dashed lines) for two patients. PT curves for OARs without any dose are not shown. Patient 9 (top row) might be better suited for PT and patient 2 (lower row) for SBRT. Patient 2 has a small lesion $\left(1.6 \mathrm{~cm}^{3}\right)$ in a central lung region, resulting in large $P T V_{P T}$ - up to $32 \mathrm{~cm}^{3}$, compared to PTV CTV contour is outlined in white.

\subsubsection{Target Coverage}

The difference in PTV definition resulted in 1.5 (1.3 - 2.1) times bigger PTV ${ }_{P T}$ than PTV SBRT $_{\text {. All }}$ SBRT plans were clinically acceptable, though in one case the $D_{99 \%}$ was reduced to 16.8 Gy due to proximity of an OAR. 3D-Dose $\mathrm{D}_{0 \%}$ and 3D-Dose ${ }_{50 \%}$ plans provided sufficient target coverage in all patients. For 4D-Dose $\mathrm{interplay}_{\text {and } 4 \mathrm{D} \text {-Dose }}$ rescan there was $63 \%$ and $2 \%$ cases of insufficient target coverage, respectively, across all targets and different breathing patterns. Details are shown in Fig. 3.3. For the patient with reduced dose in SBRT, PT could increase the $D_{99 \%}$ from $16.8 \mathrm{~Gy}$ in SBRT to $20.3 \mathrm{~Gy}$ while adhering to OAR constraints. 


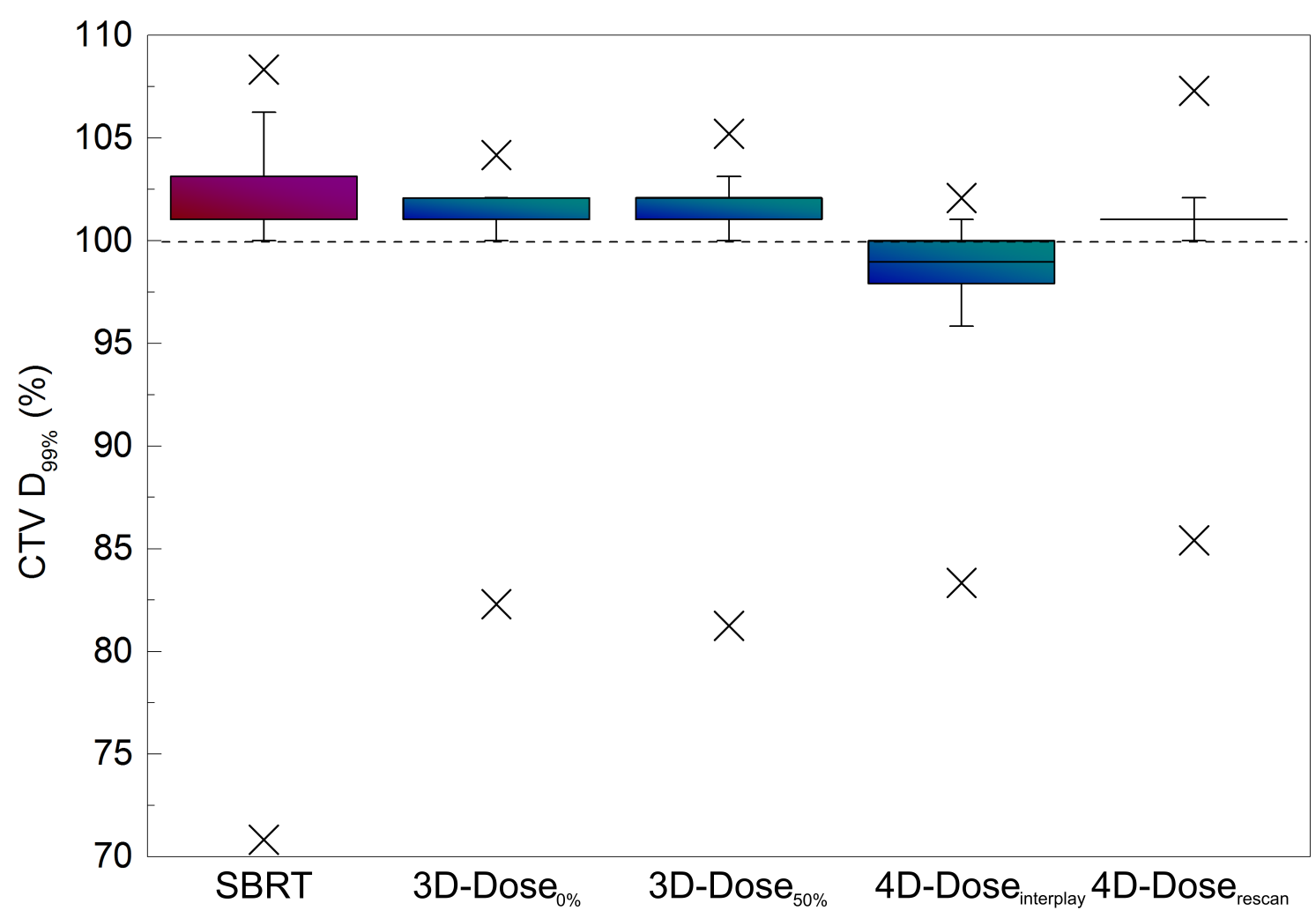

Figure 3.3.: CTV $D_{99 \%}$ for SBRT and different PT calculations. Four different breathing patterns are included for all targets in 4D-interplay and 4D-rescan. The dashed line shows the lower limit for clinical acceptance. One patient was an exception where lower target dose was accepted due to the proximity of a critical structure.

\subsubsection{Dose in OARs}

There was no significant difference in dose to OAR between the different PT dose calculations.

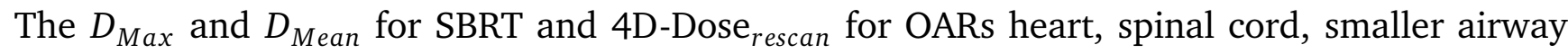
esophagus, trachea, aorta, ipsi- and contralateral lung are presented in Table 3.2.There was a significant difference in both $D_{\text {Max }}$ and $D_{\text {Mean }}$ for all OARs between SBRT and PT, with PT delivering less dose to OARs. Significant difference was also observed for $V_{20 \%}$ for ipsilateral lung, which was 15.3\% (9.6 - 23.5) and 10.3\% (7.9 - 13.7) for SBRT and PT, respectively. The $V_{20 \%}$ for contralateral lung was zero for almost all patients in SBRT and PT. The overall OAR difference for patients between SBRT and PT was significant, $2.8 \mathrm{~Gy}\left(1.6\right.$ - 3.7) for $D_{\text {Max }}$ and 0.7 Gy $(0.3-1.6)$ for $D_{\text {Mean }}$. 
Table 3.2.: Dose metrics for OARs. First value at each organ is from SDRT and the second from 4D-rescan. All values are shown as median and $25-75 \%$ in brackets.

\begin{tabular}{|l|c|c|c|c|}
\cline { 2 - 5 } \multicolumn{1}{c|}{} & \multicolumn{2}{c|}{$D_{\text {Max }}(\mathrm{Gy})$} & \multicolumn{2}{c|}{$D_{\text {Mean }}(\mathrm{Gy})$} \\
\hline OAR & Photon & Carbon & Photon & Carbon \\
\hline Heart & $6.0(0.3-11.6)$ & $0(0-8.8)$ & $1.3(0.1-2.2)$ & $0(0-0.5)$ \\
Spinal Cord & $5.5(3.3-8.5)$ & $0(0-0.5)$ & $0.7(0.3-1.2)$ & $0(0-0)$ \\
Smaller Airways & $13.0(9.8-17.1)$ & $10.3(3.3-19.1)$ & $2.8(1.5-5.8)$ & $0.5(0-2.6)$ \\
Esophagus & $5.8(3.9-8.4)$ & $0(0-0.3)$ & $1.1(0.6-1.5)$ & $0(0-0)$ \\
Trachea & $3.9(1.8-5.4)$ & $0(0-0)$ & $1(0.3-1.3)$ & $0(0-0)$ \\
Aorta & $8.0(5.1-21.9)$ & $3.9(0-18.1)$ & $1.4(0.7-1.6)$ & $0.1(0-0.4)$ \\
Ipsilateral Lung & $26.3(26.0-26.5)$ & $26.3(25.8-26.5)$ & $1.9(1.5-3.0)$ & $1.9(1.4-2.5)$ \\
Contralateral Lung & $5.0(3.5-9.6)$ & $0(0-0.9)$ & $0.4(0.2-0.6)$ & $0(0-0)$ \\
\hline \hline
\end{tabular}

\subsubsection{Dependence on CTV Size}

Significant differences were observed between patients with a single CTV smaller $(n=8)$ or larger $(n=7)$ than $2.5 \mathrm{~cm}^{3}$ for $D_{\text {Max }}$ and $D_{\text {Mean }}$, see Fig. 3.4. For patients with a smaller CTV, the dosimetric advantage over SBRT was on average 0.9 Gy and 0.5 Gy lower for $D_{\operatorname{Max}}$ and $D_{\text {Mean }}$, respectively. This was associated with PTV $_{P T}$ definition - the average volume ratio between PTV $_{P T}$ and PTV SBRT $_{\text {was }} 2.9$ (1.6 - 4.0) and 1.5 (1.3 - 1.8), for patients with CTV $<2.5$ $\mathrm{cm}^{3}$ and CTV $>2.5 \mathrm{~cm}^{3}$, respectively.

The 4 patients with multiple lesions were excluded from this comparison. The $D_{\text {Max }}$ and $D_{\text {Mean }}$ difference were on average higher in these patients, but the number of patients was too low for statistical analysis. 

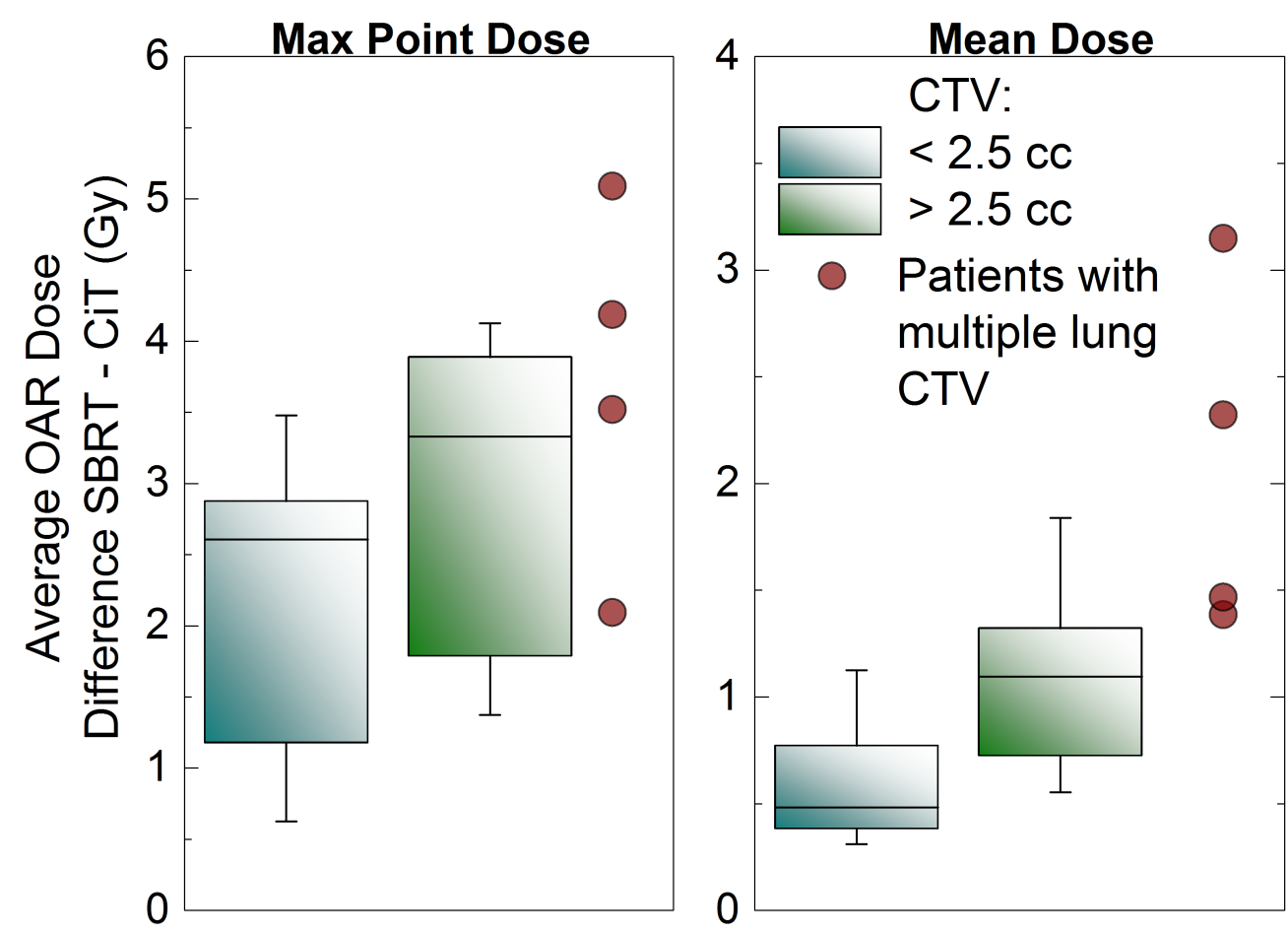

Figure 3.4.: Box plots of average OARs max point dose $\left(D_{\text {Max }}\right)$ and mean dose difference between SBRT and PT for patients with single CTV smaller $(n=9)$ or bigger $(n=6)$ than $2.5 \mathrm{~cm}^{3}$. Boxes represent $25 \%-75 \%$, outliers are shown as whiskers and median is shown with solid lines. Values for patients with multiple lesions are shown with circle symbols.

\subsection{Summary and Discussion}

This is the first in silico trial directly comparing clinically valid SBRT plans to scanned carbon ion plans using state of the art 4D dose calculation and motion mitigation methods for NSCLC patients. Our study found that PT deposited less dose to OARs compared to SBRT. Therefore PT might be considered as an alternative treatment option to SBRT. The finite range of the beam permits a small number of fields and thus a narrow entry channel, so that critical OARs such as spinal cord, heart, esophagus, and the contralateral lung could be effectively spared using PT, with typically low or even zero dose. PT could be thus highly beneficial to patients with impaired contralateral lung function, because PT deposited no dose in the contralateral lung in 12 patients, while SBRT irradiated the contralateral lung in all patients. Being an intensitymodulated arc therapy, SBRT had an advantage in some patients where the smaller airways were in a close proximity to CTV; SBRT could shape the dose distribution to reduce dose to the smaller airways, compensating PT's advantageous physical dose characteristics.

Further increase in OAR sparing could be achieved by using intensity modulated particle therapy (IMPT) instead of SFUD. While IMPT could lead to less dose in the OARs, it would 
make the plans less robust against setup errors due to additional dose gradients between the fields. These gradients can be controlled by employing robust optimization to account for range, motion and setup uncertainties, which we will implement in a future 4D treatment planning study [Chen et al., 2012, Graeff, 2014].

\subsubsection{Range Margins and Motion Mitigation}

Since conventional geometric margins are not suitable for PT [Park et al., 2012b], margins based on range changes were used. Another trial comparing photon to proton therapy in NSCLC patients also used different PTV definitions to incorporate range changes [Roelofs et al., 2012]. As shown in our study, inclusion of range changes leads to increase in $\operatorname{PTV}_{P T}$, up to 4.7 times compared to PTV ${ }_{S B R T}$. Furthermore, the difference between PTVs is bigger for smaller tumor sizes. Patients with bigger tumor volumes (CTV $>2.5 \mathrm{~cm}^{3}$ ) are therefore better suited for treatment with PT.

Our results confirm previously published results that interplay can lead to a dose degradation in treating moving targets with active scanned beam [Bert et al., 2008]. Fig. 3.3 shows the importance of using 4D dose calculation and motion mitigation techniques in treating moving targets with particles. Even small motion amplitude can lead to underdosage in CTV without proper motion mitigation. Considering the average over the 4 simulated motion patterns, 15 patients showed a $D_{99 \%}<24 \mathrm{~Gy}$ under interplay conditions, as opposed to none when using rescanning (excluding the one patient with reduced target dose). Rescanning proved to be a strong mitigation technique, with robust results across all targets and different breathing patterns.

Recent studies suggest that some patients require phase-controlled layer or volumetric rescanning for sufficiently robust target coverage [Mori et al., 2013, Takahashi et al., 2014]. The advantage of simple slice-by-slice rescanning is that no motion monitoring or assumptions on the breathing frequency are necessary [Bert and Durante, 2011], but the higher required number of rescans might increase treatment times due to reduced beam intensities. Another possibility is to combine rescanning with gating, which was already successfully implemented in clinic [Rossi, 2016].

\subsubsection{RBE and Proton Therapy}

Carbon ions exhibit a radiobiological advantage, especially in the Bragg peak region. However, for high doses as used here the effect of RBE is not well documented and is subject to ongoing research [Friedrich et al., 2014]. For these high doses RBE for carbon ions should approach a value between 1 and 2 [Carabe-Fernandez et al., 2007], which is in agreement with values in our study (around 1.1). 
Coincidently, RBE values in the target at high doses are similar to those used clinically in proton therapy. Carbon-ions show considerably lower lateral scattering though, which should result in even better OAR sparing than protons. Our results are in agreement with several in silico studies comparing SBRT and proton therapy for NSCLC [Roelofs et al., 2012, Kadoya et al., 2010, Register et al., 2010]. Furthermore, a study made by Kadoya reached the same conclusion as our study, that patients with larger CTV and/or multiple CTVs would receive less dose from proton therapy [Kadoya et al., 2010]. A recent phase II trial for patients with multiple sites of extracranial disease showed good results for photons [Iyengar et al., 2014], however, based on the findings of Kadoya et al and our study, proton and/or carbon-ion therapy might result in even better outcome.

\subsubsection{Study limitations}

The 4D dose calculations were based on a regular breathing pattern, which typically varies during patient treatment and/or between 4D-CT acquisition and actual treatment [Verma et al., 2010, Malinowski et al., 2011]. A possible solution was proposed by Boye et al. to get motion information from 4D magnetic resonance imaging (4DMRI) and use it in 4D dose calculations [Boye et al., 2013].

Furthermore, SBRT treatment plans were done on a static case in contrast to a 4D dose calculation done for PT. This should not influence the results of our study, since motion has a smaller impact on photon dose distributions [Zou et al., 2014], whereas it is imperative in PT dose calculations [Bert and Durante, 2011].

There were also differences in treatment planning. PT plans were done by a single person in a research setting, whereas SBRT plans were made by different people under clinical conditions with the requirement to finish the plans on time.

Slight changes also existed between the planning CT, used for SBRT treatment plans and 4D-CT used for PT treatment plans, even though 4D-CT was usually acquired right after the planning CT. The propagation of contours from the planning CT to the 4D-CT and also for the 4D dose calculation rely on deformable image registration (DIR), where even small changes can effect 4D dose distribution [Kashani et al., 2008]. Results from DIR were thoroughly checked and results were presented in Chapter 2. However, the transformation of the dose with DIR is a debated topic and might jeopardize the simulated results, especially with respect to the 4D target coverage. On the other hand, dose differences in OARs were large and should be robust against vector field errors in the order a few $\mathrm{mm}$. Nevertheless, further studies are warranted, possibly using advanced moving phantoms for an experimental validation [Perrin et al., 2014] and finally also clinical trials. First patients are being treated in thoracic and abdominal regions with an active beam scanning at the National Institute for Radiological Sciences (NIRS) in Japan [Mori et al., 2016]. 


\subsubsection{Application}

Scanned carbon ion therapy is available only in a limited number of clinics, mainly due to the considerably higher cost in comparison to photon linacs. Therefore a careful patient selection appears sensible. Patients with larger and multiple lesions where SDRT might be limited due to OAR constraints could be referred to carbon centers. In this study, already lesions larger than $2.5 \mathrm{~cm}^{3}$ were found to benefit significantly stronger from PT.

\subsection{Conclusion}

SBRT and PT both achieved satisfactory target dose. In most patients PT deposited less dose in all OARs (including heart, spinal cord, esophagus, trachea and aorta). Patients with multiple lesions and/or with large target volumes might be preferentially selected for particle therapy. 


\section{Intensity modulated particle therapy for multiple targets}

\section{Contents}

4.1. Introduction $\ldots \ldots \ldots \ldots \ldots \ldots \ldots \ldots \ldots \ldots \ldots$

4.2. Materials and Methods . . . . . . . . . . . . . . 84

4.2.1. Patient data . . . . . . . . . . . . . . . . 84

4.2.2. Multiple targets . . . . . . . . . . . . . . . . 86

4.2.3. Optimization techniques $\ldots \ldots \ldots \ldots \ldots \ldots$. . . . . . 87

4.2.4. Treatment planning . . . . . . . . . . . . . . . . . . 89

4.2.5. Dose escalation . . . . . . . . . . . . . . . . . 90

4.2.6. Data evaluation . . . . . . . . . . . . . . . . . . 90

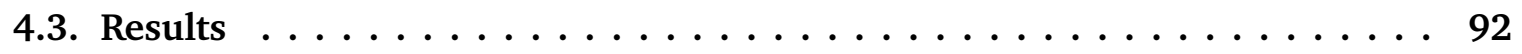

4.3.1. Target Coverage . . . . . . . . . . . . . . . . 92

4.3.2. Dose in OARs . . . . . . . . . . . . . . . . . . . 93

4.3.3. Dose escalation . . . . . . . . . . . . . . . . . 95

4.4. Summary and Discussion . . . . . . . . . . . . . 96

4.5. Conclusion ........................ 98

\subsection{Introduction}

Lung cancer is the leading cause of cancer-related death, with approximately 160000 deaths in the U.S. in 2014 [Siegel et al., 2014]. More than half of all patients with lung cancer are diagnosed with stage IV non-small cell lung cancer (NSCLC) [Ramalingam and Belani, 2008, Iyengar et al., 2014]. The prognosis for stage IV NSCLC is poor, with only 12 months median survival after first line chemotherapy [Socinski et al., 2013].

A stereotactic body radiation treatment (SBRT) shows good results for treating NSCLC [Baumann et al., 2009, Fakiris et al., 2009, Grutters et al., 2010, Greco et al., 2011]. Furthermore, several studies have shown that SBRT can be used in the setting of limited metastatic disease [Rusthoven et al., 2009, Villaruz et al., 2012, Salama et al., 2012, Iyengar et al., 2014]. 
Passive scattering particle therapy has also proved as an effective treatment for NSCLC [Grutters et al., 2010, Tsujii and Kamada, 2012] and it could be considered an alternative to the standard photon treatment.

It was shown in Chapter 3 that scanned carbon ions (PT) could also be used as a treatment modality for NSCLC. One of the conclusions of the study shown in Chapter 3 was that patients with multiple disease sites would especially benefit from PT compared to SBRT. However, limitations of this study were the small number of patients (4) and a single-field uniform optimization (SFUD) used for treatment planning. Several vital organs, beside the lungs, need to be considered in the treatment planning for NSCLC patients, such as heart, spinal cord, esophagus and large vessels. Due to overlapping entry channels SFUD is limited in treating NSCLC, especially in patients with a tumor in close vicinity to an vital organ. It is not possible to create clinically acceptable treatment plans with SFUD for such complex geometry.

We hypothesize that intensity modulated particle therapy (IMPT), permits to calculate adequate treatment plans. Furthermore, IMPT should be able to provide a single fraction scheme in patients, where SBRT is limited by OAR constraints.

The treatment of lung cancer patients with multiple disease sites was investigated using a state of the art 4D IMPT optimization. Treatment plans were generated with two different 4D optimization techniques and compared with SBRT plans, which were actually used for the treating patients.

\subsection{Materials and Methods}

The 4D extension of GSI's treatment planning system TRiP98 [Krämer and Scholz, 2000, Richter et al., 2013] was used and modified to create the needed treatment plans. A description of the modifications and tools used will be given here, alongside with the patient data.

\subsubsection{Patient data}

In this study, 8 patients with 2 - 5 lung metastases, summing up to 24 metastases in total, were included. The median lesion size was $4.2 \mathrm{~cm}^{3}$ (25-75\% $\left.2.4-22.2\right)$ and the median peak-to-peak motion was $5.9 \mathrm{~mm}(2.7$ - 8.1). Further details are given in Table 4.1. The target motion and PT treatment planning were based on a 4D-CT, consisting of 10 phases $(0-9)$, with phase 0 (end-inhale) chosen as a reference phase. A registered positron emission tomography (PET) scan was used to delineate the clinical target volumes (CTV).

Patients 1 - 3 had no OAR in CTV vicinity (closer than $10 \mathrm{~mm}$ ), while patients $4-8$ had at least one. 
All patients were treated with SBRT at the Chamaplimaud Center for the Unknown, Lisbon (Portugal), with different fraction schemes. The number of fractions and doses delivered are given in Table 4.1.

Table 4.1.: Target characteristics, with CTV volumes, peak-to-peak motions, fractionation schemes and number of fields used for PT treatment planning. Last column shows which OARs were present in the target vicinity (closer than $10 \mathrm{~mm}$ ). SA stands for smaller airways and esoph. for esophagus.

\begin{tabular}{|c|c|c|c|c|c|c|}
\hline Patient & Target & Volume $\left(\mathrm{cm}^{3}\right)$ & $\begin{array}{l}\text { Peak-to-peak } \\
\text { motion [mm] }\end{array}$ & $\begin{array}{c}\text { Fractionation } \\
\text { scheme }\end{array}$ & $\begin{array}{l}\text { Number } \\
\text { of fields }\end{array}$ & $\begin{array}{c}\text { OAR in } \\
\text { proximity }\end{array}$ \\
\hline \multirow{2}{*}{1} & $\mathrm{a}$ & 10.2 & 3.4 & 1 x 24 Gy & 2 & \\
\hline & $\mathrm{b}$ & 14.4 & 2.8 & 1 x 24 Gy & 2 & \\
\hline \multirow{5}{*}{2} & $\mathrm{a}$ & 3.8 & 5.8 & 1 x 24 Gy & 2 & \\
\hline & $\mathrm{b}$ & 4.3 & 0.8 & 1 x 24 Gy & 2 & \\
\hline & c & 2.7 & 3.4 & 1 x 24 Gy & 2 & \\
\hline & d & 3.1 & 2.1 & 1 x 24 Gy & 2 & \\
\hline & e & 0.5 & 0.5 & 1 x 24 Gy & 2 & \\
\hline \multirow{2}{*}{3} & $\mathrm{a}$ & 139 & 0.6 & 1 x 24 Gy & 3 & \\
\hline & $\mathrm{b}$ & 9.2 & 2.0 & 1 x 24 Gy & 2 & \\
\hline \multirow{2}{*}{4} & $\mathrm{a}$ & 4 & 9 & 3 x 9 Gy & 5 & SA, esoph., heart \\
\hline & b & 0.8 & 7.8 & 1 x 24 Gy & 2 & \\
\hline \multirow{4}{*}{5} & $\mathrm{a}$ & 3.4 & 5 & 1 x $24 \mathrm{~Gy}$ & 3 & \\
\hline & b & 2.4 & 4.4 & 1 x 24 Gy & 2 & \\
\hline & c & 2.0 & 6.3 & 1 x 24 Gy & 2 & Heart \\
\hline & $\mathrm{d}$ & 2.4 & 6.4 & 1 x 24 Gy & 2 & Heart \\
\hline \multirow{2}{*}{6} & $\mathrm{a}$ & 20.6 & 7.4 & 1 x 24 Gy & 4 & SA \\
\hline & b & 27.1 & 6.0 & 1 x 24 Gy & 5 & SA \\
\hline \multirow{2}{*}{7} & $\mathrm{a}$ & 2.3 & 12 & 1 x 24 Gy & 2 & \\
\hline & $\mathrm{b}$ & 0.4 & 11.8 & 5 x 7 Gy & 5 & $\begin{array}{l}\text { Heart, esoph., } \\
\text { stomach }\end{array}$ \\
\hline \multirow{5}{*}{8} & $\mathrm{a}$ & 136 & 12 & 3 x 9 Gy & 2 & Heart \\
\hline & b & 12.4 & 2.5 & 1 x 20 Gy & 2 & \\
\hline & c & 123 & 14 & 3 x 9 Gy & 2 & Heart \\
\hline & $\mathrm{d}$ & 80.7 & 17 & 1 x 22 Gy & 3 & \\
\hline & $\mathrm{e}$ & 86.7 & 6.6 & 1 x 20 Gy & 3 & SA \\
\hline
\end{tabular}




\subsubsection{Multiple targets}

The TRiP98 optimization works on minimizing the residual of a nonlinear equation system [Krämer and Scholz, 2000]. The cost function $E(N)$ for the particle number $\vec{N}$ is given by :

$$
E(\vec{N})=\sum_{i \in T}\left(D_{\text {plan }}^{i}-D_{a c t}^{i}(\vec{N})\right)+\theta\left(D_{a c t}-D_{\max }\right) w_{O A R} \sum_{j \in O A R}\left(D_{a c t}^{i}(\vec{N})-D_{\text {Max }}\right)
$$

For a CT voxel $i$ and $j$ in the target $T$ and the OAR, respectively; $D_{\text {plan }}, D_{a c t}$ and $D_{\max }$ are the planned, actual and maximum allowed dose, respectively; $\theta$ is the Heaviside step function and $w_{O A R}$ is an OAR specific weight.

The $D_{a c t}(\vec{N})$ is calculated as

$$
D_{a c t}(\vec{N})=\sum_{k=1}^{n} R B E(N) c_{i k} N
$$

The coefficient $c_{i k}$ gives the dose deposition at a voxel $i$ of a pencil beam $k$, with $n$ being the number of pencil beams and RBE is the relative biological effectiveness, calculated with the local effect model or LEM [Elsaesser et al., 2010] .

There is no restriction for the number of targets or fields in the minimizing function, so the first part of Eq. 4.1 can be expanded to:

$$
E(\vec{N})=\sum_{T} \sum_{i \in T}\left(D_{\text {plan }}^{i, T}-\sum_{k=1}^{n} c_{i k} N\right)
$$

However, the setup of raster points in TRiP98 allowed only one target. It was therefore expanded in a way that a field was designated to a specific target, as displayed in Fig 4.1. Raster points for each field are created only around the designated target. All fields contribute dose to all voxels in the optimization. Specifically, $k$ in Eq. 4.3 runs over all pencil beams. Because the optimization function was not changed, all TRiP98 4D functionalities could be used, as explained in the next sections. 
(a)

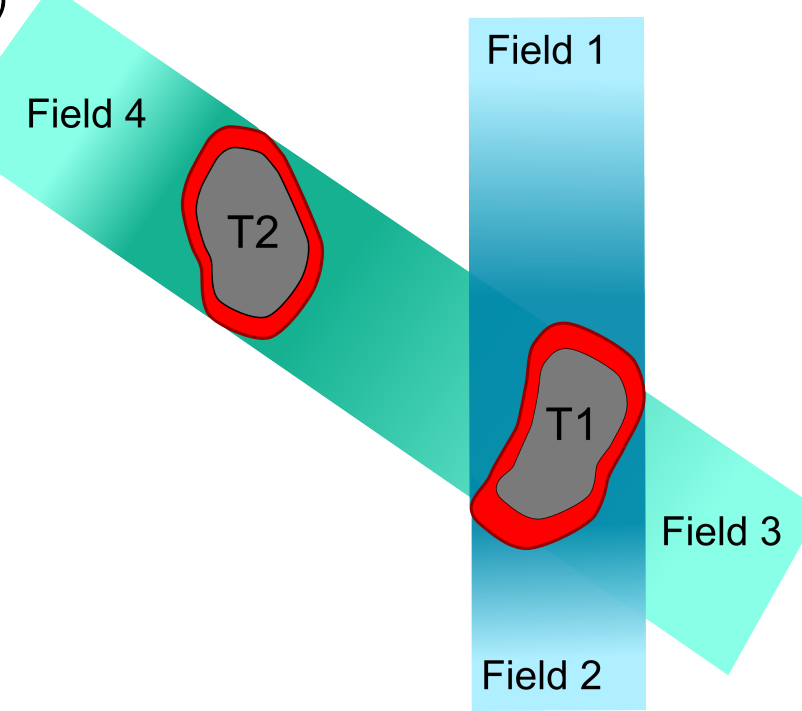

(b)

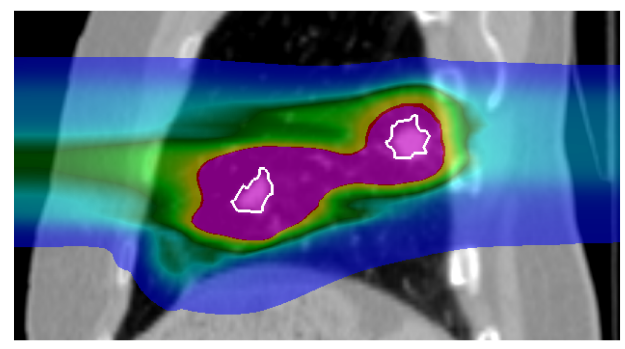

Dose (\%)

100

75

(c)

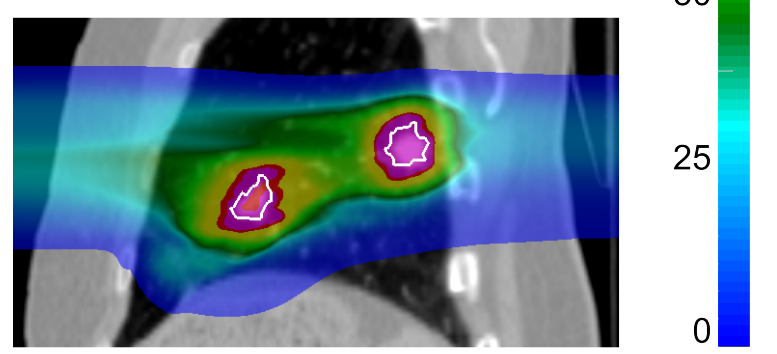

Figure 4.1.: Optimization of multiple targets. (a) Fields 1 and 2 are designated to target $\mathrm{T} 1$ and fields 3 and 4 to target T2. Optimization takes into account all target voxels and contributions from all fields. An example is shown in (b) and (c), where targets were optimized individually (b) and together (c). The dose in (b) reaches almost $200 \%$ in the healthy tissue.

\subsubsection{Optimization techniques}

Investigation of two different optimization techniques to handle range changes in moving tumors was made. For each patient, two sets of plans were created: a field-independent ITV (ITV) and a 4D optimization (4Dopt).

- Field-independent ITV: The water-equivalent path length ITV (WEPL-ITV) is different for each field, creating unnecessary margins when combining WEPL-ITV from different fields (see Fig. 4.2a). Graeff et. al [Graeff et al., 2012] proposed a solution to include range margins into the field description itself, instead of creating a bigger PTV.

Thus, all fields have the same target in the optimization, permitting simultaneous optimization. Treatment plans were made for all targets with IMPT on a ITV in the reference phase. Additionally the target in motion state 5 (end-exhale) was included in the optimization to make the plan more robust against range changes in different motion states.

- 4D Optimization: To include WEPL change specific to each motion state, a 4D optimization was used. 4Dopt uses a WEPL-ITV for raster setup, however the actual optimization is performed on each target voxel in each motion state $m$. The optimization function thus changes to [Graeff et al., 2012]: 


$$
E(\vec{N})=\sum_{m=1}^{M} \sum_{T_{m}} \sum_{i \in T_{m}}\left(D_{\text {plan }}^{i}-\sum_{k=1}^{n} c_{i k m} N\right)
$$

All targets were treated with IMPT and 4D optimization. Due to the large optimization problem for targets $3 \mathrm{a}-\mathrm{b}, 5 \mathrm{a}-\mathrm{d}, 6 \mathrm{~b}, 8 \mathrm{a}$ and $8 \mathrm{c}$ (targets had a big volume or OARs were included besides targets in the optimization), a subset of motion states was used in optimization [Graeff et al., 2012]. To cover most of the different tumor positions, two extreme motion states ( 0 and 5 ) and an intermediate position (7) were chosen.

The same number of fields and the same field angles were used in both techniques.

To reduce the optimization problem, only selected large OARs, such as the heart or the esophagus, were used. Large OARs were manually cropped to the region close to the target. The dose, however, was calculated on the whole OAR to ensure the validity of results.

For targets with different fractionation scheme (targets 8a-e), TRiP98 was modified to include an option, allowing specific dose fractions for specific targets.
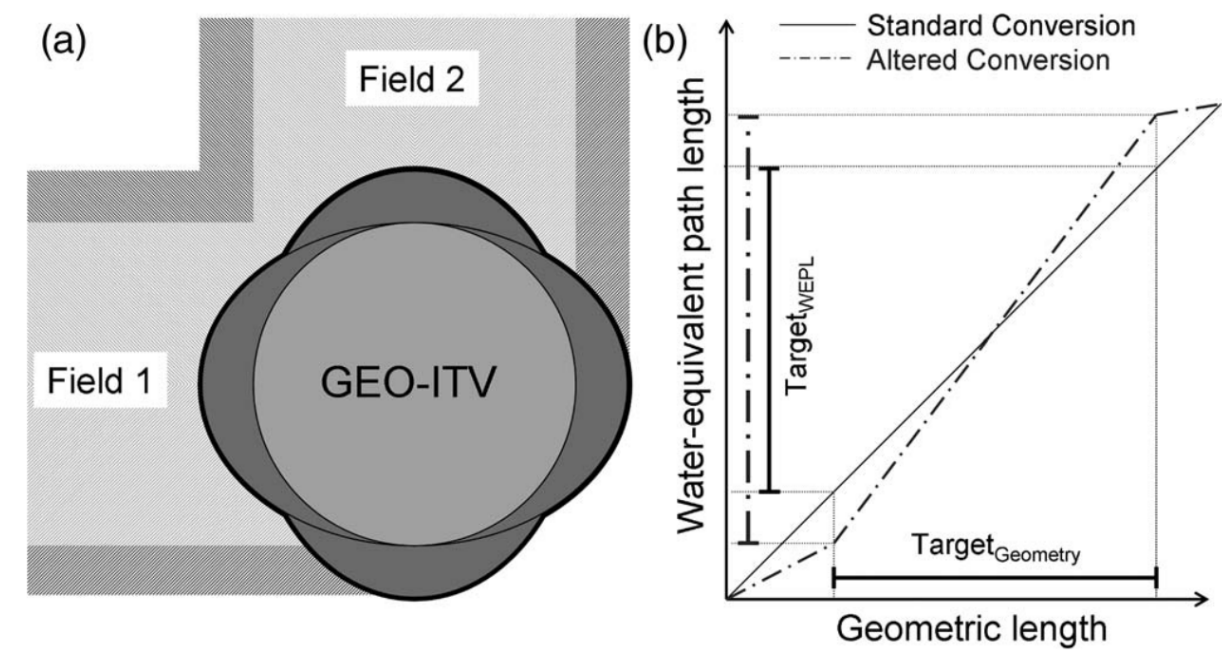

Figure 4.2.: A schematic presentation of the ITV. (a) The dark gray ellipses show the margins needed for specific fields to account for range changes in different motion states. When a common target volume for two perpendicular fields is generated ( $v$ black contour) it creates unnecessary lateral extension of both fields, as shown by the dark gray entry channels. A solution is shown in (b). Rather than using standard, geometric margins, both fields use the same geometry, however the conversion of geometry to WEPL is altered for each field. The plot in (b) shows the standard (solid line) and an altered conversion (dashed-doted line) for a beam passing a homogeneous CTV. The altered conversion increases the WEPL extent and thus implicitly increasing margins for a single field only. Figure taken from [Graeff et al., 2012] 


\subsubsection{Treatment planning}

An isotropic margin of $3 \mathrm{~mm}$ was added to each CTV to account for uncertainties in treatment delivery. A WEPL-ITV was constructed on the CTV with margins for each individual field, which was than used either in the optimization (ITV) or for the raster setup (4Dopt). Due to large memory demands, the targets in each lung were optimized separately.

The planning objective was $99 \%$ of each target volume should receive at least $100 \%$ of the planned dose $\left(\mathrm{D}_{99} \% \geq 100 \%\right)$. Two dose limitation were used for OARs, as defined in the AAPM task group [Benedict et al., 2010]. The first limitation was the maximum dose to a single voxel $D_{\text {Max }}$ and the second the maximum dose deposited to a specific OAR volume $D_{\text {Threshold }}$. All limits are summarized in Table B.1.

After the optimization the 4D-dose was calculated for two motion periods (3.6 sec and 5.0 sec) and two starting phases $\left(0^{\circ}\right.$ and $\left.90^{\circ}\right)$ as explained in Section 3.2.4. The relative biological effectiveness (RBE) was calculated with LEM IV [Elsaesser et al., 2010]. Alpha beta ratio of 6 and 2 was used for the target and normal tissue, respectively.

The motion was mitigated by applying slice-by-slice rescanning to each plan. The number of rescans was limited by the number of particles in a single raster point, which should not be lower than 8000 due to the monitoring precision. The maximum number of rescans was limited to 20 .

A detailed explanation of the SBRT treatment planning is given in Section 3.2.3.

For patients 4 - 7 OAR doses could not be sufficiently reduced in optimization. It was necessary to add margins to the OAR and then subtract the OAR plus margins from the target. For SBRT the OAR plus margins was subtracted from PTV, which included $3 \mathrm{~mm}$ isotropic margins on a geometrical ITV. In PT geometrical ITV was not used, so in each of the 10 motion states, OAR plus margins was subtracted from CTV plus $3 \mathrm{~mm}$.

In the first try the OAR was included in the optimization with different weights $\left(w_{O A R}\right.$ in Eq 4.1) and without any subtraction from the target. For any 3D treatment plan with an acceptable dose distribution after the optimization, a 4D dose was calculated and OAR and target dose were inspected. If the plan was rejected, the optimization was repeated, adding the OAR subtraction from the target volume. 


\subsubsection{Dose escalation}

A single fraction of 24 Gy could not be used in SBRT treatment for targets 4a, 7b and 8a-e due to OAR dose constraints. For these targets additional PT plans were generated with 1 x 24 Gy fractionation scheme, in order to estimate if PT could respect OAR constraints for these targets, while delivering 1 x 24 Gy.

\subsubsection{Data evaluation}

For a comparison of the target coverage, the minimum dose in $99 \%$ of the target volume $\left(D_{99 \%}\right)$

was evaluated. $D_{\text {Max }}$ and $D_{\text {Threshold }}$ were used in the OAR dose comparison. $D_{\text {Max }}$ and $D_{\text {Threshold }}$ were normalized to the respective limits in the fractionation scheme used, see Table B.1. Additionally, the volume receiving $20 \%$ of the planned dose $\left(V_{20 \%}\right)$ was used to assess the ipsilateral lung dose.

Paired t-tests were performed to compare the dose metrics mentioned between SBRT, ITV and 4Dopt. A p-value $<0.05$ was considered as significant. 


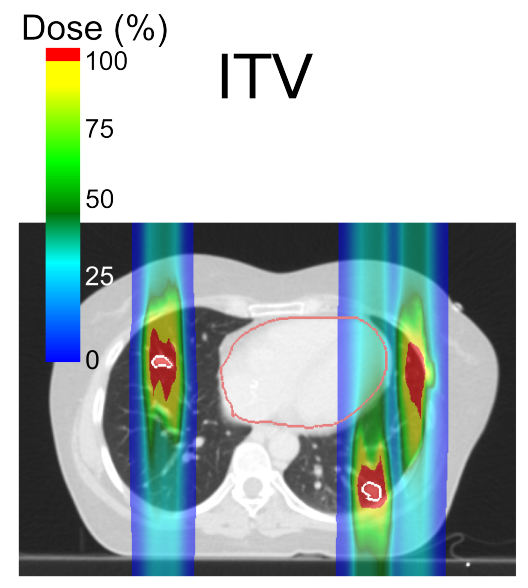

4Dopt

\section{SBRT}

\section{Pat 2}
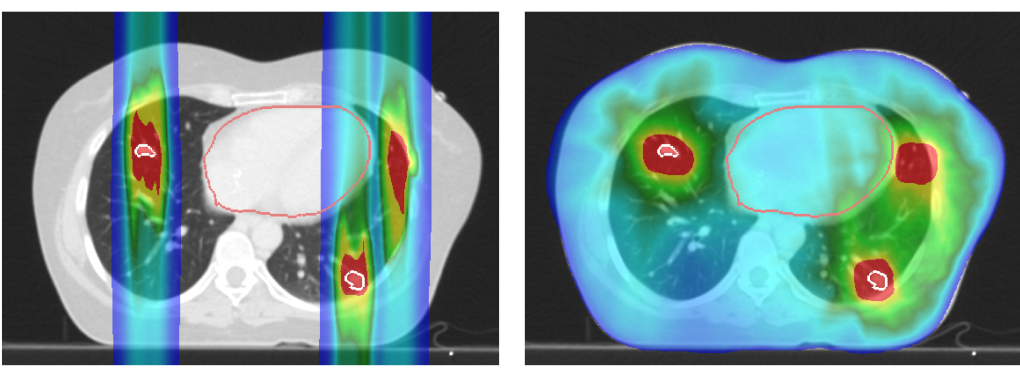

\section{Pat 7}
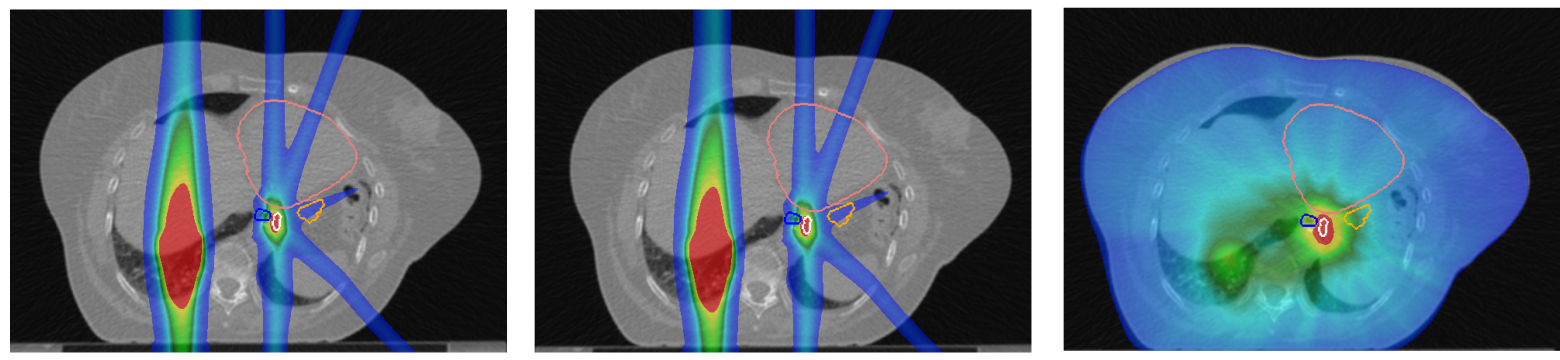

\section{Pat 8}
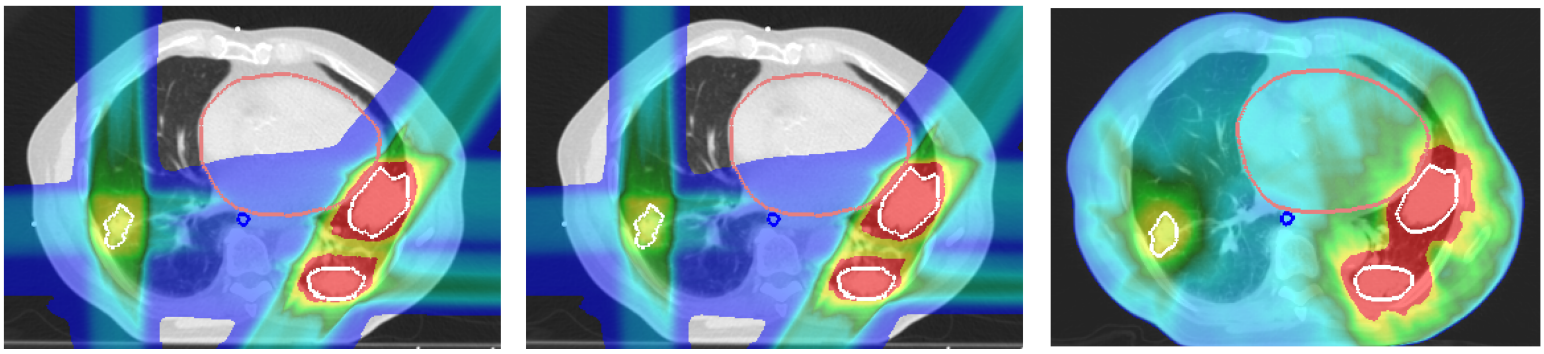

Figure 4.3.: Treatment plans for ITV (left), 4Dopt (middle) and SBRT (right) for patients 2 (top), 7 (middle) and 8 (bottom). CTV, heart, esophagus and stomach contours are outlined in white, red, blue and orange, respectively. The patient 7 image is magnified to the target $7 \mathrm{~b}$ location. Patient 2 has 5 disease sites with no OARs in target vicinity. Patient 7 had poor target coverage with PT due to a large target motion and OAR proximity. A $1 \times 24$ Gy plan could be generated for patient 8 with PT, while SBRT was limited by the dose deposited in the heart. Therefore the fractionation scheme was adapted and 2 targets were treated with $3 \times 9$ Gy, 2 with 1 x 20 Gy and one with 1 x 22 Gy. 


\subsection{Results}

An example of different treatment plans for three patients are shown in Fig. 4.3.

\subsubsection{Target Coverage}

The results for CTV $D_{99 \%}$ for all patients are shown in Table 4.2. All SBRT plans were approved by a physician, even though the prescribed dose for patients 4 - 6 was not met due to an OAR proximity. Target $7 \mathrm{~b} D_{99 \%}$ for PT was below prescription and SBRT delievered full dose. Average CTV $D_{99 \%}$ was 97, 95 and 98\% for ITV, 4Dopt and SBRT, respectivelly, There was a significant difference between ITV and 4Dopt and SBRT and 4Dopt.

Table 4.2.: CTV $D_{99 \%}$ for tITV, 4Dopt and SBRT for 8 patients. The results for ITV and 4Dopt are shown as median (range) across the different motion types.

\begin{tabular}{|c|c|c|c|c|}
\hline \multirow{2}{*}{ Patient } & \multirow{2}{*}{ Target } & \multicolumn{3}{|c|}{ CTV $D_{99 \%}(\%)$} \\
\hline & & ITV & 4Dopt & SBRT \\
\hline \multirow{2}{*}{1} & a & $101.0(101.0-101.0)$ & $101.0(101.0-101.0)$ & 100.0 \\
\hline & $\mathrm{b}$ & $101.0(101.0-102.1)$ & $101.0(101.0-101.0)$ & 100.0 \\
\hline \multirow{5}{*}{2} & $\mathrm{a}$ & $101.0(101.0-102.1)$ & $100.0(99.0-102.1)$ & 106.3 \\
\hline & $\mathrm{b}$ & $102.1(102.1-102.1)$ & $102.1(102.1-102.1)$ & 103.1 \\
\hline & c & $101.0(100.0-101.0)$ & $101.6(101.0-102.1)$ & 104.2 \\
\hline & $\mathrm{d}$ & $102.1(101.0-102.1)$ & $102.1(102.1-102.1)$ & 107.3 \\
\hline & e & $101.0(101.0-101.0)$ & $101.0(101.0-102.1)$ & 108.3 \\
\hline \multirow{2}{*}{3} & a & $101.0(101.0-101.0)$ & $101.0(101.0-101.0)$ & 101.0 \\
\hline & $\mathrm{b}$ & $98.4(97.9-99.0)$ & 97.9(97.9 - 97.9) & 102.1 \\
\hline \multirow{2}{*}{4} & $\mathrm{a}$ & $65.3(63.9-69.4)$ & $70.4(68.5-72.2)$ & 66.7 \\
\hline & $\mathrm{b}$ & $101.0(100.0-102.1)$ & $100.5(100.0-102.1)$ & 103.1 \\
\hline \multirow{4}{*}{5} & a & $100.0(99.0-101.0)$ & $100.0(100.0-100.0)$ & 101.0 \\
\hline & $\mathrm{b}$ & $101.6(100.0-102.1)$ & $97.9(96.9-99.0)$ & 101.0 \\
\hline & c & 95.3(94.8 - 96.9) & $94.3(92.7-94.8)$ & 99.0 \\
\hline & $\mathrm{d}$ & $99.0(97.9-99.0)$ & $99.5(99.0-100.0)$ & 94.8 \\
\hline \multirow{2}{*}{6} & a & $89.1(88.5-90.6)$ & $85.4(85.4-87.5)$ & 69.8 \\
\hline & $\mathrm{b}$ & $78.6(77.1-79.2)$ & $72.4(71.9-72.9)$ & 69.8 \\
\hline \multirow{2}{*}{7} & $\mathrm{a}$ & $102.1(102.1-102.1)$ & $99.0(99.0-99.0)$ & 101.0 \\
\hline & $\mathrm{b}$ & 83.9(82.1 - 85.7) & $75.0(75.0-75.0)$ & 100.0 \\
\hline \multirow{5}{*}{8} & $\mathrm{a}$ & $100.0(100.0-100.9)$ & $99.5(99.1-100.9)$ & 105.6 \\
\hline & $\mathrm{b}$ & $101.3(100.0-102.5)$ & $100.0(100.0-101.3)$ & 105.0 \\
\hline & c & $100.0(99.1-100.0)$ & $99.5(97.2-100.0)$ & 106.5 \\
\hline & $\mathrm{d}$ & $102.3(102.3-102.3)$ & $89.8(89.8-90.9)$ & 102.3 \\
\hline & $\mathrm{e}$ & $102.5(102.5-102.5)$ & $91.9(91.3-92.5)$ & 101.3 \\
\hline
\end{tabular}




\subsubsection{Dose in OARs}

$D_{\text {Max }}$ and $D_{\text {Threshold }}$ for 8 OARs are shown in Table 4.3. Dose volume histograms (DVH) for patients 4, 6 and 7 are shown in Fig. 4.4. There was a significant difference between PT and SBRT in $D_{\text {Max }}$ and $D_{\text {Threshold }}$ for heart, spinal cord, esophagus and aorta. No significant difference was observed for $D_{\text {Max }}$ and $D_{\text {Threshold }}$ in the smaller airways. No significant difference was observed in the dose to any OAR between the different motion types or between ITV and 4Dopt. The overall OAR difference for patients between SBRT and ITV was significant, 17 (4 - 52)\% and 27 (8 - 55)\% of OAR limits for $D_{\text {Max }} D_{\text {Threshold }}$, respectivelly. The ipsilateral lung $V_{20 \%}$ was 14.5(0.0 - 48.7), 14.4(0.0 - 43.7) and 29.8 (5.8 - 89.2)\% for ITV, 4Dopt and SBRT, respectively. Both, ITV and 4DITV ipsilateral lung $V_{20 \%}$ was significantely different from SBRT.

The margins used for the OAR subtraction for PT and SBRT can be found in Table C.11.

All treatment plans exceeded the $D_{\text {Max }}$ limit for the smaller airways in patients 4,6 and 8 and for the heart in patient 6 . Additionally, the SBRT esophagus and heart $D_{\text {Max }}$ limits were exceeded in patients 4 and 8 , respectively.

Table 4.3.: OAR $D_{\text {Max }}, D_{\text {Threshold }}$ and ipsilateral lung $V_{20 \%}$ of all patients for ITV, 4Dopt and SBRT. There was a significant difference between PT and SBRT for all OARs, except smaller airways' $D_{\text {Max }}$. $D_{\text {Max }}$ and $D_{\text {Threshold }}$ doses are normalized to the corresponding OAR limits in the fractionation scheme used (see [Benedict et al., 2010]). The data is displayed as median (range).

\begin{tabular}{|c|c|c|c|}
\hline \hline \multirow{2}{*}{ OAR } & ITV & 4Dopt & SBRT \\
\cline { 2 - 4 } & \multicolumn{3}{|c|}{$D_{\operatorname{Max}}(\%)$} \\
\hline heart & $62.0(0.0-100.0)$ & $59.5(0.0-97.0)$ & $82.5(20.0-103.0)$ \\
spinalcord & $13.0(0.0-48.0)$ & $12.0(0.0-55.0)$ & $60.0(21.0-79.0)$ \\
smaller airways & $72.5(0.0-130.0)$ & $71.0(0.0-117.0)$ & $72.5(0.0-171.0)$ \\
esophagus & $9.0(0.0-79.0)$ & $8.0(0.0-99.0)$ & $70.5(20.0-101.0)$ \\
aorta & $17.5(8.0-61.0)$ & $15.0(7.0-61.0)$ & $45.0(15.0-74.0)$ \\
\hline \hline & \multicolumn{3}{|c|}{$D_{\text {Threshold }(\%)}$} \\
heart & $15.5(0.0-59.0)$ & $16.0(0.0-53.0)$ & $62.5(19.0-98.0)$ \\
spinalcord & $11.0(0.0-45.0)$ & $10.0(0.0-53.0)$ & $66.5(28.0-95.0)$ \\
smaller airways & $28.0(0.0-97.0)$ & $26.5(0.0-89.0)$ & $68.5(0.0-99.0)$ \\
esophagus & $1.0(0.0-17.0)$ & $1.0(0.0-20.0)$ & $49.0(17.0-99.0)$ \\
aorta & $5.5(0.0-30.0)$ & $5.5(0.0-28.0)$ & $34.5(12.0-59.0)$ \\
\hline \hline & \multicolumn{3}{|c|}{$V_{20 \%}(\%)$} \\
ipsilateral lung & $14.5(0.0-48.7)$ & $14.4(0.0-43.7)$ & $29.8(5.3-89.2)$ \\
\hline \hline
\end{tabular}




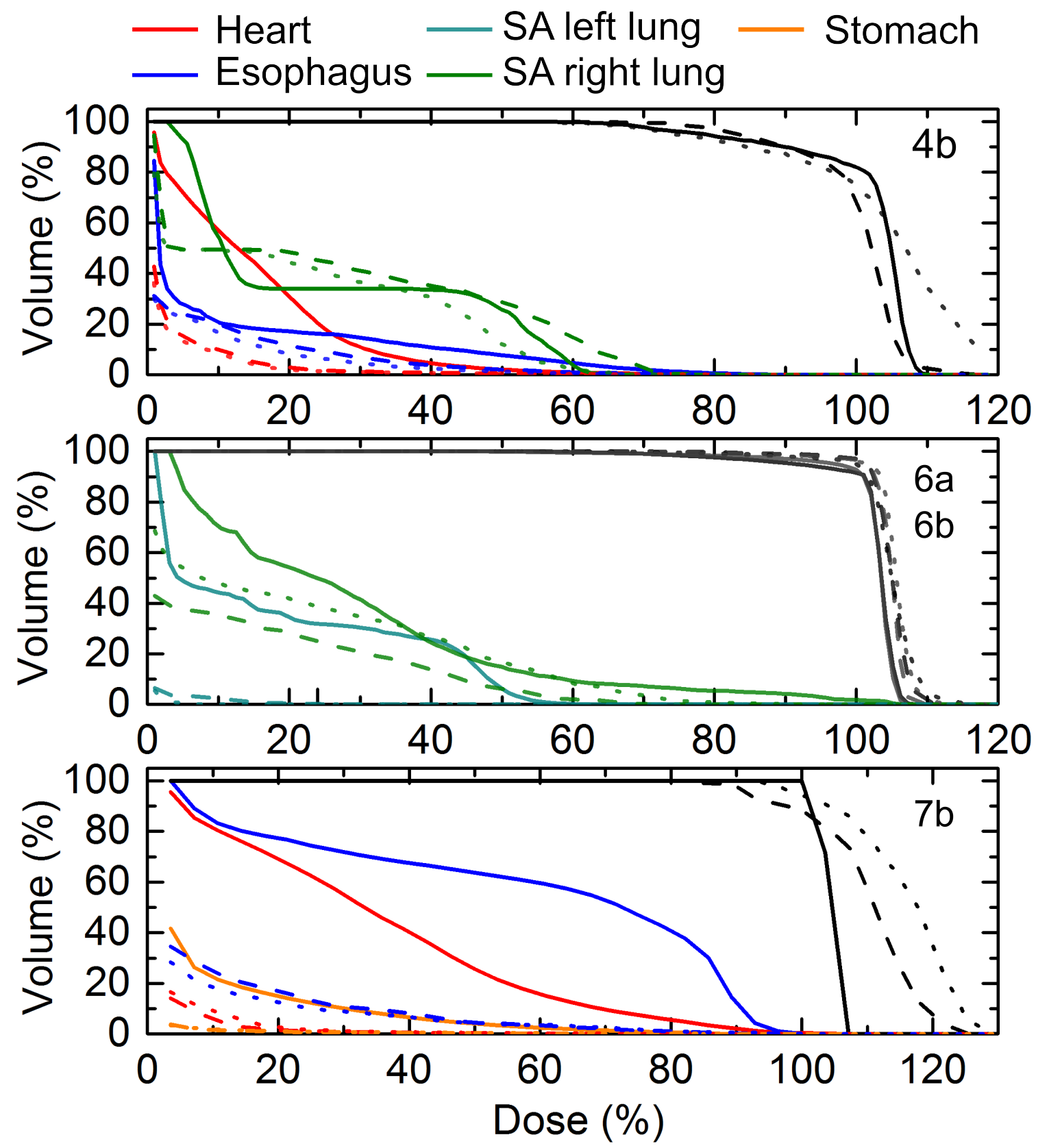

Figure 4.4.: Dose volume histograms for targets $4 b, 6 a, 6 b$ and $7 b$ with relevant OARs. SBRT, ITV and 4DITV are represented by solid, dashed and dotted lines, respectively. The targets are displayed in grayscale, while the OAR colors are shown in legend. SA stands for smaller airways. 


\subsubsection{Dose escalation}

With in PT the 1 x 24 Gy fractionation scheme could be used for targets 8a-e, violating only $D_{\text {Max }}$ for the smaller airways (180\%) and the heart (110\%), to a similar extent as was found acceptable in SBRT. The SBRT for patient 8 was limited by the heart $D_{\text {Max }}$ and $D_{\text {Threshold }}$ which were $102 \%$ and $93 \%$, respectively. SBRT delivered a mean heart dose of 3 Gy in a single fraction (out of three), whereas PT's mean heart dose was $0.8 \mathrm{~Gy}$. The difference in the heart dose can be seen in Fig 4.5 .

For targets $4 \mathrm{a}$ and $7 \mathrm{~b}$ the 1 x 24 Gy fractionation scheme could not be generated with PT. Either the target coverage was low (CTV $D_{99 \%}<50 \%$ ) or the esophagus $D_{\text {Max }}$ and additionally the stomach $D_{\text {Max }}$ for target $7 \mathrm{~b}$ were exceeded.

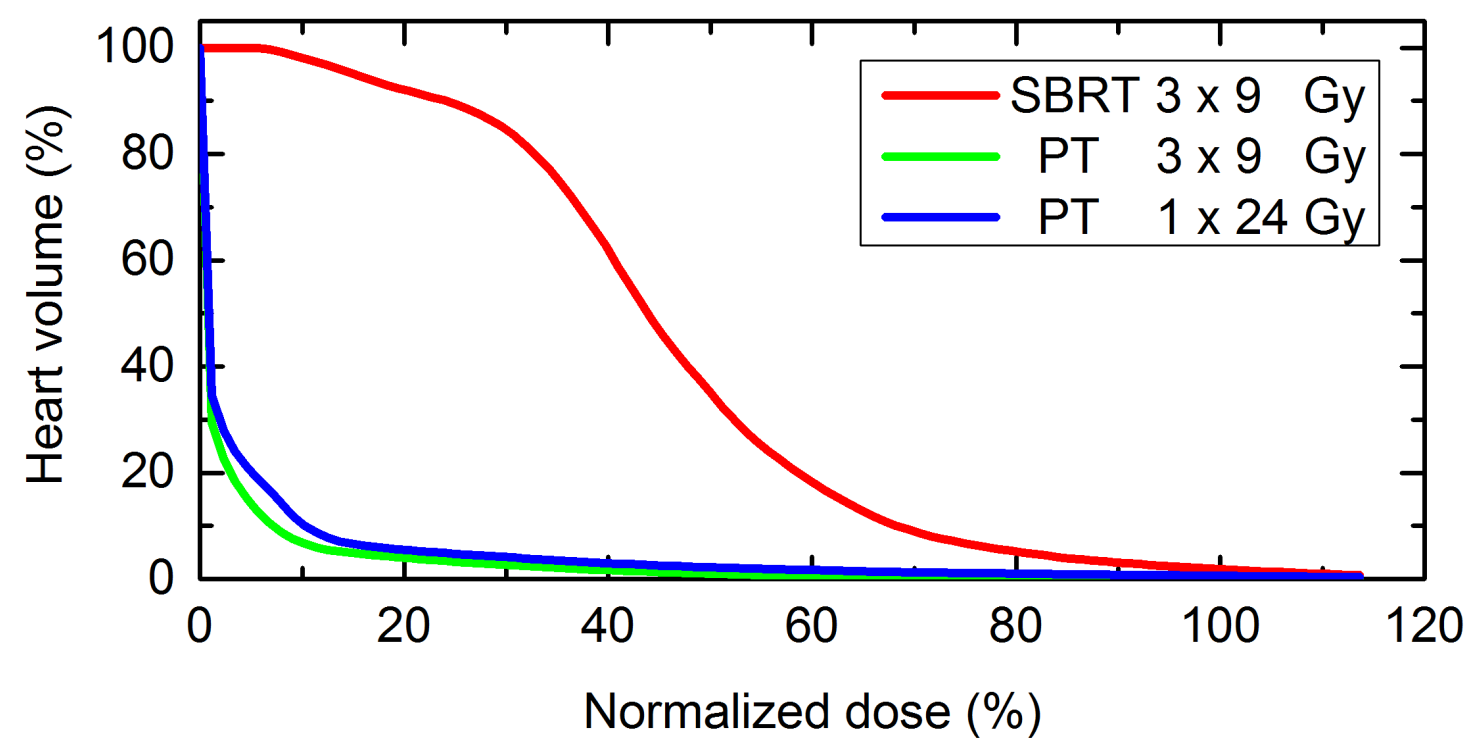

Figure 4.5.: Dose volume histogram for the heart dose of the Patient 8. SBRT (red) plan was delievered in a 3 × 9 Gy fractionation scheme. The ITV PT plans were generated for the same fractionation scheme (green) and with a dose escalation $1 \times 24$ scheme (blue). The dose was normalized to $D_{\operatorname{Max}}$ heart limit in respective fractionation scheme - 30 Gy in 3 x 9 Gy and 22 Gy in 1 x 24 Gy. 


\subsection{Summary and Discussion}

Clinically valid SBRT plans have been compared to PT treatment plans for NSCLC patients with multiple metastases. To the best of our knowledge, this is the first study treating multiple NSCLC metastases with IMPT. A novel approach was used to handle multiple targets, combined with state of the art 4D IMPT treatment planning. Furthermore, 4D PT doses were calculated for different motion types.

PT on average delivered less dose to OARs, while still having comparable target coverage to SBRT. The most important difference was found in the heart dose, with $D_{\text {Threshold }}$ being on average 6 times lower in PT compared to SBRT. A recent trial, RTOG 0617, has shown, that a higher mortality rates could be attributed to higher heart dose for NSCLC patients [Bradley et al., 2015]. Furthermore, as seen in Table 4.3, the median $D_{\text {Threshold }}$ for all OARs is below $30 \%$ and $D_{\text {Threshold }}$ exceeds $90 \%$ in only one OAR in one patient. For SBRT $D_{\text {Threshold }}$ comes close to the limit in all OARs, except the aorta. There was no need to include $D_{\text {Threshold }}$ in treatment planning, whereas it is imperative in SBRT.

For patients with a complex geometry (4 - 8) PT maintained or even improved target coverage in most cases, while reducing doses to OARs. The exception was target $7 \mathrm{~b}$, where CTV $D_{99 \%}$ was low ( $84 \%$ and $75 \%$ for ITV and 4 Dopt, respectively), due to the $D_{\text {Max }}$ constraints of the esophagus and the stomach. The large motion of target $7 \mathrm{~b}(11.8 \mathrm{~mm})$ and the small target volume $\left(0.4 \mathrm{~cm}^{3}\right)$ contributed to a poor PT plan, whereas SBRT was able to deliver the full dose to the target and adhering to OAR constraints. This supports our claim in Chapter 3 that targets with larger volume would benefit most from PT. Furthermore, for small targets with large motion in OAR vicinity, PT generates worse plans than SBRT. It should be noted, however, that integral doses for all OARs are still lower for PT as seen in Fig 4.4. The only limitation for PT is usually the OAR's $D_{\text {Max }}$.

The biggest advantage of PT could be seen in Patient 8 , where the fractionation scheme could be changed to $1 \times 24$ Gy. The large total target volume of Patient 8 could be irradiated with less overall dose and hence significantly reduced the dose to all OARs. Most notably, the heart dose, which was the limitation factor for SBRT. The difference of 2 Gy mean heart dose in a single fraction is tremendous and could influence the potential outcome for the specific patient. Again, this confirms our claim of PT benefit for large targets. Due to the OAR constraints of targets $4 a$ and $7 b$, located adjacent to important serial organs, no fractionation escalation was possible with PT.

There was a small difference in the average target coverage between ITV and 4Dopt. The most notable difference was in targets $8 \mathrm{~d}$ and $8 \mathrm{e}$, where CTV $D_{99 \%}$ was $10 \%$ lower for $4 \mathrm{Dopt}$. For this patient 4Dopt was performed on a subset of the 4D-CT states, which may be inadequate due to the large motion of target $8 \mathrm{e}(17 \mathrm{~mm})$. In a future study, the number of voxels included in optimization should be reduced, without reducing the target coverage. A possible solution would 
be an adaptive dose grid [Prall et al., 2016b]. There was no significant difference between ITV and 4Dopt in the doses to OARs.

Even though PT deposits less dose to OARs with the same or even better target coverage, there is still room for improvement in PT 4D treatment planning. An implementation of multi-criteria objective planning should bring even better dose distribution and bring possibility to choose between trade-offs [Breedveld et al., 2007, Chen et al., 2010]. Additionally, the multiple target optimization in PT would benefit from a shell around PT where the dose would be minimized. Therefore the excessive dose in healthy tissue would be further reduced. An introduction of a shell, however, would further enlarge the optimization problem, which is big already for complex geometries (patient 4 - 8). An adaptive optimization grid could be a possible solution here as well [Prall et al., 2016b].

In Chapter 3 additional range margins to account for range uncertainties could be used in the treatment planning, due to SFUD. Because we did not use field specific PTVs, it was not possible to include range uncertainties in this study. Instead of creating field specific PTVs to include range uncertainties, a solution was proposed to include uncertainties in the optimization process itself [Pflugfelder et al., 2008, Unkelbach et al., 2009, Fredriksson et al., 2011, Chen et al., 2012]. Chen et al. have implemented a robust optimization in a multi-criteria optimization as well [Chen et al., 2012]. Furthermore, in a recent treatment planning study by Liu et al. [Liu et al., 2016b] a 4D robust optimization was demonstrated, with better results over 3D robust optimization for NSCLC patients. However, only the breathing starting phase was used as an uncertainty, whereas different motion types should be considered. The disadvantage of $3 \mathrm{D}$ and $4 \mathrm{D}$ robust optimization is the enlargement of the optimization problem.

Patient $4 D_{\text {Max }}$ esophagus dose ranged over 1.3 Gy across different motion types in 4Dopt, showing the necessity of making treatment plans robust against motion uncertainties, especially in the hypo-fractionated regiment. Furthermore, OAR doses that are under the limits after optimization, may exceed them after calculating the 4D dose. The ITV and 4Dopt approaches take into account range changes in different motion states, however they do not address interplay. This could be solved with a complete 4D optimization [Graeff et al., 2013], where a 4D raster treatment plan is generated and each motion state has a designated treatment plan.

Apart from 4D robust optimization, the effect of the motion could be minimized by using other motion mitigation techniques, such as gating. Furthermore, gating could improve the target coverage, where the planned dose was not met. Gating, together with rescanning, has already been successfully implemented clinically for active beam scanning [Rossi, 2016, Mori et al., 2016] and it might be essential to use it in hypo-fractionated treatment of moving tumors [Richter et al., 2014].

A recent review showed good local control rates between 66 - 92\% for patients treated with SBRT for in-field recurrent tumors [Amini et al., 2014]. However, there were grade 4 and 5 complications present. A study by Trovo et al.showed grade 5 pneumonia in $6 \%$ of patients 
treated [Trovo et al., 2014]. As shown in our study, PT delivers less dose to the OARs, ipsilateral lung in particular, and could hence reduce the number of treatment-related complications.

\subsection{Conclusion}

PT delivers less dose to OARs compared to SBRT in NSCLC patients with multiple disease sites, while maintaining target coverage. One patient with a large total target volume could be irradiated with 1 x 24 Gy, whereas it could not with SBRT. There was a small difference between the two 4D treatment planning techniques.

Patients with multiple NSCLC disease site have a poor prognosis, with a median survival shorter than a year. A treatment with SBRT can prolong the patient's life, however there is a $10 \%$ chance of death due to the severity of the treatment. PT could maintain the SBRT survival rate, while tremendously reducing treatment related side effects. 


\section{Discussion}

This is the first in silico study directly comparing clinical stereotactic body radiation therapy (SBRT) with scanned carbon-ions (PT) for non-small cell lung cancer (NSCLC). Our results show that PT could be considered an alternative to SBRT, with the same tumor coverage and less dose to OARs. Furthermore, the study was expanded to patients with multiple NSCLC disease sites. With a state of the art 4D optimization, intensity modulated particle therapy (IMPT) was able to generate treatment plans with less OAR doses and comparable target coverage to SBRT. It was possible to plan for a a single fraction ablative dose with IMPT for a specific patient with 5 lesions, where SBRT was limited due to excessive heart dose. The mean heart dose could be reduced by an order of magnitude.

The treatment of NSCLC with PT is influenced by interplay effects between the tumor motion and the beam scanning. It was shown that rescanning offers adequate motion mitigation.

PT offers precise dose shaping but it can thus also be more prone to uncertainties. Calculation of time-resolved (4D) doses can be significantly affected by errors in deformable image registration (DIR) [Heath and Seuntjens, 2006]. Special tools were developed in the scope of this thesis to ensure DIR quality assurance (DIRQA). All tools were tested on a large dataset to ensure their validity.

\subsection{Deformable image registration and validation}

A single DIR algorithm was used in this study, B-Spline. In contrast to Demons algorithm, B-Spline should handle large deformations well as present in lung and cardiac 4D-CT [Tang et al., 2013]. The DIR for lung 4D-CT had only small inconsistencies and here B-Spline can be considered sufficient. On the other hand, the results suggest that B-Spline is inadequate for DIR of a pig cardiac 4D-CT. The parameters used in B-Spline DIR were similar in both cases of DIR. This could be improved by systematically investigating the effect of parameters on DIR quality.

The advantage of using open-source software for DIR, as explained in Section 2.2.2 is that different DIR algorithms, optimization metrics and image types can be used. They can be accessed either using existing libraries, such as ITK [Yoo et al., 2002], or by writing designated software [Fedorov et al., 2015]. In the future, different DIR algorithms have to be implemented and tested for various anatomical sites. 
In this study, DIR was used for contour propagation and 4D dose calculations. The 4D dose calculation requires accurate DIR in each voxel, since the dose is propagated with the resulting vector field. This was ensured by calculating the vector field Jacobian and ICE voxel-wise.

Tests in the DIRQA module were divided into two groups - qualitative and quantitative. Qualitative tests are false color and checkerboard; they provide an overview of the DIR result. However they do not give any information of the vector field quality and it is impossible to review the sheer amount of data. An example of the disadvantage of the qualitative test could be seen in the pig cardiac 4D-CTs, where qualitative test did not show any errors in DIR, but errors were observed in the vector fields.

The quantitative tests used in the DIRQA module are landmark distance, absolute difference, Jacobian and ICE. Absolute difference, Jacobian and ICE have undergone an extensive testing. The results suggest that absolute difference gives us the least information about DIRQA, apart that it has to be lower after the DIR. We have shown that bigger deformations yield more deviations in Jacobian and ICE, which was also previously reported [Stanley et al., 2013]. Furthermore, we have confirmed that Jacobian should always be positive for a successful DIR [Rey et al., 2002]. Additionally, our results show that ICE should be smaller than the maximum vector field magnitudes. Any deviations from the mentioned trends should be thoroughly examined.

There are additional vector field validation methods beside Jacobian and ICE, such as vector field curl [Schreibmann et al., 2012], unbalanced energy [Zhong et al., 2007], permutation, and analysis of the variance (ANOVA) tests [Klein et al., 2009]. It was demonstrated in a study by Salguero et al. [Salguero et al., 2011] that DIR errors greater than $1 \mathrm{~mm}$ can lead to large dose errors in high-dose gradient regions. Therefore the DIR accuracy has to be quantified at each image voxel in the high-dose gradient regions. In our study, a focus was given on a complete registration to find potential errors. However, in future studies the regions of interest used should be around the target, where high-dose gradients can occur. Furthermore, the effect of the image and vector field downsampling on DIRQA and on 4D dose calculation should be assessed.

Due to the lack of landmarks in all 4D-CTs, landmark distance was not included in the verification. Two contour based validations, dice similarity coefficient [Varadhan et al., 2013] and Hausdorff distance [Huttenlocher et al., 1993] are planned to be implemented in the DIRQA module. In the literature many approaches have been reported to assess DIRQA with landmarks or contours. A study by Hardcastle et al. [Hardcastle et al., 2012] compared demons and Salient-Feature-Based registration with dice coefficients between propagated and physician drawn contours. A multi-institutional study by Brock et al. [Brock, 2010] compared differences in propagated and oncologist drawn landmarks. A method has been developed by Castillo et al. [Castillo et al., 2009] to automatically identify landmark points in lung patients images. How- 
ever, visual based evaluations are of limited use in regions of uniform image intensity and by the number of the objects being tracked [Kashani et al., 2008, Liu et al., 2012].

\subsection{Treating non-small cell lung cancer with particle therapy}

The results in this thesis suggest that PT could be used as a treatment modality for NSCLC. It delivers a comparable target dose to SBRT, while significantly reducing the dose to OARs. The lower mean heart could be crucial in improving patient survival based on a recent trial from RTOG 0617 [Bradley et al., 2015]. The mean dose to the heart would be on average $1 \mathrm{~Gy}$ smaller with PT than with SBRT. For patients with multiple disease sites, it would be on average 4 Gy smaller, reaching up to 9 Gy. Similar results were observed when comparing protons to SBRT [Georg et al., 2008].

The advantageous dose profile of PT permits to use few, selected fields with narrow entry channels, avoiding the dose bath needed in SBRT to achieve high dose gradients in the target. Hence the benefit of PT is most profound for patients with large total target volume, whether a large single target or multiple targets. Studies suggest, that SBRT is limited for large tumors (radius $>5 \mathrm{~cm}$ ) and multiple primary tumors [Timmerman et al., 2006, Georg et al., 2008, Westover et al., 2012], making PT a promising alternative.

Besides large tumors and multiple primary tumors, SBRT is also limited in treating centrally located tumors and tumors close to the chest wall. In a study done at Francis H. Burr Proton Therapy Center patients who could not be treated with SBRT, due to the scenarios mentioned, were treated with passive proton beam in 3 - 5 fractions, delivering 42 - 50 Gy [Westover et al., 2012]. They observed similar tumor local control rates as in SBRT (100\% in a two year follow-up) with limited toxicities. It should be stressed that these patients were rejected from SBRT treatment due to the complexity and regardless proton therapy achieved similar results to SBRT.

In addition to a narrow entry channel, PT has sharper dose gradients and can conform the dose better to the target. Fig 3.3 shows that $80 \%$ of the targets have $D_{99 \%}$ between $100-107 \%$ and 100 and $102 \%$ for SBRT and PT, respectively. Sharper dose gradients also enable less dose to the surrounding tissue. Nevertheless, a fraction escalation was possible only in one patient out of three. The limitation in the two patients with unsuccessful fraction escalation was the esophagus maximum single point dose $D_{\text {Max }}$, which is 15 Gy in 1 x 24 Gy scheme. In both patients the esophagus was closer than $2 \mathrm{~mm}$ to the target, making the limitation impossible to respect without sacrificing target dose. Furthermore, for one of these two patients, PT could not deliver planned target dose, whereas SBRT could. Beside complex geometry, the tumor had a small volume and large motion. This patient exhibits the advantage of SBRT over PT.

PT has to take into account particle range uncertainty, which can come from the conversion of HU to stopping power [Schneider et al., 1996] or from anatomical changes in the patient [Unkelbach et al., 2009]. We included range uncertainties with expansion of the target in 
beam's eye view, which resulted on average in 1.5 times bigger target volume for PT compared to SBRT. Another way to include range and other uncertainties is robust optimization, resulting in IMPT plans more resilient to uncertainties [Unkelbach et al., 2009, Chen et al., 2012]. We are planning to include robust optimization in a future study, where SFUD, IMPT and robust IMPT plans will be compared for NSCLC patients.

While tumor motion influences photon treatment, it can be mitigated with proper margins [Zou et al., 2014]. On the other hand, effects can be substantial when treating moving targets with scanned particle therapy [Bert et al., 2008]. It was shown in this thesis that rescanning is an adequate motion mitigation technique. However, rescanning has a degree of uncertainty, especially regarding OAR $D_{\text {Max }}$. In a hypofractionated treatment these limits are strict and the exact dose to the OAR must be known. A possible solution would be to simulate rescanning and 4D delivery in the optimization process itself. Such a solution is not yet feasible due to the complexity of the problem. Another solution could also be phase-controlled rescanning with greatly reduced uncertainty in the mitigation outcome [Mori et al., 2013, Takahashi et al., 2014]. However, it requires motion monitoring and complicates the treatment delivery.

Gating is a commonly used motion mitigation technique in both photon and particle treatment. While it provides less motion-induced dose errors, it prolongs the treatment time. A recent study by Zhang et al. [Zhang et al., 2015] included different breathing patterns, obtained from MRI, on a 4D-CT and calculated 4D doses for liver cancer patients treated with proton therapy. They have shown that a gating window of $3 \mathrm{~mm}$ can result in a $10 \%$ efficiency of a duty-cycle, substantially prolonging the treatment. Additionally, they have shown that neither volumetric or slice-by-slice rescanning could achieve good target coverage. However, this was obtained with a combination of gating and rescanning. Their results suggest that a combination of gating and rescanning would currently be the best solution for treating NSCLC patients with PT.

Between rescanning, gating and beam tracking is the most precise technique, since it requires no internal target margins [Bert and Durante, 2011]. Current clinical implementations of tracking in photon radiotherapy [Kilby et al., 2010, Keall et al., 2014] can not be directly used in particle therapy, since they only provide the position of single internal points. Fassi et al. [Fassi et al., 2015] were able to account for inter- and intra-fractional variability of patient's anatomical configuration with a designated modeling technique [Fassi et al., 2014]. The measured median of water-equivalent path length in target was within $2 \mathrm{~mm}$ of a simulated one. For an actual clinical implementation it will be necessary to test the model on a large patient dataset.

All three techniques, rescanning, gating and beam tracking, essentially adapt 3D treatment plans to a 4D situation and thus have limitations. Full 4D-optimization, on the other hand, creates a 4D treatment plan, with each motion state in the 4D-CT having a designated treatment 
plan. A full 4D-optimization has been successfully implemented and verified experimentally at GSI [Graeff et al., 2013].

Recent advances in photon radiotherapy allow the use of non-coplanar beams, a so-called $4 \pi$ optimization [Dong et al., 2013b]. A study by Dong et al. [Dong et al., 2013a] showed that $4 \pi$ yielded better target coverage and OAR sparing than SBRT for NSCLC patients. They have reported a reduction of $D_{\text {Max }}$ in the heart, the esophagus and the spinal cord by $32 \%, 72 \%$ and $53 \%$, respectively, showing the potential of a $4 \pi$ optimization. According to this thesis, PT is able to reduce the $D_{\operatorname{Max}}$ even further, with a reduction of $57 \%, 87 \%$ and $83 \%$ for the heart, the esophagus and the spinal cord, respectively. The numbers, however, should be compared with caution, since they were obtained from a different set of patients. A future study, directly comparing SBRT, $4 \pi$ and PT for NSCLC is thus warranted.

In a recent phase II study by Iyengar et al. [Iyengar et al., 2014] patients with stage IV NSCLC were treated with SBRT and chemotherapy. They have irradiated 52 targets in 24 patients, 16 of them had more than one target. The results were promising, with 20 months median overall survival, compared to 9 months when treating with chemotherapy only [Tsao, 2016]. Results in this thesis show that patients with multiple disease sites would especially benefit from PT. Based on the poor prognosis of stage IV NSCLC patients and on the results published by Iyengar et al., stage IV NSCLC patients could be eligible candidates for PT treatment. Additionally, such patients usually exhibit chronic obstructive pulmonary disease and less dose to the lung is warranted [Westover et al., 2012]. This further supports our claim, since our study showed substantial differences in the doses to the ipsilateral lung ( $V_{20 \%}$ was on average $15 \%$ smaller in PT for patients with multiple disease sites) and contralateral lung as well - 70\% of patients did not receive any dose to the contralateral lung, whereas SBRT deposited dose in contralateral lung in all patients.

The results of a multi-institutional randomized trial, RTOG1308 [RTOG, 2014], comparing photons and particle therapy in treating NSCLC, will have an important impact on treating NSCLC. The trial started in 2014. 



\section{Conclusion and Outlook}

To ensure the tumor will receive the planned dose and the dose to the normal tissue will not exceed the prescribed limits, it is imperative to include a time-resolved (4D) dose calculation in a scanned carbon-ions (PT) treatment planning. A 4D dose calculation is based on the deformable image registration (DIR). DIR is a complex problem and hence prone to errors. Since any errors in DIR may significantly affect the 4D dose calculation, a DIR quality assurance must be conducted before using DIR in the PT treatment planning for lung cancer.

To make PT treatment plans more robust against the uncertainties, there is an emerging trend of including uncertainties in the optimization process itself, the so-called robust optimization. A standard geometrical margin definition inherited from the photon radiotherapy may be inadequate in PT. The robust optimization, however, can substantially improve the treatment plans [Chen et al., 2012]. Furthermore, the robustness optimization is now computationally possible even for a 4D optimization [Liu et al., 2016b], bringing treatment of lung cancer patients with PT closer to reality. Future studies on treating lung cancer with PT should definitely include a robust 4D dose optimization.

The uncertainties in the radiation treatment could drastically be reduced by employing real-time imaging at the moment of irradiation. This was achieved by merging MRI and photon Linac, a promising new technology in the field of radio therapy. The application is being realized in a few clinics worldwide and first patients will be treated in the near future [Lagendijk et al., 2016]. Although the challenge of combining PT with MRI may be greater for particles than for photons, studies have shown the feasibility of a such combination [Hartman et al., 2015]. In addition, it was shown that particles could be used for imaging purposes as well, opening a wide field of new possibilities for PT [Prall et al., 2016a]. 
Based on the results of this study, PT should be considered as a treatment modality for the non-small cell lung cancer. PT not only delivers the same dose to the tumors as the tumors as the state of the art photon therapy (SBRT), but also tremendously reduces the normal tissue irradiation.

PT would be especially beneficial for patients with large tumors or with multiple disease sites, because the dose bath is much smaller than with SBRT. This could play a crucial role in some patients in which PT could deliver the full ablative dose in a single fraction, whereas SBRT is limited due to over irradiating normal tissue.

Patients with an advanced stage of lung cancer have an extremely poor prognosis, with a median survival rate of only nine months. Currently the best treatment, a combination of chemotherapy and SBRT, is able to extend the patient survival rate to 20 months. However, every tenth patient will die due to treatment-related effects. We have shown that PT has the potential to significantly reduce the radiation-related side effects and hence tremendously improve the survival rate. PT should therefore definitely be further investigated as a lung cancer treatment modality, especially for an advanced stage disease. 


\section{A Appendix of Chapter 2}

\section{Contents}

A.1. Patient hierarchy $\ldots \ldots \ldots \ldots \ldots \ldots \ldots \ldots \ldots \ldots \ldots \ldots$

\section{A.1 Patient hierarchy}

Patient hierarchy follows a subject hierarchy principle in Slicer. It was designed for a clear overview of the registration process, DIRQA and all resulting files. Another reason is to track DIR and DIRQA in case if they are interrupted by Slicer crash. DIR and DIRQA files can be quite large and can cause Slicer to run out of memory. With patient hierarchy Slicer is able to continue work from where it was interrupted rather than starting anew.

There are several levels in patient hierarchy. Each level also has different attributes, where details regarding each level can be written.

- Level 1: Patient name - separates different patients.

- Level 2: Registration node - separates between different registrations, e.g. between different imaging modalities or between 4D-CT phases.

Attributes:

- The file directory of images, vector fields and registration quality files.

- Number of phases to be registered.

- Reference phase

- Level 3: Registration set - specific registration phase. Registration is done between all phases and the reference one. There have to be at least two phases

- Level 4: Node - can be either an image, a vector field, an inverse vector field or any of DIRQA nodes (see Section 2.2.3).

Attributes:

- Exact file paths for specific node.

- Statistical analysis if node is absolute difference, Jacobian or inverse consistency (see Section 2.2.3). 
The patient hierarchy can be constructed in two ways. The first option is to manually create the whole patient hierarchy, from top to bottom level, with necessary attributes. Second option is to use an automatic script to look for files on hard drive and create corresponding levels. The second option is possible only by using proper naming conventions for file names and locations. 


\section{B Appendix of Chapter 3}

\section{B.1 Organs at risk dose limits}

OAR dose limits used in SBRT and PT treatment planning for different fractionation schemes are shown in Table B.1. Two limits were used. The first limitation was a maximum dose to single voxel $D_{\text {Max }}$ and the second a maximum dose deposited to a specific OAR volume $D_{\text {Threshold }}$.

Table B.1.: Dose constraints for various OARs for 1, 2 and 3 fractions, denoted as respective numbers. Limits were used in SBRT and PT treatment planning. Data taken from [Benedict et al., 2010]

\begin{tabular}{|c|c|c|c|c|c|c|c|}
\hline & Critical & \multicolumn{4}{|c|}{ Threshold dose (Gy) } & \multicolumn{3}{|c|}{ Maximum point dose(Gy) } \\
Organ & volume (cc) & 1 & 2 & 3 & 1 & 2 & 3 \\
\hline heart & 15 & 16 & 22 & 24 & 30 & 32 & 38 \\
spinal cord & 0.35 & 10 & 14 & 18 & 21.9 & 23 & 30 \\
smaller airways & 0.5 & 12.4 & 13.3 & 18.9 & 23.1 & 21 & 33 \\
esophagus & 5 & 11.9 & 15.4 & 17.7 & 25.2 & 19.5 & 35 \\
trachea & 4 & 10.5 & 20.2 & 15 & 30 & 16.5 & 40 \\
aorta & 10 & 31 & 37 & 39 & 45 & 47 & 53 \\
stomach & 10 & 11.2 & 12.4 & 16.5 & 22.2 & 18 & 32 \\
\hline \hline
\end{tabular}




\section{B.2 Target coverage}

Target coverage for SBRT and PT. For PT target coverage was calculated in 3D case on state 0 and 5 (3D-Dose ${ }_{0} \% 3 \mathrm{D}$-Dose ${ }_{5} 0 \%$; ) 4D doses were calculated for two different breathing periods (3.6 s and 5s) and for two starting phases $\left(0^{\circ}\right.$ and $\left.90^{\circ}\right)$. 4D doses were calculated without motion compensation (4D-Dose interplay ) and with rescanning as a motion mitigation technique (4D-Dose rescanning ).

Table B.2.: Target coverage for PT and SBRT as CTV $D_{99 \%}$. PT was calculated two static cases (3D, state 0 and 5) and 8 4D cases - two breathing periods: 3.6 and $5 \mathrm{~s}$ (Per 3s and Per 5s), two starting phases: $0^{\circ}$ and $90^{\circ}$ (Ph0 and Ph90) and without rescanning (interplay) and with rescanning. All values are displayed as percentage of the planned dose.

\begin{tabular}{|c|c|c|c|c|c|c|c|c|c|c|c|}
\hline \multirow{3}{*}{$\begin{array}{l}\text { Tar- } \\
\text { get }\end{array}$} & \multicolumn{2}{|c|}{$3 \mathrm{D}$} & \multicolumn{4}{|c|}{ 4D interplay } & \multicolumn{4}{|c|}{ 4D rescan } & \multirow{3}{*}{ SBRT } \\
\hline & \multirow{2}{*}{$\begin{array}{c}\text { State } \\
0\end{array}$} & \multirow{2}{*}{$\begin{array}{c}\text { State } \\
5\end{array}$} & \multicolumn{2}{|c|}{ Period 3.6s } & \multicolumn{2}{|c|}{ Period 5s } & \multicolumn{2}{|c|}{ Period $3.6 \mathrm{~s}$} & \multicolumn{2}{|c|}{ Period 5s } & \\
\hline & & & Ph0 & Ph90 & Ph0 & Ph90 & Ph0 & $\mathrm{Ph} 90$ & Ph0 & $\mathrm{Ph} 90$ & \\
\hline 1 & 101.04 & 101.04 & 98.96 & 98.96 & 98.96 & 100.0 & 101.04 & 101.04 & 101.04 & 101.04 & 03.13 \\
\hline 2 & 2.08 & 2.08 & 1.04 & 98.96 & 0.0 & 2 & 4 & 102.08 & 02.08 & 4 & 01. \\
\hline 3 & 4.17 & 105.21 & 1( & 97.92 & 5.83 & .92 & 7 & 106.25 & 103.13 & 107. & 01.0 \\
\hline 4 & 0.0 & 10 & 86 & 100.0 & 92.86 & 2.86 & 6.43 & 103.57 & 00.0 & & 100.0 \\
\hline 5 & 1.04 & 101.0 & 101.04 & 101.04 & 101.04 & 101 & 101.04 & 101.04 & 101.04 & & 101.04 \\
\hline 6 & 2.08 & 103.13 & 100.0 & 100.0 & 98.96 & 0.0 & 100.0 & 100.0 & 101.04 & & 101.04 \\
\hline 7 & 2.08 & 10 & 97.92 & 95.83 & 92.71 & 4.79 & 101.04 & 100.0 & 101.04 & & 100.0 \\
\hline 8 & 2.08 & 102.08 & 100.0 & 100.0 & 98.96 & 7.92 & 102.08 & 101.04 & 102.08 & .08 & 01.04 \\
\hline 9 & 1.04 & 102.08 & 98.96 & 98.96 & 96.88 & 97.92 & 101.04 & 100.0 & 101.04 & 101.04 & 01.04 \\
\hline 10 & 0.0 & 100.0 & 98.96 & 100.0 & 98.96 & 100.0 & 101.04 & 100.0 & 101.04 & & 102.08 \\
\hline 11 & 2.08 & 10 & 98.96 & 98.96 & 98.96 & 97.92 & 101.04 & 101.04 & 101.04 & .04 & 100.0 \\
\hline 12 & 2.08 & 10 & 98.96 & 100.0 & 98.96 & 7.92 & 101.04 & 102.08 & 101.04 & & 100.0 \\
\hline 13 & 2.08 & 102.08 & 97.92 & 97.92 & 96.88 & 96.88 & 102.08 & 100.0 & 101.04 & 101.04 & 106.25 \\
\hline 14 & 1.04 & 101.04 & 100.0 & 100.0 & 98.96 & 98.96 & 101.04 & 101.04 & 101.04 & 101.04 & 03.13 \\
\hline 15 & 1.04 & 102.08 & 100.0 & 97.92 & 98.96 & 95.83 & 101.04 & 100.0 & 101.04 & 101.04 & 104.17 \\
\hline 16 & 1.04 & 100.0 & 95.83 & 96.88 & 97.92 & 96.88 & 100.0 & 98.96 & 101.04 & 100.0 & 107.29 \\
\hline 17 & 2.08 & 102.08 & 100.0 & 100.0 & 98.96 & 96.88 & 101.04 & 102.08 & 102.08 & 101.04 & 08.33 \\
\hline 18 & 1.04 & 101.04 & 95.83 & 100.0 & 96.88 & 94.79 & 101.04 & 101.04 & 101.04 & 101.04 & 101.04 \\
\hline 19 & 101.04 & 98.96 & 98.96 & 96.88 & 97.92 & 97.92 & 98.96 & 98.96 & 100.0 & 98.96 & 104.17 \\
\hline 20 & 82.29 & 81.25 & 85.42 & 85.42 & 83.33 & & 85.42 & 86.46 & 86.46 & 85.42 & 70.83 \\
\hline 21 & 101.04 & 101.04 & 97.92 & 96.88 & 94.79 & 96.88 & 98.96 & 102.08 & 100.0 & 101.04 & 102.08 \\
\hline 22 & 1.04 & 101.04 & 101.04 & 101.04 & 101.04 & 100.0 & 101.04 & 101.04 & 101.04 & 101.04 & 101.04 \\
\hline 23 & 102.08 & 102.08 & 98.96 & 98.96 & 98.96 & 98.96 & 102.08 & 101.04 & 101.04 & 101.04 & 101.04 \\
\hline 24 & 100.0 & 100.0 & 95.83 & 97.92 & 96.88 & 96.88 & 100.0 & 98.96 & 100.0 & 100.0 & 0.0 \\
\hline 25 & 101.04 & 102.08 & 98.96 & 98.96 & 96.88 & 97.92 & 101.04 & 100.0 & 101.04 & 101.04 & 101.04 \\
\hline 26 & 101.04 & 102.08 & 100.0 & 100.0 & 98.96 & 98.96 & 100.0 & 101.04 & 101.04 & 101.04 & 101.04 \\
\hline 27 & 3.13 & 103.13 & 102.08 & 100.0 & 101.04 & 101.04 & 102.08 & 101.04 & 103.13 & 102.08 & 101.04 \\
\hline
\end{tabular}




\section{B.3 Dose to organs at risk}

Details on the dose to OARs for PT and SBRT will be given here. Various OAR's $D_{\text {Max }}, D_{\text {Threshold }}$ and $D_{\text {Mean }}$ will be given here for all patients used in the study.

Table B.3.: SBRT $D_{\operatorname{Max}}$ for various OARs. Values are in Gy.

\begin{tabular}{|c|c|c|c|c|c|c|c|c|}
\hline $\begin{array}{c}\text { Patient } \\
\mathrm{Nr}\end{array}$ & Heart & $\begin{array}{c}\text { Spinal } \\
\text { cord }\end{array}$ & $\begin{array}{c}\text { Smaller } \\
\text { airways }\end{array}$ & Esophagus & Trachea & Aorta & $\begin{array}{c}\text { Left } \\
\text { lung }\end{array}$ & $\begin{array}{c}\text { Right } \\
\text { lung }\end{array}$ \\
\hline \hline 1 & 17.0 & 4.25 & 16.0 & 4.75 & 0.5 & 6.25 & 26.75 & 4.5 \\
2 & 0 & 2.75 & 16.75 & 3.5 & 2.75 & 5.5 & 26.75 & 2.5 \\
3 & 7.5 & 8.5 & 17.5 & 7.0 & 0.25 & 7.75 & 10.0 & 26.5 \\
4 & 0.25 & 5.5 & 10.25 & 5.5 & 0 & 5.0 & 26.25 & 3.5 \\
5 & 8.25 & 10.25 & 12.75 & 9.75 & 0 & 26.25 & 26.25 & 9.25 \\
6 & 6.0 & 2.5 & 23.75 & 3.5 & 0 & 4.5 & 26.25 & 3.5 \\
7 & 0.25 & 7.0 & 19.25 & 6.0 & 9.75 & 8.25 & 4.75 & 25.75 \\
8 & 12.5 & 8.75 & 13.75 & 14.0 & 3.5 & 24.75 & 26.75 & 11.5 \\
9 & 11.25 & 9.75 & 22.75 & 11.0 & 0.25 & 19.25 & 25.75 & 26.0 \\
10 & 15.0 & 9.25 & 13.0 & 10.75 & 0 & 27.25 & 27.75 & 27.5 \\
11 & 12.0 & 2.75 & 0.25 & 2.25 & 0 & 4.25 & 26.25 & 1.75 \\
12 & 0.0 & 4.75 & 0.0 & 7.0 & 7.75 & 9.75 & 25.75 & 5.25 \\
13 & 3.25 & 6.25 & 11.0 & 4.5 & 0 & 3.5 & 4.25 & 26.5 \\
14 & 0.0 & 3.75 & 9.25 & 4.0 & 4.75 & 5.5 & 2.25 & 26.0 \\
15 & 0 & 3.75 & 0 & 5.75 & 0 & 0 & 26.0 & 5.0 \\
16 & 2.5 & 2.0 & 0 & 3.75 & 4.75 & 18.0 & 10.0 & 26.25 \\
17 & 0.25 & 8.25 & 0 & 11.5 & 7.25 & 26.25 & 26.25 & 6.75 \\
18 & 0 & 2.5 & 0 & 3.5 & 2.25 & 0.0 & 2.25 & 26.0 \\
19 & 0 & 8.5 & 0.75 & 6.0 & 4.25 & 22.75 & 26.0 & 5.0 \\
\hline \hline
\end{tabular}


Table B.4.: SBRT $D_{\text {Threshold }}$ for various OARs. Values are in Gy.

\begin{tabular}{|c|c|c|c|c|c|c|c|c|}
\hline $\begin{array}{c}\text { Patient } \\
\mathrm{Nr}\end{array}$ & Heart & $\begin{array}{c}\text { Spinal } \\
\text { cord }\end{array}$ & $\begin{array}{c}\text { Smaller } \\
\text { airways }\end{array}$ & Esophagus & Trachea & Aorta & $\begin{array}{c}\text { Left } \\
\text { lung }\end{array}$ & $\begin{array}{c}\text { Right } \\
\text { lung }\end{array}$ \\
\hline \hline 1 & 9.75 & 3.5 & 7.87 & 3.75 & 0.0 & 4.75 & 0.75 & 0.25 \\
2 & 0 & 2.0 & 10.0 & 1.5 & 1.67 & 3.25 & 0.0 & 0.0 \\
3 & 6.0 & 7.5 & 5.25 & 3.25 & 0.0 & 5.67 & 0.0 & 0.0 \\
4 & 0.0 & 4.75 & 3.75 & 2.75 & 0 & 3.5 & 0.0 & 0.0 \\
5 & 6.0 & 8.25 & 0.96 & 1.0 & 0 & 11.75 & 0.0 & 0.0 \\
6 & 4.0 & 2.25 & 5.0 & 1.11 & 0 & 1.75 & 0.0 & 0.0 \\
7 & 0.0 & 6.5 & 11.5 & 3.25 & 5.5 & 6.0 & 0.0 & 0.0 \\
8 & 9.75 & 7.25 & 10.75 & 11.0 & 2.62 & 18.25 & 1.75 & 1.69 \\
9 & 8.5 & 8.5 & 12.25 & 6.75 & 0.0 & 12.5 & 0.0 & 0.0 \\
10 & 10.25 & 8.5 & 7.25 & 6.25 & 0 & 10.5 & 0.0 & 0.25 \\
11 & 5.75 & 2.5 & 0.33 & 0.25 & 0 & 1.5 & 0.0 & 0.0 \\
12 & 0.0 & 4.0 & 0.0 & 0.5 & 5.5 & 4.5 & 0.0 & 0.0 \\
13 & 1.75 & 5.5 & 4.25 & 3.25 & 0 & 2.25 & 0.0 & 0.0 \\
14 & 0.0 & 3.5 & 0.42 & 2.0 & 2.25 & 2.75 & 0.0 & 0.0 \\
15 & 0 & 3.25 & 0 & 1.25 & 0 & 0 & 0.0 & 0.0 \\
16 & 0.0 & 1.5 & 0 & 0.25 & 2.82 & 5.25 & 0.0 & 0.0 \\
17 & 0.0 & 7.0 & 0 & 3.5 & 4.9 & 6.25 & 0.0 & 0.0 \\
18 & 0 & 2.25 & 0 & 1.87 & 1.44 & 0.0 & 0.0 & 0.0 \\
19 & 0 & 7.5 & 0.0 & 1.75 & 2.25 & 5.0 & 0.0 & 0.0 \\
\hline \hline
\end{tabular}


Table B.5.: SBRT $D_{\text {Mean }}$ for various OARs. Values are in Gy.

\begin{tabular}{|c|c|c|c|c|c|c|c|c|}
\hline $\begin{array}{c}\text { Patient } \\
\mathrm{Nr}\end{array}$ & Heart & $\begin{array}{c}\text { Spinal } \\
\text { cord }\end{array}$ & $\begin{array}{c}\text { Smaller } \\
\text { airways }\end{array}$ & Esophagus & Trachea & Aorta & $\begin{array}{c}\text { Left } \\
\text { lung }\end{array}$ & $\begin{array}{c}\text { Right } \\
\text { lung }\end{array}$ \\
\hline \hline 1 & 1.96 & 0.52 & 6.5 & 0.92 & 0.11 & 1.31 & 3.88 & 0.7 \\
2 & 0 & 0.28 & 5.53 & 0.43 & 0.41 & 0.97 & 1.46 & 0.23 \\
3 & 2.48 & 2.27 & 2.83 & 4.08 & 0.06 & 2.43 & 0.9 & 2.9 \\
4 & 0.06 & 0.81 & 3.02 & 0.77 & 0 & 1.08 & 1.88 & 0.49 \\
5 & 1.49 & 0.66 & 3.8 & 1.18 & 0 & 2.38 & 1.79 & 0.61 \\
6 & 1.34 & 0.19 & 6.1 & 1.22 & 0 & 1.57 & 1.3 & 0.2 \\
7 & 0.07 & 0.7 & 6.08 & 1.07 & 1.81 & 1.65 & 0.69 & 2.88 \\
8 & 6.4 & 2.02 & 6.33 & 4.65 & 0.92 & 5.75 & 8.82 & 2.24 \\
9 & 3.57 & 1.66 & 2.57 & 1.8 & 0.1 & 1.68 & 3.05 & 2.95 \\
10 & 4.72 & 1.2 & 2.65 & 2.97 & 0 & 3.45 & 5.4 & 5.15 \\
11 & 1.52 & 0.38 & 0.17 & 0.26 & 0 & 0.3 & 1.65 & 0.2 \\
12 & 0.02 & 0.26 & 0.03 & 0.7 & 1.85 & 0.4 & 1.44 & 0.3 \\
13 & 0.77 & 1.18 & 2.16 & 1.68 & 0 & 0.48 & 0.6 & 3.62 \\
14 & 0.03 & 0.27 & 0.78 & 0.55 & 1.01 & 0.76 & 0.22 & 1.52 \\
15 & 0 & 0.49 & 0 & 1.12 & 0 & 0 & 0.7 & 0.09 \\
16 & 0.06 & 0.25 & 0 & 0.49 & 1.14 & 1.49 & 0.37 & 2.29 \\
17 & 0.08 & 1.69 & 0 & 1.15 & 1.94 & 1.51 & 2.39 & 0.4 \\
18 & 0 & 0.27 & 0 & 0.58 & 0.51 & 0.04 & 0.06 & 0.38 \\
19 & 0 & 0.75 & 0.13 & 0.62 & 1.0 & 0.73 & 1.4 & 0.28 \\
\hline \hline
\end{tabular}


Table B.6.: PT $D_{\text {Max }}$ for various OARs. Values are in Gy.

\begin{tabular}{|c|c|c|c|c|c|c|c|c|}
\hline $\begin{array}{c}\text { Patient } \\
\mathrm{Nr}\end{array}$ & Heart & $\begin{array}{c}\text { Spinal } \\
\text { cord }\end{array}$ & $\begin{array}{c}\text { Smaller } \\
\text { airways }\end{array}$ & Esophagus & Trachea & Aorta & $\begin{array}{c}\text { Left } \\
\text { lung }\end{array}$ & $\begin{array}{c}\text { Right } \\
\text { lung }\end{array}$ \\
\hline \hline 1 & 11.75 & 0.0 & 24.0 & 0.25 & 0.0 & 3.75 & 26.0 & 0.75 \\
2 & 0 & 0.0 & 25.25 & 0.0 & 0.0 & 0.25 & 26.5 & 0.0 \\
3 & 7.5 & 0.25 & 9.75 & 7.25 & 0.0 & 7.75 & 7.5 & 27.25 \\
4 & 0.0 & 3.25 & 13.5 & 0.25 & 0 & 4.0 & 25.5 & 1.0 \\
5 & 10.0 & 6.0 & 10.25 & 3.75 & 0 & 26.75 & 26.75 & 0.25 \\
6 & 0.0 & 0.0 & 25.25 & 0.0 & 0 & 0.0 & 26.5 & 0.0 \\
7 & 0.0 & 7.0 & 22.25 & 0.0 & 0.0 & 0.0 & 0.0 & 26.25 \\
8 & 6.5 & 7.75 & 15.5 & 1.0 & 1.0 & 20.75 & 31.5 & 8.25 \\
9 & 0.0 & 0.5 & 6.25 & 0.0 & 0.0 & 4.25 & 25.75 & 25.5 \\
10 & 13.5 & 0.0 & 16.0 & 0.25 & 0 & 25.25 & 26.75 & 26.0 \\
11 & 14.5 & 0.0 & 0.0 & 0.0 & 0 & 0.0 & 26.25 & 0.0 \\
12 & 0.0 & 0.0 & 0.0 & 0.0 & 0.0 & 0.25 & 26.25 & 0.0 \\
13 & 0.0 & 0.0 & 0.0 & 0.0 & 0 & 0.0 & 0.0 & 26.5 \\
14 & 0.0 & 0.0 & 8.0 & 0.0 & 0.0 & 0.0 & 0.0 & 25.5 \\
15 & 0 & 0.0 & 0 & 0.0 & 0 & 0 & 25.75 & 0.0 \\
16 & 0.25 & 0.0 & 0 & 0.0 & 0.0 & 18.25 & 0.75 & 26.0 \\
17 & 0.0 & 0.5 & 0 & 9.0 & 0.5 & 25.75 & 26.0 & 0.0 \\
18 & 0 & 0.0 & 0 & 0.0 & 0.0 & 0.0 & 0.0 & 25.75 \\
19 & 0 & 0.25 & 0.25 & 0.0 & 0.0 & 17.75 & 26.5 & 0.0 \\
\hline \hline
\end{tabular}


Table B.7.: PT $D_{\text {Threshold }}$ for various OARs. Values are in Gy.

\begin{tabular}{|c|c|c|c|c|c|c|c|c|}
\hline $\begin{array}{c}\text { Patient } \\
\mathrm{Nr}\end{array}$ & Heart & $\begin{array}{c}\text { Spinal } \\
\text { cord }\end{array}$ & $\begin{array}{c}\text { Smaller } \\
\text { airways }\end{array}$ & Esophagus & Trachea & Aorta & $\begin{array}{c}\text { Left } \\
\text { lung }\end{array}$ & $\begin{array}{c}\text { Right } \\
\text { lung }\end{array}$ \\
\hline \hline 1 & 8.25 & 0.0 & 7.75 & 0.0 & 0.0 & 0.75 & 0.0 & 0.0 \\
2 & 0 & 0.0 & 13.75 & 0.0 & 0.0 & 0.0 & 0.0 & 0.0 \\
3 & 3.5 & 0.0 & 1.0 & 0.0 & 0.0 & 0.42 & 0.0 & 0.0 \\
4 & 0.0 & 2.0 & 5.25 & 0.0 & 0 & 0.75 & 0.0 & 0.0 \\
5 & 2.5 & 1.75 & 0.0 & 0.0 & 0 & 14.0 & 0.0 & 0.0 \\
6 & 0.0 & 0.0 & 0.0 & 0.0 & 0 & 0.0 & 0.0 & 0.0 \\
7 & 0.0 & 3.0 & 10.25 & 0.0 & 0.0 & 0.0 & 0.0 & 0.0 \\
8 & 1.91 & 5.5 & 11.0 & 0.25 & 0.0 & 7.25 & 0.0 & 0.0 \\
9 & 0.0 & 32.0 & 0.11 & 0.0 & 0.0 & 0.25 & 0.0 & 0.0 \\
10 & 10.25 & 0.0 & 0.5 & 0.0 & 0 & 3.75 & 0.0 & 0.0 \\
11 & 0.25 & 0.0 & 0.0 & 0.0 & 0 & 0.0 & 0.0 & 0.0 \\
12 & 0.0 & 0.0 & 0.0 & 0.0 & 0.0 & 0.0 & 0.0 & 0.0 \\
13 & 0.0 & 0.0 & 0.0 & 0.0 & 0 & 0.0 & 0.0 & 0.0 \\
14 & 0.0 & 0.0 & 0.0 & 0.0 & 0.0 & 0.0 & 0.0 & 0.0 \\
15 & 0 & 0.0 & 0 & 0.0 & 0 & 0 & 0.0 & 0.0 \\
16 & 0.0 & 0.0 & 0 & 0.0 & 0.0 & 0.25 & 0.0 & 0.0 \\
17 & 0.0 & 0.25 & 0 & 0.0 & 0.0 & 5.5 & 0.0 & 0.0 \\
18 & 0 & 0.0 & 0 & 0.0 & 0.0 & 0.0 & 0.0 & 0.0 \\
19 & 0 & 0.0 & 0.0 & 0.0 & 0.0 & 0.25 & 0.0 & 0.0 \\
\hline \hline
\end{tabular}


Table B.8.: PT $D_{\text {Mean }}$ for various OARs. Values are in Gy.

\begin{tabular}{|c|c|c|c|c|c|c|c|c|}
\hline $\begin{array}{c}\text { Patient } \\
\mathrm{Nr}\end{array}$ & Heart & $\begin{array}{c}\text { Spinal } \\
\text { cord }\end{array}$ & $\begin{array}{c}\text { Smaller } \\
\text { airways }\end{array}$ & Esophagus & Trachea & Aorta & $\begin{array}{c}\text { Left } \\
\text { lung }\end{array}$ & $\begin{array}{c}\text { Right } \\
\text { lung }\end{array}$ \\
\hline \hline 1 & 0.53 & 0.0 & 3.67 & 0.01 & 0.0 & 0.08 & 3.46 & 0.03 \\
2 & 0 & 0.0 & 4.32 & 0.0 & 0.0 & 0.01 & 1.7 & 0.0 \\
3 & 0.49 & 0.02 & 0.56 & 0.91 & 0.0 & 0.51 & 0.12 & 2.5 \\
4 & 0.0 & 0.17 & 3.58 & 0.0 & 0 & 0.09 & 1.35 & 0.06 \\
5 & 0.45 & 0.03 & 0.47 & 0.22 & 0 & 2.24 & 0.61 & 0.0 \\
6 & 0.0 & 0.0 & 2.96 & 0.0 & 0 & 0.0 & 1.58 & 0.0 \\
7 & 0.0 & 0.11 & 2.11 & 0.0 & 0.0 & 0.0 & 0.0 & 2.97 \\
8 & 0.6 & 0.68 & 2.25 & 0.06 & 0.06 & 1.08 & 6.0 & 0.36 \\
9 & 0.0 & 0.05 & 0.06 & 0.01 & 0.0 & 0.03 & 1.71 & 1.24 \\
10 & 1.16 & 0.0 & 0.5 & 0.01 & 0 & 0.68 & 3.45 & 3.55 \\
11 & 0.06 & 0.0 & 0.0 & 0.0 & 0 & 0.0 & 2.26 & 0.0 \\
12 & 0.0 & 0.0 & 0.0 & 0.0 & 0.0 & 0.0 & 1.03 & 0.0 \\
13 & 0.0 & 0.01 & 0.01 & 0.0 & 0 & 0.0 & 0.0 & 2.14 \\
14 & 0.0 & 0.0 & 0.28 & 0.0 & 0.0 & 0.0 & 0.0 & 1.88 \\
15 & 0 & 0.01 & 0 & 0.01 & 0 & 0 & 0.9 & 0.0 \\
16 & 0.0 & 0.0 & 0 & 0.0 & 0.0 & 0.16 & 0.0 & 1.88 \\
17 & 0.0 & 0.03 & 0 & 0.15 & 0.03 & 1.29 & 2.55 & 0.0 \\
18 & 0 & 0.0 & 0 & 0.0 & 0.0 & 0.0 & 0.0 & 0.4 \\
19 & 0 & 0.01 & 0.01 & 0.0 & 0.0 & 0.07 & 1.49 & 0.0 \\
\hline \hline
\end{tabular}




\section{Appendix to Chapter 4}

\section{C.1 Target coverage}

Target coverage is displayed as CTV $D_{99 \%}$ in Table C.1. It was calculated for ITV, 4Dopt and SBRT. For ITV and 4Dopt 4 different breathing periods were used.

Table C.1.: Target coverage for ITV, 4DOpt and SBRT as CTV $D_{99 \%}$. ITV and 4Dopt used two breathing periods: 3.6 and $5 \mathrm{~s}$ (Per $3 \mathrm{~s}$ and Per 5s) and two starting phases: $0^{\circ}$ and $90^{\circ}$ (Ph0 and Ph90). All values are displayed as percentage of the planned dose.

\begin{tabular}{|c|c|c|c|c|c|c|c|c|c|}
\hline \multirow{3}{*}{ Target } & \multicolumn{4}{|c|}{ ITV } & \multicolumn{4}{|c|}{ 4Dopt } & \multirow{3}{*}{ SBRT } \\
\hline & \multicolumn{2}{|c|}{ Per 3.6s } & \multicolumn{2}{|c|}{ Per 5s } & \multicolumn{2}{|c|}{ Per 3600s } & \multicolumn{2}{|c|}{ Per 5000s } & \\
\hline & $\mathrm{Ph}=0$ & $\mathrm{Ph}=90$ & Ph0 & Ph90 & Ph0 & Ph90 & Ph0 & Ph90 & \\
\hline $1 \mathrm{a}$ & 101.04 & 101.04 & 101.04 & 101.04 & 101.04 & 101.04 & 101.04 & 101.04 & 100.0 \\
\hline $1 \mathrm{~b}$ & 102.08 & 101.04 & 101.04 & 101.04 & 101.04 & 101.04 & 101.04 & 101.04 & 100.0 \\
\hline $2 a$ & 101.04 & 101.04 & 102.08 & 101.04 & 101.04 & 98.96 & 98.96 & 102.08 & 106.25 \\
\hline $2 b$ & 102.08 & 102.08 & 102.08 & 102.08 & 102.08 & 102.08 & 102.08 & 102.08 & 103.13 \\
\hline $2 c$ & 101.04 & 101.04 & 101.04 & 100.0 & 101.04 & 102.08 & 102.08 & 101.04 & 104.17 \\
\hline $2 d$ & 102.08 & 102.08 & 102.08 & 101.04 & 102.08 & 102.08 & 102.08 & 102.08 & 107.29 \\
\hline $2 \mathrm{e}$ & 101.04 & 101.04 & 101.04 & 101.04 & 101.04 & 101.04 & 101.04 & 102.08 & 108.33 \\
\hline $3 a$ & 101.04 & 101.04 & 101.04 & 101.04 & 101.04 & 101.04 & 101.04 & 101.04 & 101.04 \\
\hline $3 b$ & 97.92 & 98.96 & 98.96 & 97.92 & 97.92 & 97.92 & 97.92 & 97.92 & 102.08 \\
\hline $4 a$ & 69.44 & 63.89 & 65.74 & 64.81 & 72.22 & 69.44 & 68.52 & 71.3 & 66.67 \\
\hline $4 \mathrm{~b}$ & 100.0 & 102.08 & 100.0 & 102.08 & 101.04 & 100.0 & 102.08 & 100.0 & 103.13 \\
\hline $5 a$ & 98.96 & 100.0 & 101.04 & 100.0 & 100.0 & 100.0 & 100.0 & 100.0 & 101.04 \\
\hline $5 b$ & 102.08 & 102.08 & 100.0 & 101.04 & 97.92 & 97.92 & 98.96 & 96.88 & 101.04 \\
\hline $5 c$ & 96.88 & 94.79 & 94.79 & 95.83 & 92.71 & 94.79 & 93.75 & 94.79 & 98.96 \\
\hline $5 d$ & 98.96 & 98.96 & 98.96 & 97.92 & 100.0 & 98.96 & 98.96 & 100.0 & 94.79 \\
\hline $6 a$ & 88.54 & 89.58 & 88.54 & 90.63 & 85.42 & 87.5 & 85.42 & 85.42 & 69.79 \\
\hline $6 \mathrm{~b}$ & 78.13 & 79.17 & 77.08 & 79.17 & 72.92 & 71.88 & 71.88 & 72.92 & 69.79 \\
\hline $7 a$ & 102.08 & 102.08 & 102.08 & 102.08 & 98.96 & 98.96 & 98.96 & 98.96 & 101.04 \\
\hline $7 \mathrm{~b}$ & 82.14 & 85.71 & 82.14 & 85.71 & 75.0 & 75.0 & 75.0 & 75.0 & 100.0 \\
\hline $8 a$ & 100.0 & 100.93 & 100.0 & 100.0 & 100.0 & 99.07 & 99.07 & 100.93 & 105.56 \\
\hline $8 b$ & 101.25 & 101.25 & 100.0 & 102.5 & 100.0 & 100.0 & 100.0 & 101.25 & 105.0 \\
\hline $8 c$ & 100.0 & 100.0 & 100.0 & 99.07 & 97.22 & 99.07 & 100.0 & 100.0 & 106.48 \\
\hline $8 d$ & 102.27 & 102.27 & 102.27 & 102.27 & 90.91 & 89.77 & 89.77 & 89.77 & 102.27 \\
\hline $8 e$ & 102.5 & 102.5 & 102.5 & 102.5 & 91.25 & 92.5 & 91.25 & 92.5 & 101.25 \\
\hline
\end{tabular}




\section{C.2 Dose to organs at risk}

Details on the dose to OARs for ITV, 4Dopt and SBRT will be given here. Various OAR's $D_{\text {Max }}$, $D_{\text {Threshold }}$ and $D_{\text {Mean }}$ will be for all patients used in the study.

Table C.2.: ITV $D_{\text {Max }}$ for various OARs. Values are in Gy.

\begin{tabular}{c|c|c|c|c|c|c|c|c}
$\begin{array}{c}\text { Patient } \\
\mathrm{Nr}\end{array}$ & Heart & $\begin{array}{c}\text { Spinal } \\
\text { cord }\end{array}$ & $\begin{array}{c}\text { Smaller } \\
\text { airways }\end{array}$ & Esophagus & Trachea & Aorta & $\begin{array}{c}\text { Left } \\
\text { lung }\end{array}$ & $\begin{array}{c}\text { Right } \\
\text { lung }\end{array}$ \\
\hline \hline 1 & 0.0 & 0.0 & 7.0 & 0.0 & 0.0 & 3.0 & 26.0 & 26.0 \\
2 & 11.0 & 0.0 & 12.0 & 0.0 & 0.0 & 23.0 & 26.0 & 26.0 \\
3 & 5.0 & 7.0 & 14.0 & 1.0 & 1.0 & 20.0 & 26.0 & 7.0 \\
4 & 25.0 & 8.0 & 23.0 & 20.0 & 3.0 & 4.0 & 26.0 & 29.0 \\
5 & 22.0 & 1.0 & 0.0 & 1.0 & 0.0 & 3.0 & 29.0 & 1.0 \\
6 & 17.0 & 5.0 & 17.0 & 2.0 & 0.0 & 8.0 & 27.0 & 29.0 \\
7 & 7.0 & 0.0 & 9.0 & 7.0 & 0.0 & 8.0 & 9.0 & 26.0 \\
8 & 28.0 & 5.0 & 21.0 & 7.0 & 7.0 & 28.0 & 30.0 & 25.0 \\
\hline \hline
\end{tabular}

Table C.3.: ITV $D_{\text {Threshold }}$ for various OARs. Values are in Gy.

\begin{tabular}{c|c|c|c|c|c|c|c|c}
$\begin{array}{c}\text { Patient } \\
\text { Nr }\end{array}$ & Heart & $\begin{array}{c}\text { Spinal } \\
\text { cord }\end{array}$ & $\begin{array}{c}\text { Smaller } \\
\text { airways }\end{array}$ & Esophagus & Trachea & Aorta & $\begin{array}{c}\text { Left } \\
\text { lung }\end{array}$ & $\begin{array}{c}\text { Right } \\
\text { lung }\end{array}$ \\
\hline \hline 1 & 0.0 & 0.0 & 0.0 & 0.0 & 0.0 & 0.0 & 0.0 & 0.0 \\
2 & 8.0 & 0.0 & 2.0 & 0.0 & 0.0 & 2.0 & 0.0 & 0.0 \\
3 & 2.0 & 5.0 & 8.0 & 0.0 & 0.0 & 6.0 & 0.0 & 0.0 \\
4 & 4.0 & 6.0 & 17.0 & 3.0 & 0.0 & 2.0 & 0.0 & 0.0 \\
5 & 2.0 & 1.0 & 0.0 & 0.0 & 0.0 & 1.0 & 0.0 & 0.0 \\
6 & 4.0 & 3.0 & 12.0 & 1.0 & 0.0 & 2.0 & 0.0 & 0.0 \\
7 & 1.0 & 0.0 & 2.0 & 0.0 & 0.0 & 0.0 & 0.0 & 0.0 \\
8 & 14.0 & 3.0 & 9.0 & 2.0 & 4.0 & 12.0 & 0.0 & 1.0 \\
\hline \hline
\end{tabular}

Table C.4.: ITV $D_{\text {Mean }}$ for various OARs. Values are in Gy.

\begin{tabular}{c|c|c|c|c|c|c|c|c}
$\begin{array}{c}\text { Patient } \\
\mathrm{Nr}\end{array}$ & Heart & $\begin{array}{c}\text { Spinal } \\
\text { cord }\end{array}$ & $\begin{array}{c}\text { Smaller } \\
\text { airways }\end{array}$ & Esophagus & Trachea & Aorta & $\begin{array}{c}\text { Left } \\
\text { lung }\end{array}$ & $\begin{array}{c}\text { Right } \\
\text { lung }\end{array}$ \\
\hline \hline 1 & 0.0 & 0.0 & 0.0 & 0.0 & 0.0 & 0.0 & 1.0 & 1.0 \\
2 & 1.0 & 0.0 & 1.0 & 0.0 & 0.0 & 0.0 & 3.0 & 3.0 \\
3 & 0.0 & 1.0 & 2.0 & 0.0 & 0.0 & 1.0 & 4.0 & 0.0 \\
4 & 1.0 & 1.0 & 6.0 & 1.0 & 0.0 & 0.0 & 2.0 & 1.0 \\
5 & 0.0 & 0.0 & 0.0 & 0.0 & 0.0 & 0.0 & 4.0 & 0.0 \\
6 & 0.0 & 0.0 & 3.0 & 0.0 & 0.0 & 1.0 & 4.0 & 2.0 \\
7 & 0.0 & 0.0 & 1.0 & 1.0 & 0.0 & 0.0 & 0.0 & 2.0 \\
8 & 1.0 & 0.0 & 4.0 & 1.0 & 1.0 & 1.0 & 8.0 & 6.0 \\
\hline \hline
\end{tabular}


Table C.5.: 4Dopt $D_{\text {Max }}$ for various OARs. Values are in Gy.

\begin{tabular}{c|c|c|c|c|c|c|c|c}
$\begin{array}{c}\text { Patient } \\
\text { Nr }\end{array}$ & Heart & $\begin{array}{c}\text { Spinal } \\
\text { cord }\end{array}$ & $\begin{array}{c}\text { Smaller } \\
\text { airways }\end{array}$ & Esophagus & Trachea & Aorta & $\begin{array}{c}\text { Left } \\
\text { lung }\end{array}$ & $\begin{array}{c}\text { Right } \\
\text { lung }\end{array}$ \\
\hline \hline 1 & 0.0 & 0.0 & 7.0 & 0.0 & 0.0 & 3.0 & 26.0 & 26.0 \\
2 & 11.0 & 0.0 & 12.0 & 0.0 & 0.0 & 22.0 & 26.0 & 26.0 \\
3 & 5.0 & 8.0 & 15.0 & 1.0 & 1.0 & 21.0 & 27.0 & 8.0 \\
4 & 28.0 & 8.0 & 22.0 & 25.0 & 1.0 & 6.0 & 26.0 & 29.0 \\
5 & 21.0 & 0.0 & 0.0 & 1.0 & 0.0 & 3.0 & 29.0 & 1.0 \\
6 & 15.0 & 4.0 & 16.0 & 2.0 & 0.0 & 6.0 & 28.0 & 28.0 \\
7 & 7.0 & 0.0 & 8.0 & 7.0 & 0.0 & 7.0 & 8.0 & 26.0 \\
8 & 29.0 & 5.0 & 21.0 & 8.0 & 7.0 & 27.0 & 30.0 & 25.0 \\
\hline \hline
\end{tabular}

Table C.6.: 4 Dopt $D_{\text {Threshold }}$ for various OARs. Values are in Gy.

\begin{tabular}{c|c|c|c|c|c|c|c|c}
$\begin{array}{c}\text { Patient } \\
\text { Nr }\end{array}$ & Heart & $\begin{array}{c}\text { Spinal } \\
\text { cord }\end{array}$ & $\begin{array}{c}\text { Smaller } \\
\text { airways }\end{array}$ & Esophagus & Trachea & Aorta & $\begin{array}{c}\text { Left } \\
\text { lung }\end{array}$ & $\begin{array}{c}\text { Right } \\
\text { lung }\end{array}$ \\
\hline \hline 1 & 0.0 & 0.0 & 0.0 & 0.0 & 0.0 & 0.0 & 0.0 & 0.0 \\
2 & 8.0 & 0.0 & 2.0 & 0.0 & 0.0 & 2.0 & 0.0 & 0.0 \\
3 & 2.0 & 5.0 & 8.0 & 0.0 & 0.0 & 6.0 & 0.0 & 0.0 \\
4 & 5.0 & 6.0 & 17.0 & 4.0 & 0.0 & 3.0 & 0.0 & 0.0 \\
5 & 1.0 & 0.0 & 0.0 & 0.0 & 0.0 & 1.0 & 0.0 & 0.0 \\
6 & 4.0 & 3.0 & 11.0 & 1.0 & 0.0 & 2.0 & 0.0 & 0.0 \\
7 & 1.0 & 0.0 & 1.0 & 0.0 & 0.0 & 0.0 & 0.0 & 0.0 \\
8 & 13.0 & 3.0 & 9.0 & 2.0 & 3.0 & 11.0 & 0.0 & 0.0 \\
\hline \hline
\end{tabular}

Table C.7.: 4Dopt $D_{\text {Mean }}$ for various OARs. Values are in Gy.

\begin{tabular}{c|c|c|c|c|c|c|c|c}
$\begin{array}{c}\text { Patient } \\
\text { Nr }\end{array}$ & Heart & $\begin{array}{c}\text { Spinal } \\
\text { cord }\end{array}$ & $\begin{array}{c}\text { Smaller } \\
\text { airways }\end{array}$ & Esophagus & Trachea & Aorta & $\begin{array}{c}\text { Left } \\
\text { lung }\end{array}$ & $\begin{array}{c}\text { Right } \\
\text { lung }\end{array}$ \\
\hline \hline 1 & 0.0 & 0.0 & 0.0 & 0.0 & 0.0 & 0.0 & 1.0 & 1.0 \\
2 & 1.0 & 0.0 & 1.0 & 0.0 & 0.0 & 0.0 & 3.0 & 3.0 \\
3 & 0.0 & 1.0 & 2.0 & 0.0 & 0.0 & 1.0 & 4.0 & 0.0 \\
4 & 1.0 & 1.0 & 7.0 & 2.0 & 0.0 & 1.0 & 2.0 & 2.0 \\
5 & 0.0 & 0.0 & 0.0 & 0.0 & 0.0 & 0.0 & 3.0 & 0.0 \\
6 & 0.0 & 0.0 & 3.0 & 0.0 & 0.0 & 1.0 & 4.0 & 2.0 \\
7 & 0.0 & 0.0 & 0.0 & 1.0 & 0.0 & 0.0 & 0.0 & 2.0 \\
8 & 1.0 & 0.0 & 3.0 & 1.0 & 1.0 & 1.0 & 8.0 & 5.0 \\
\hline \hline
\end{tabular}


Table C.8.: SBRT $D_{\text {Max }}$ for various OARs. Values are in Gy.

\begin{tabular}{c|c|c|c|c|c|c|c|c}
$\begin{array}{c}\text { Patient } \\
\mathrm{Nr}\end{array}$ & Heart & $\begin{array}{c}\text { Spinal } \\
\text { cord }\end{array}$ & $\begin{array}{c}\text { Smaller } \\
\text { airways }\end{array}$ & Esophagus & Trachea & Aorta & $\begin{array}{c}\text { Left } \\
\text { lung }\end{array}$ & $\begin{array}{c}\text { Right } \\
\text { lung }\end{array}$ \\
\hline \hline 1 & 11.0 & 10.0 & 23.0 & 11.0 & 0.0 & 19.0 & 26.0 & 26.0 \\
2 & 15.0 & 9.0 & 13.0 & 11.0 & 0.0 & 27.0 & 28.0 & 28.0 \\
3 & 13.0 & 9.0 & 14.0 & 14.0 & 4.0 & 25.0 & 27.0 & 12.0 \\
4 & 29.0 & 8.0 & 18.0 & 26.0 & 3.0 & 17.0 & 27.0 & 30.0 \\
5 & 23.0 & 3.0 & 0.0 & 6.0 & 0.0 & 6.0 & 26.0 & 4.0 \\
6 & 21.0 & 11.0 & 15.0 & 14.0 & 1.0 & 14.0 & 26.0 & 26.0 \\
7 & 8.0 & 9.0 & 18.0 & 7.0 & 0.0 & 8.0 & 10.0 & 27.0 \\
8 & 31.0 & 13.0 & 16.0 & 12.0 & 8.0 & 31.0 & 32.0 & 25.0 \\
\hline \hline
\end{tabular}

Table C.9.: SBRT $D_{\text {Threshold }}$ for various OARs. Values are in Gy.

\begin{tabular}{c|c|c|c|c|c|c|c|c}
$\begin{array}{c}\text { Patient } \\
\mathrm{Nr}\end{array}$ & Heart & $\begin{array}{c}\text { Spinal } \\
\text { cord }\end{array}$ & $\begin{array}{c}\text { Smaller } \\
\text { airways }\end{array}$ & Esophagus & Trachea & Aorta & $\begin{array}{c}\text { Left } \\
\text { lung }\end{array}$ & $\begin{array}{c}\text { Right } \\
\text { lung }\end{array}$ \\
\hline \hline 1 & 9.0 & 9.0 & 12.0 & 7.0 & 0.0 & 13.0 & 0.0 & 0.0 \\
2 & 10.0 & 9.0 & 7.0 & 6.0 & 0.0 & 11.0 & 0.0 & 0.0 \\
3 & 10.0 & 7.0 & 11.0 & 11.0 & 3.0 & 18.0 & 2.0 & 2.0 \\
4 & 12.0 & 7.0 & 15.0 & 6.0 & 1.0 & 7.0 & 0.0 & 0.0 \\
5 & 13.0 & 3.0 & 0.0 & 3.0 & 0.0 & 4.0 & 0.0 & 0.0 \\
6 & 16.0 & 10.0 & 12.0 & 12.0 & 0.0 & 11.0 & 0.0 & 0.0 \\
7 & 6.0 & 8.0 & 5.0 & 3.0 & 0.0 & 6.0 & 0.0 & 0.0 \\
8 & 22.0 & 11.0 & 11.0 & 8.0 & 5.0 & 23.0 & 0.0 & 6.0 \\
\hline \hline
\end{tabular}

Table C.10.: SBRT $D_{\text {Mean }}$ for various OARs. Values are in Gy.

\begin{tabular}{c|c|c|c|c|c|c|c|c}
$\begin{array}{c}\text { Patient } \\
\text { Nr }\end{array}$ & Heart & $\begin{array}{c}\text { Spinal } \\
\text { cord }\end{array}$ & $\begin{array}{c}\text { Smaller } \\
\text { airways }\end{array}$ & Esophagus & Trachea & Aorta & $\begin{array}{c}\text { Left } \\
\text { lung }\end{array}$ & $\begin{array}{c}\text { Right } \\
\text { lung }\end{array}$ \\
\hline \hline 1 & 4.0 & 2.0 & 3.0 & 2.0 & 0.0 & 2.0 & 3.0 & 3.0 \\
2 & 5.0 & 1.0 & 3.0 & 3.0 & 0.0 & 3.0 & 5.0 & 5.0 \\
3 & 6.0 & 2.0 & 6.0 & 5.0 & 1.0 & 6.0 & 9.0 & 2.0 \\
4 & 4.0 & 2.0 & 6.0 & 3.0 & 1.0 & 2.0 & 3.0 & 3.0 \\
5 & 4.0 & 1.0 & 0.0 & 1.0 & 0.0 & 1.0 & 6.0 & 1.0 \\
6 & 6.0 & 2.0 & 4.0 & 5.0 & 0.0 & 4.0 & 6.0 & 4.0 \\
7 & 2.0 & 2.0 & 3.0 & 4.0 & 0.0 & 2.0 & 1.0 & 3.0 \\
8 & 10.0 & 3.0 & 8.0 & 4.0 & 3.0 & 6.0 & 10.0 & 9.0 \\
\hline \hline
\end{tabular}




\section{C.3 Organs at risk margins}

Table C.11 shows the margins that were applied to OAR, before subtracting OAR plus margins from CTV in patients with complex geometry.

Table C.11.: OAR margins used for subtraction from CTV for ITV, 4Dopt and SBRT.

\begin{tabular}{|c|c|c|c|c|}
\hline \multirow{2}{*}{ Patient } & \multirow{2}{*}{ OAR } & \multicolumn{3}{|c|}{ Margin (mm) } \\
& & ITV & 4Dopt & SBRT \\
\hline \hline \multirow{2}{*}{ Patient 4 } & Smaller airways & 2 & 2 & 1 \\
& Esophagus & 0 & 0 & 1 \\
\hline Patient 5 & Heart & 0 & 0 & 2 \\
\hline \multirow{2}{*}{ Patient 6 } & Left lung Smaller airways & 3 & 2 & 11 \\
& Right lung smaller airways & 0 & 0 & 9 \\
\hline \multirow{2}{*}{ Patient 7 } & Esophagus & 2 & 2 & 0 \\
& Stomach & 0 & 0 & 1 \\
\hline \hline
\end{tabular}





\section{Bibliography}

[Ahlen, 1980] Ahlen, S. (1980). Theoretical and experimental aspects of the enery loss of relativistic heavily ionizing particles. Rev. Mod. Phys., 52(1):121.

[Alpen, 1998] Alpen, E. L. (1998). Radiation Biophysics. Academic Press, California, USA, 2nd edition edition.

[Amini et al., 2014] Amini, A., Yeh, N., Gaspar, L. E., Kavanagh, B., and Karam, S. D. (2014). Stereotactic body radiation therapy (sbrt) for lung cancer patients previously treated with conventional radiotherapy: a review. Radiation Oncology, 9(1):1-8.

[Barkas, 1963] Barkas, H. (1963). Nuclear Research Emulsions, volume 1. Academic Press, New York, USA and London, UK.

[Baumann et al., 2009] Baumann, P., Nyman, J., Hoyer, M., Wennberg, B., Gagliardi, G., Lax, I., Drugge, N., Ekberg, L., Friesland, S., Johansson, K. A., Lund, J. A., Morhed, E., Nilsson, K., Levin, N., Paludan, M., Sederholm, C., Traberg, A., Wittgren, L., and Lewensohn, R. (2009). Outcome in a prospective phase ii trial of medically inoperable stage i non-small-cell lung cancer patients treated with stereotactic body radiotherapy. Journal of Clinical Oncology, 27(20):3290-3296.

[Behringer et al., 2015] Behringer, P. A., Herz, C., Penzkofer, T., Tuncali, K., Tempany, C. M., and Fedorov, A. (2015). Open-source platform for prostate motion tracking during in-bore targeted mri-guided biopsy. 18(WS):122-9.

[Bender and Tomé, 2009] Bender, E. T. and Tomé, W. A. (2009). The utilization of consistency metrics for error analysis in deformable image registration. Physics in Medicine and Biology, 54(18):5561.

[Benedict et al., 2010] Benedict, S. H., Yenice, K. M., Followill, D., Galvin, J. M., Hinson, W., Kavanagh, B., Keall, P., Lovelock, M., Meeks, S., Papiez, L., Purdie, T., Sadagopan, R., Schell, M. C., Salter, B., Schlesinger, D. J., Shiu, A. S., Solberg, T., Song, D. Y., Stieber, V., Timmerman, R., Tome, W. A., Verellen, D., Wang, L., and Yin, F. F. (2010). Stereotactic body radiation therapy: the report of aapm task group 101. Medical Physics, 37(8):4078-4101.

[Benjamin et al., 1998] Benjamin, E. J., Wolf, P. A., D’Agostino, R. B., Silbershatz, H., Kannel, W. B., and Levy, D. (1998). Impact of atrial fibrillation on the risk of death: the framingham heart study. Circulation, 98(10):946-952. 
[Bert and Durante, 2011] Bert, C. and Durante, M. (2011). Motion in radiotherapy: particle therapy. Physics in Medicine and Biology, 56(16):R113-R144.

[Bert et al., 2012a] Bert, C., Engenhart-Cabillic, R., and Durante, M. (2012a). Particle therapy for noncancer diseases. Medical Physics, 39(4):1716-1727.

[Bert et al., 2009] Bert, C., Gemmel, A., Chaudhri, N., Lüchtenborg, R., Saito, N., Durante, M., and Rietzel, E. (2009). Rescanning to mitigate the impact of motion in scanned particle therapy. Report.

[Bert et al., 2008] Bert, C., Groezinger, S. O., and Rietzel, E. (2008). Quantification of interplay effects of scanned particle beams and moving targets. Phys. Med. Biol., 53(9):2253-2265.

[Bert et al., 2012b] Bert, C., Richter, D., Durante, M., and Rietzel, E. (2012b). Scanned carbon beam irradiation of moving films: comparison of measured and calculated response. Radiat. Oncol., 7(1):55.

[Bert and Rietzel, 2007] Bert, C. and Rietzel, E. (2007). 4D treatment planning for scanned ion beams. Radiat. Oncol., 2(24).

[Bethe, 1930] Bethe, H. (1930). Zur Theorie des Durchgangs schneller Korpuskularstrahlung durch Materie. Annalen der Physik, 5(5):325-400.

[Bloch, 1933] Bloch, F. (1933). Zur Bremsung rasch bewegter Teilchen beim Durchgang durch Materie. Annalen der Physik, 5(16):285-321.

[Bohr, 1940] Bohr, N. (1940). Scattering and stopping of fission fragments. Phys . Rev., 58(7):654- 655 .

[Boye et al., 2013] Boye, D., Lomax, T., and Knopf, A. (2013). Mapping motion from 4dmri to 3d-ct for use in 4d dose calculations: a technical feasibility study. Medical Physics, 40(6):061702.

[Bradley et al., 2015] Bradley, J. D., Paulus, R., Komaki, R., Masters, G., Blumenschein, G., Schild, S., Bogart, J., Hu, C., Forster, K., Magliocco, A., Kavadi, V., Garces, Y. I., Narayan, S., Iyengar, P., Robinson, C., Wynn, R. B., Koprowski, C., Meng, J., Beitler, J., Gaur, R., Curran, W. J., and Choy, H. (2015). Standard-dose versus high-dose conformal radiotherapy with concurrent and consolidation carboplatin plus paclitaxel with or without cetuximab for patients with stage iiia or iiib non-small-cell lung cancer (rtog 0617): a randomised, two-by-two factorial phase 3 study. The Lancet Oncology, 16(2):187-199.

[Breedveld et al., 2007] Breedveld, S., Storchi, P. R. M., Keijzer, M., Heemink, A. W., and Heijmen, B. J. M. (2007). A novel approach to multi-criteria inverse planning for imrt. Physics in Medicine and Biology, 52(20):6339. 
[Britton et al., 2007] Britton, K. R., Starkschall, G., Tucker, S. L., Pan, T., Nelson, C., Chang, J. Y., Cox, J. D., Mohan, R., and Komaki, R. (2007). Assessment of gross tumor volume regression and motion changes during radiotherapy for non-small-cell lung cancer as measured by fourdimensional computed tomography. Int. J. Radiat. Oncol. Biol. Phys., 68(4):1036-1046.

[Brock, 2010] Brock, K. K. (2010). Results of a multi-institution deformable registration accuracy study (midras). Int. J. Radiat. Oncol. Biol. Phys., 76(2):583-596.

[Brock et al., 2006] Brock, K. K., Dawson, L. A., Sharpe, M. B., Moseley, D. J., and Jaffray, D. A. (2006). Feasibility of a novel deformable image registration technique to facilitate classification, targeting and monitoring of tumor and normal tissue. Int. J. Radiat. Oncol. Biol. Phys., 64(4):1245-1254.

[Brown et al., 2007] Brown, W. T., Wu, X., Fayad, F., Fowler, J. F., Amendola, B. E., Garcia, S., Han, H., de la, Z. A., Bossart, E., Huang, Z., and Schwade, J. G. (2007). Cyberknife radiosurgery for stage I lung cancer: results at 36 months. Clin. Lung Cancer., 8(8):488-492.

[Cappato et al., 2005] Cappato, R., Calkins, H., Chen, S. A., Davies, W., Iesaka, Y., Kalman, J., Kim, Y. H., Klein, G., Packer, D., and Skanes, A. (2005). Worldwide survey on the methods, efficacy, and safety of catheter ablation for human atrial fibrillation. Circulation, 111(9):1100-1105.

[Cappato et al., 2010] Cappato, R., Calkins, H., Chen, S. A., Davies, W., Iseka, Y., Kalman, J., Kim, Y. H., Klein, G., Natale, A., Packer, D., Skanes, A., Ambrogi, F., and Biganzoli, E. (2010). Circulation.Arrhythmia and electrophysiology., volume 3, chapter Updated Worldwide Survey on the Methods, Efficacy, and Safety of Catheter Ablation for Human Atrial Fibrillation, pages 32-38.

[Carabe-Fernandez et al., 2007] Carabe-Fernandez, A., Dale, R. G., and Jones, B. (2007). The incorporation of the concept of minimum rbe (rbemin) into the linear-quadratic model and the potential for improved radiobiological analysis of high-let treatments. International Journal of Radiation Biology, 83(1):27-29.

[Castillo et al., 2009] Castillo, R., Castillo, E., Guerra, R., Johnson, V. E., McPhail, T., Garg, A. K., and Guerrero, T. (2009). A framework for evaluation of deformable image registration spatial accuracy using large landmark point sets. Physics in Medicine and Biology, 54(7):1849.

[Chatterjee and Schaefer, 1976] Chatterjee, A. and Schaefer, H. J. (1976). Microdosimetric structure of heavy ion tracks in tissue. Radiat. Environ. Biophys., 13:215-227.

[Chen et al., 2008] Chen, M., Lu, W., Chen, Q., Ruchala, K. J., and Olivera, G. H. (2008). A simple fixed-point approach to invert a deformation field. Med Phys, 35(1):81-88. 
[Chen et al., 2010] Chen, W., Craft, D., Madden, T. M., Zhang, K., Kooy, H. M., and Herman, G. T. (2010). A fast optimization algorithm for multicriteria intensity modulated proton therapy planning. Med Phys, 37(9):4938-4945.

[Chen et al., 2012] Chen, W., Unkelbach, J., Trofimov, A., Madden, T., Kooy, H., Bortfeld, T., and Craft, D. (2012). Including robustness in multi-criteria optimization for intensity-modulated proton therapy. Physics in Medicine and Biology, 57(3):591-608.

[Chheang and Brown, 2013] Chheang, S. and Brown, K. (2013). Lung cancer staging: Clinical and radiologic perspective. Semin Intervent Radiol, 30(2).

[Christensen and Johnson, 2001] Christensen, G. E. and Johnson, H. J. (2001). Consistent image registration. IEEE Transactions on Medical Imaging, 20(7):568-582.

[Christensen et al., 1996] Christensen, G. E., Rabbitt, R. D., and Miller, M. I. (1996). Deformable templates using large deformation kinematics. IEEE Transactions on Image Processing, 5(10):1435-1447.

[Chu et al., 1993] Chu, W. T., Ludewigt, B. A., and Renner, T. R. (1993). Instrumentation for treatment of cancer using proton and light-ion beams. Rev. Sci. Instrum., 64(8):2055-2122.

[Cleary and Peters, 2010] Cleary, K. and Peters, T. M. (2010). Image-guided interventions: Technology review and clinical applications. Annual Review of Biomedical Engineering, 12(1):119-142. PMID: 20415592.

[CRUK, 2016] CRUK (2016). Cancer research uk (original email from cruk) [cc by-sa 4.0 (http://creativecommons.org/licenses/by-sa/4.0)], via wikimedia commons.

[Dong et al., 2013a] Dong, P., Lee, P., Ruan, D., Long, T., Romeijn, E., Low, D. A., Kupelian, P., Abraham, J., Yang, Y., and Sheng, K. (2013a). 4pi noncoplanar stereotactic body radiation therapy for centrally located or larger lung tumors. International Journal of Radiation Oncology*Biology*Physics, 86(3):407 - 413.

[Dong et al., 2013b] Dong, P., Lee, P., Ruan, D., Long, T., Romeijn, E., Yang, Y., Low, D., Kupelian, P., and Sheng, K. (2013b). 4pi non-coplanar liver sbrt: A novel delivery technique. International Journal of Radiation Oncology*Biology*Physics, 85(5):1360 - 1366.

[Durante and Loeffler, 2010] Durante, M. and Loeffler, J. S. (2010). Charged particles in radiation oncology. Nat. Rev. Clin. Oncol., 7(1):37-43.

[Elsaesser et al., 2009] Elsaesser, T., Gemmel, A., Scholz, M., Schardt, D., and Krämer, M. (2009). Relevance of very low energy ions for heavy ion therapy. Phys. Med. Biol., 54(7):N101-N106. 
[Elsaesser and Scholz, 2006] Elsaesser, T. and Scholz, M. (2006). Improvement of the local effect model (LEM)-implications of clustered DNA damage. Radiat. Prot. Dosimetry., 122(14):475-477.

[Elsaesser and Scholz, 2007] Elsaesser, T. and Scholz, M. (2007). Cluster effects within the local effect model. Radiat. Res., 167(3):319-329.

[Elsaesser et al., 2010] Elsaesser, T., Weyrather, W. K., Friedrich, T., Durante, M., Iancu, G., Krämer, M., Kragl, G., Brons, S., Winter, M., Weber, K. J., and Scholz, M. (2010). Quantification of the relative biological effectiveness for ion beam radiotherapy: direct experimental comparison of proton and carbon ion beams and a novel approach for treatment planning. Int. J. Radiat. Oncol. Biol. Phys., 78(4):1177-1183.

[Engelsman and Bert, 2011] Engelsman, M. and Bert, C. (2011). Precision and uncertainties in proton therapy for moving targets. In Francis, T. ., editor, Proton Therapy Physics. CRC Press, Boca Raton, USA.

[Fakiris et al., 2009] Fakiris, A. J., McGarry, R. C., Yiannoutsos, C. T., Papiez, L., Willams, M., Henderson, M. A., and Timmerman, R. (2009). Stereotactic body radiation therapy for earlystage non-small-cell lung carcinoma: four-year results of a prospective phase ii study. International Journal of Radiation Oncology, 75(3):677-682.

[Fassi et al., 2014] Fassi, A., Schaerer, J., Fernandes, M., Riboldi, M., Sarrut, D., and Baroni, G. (2014). Tumor tracking method based on a deformable $4 d$ \{CT $\}$ breathing motion model driven by an external surface surrogate. International Journal of Radiation Oncology *Bology*Physics, 88(1):182 - 188.

[Fassi et al., 2015] Fassi, A., Seregni, M., Riboldi, M., Cerveri, P., Sarrut, D., Ivaldi, G. B., de Fatis, P. T., Liotta, M., and Baroni, G. (2015). Surrogate-driven deformable motion model for organ motion tracking in particle radiation therapy. Physics in Medicine and Biology, $60(4): 1565$.

[Fedorov et al., 2012] Fedorov, A., Beichel, R., Kalpathy-Cramer, J., Finet, J., Fillion-Robin, J.C., Pujol, S., Bauer, C., Jennings, D., Fennessy, F. M., Sonka, M., Buatti, J., Aylward, S. R., Miller, J. V., Pieper, S., and Kikinis, R. (2012). 3d slicer as an image computing platform for the quantitative imaging network. Magn Reson Imaging, 30(9):1323 - 1341.

[Fedorov et al., 2015] Fedorov, A., Khallaghi, S., Sánchez, C., Lasso, A., Fels, S., Tuncali, K., Sugar, E. N., Kapur, T., Zhang, C., Wells, W., Nguyen, P., Abolmaesumi, P., and Tempany, C. (2015). Open-source image registration for mri-trus fusion-guided prostate interventions. Int $J$ Comput Assist Radiol Surg, 10(6):925-34. 
[Flampouri et al., 2006] Flampouri, S., Jiang, S., Sharp, G. C., Wolfgang, J., Patel, A. A., and Choi, N. C. (2006). Estimation of the delivered patient dose in lung imrt treatment based on deformable registration of 4d-ct data and monte carlo simulations. Phys Med Biol, 51(11):2763-2779.

[Fowler, 1989] Fowler, J. F. (1989). The Linear-Quadratic Formula and Progress in Fractionated Radiotherapy. Brit. J. Radiol., 62(740):679-694.

[Fredriksson et al., 2011] Fredriksson, A., Forsgren, A., and Hårdemark, B. (2011). Minimax optimization for handling range and setup uncertainties in proton therapy. Medical Physics, 38(3):1672-1684.

[Friedrich et al., 2014] Friedrich, T., Sholz, U., Durante, M., and Sholz, M. (2014). Rbe of ion beams in hypofractionated radiotherapy (sbrt). Phys.Med., 30(5):588-591.

[Fuks and Kolesnick, 2005] Fuks, Z. and Kolesnick, R. (2005). Engaging the vascular component of the tumor response. Cancer Cell, 8(2):89-91.

[Furukawa et al., 2007] Furukawa, T., Inaniwa, T., Sato, S., Tomitani, T., Minohara, S., Noda, K., and Kanai, T. (2007). Design study of a raster scanning system for moving target irradiation in heavy-ion radiotherapy. Med. Phys., 34(3):1085-1097.

[Garcia-Barros et al., 2003] Garcia-Barros, M., Paris, F., Cordon-Cardo, C., Lyden, D., Rafii, S., Haimovitz-Friedman, A., Fuks, Z., and Kolesnick, R. (2003). Tumor response to radiotherapy regulated by endothelial cell apoptosis. Science, 300(5622):1155-1159.

[Geiss et al., 1999] Geiss, O. B., Schardt, D., Voss, B., Krämer, M., and Kraft, G. (1999). Correlation between CT number and water equivalent thickness. Report.

[Gemmel et al., 2011] Gemmel, A., Rietzel, E., Kraft, G., Durante, M., and Bert, C. (2011). Calculation and experimental verification of the RBE-weighted dose for scanned ion beams in the presence of target motion. Phys. Med. Biol., 56:7337-7351.

[Georg et al., 2008] Georg, D., Hillbrand, M., Stock, M., Dieckmann, K., and Pötter, R. (2008). Can protons improve sbrt for lung lesions?: Dosimetric considerations. Radiotherapy and Oncology, 88(3):368-375.

[German Federal Statistical Office, 2015] German Federal Statistical Office (2015). Krebs war 2013 die zeithäufigste todesursache. Official Destatis press release No. 034.

[Graeff, 2014] Graeff, C. (2014). Motion mitigation in scanned ion beam therapy through 4doptimization. Phys.Med., 30(5):570-577. 
[Graeff et al., 2012] Graeff, C., Durante, M., and Bert, C. (2012). Motion mitigation in intensity modulated particle therapy by internal target volumes covering range changes. Medical Physics, 39(10):6004-6013.

[Graeff et al., 2015] Graeff, C., Lehmann, H. I., Constantinescu, A., Simoniello, P., Lugenbiel, P., Prall, M., Richter, D., Takami, M., Eichhorn, A., Erbeldinger, N., Fournier, C., Kaderka, R., Helmbrecht, S., Fiedler, F., Debus, J., Thomas, D., Bert, C., Durante, M., and Packer, D. L. (2015). Catheter-free arrhythmia ablation using scanned carbon ion beams in a porcine model. In GSI Report 2015.

[Graeff et al., 2013] Graeff, C., Lüchtenborg, R., Eley, J. G., Durante, M., and Bert, C. (2013). A 4d-optimization concept for scanned ion beam therapy. Radiotherapy and Oncology, 109(3):419 - 424.

[Greco et al., 2011] Greco, C., Zelefsky, M. J., Lovelock, M., Fuks, Z., Hunt, M., Rosenzweig, K., Zatcky, J., Kim, B., and Yamada, Y. (2011). Predictors of local control after singledose stereotactic image-guided intensity-modulated radiotherapy for extracranial metastases. Int.J.Radiat.Oncol.Biol.Phys., 79(4):1151-1157.

[Groezinger, 2004] Groezinger, S. O. (2004). Volume Conformal Irradiation of Moving Target Volumes with Scanned Ion Beams. Thesis/dissertation, TU Darmstadt.

[Grutters et al., 2010] Grutters, J. P. C., Kessels, A. G. H., Pijls-Johannesma, M., Ruysscher, D., oore, M. A. ., and Lambin, P. (2010). Comparison of the effectiveness of radiotherapy with photons, protons and carbon-ions for non-small cell lung cancer: A meta-analysis. Radiotherapy and Oncology, 95(1):32-40.

[Haberer et al., 1993] Haberer, T., Becher, W., Schardt, D., and Kraft, G. (1993). Magnetic scanning system for heavy ion therapy. Nucl. Instrum. Meth. A, 330:296-305.

[Habermehl et al., 2013] Habermehl, D., Debus, J., Ganten, T., Ganten, M.-K., Bauer, J., Brecht, I. C., Brons, S., Haberer, T., Haertig, M., Jäkel, O., Parodi, K., Welzel, T., and Combs, S. E. (2013). Hypofractionated carbon ion therapy delivered with scanned ion beams for patients with hepatocellular carcinoma - feasibility and clinical response. Radiation Oncology, 8(1):18.

[Halasz and Rockhill, 2013] Halasz, L. M. and Rockhill, J. K. (2013). Stereotactic radiosurgery and stereotactic radiotherapy for brain metastases. Surg Neurol Int., 4(4):S185-S191.

[Hardcastle et al., 2012] Hardcastle, N., Tomé, W. A., Cannon, D. M., Brouwer, C. L., Wittendorp, P. W., Dogan, N., Guckenberger, M., Allaire, S., Mallya, Y., Kumar, P., Oechsner, M., 
Richter, A., Song, S., Myers, M., Polat, B., and Bzdusek, K. (2012). A multi-institution evaluation of deformable image registration algorithms for automatic organ delineation in adaptive head and neck radiotherapy. Radiat Oncol, 7:90-90.

[Hartman et al., 2015] Hartman, J., Kontaxis, C., Bol, G. H., Frank, S. J., Lagendijk, J. J. W., van Vulpen, M., and Raaymakers, B. W. (2015). Dosimetric feasibility of intensity modulated proton therapy in a transverse magnetic field of $1.5 \mathrm{t}$. Physics in Medicine and Biology, 60(15):5955.

[Heath and Seuntjens, 2006] Heath, E. and Seuntjens, J. (2006). A direct voxel tracking method for four-dimensional monte carlo dose calculations in deforming anatomy. Medical Physics, 33(2).

[Hecht, 2012] Hecht, S. S. (2012). Lung carcinogenesis by tobacco smoke. International Journal of Cancer, 131(12).

[Herrell et al., 2012] Herrell, S. D., Galloway, R. L., and Su, L. M. (2012). Image-guided robotic surgery: update on research and potential applications in urologic surger. Curr Opin Urol, 22(1):47-54.

[Hill et al., 2001] Hill, D. L., Batchelor, P. G., Holden, M., and Hawkes, D. J. (2001). Medical image registration. Phys. Med. Biol., 46(3):R1-45.

[Huttenlocher et al., 1993] Huttenlocher, D. P., Klanderman, G. A., and Rucklidge, W. J. (1993). Comparing images using the hausdorff distance. IEEE Transactions on Pattern Analysis and Machine Intelligence, 15(9):850-863.

[ICRU, 1993a] ICRU (1993a). Prescribing, recording and reporting photon beam therapy. ICRU report no. 50 .

[ICRU, 1993b] ICRU (1993b). Report 50. Report.

[ICRU, 1999] ICRU (1999). Prescribing, recording and reporting photon beam therapy, supplement to ICRU report 50. ICRU report no. 62.

[ICRU, 2007] ICRU (2007). ICRU report 78: Prescribing, recording and reporting proton-beam therapy. Journal of the ICRU, 7(2).

[Iyengar et al., 2014] Iyengar, P., Kavanagh, B. D., Wardak, Z., Smith, I., Ahn, C., Gerber, D. E., Dowell, J., Hughes, R., Camidge, D. R., Gaspar, L. E., Doebele, R. C., Bunn, P. A., Choy, H., and Timmerman, R. (2014). Phase ii trial of stereotactic body radiation therapy combined with erlotinib for patients with limited but progressive metastatic non-small-cell lung cancer. J.Clin.Oncol., 32(34):3824-3854. 
[J. and M., 2010] J., A. A. and M., S. J. (2010). Murray \& Nadel's Textbook of Respiratory Medicine (5th ed.). Saunders Elsevier, Philadelphia, USA.

[Jäkel et al., 2001] Jäkel, O., Jacob, C., Schardt, D., Karger, C. P., and Hartmann, G. H. (2001). Relation between carbon ion ranges and x-ray ct numbers. Med Phys, 28:701-703.

[January et al., 2014] January, C. T., Wann, L. S., Alpert, J. S., Calkins, H., Cigarroa, J. E., Cleveland, Jr., J. C., Conti, J. B., Ellinor, P. T., Ezekowitz, M. D., Field, M. E., Murray, K. T., Sacco, R. L., Stevenson, W. G., Tchou, P. J., Tracy, C. M., and Yancy, C. W. (2014). 2014 aha/acc/hrs guideline for the management of patients with atrial fibrillationa report of the american college of cardiology/american heart association task force on practice guidelines and the heart rhythm society. Journal of the American College of Cardiology, 64(21):e1-e76.

[K. et al., 2005] K., S.-A., A., P., K., N., P., S., and M., T. (2005). Survival of patients with advanced non-small-cell lung cancer at ubon ratchathani cancer center, thailand. Southeast Asian J Trop Med Public Health, (4):994-1006.

[Kaatsch et al., 2014] Kaatsch, P., Spix, C., Hentschel, S., Katalinic, A., Luttmann, S., Stegmaier, C., Caspritz, S., Cernaj, J., Ernst, A., Folkerts, J., Hansmann, J., Kranzhofer, K., KrieghoffHenning, E., Kunz, B., Penzkofer, A., Treml, K., Wittenberg, K., Baras, N., Barnes, B., Bertz, J., Buttmann-Schweiger, N., Dahm, S., Franke, M., Haberland, J., Kraywinkel, K., Wienecke, A., and Wolf, U. (2014). Cancer in germany 2009/2012. Report.

[Kadoya et al., 2010] Kadoya, N., Obata, Y., Kato, T., Kagiya, M., Nakamura, T., Tomoda, T., Takada, A., Takayama, K., and Fuwa, N. (2010). Dose-volume comparison of proton radiotherapy and stereotactic body radiotherapy for non-small-cell lung cancer. Int.J.Radiat.Oncol.Biol.Phys., 79(4):1225-1231.

[Kamada et al., 2016] Kamada, T., Tsujii, H., Blakely, E. A., Debus, J., De Neve, W., Durante, M., Jäkel, O., Mayer, R., Orecchia, R., Pötter, R., Vatnitsky, S., and Chu, W. T. (2016). Carbon ion radiotherapy in japan: an assessment of 20 years of clinical experience. The Lancet Oncology, 16(2):e93-e100.

[Kashani et al., 2008] Kashani, R., Hub, M., Balter, J. M., Kessler, M. L., Dong, L., Zhang, L., Xing, L., Xie, Y., Hawkes, D., Schnabel, J. A., McClelland, J., Joshi, S., Chen, Q., and Lu, W. (2008). Objective assessment of deformable image registration in radiotherapy: A multiinstitution study. Medical Physics, 35(12):5944-5953.

[Kashani et al., 2007] Kashani, R., Hub, M., Kessler, M. L., and Balter, J. M. (2007). Technical note: A physical phantom for assessment of accuracy of deformable alignment algorithms. Medical Physics, 34(7):2785-2788. 
[Kasper et al., 2015] Kasper, D. L., Hauser, S. L., Jameson, J. L., Fauci, A. S., Longo, D. L., and Loscalzo, J. (2015). Harrison's Principles of Internal Medicine (19th ed.). McGraw-Hill, Columbus, USA.

[Katz and Cucinotta, 1999] Katz, R. and Cucinotta, F. (1999). Tracks to therapy. Radiat. Meas., 31(1-6):379-388.

[Keall et al., 2014] Keall, P. J., Colvill, E., O?Brien, R., Ng, J. A., Poulsen, P. R., Eade, T., Kneebone, A., and Booth, J. T. (2014). The first clinical implementation of electromagnetic transponder-guided mlc tracking. Medical Physics, 41(2).

[Keall et al., 2001] Keall, P. J., Kini, V. R., Vedam, S. S., and Mohan, R. (2001). Motion adaptive X-ray therapy: a feasibility study. Phys. Med. Biol., 46(1):1-10.

[Kiefer and Straaten, 1986] Kiefer, J. and Straaten, H. (1986). A model of ion track structure based on classical collision dynamics. Phys. Med. Biol., 31(11):1201-1209.

[Kilby et al., 2010] Kilby, W., Dooley, J. R., Kuduvalli, G., Sayeh, S., and C. R. Maurer, J. (2010). The cyberknife robotic radiosurgery system in 2010. Technol. Cancer Res. Treat., 9(5):433452.

[Kirby et al., 2011] Kirby, N., Chuang, C., and Pouliot, J. (2011). A two-dimensional deformable phantom for quantitatively verifying deformation algorithms. Medical Physics, $38(8): 4583-4586$.

[Klein et al., 2009] Klein, A., Andersson, J., Ardekani, B. A., Ashburner, J., Avants, B., Chiang, M.-C., Christensen, G. E., Collins, D. L., Gee, J., Hellier, P., Song, J. H., Jenkinson, M., Lepage, C., Rueckert, D., Thompson, P., Vercauteren, T., Woods, R. P., Mann, J. J., and Parsey, R. V. (2009). Evaluation of 14 nonlinear deformation algorithms applied to human brain mri registration. Neuroimage, 46(3):786-802.

[Kraft, 2000] Kraft, G. (2000). Tumor therapy with heavy charged particles. Progress in Particle and Nuclear Physics, 45(Supplement 2):S473-S544.

[Krämer, 1995] Krämer, M. (1995). Calculation of heavy-ion track structure. Nucl. Instrum. Meth. B, 105(1-4):14-20.

[Krämer, 2009] Krämer, M. (2009). Swift ions in radiotherapy - treatment planning with TRiP98. Nucl. Instrum. Meth. B, 267(6):989-992.

[Krämer and Durante, 2010] Krämer, M. and Durante, M. (2010). Ion beam transport calculations and treatment plans in particle therapy. Eur. Phys. J. D, 60:8 pages. 
[Krämer et al., 2000] Krämer, M., Jäkel, O., Haberer, T., Kraft, G., Schardt, D., and Weber, U. (2000). Treatment planning for heavy-ion radiotherapy: physical beam model and dose optimization. Phys. Med. Biol., 45(11):3299-3317.

[Krämer and Scholz, 2000] Krämer, M. and Scholz, M. (2000). Treatment planning for heavyion radiotherapy: calculation and optimization of biologically effective dose. Phys. Med. Biol., 45(11):3319-3330.

[Krämer et al., 2003] Krämer, M., Wang, J. F., and Weyrather, W. (2003). Biological dosimetry of complex ion radiation fields. Phys. Med. Biol., 48(14):2063-2070.

[Lagendijk et al., 2016] Lagendijk, J. J. W., van Vulpen, M., and Raaymakers, B. W. (2016). The development of the mri linac system for online mri-guided radiotherapy: a clinical update. Journal of Internal Medicine.

[Langen and Jones, 2001] Langen, K. M. and Jones, D. T. L. (2001). Organ motion and its management. Int. J. Radiat. Oncol. Biol. Phys., 50(1):265-278.

[Lehmann et al., 2015] Lehmann, H. I., Richter, D., Prokesch, H., Graeff, C., Prall, M., Simoniello, P., Fournier, C., Bauer, J., Kaderka, R., Weymann, A., Szabó, G., Sonnenberg, K., Constantinescu, A. M., Johnson, S. B., Misiri, J., Takami, M., Miller, R. C., Herman, M. G., Asirvatham, S. J., Brons, S., Jäkel, O., Haberer, T., Debus, J., Durante, M., Bert, C., and Packer, D. L. (2015). Atrioventricular node ablation in langendorff-perfused porcine hearts using carbon ion particle therapy: Methods and an in vivo feasibility investigation for catheter-free ablation of cardiac arrhythmias. Circulation: Arrhythmia and Electrophysiology, 8(2):429-438.

[Leow et al., 2007] Leow, A. D., Yanovsky, I., Chiang, M. C., Lee, A. D., Klunder, A. D., Lu, A., Becker, J. T., Davis, S. W., Toga, A. W., and Thompson, P. M. (2007). Statistical properties of jacobian maps and the ralization of unbiased large-deformation nonlinear image registration. IEEE Trans Med, 26:822-832.

[Li et al., 2015a] Li, M., Miller, K., Joldes, G., Doyle, B., Garlapati, R., Kikinis, R., and Wittek, A. (2015a). Patient-specific biomechanical model as whole-body ct image registration tool. Med Image Anal, 22(1):22-34.

[Li et al., 2015b] Li, M., Wittek, A., Joldes, G., and Miller, K. (2015b). Fuzzy tissue classification for non-linear patient-specific biomechanical models for whole-body image registration.

[Lilley, 2006] Lilley, J. (2006). Nuclear Physics - Principles and Applications. Wiley, Manchester, UK. 
[Liu et al., 2012] Liu, F., Hu, Y., Zhang, Q., Kincaid, R., Goodman, K. A., and Mageras, G. S. (2012). Evaluation of deformable image registration and motion model in ct images with limited features. Phys Med Biol, 57(9):2539-2554. 22491010[pmid].

[Liu et al., 2007] Liu, H. H., Balter, P., Tutt, T., Choi, B., Zhang, J., Wang, C., Chi, M., Luo, D., Pan, T., Hunjan, S., Starkschall, G., Rosen, I., Prado, K., Liao, Z., Chang, J., Komaki, R., Cox, J. D., Mohan, R., and Dong, L. (2007). Assessing respiration-induced tumor motion and internal target volume using four-dimensional computed tomography for radiotherapy of lung cancer. Int. J. Radiat. Oncol. Biol. Phys., 68(2):531-540.

[Liu et al., 2016a] Liu, S., Cai, W., Pujol, S., Kikinis, R., and Feng, D. (2016a). Cross-view neuroimage pattern analysis in alzheimer's disease staging. Front Aging Neurosci, 8:23-.

[Liu et al., 2015] Liu, S., Liu, S., Cai, W., Che, H., Pujol, S., Kikinis, R., Feng, D., Fulham, M., and ADNI (2015). Multimodal neuroimaging feature learning for multiclass diagnosis of alzheimer's disease. IEEE Trans Biomed Eng, 62(4):1132-40.

[Liu et al., 2016b] Liu, W., Schild, S. E., Chang, J. Y., Liao, Z., Chang, Y.-H., Wen, Z., Shen, J., Stoker, J. B., Ding, X., Hu, Y., Sahoo, N., Herman, M. G., Vargas, C., Keole, S., Wong, W., and Bues, M. (2016b). Exploratory study of 4d versus 3d robust optimization in intensity modulated proton therapy for lung cancer. International Journal of Radiation Oncology, 95(1):523-533.

[Lo et al., 2010] Lo, S. S., Fakiris, A. J., Chang, E. L., Mayr, N. A., Wang, J. Z., Papiez, L., Teh, B. S., McGarry, R. C., Cardenes, H. R., and Timmerman, R. D. (2010). Stereotactic body radiation therapy: a novel treatment modality. Nat Rev Clin Oncol, 7(1):44-54.

[Lu et al., 2006a] Lu, H. M., Safai, S., Schneider, R., Adams, J. A., Chen, Y. L., Wolfgang, J. A., Sharp, G. C., Brett, R. C., Kirch, D. G., Hong, T. S., Delaney, T. F., Jiang, S. B., Kooy, H. M., and Chen, G. T. Y. (2006a). Respiratory-gated proton therapy treatment. In Abstract Book PTCOG 44.

[Lu et al., 2006b] Lu, W., Olivera, G. H., Chen, Q., Chen, M. L., and Ruchala, K. J. (2006b). Automatic re-contouring in 4d radiotherapy. Phys Med Biol, 51(5):1077-1099.

[Lu et al., 2006c] Lu, W., Olivera, G. H., Chen, Q., Chen, M. L., and Ruchala, K. J. (2006c). Automatic re-contouring in 4D radiotherapy. Phys. Med. Biol., 51(5):1077-1099.

[Mak and Truong, 2012] Mak, G. S. and Truong, Q. A. (2012). Cardiac ct: Imaging of and through cardiac devices. Current cardiovascular imaging reports, 5(5):328-336.

[Malinowski et al., 2011] Malinowski, K., McAvoy, T. J., George, R., Dietrich, S., and D'Souza, W. D. (2011). Incidence of changes in respiration-induced tumor motion and its relationship 
with respiratory surrogates during individual treatment fractions. International Journal of Radiation Oncology, 82(5):1665-1673.

[McInerney and Terzopoulos, 1996] McInerney, T. and Terzopoulos, D. (1996). Deformable models in medical image analysis: a survey. Med. Image Anal., 1(2):91-108.

[Meyer et al., 2015] Meyer, A., Lasso, A., Ungi, T., and Fichtinger, G. (2015). Live ultrasound volume reconstruction using scout scanning. 9415:94152A-94152A-9.

[Minohara et al., 2000] Minohara, S., Kanai, T., Endo, M., Noda, K., and Kanazawa, M. (2000). Respiratory gated irradiation system for heavy-ion radiotherapy. Int. J. Radiat. Oncol. Biol. Phys., 47(4):1097-1103.

[Mitaroff et al., 1998] Mitaroff, A., Kraft-Weyrather, W., Geiss, O. B., and Kraft, G. (1998). Biological verification of heavy ion treatment planning. Radiat. Environ. Biophys., 37(1):47-51.

[Molière, 1948] Molière, G. (1948). Theorie der streuung schneller geladener teilchen ii, mehrfach- und vielfachstreuung. Zeitschrift für Naturforschung, 3a:78-97.

[Moore et al., 2004] Moore, C. S., Liney, G. P., and Beavis, A. W. (2004). Quality assurance of registration of ct and mri data sets for treatment planning of radiotherapy for head and neck cancers. J Appl Clin Med Phys, 5(1):25-35.

[Mori et al., 2013] Mori, S., Furukawa, T., Inaniwa, T., Zenklusen, S., Nakao, M., Shirai, T., and Noda, K. (2013). Systematic evaluation of four-dimensional hybrid depth scanning for carbon-ion lung therapy. Med Phys., 40(3):031720.

[Mori et al., 2016] Mori, S., Karube, M., Shirai, T., Tajiri, M., Takekoshi, T., Miki, K., Shiraishi, Y., Tanimoto, K., Shibayama, K., Yasuda, S., Yamamoto, N., Yamada, S., Tsuji, H., Noda, K., and Kamada, T. (2016). Carbon-ion pencil beam scanning treatment with gated markerless tumor tracking: An analysis of positional accuracy. International Journal of Radiation Oncology, 95(1):258-266.

[Mori et al., 2009] Mori, S., Lu, H., Wolfgang, J. A., Choi, N. C., and Chen, G. T. Y. (2009). Effects of interfractional anatomical changes on Water-Equivalent pathlength in ChargedParticle radiotherapy of lung cancer. J. Radiat. Res., 50(6):513-519.

[Munro, 1970] Munro, T. R. (1970). The relative radiosensitivity of the nucleus and cytoplasm of chinese hamster fibroblasts. Radiat. Res., 42(3):451-470.

[Mutic et al., 2001] Mutic, S., Dempsey, J. F., Bosch, W. R., Low, D. A., Drzymala, R. E., Chao, K. S., Goddu, S. M., Cutler, P. D., and Purdy, J. A. (2001). Multimodality image registration quality assurance for conformal three-dimensional treatment planning. Int $J$ Radiat Oncol Biol Phys, 51(1):255-260. 
[Naini et al., 2010] Naini, A. S., Patel, R. V., and Samani, A. (2010). Ct-enhanced ultrasound image of a totally deflated lung for image-guided minimally invasive tumor ablative procedures. IEEE Transactions on Biomedical Engineering, 57(10):2627-2630.

[NCI, 1988] NCI (1988). https://visualsonline.cancer.gov/details.cfm?imageid=2348.

[Newhauser and Durante, 2011] Newhauser, W. D. and Durante, M. (2011). Assessing the risk of second malignancies after modern radiotherapy. Nat.Rev.Cancer, 11:434-448.

[Nithiananthan et al., 2011] Nithiananthan, S., Schafer, S., Uneri, A., Mirota, D. J., Stayman, J. W., Zbijewski, W., Brock, K. K., Daly, M. J., Chan, H., Irish, J. C., and Siewerdsen, J. H. (2011). Demons deformable registration of ct and cone-beam ct using an iterative intensity matching approach. Medical Physics, 38(4):1785-1798.

[Ong et al., 2016] Ong, C., Verbakel, W. F. A. R., Cuijpers, J. P., Slotman, B. J., and Senan, S. (2016). Dosimetric impact of interplay effect on rapidarc lung stereotactic treatment delivery. International Journal of Radiation Oncology, 79(1):305-311.

[Paganetti et al., 2005] Paganetti, H., Jiang, H., and Trofimov, A. (2005). 4D monte carlo simulation of proton beam scanning: modelling of variations in time and space to study the interplay between scanning pattern and time-dependent patient geometry. Phys. Med. Biol., 50(5):983-990.

[Paganetti et al., 2002] Paganetti, H., Niemierko, A., Ancukiewicz, M., Gerweck, L. E., Goitein, M., Loeffler, J. S., and Suit, H. D. (2002). Relative biological effectiveness (RBE) values for proton beam therapy. Int. J. Radiat. Oncol. Biol. Phys., 53(2):407-421.

[Paretzke, 1986] Paretzke, H. G. (1986). Physical events of heavy ion interactions with matter. Adv. Space Res., 6(11):67-73.

[Park et al., 2012a] Park, H. J., Griffin, R. J., Hui, S., Levitt, S. H., and Song, C. W. (2012a). Radiation-induced vascular damage in tumors: implications of vascular damage in ablative hypofractionated radiotherapy (sbrt and srs). Radiat Res, 177(3):311-327.

[Park et al., 2012b] Park, P. C., Zhu, X. R., Lee, A. K., Sahoo, N., Melancon, A. D., Zhang, L., and Dong, L. (2012b). A beam-specific planning target volume (ptv) design for proton therapy to account for setup and range uncertainties. Int.J.Radiat.Oncol.Biol.Phys., 82(2):e329-e336.

[Perrin et al., 2014] Perrin, R., Peroni, M., Bernatowicz, K., Zakova, M., Knopf, A., Safai, S., and Parkel, T. (2014). A realistic breathing phantom of the thorax for testing new motion mitigation techniques with scanning proton therapy. Med Phys, 41(6):111.

[Perrin et al., 2016] Perrin, R. L., Peroni, M., Bernatowicz, K., Schaetti, A., Knopf, A. K., Zakova, M., Oxley, D., Mayor, A., Safai, S., Parkel, T. C., Weber, D. C., and Lomax, T. (2016). 
Oc-0484: Rescanning measurements in a 4d anthropomorphic phantom for evaluation of motion-mitigated, pbs proton therapy. Radiotherapy and Oncology, 115:S238-S239.

[Pflugfelder et al., 2008] Pflugfelder, D., Wilkens, J. J., and Oelfke, U. (2008). Worst case optimization: a method to account for uncertainties in the optimization of intensity modulated proton therapy. Phys Med Biol, 53(6):1689-1700.

[Phillips et al., 1992] Phillips, M. H., Pedroni, E., Blattmann, H., Boehringer, T., Coray, A., and Scheib, S. (1992). Effects of respiratory motion on dose uniformity with a charged particle scanning method. Phys. Med. Biol., 37(1):223-233.

[Pinter et al., 2012] Pinter, C., Lasso, A., Wang, A., Jaffray, D., and Fichtinger, G. (2012). Slicerrt - radiation therapy research toolkit for 3d slicer. Med. Phys., 39(10):6332-6338.

[Plastimatch, 2016] Plastimatch (2016). http://plastimatch.org.

[Polacin et al., 1992] Polacin, A., Kalender, W. A., and Marchal, G. (1992). Evaluation of section sensitivity profiles and image noise in spiral ct. Radiology, 185(1):29-35.

[Prall et al., 2016a] Prall, M., Durante, M., nad B. Przybyla, T. B., Graeff, C., nad C. LaTessa, P. M. L., Shestov1, L., Simoniello1, P., Danly, C., Mariam, F., Merrill, F., Nedrow, P., Wilde, C., and Varentsov, D. (2016a). High-energy proton imaging for biomedical applications. Scientific Reports, Accepted.

[Prall et al., 2016b] Prall, M., Hild, S., Anderle, K., and Graeff, C. (2016b). Treatment planning with an adaptive dose grid. In Abstract Book PTCOG 55.

[Pujol et al., 2016] Pujol, S., Baldwin, M., Nassiri, J., Kikinis, R., and Shaffer, K. (2016). Using 3d modeling techniques to enhance teaching of difficult anatomical concepts. Acad Radiol, 23(4):507-16.

[Ramalingam and Belani, 2008] Ramalingam, S. and Belani, C. (2008). Systemic chemotherapy for advanced non-small cell lung cancer: Recent advances and future directions. The Oncologist, 13(suppl 1):5-13.

[Rami-Porta et al., 2009] Rami-Porta, R., Crowley, J. J., and Goldstraw, P. (2009). The revised tnm staging system for lung cancer. Ann Thorac Cardiovasc Surg, 15(1).

[Register et al., 2010] Register, S. P., Zhang, X., Mohan, R., and Chang, J. Y. (2010). Proton stereotactic body radiation therapy for clinically challenging cases of centrally and superiorly located stage i non-small-cell lung cancer. Int.J.Radiat.Oncol.Biol.Phys., 80(4):1015-1022.

[Rey et al., 2002] Rey, D., Subsol, G., Delingette, H., and Ayache, N. (2002). Automatic detection and segmentation of evolving processes in $3 \mathrm{~d}$ medical images: application to multiple sclerosis. Med Image Anal, 6(2):163-179. 
[Ricardi et al., 2010] Ricardi, U., Filippi, A. R., Guarneri, A., Giglioli, F. R., Ciammella, P., Franco, P., Mantovani, C., Borasio, P., Scagliotti, G. V., and Ragona, R. (2010). Stereotactic body radiation therapy for early stage non-small cell lung cancer: Results of a prospective trial. Lung Cancer, 68(1):72-77.

[Richter, 2012] Richter, D. (2012). Treatment planning for tumors with residual motion in scanned ion beam therapys. Thesis/dissertation, TU Darmstadt.

[Richter et al., 2014] Richter, D., Graeff, C., Jakel, O., Combs, S. E., Durante, M., and Bert, C. (2014). Residual motion mitigation in scanned carbon ion beam therapy of liver tumors using enlarged pencil beam overlap. Radiotherapy and Oncology, 113(2):290-295.

[Richter et al., 2013] Richter, D., Schwarzkopf, A., Trautmann, J., Kramer, M., Durante, M., Jakel, O., and Bert, C. (2013). Upgrade and benchmarking of a 4d treatment planning system for scanned ion beam therapy. Medical Physics, 40(5):051722.

[Rietzel and Chen, 2006] Rietzel, E. and Chen, G. T. Y. (2006). Deformable registration of 4D computed tomography data. Med. Phys., 33(11):4423-4430.

[Rietzel et al., 2005a] Rietzel, E., Chen, G. T. Y., Choi, N. C., and Willet, C. G. (2005a). Fourdimensional image-based treatment planning: Target volume segmentation and dose calculation in the presence of respiratory motion. Int. J. Radiat. Oncol., Biol., Phys., 61(5):15351550.

[Rietzel et al., 2005b] Rietzel, E., Pan, T., and Chen, G. T. Y. (2005b). Four-dimensional computed tomography: Image formation and clinical protocol. Med. Phys., 32(4):874-889.

[Rietzel et al., 2007] Rietzel, E., Schardt, D., and Haberer, T. (2007). Range accuracy in carbon ion treatment planning based on ct-calibration with real tissue samples. Radiat. Oncol., $2(1): 14$.

[Roelofs et al., 2012] Roelofs, E., Engelsman, M., Rasch, C., Persoon, L., Qamhiyeh, S., de Ruysscher, D., Verhaegen, F., Pijls-Johannesma, M. ., Lambin, P., and Consortium, R. O. C. O. C. O. (2012). Results of a multicentric in silico clinical trial (rococo): comparing radiotherapy with photons and protons for non-small cell lung cancer. $J$ Thorac.Oncol., 7(1):165-176.

[Rossi, 2016] Rossi, S. (2016). The national centre for oncological hadrontherapy (cnao): Status and perspectives. Physica Medica: European Journal of Medical Physics, 31(4):333-351.

[RTOG, 2014] RTOG (2014). Rtog 1308 protocol information: Phase iii randomized trial comparing overall survival after photon versus proton chemoradiotherapy for inoperable stage ii-iiib nsclc. 
[Rueckert et al., 1999] Rueckert, D., Sonoda, L. I., Hayes, C., Hill, D. L. G., Leach, M. O., and Hawkes, D. J. (1999). Nonrigid registration using free-form deformations: application to breast mr images. IEEE Transactions on Medical Imaging, 18(8):712-721.

[Rusthoven et al., 2009] Rusthoven, K. E., Kavanagh, B. D., Burri, S. H., Chen, C., Cardenes, H., Chidel, M. A., Pugh, T. J., Kane, M., Gaspar, L. E., and Schefter, T. E. (2009). Multiinstitutional phase $\mathrm{i} / \mathrm{ii}$ trial of stereotactic body radiation therapy for lung metastases. Journal of Clinical Oncology, 27(10):1579-1584.

[Saito et al., 2009] Saito, N., Bert, C., Chaudhri, N., Durante, M., Gemmel, A., Lüchtenborg, R., Schardt, D., and Rietzel, E. (2009). Technical status of the real-time beam tracking system. Report.

[Salama et al., 2012] Salama, J. K., Hasselle, M. D., Chmura, S. J., Malik, R., Mehta, N., Yenice, K. M., Villaflor, V. M., Stadler, W. M., Hoffman, P. C., Cohen, E. E. W., Connell, P. P., Haraf, D. J., Vokes, E. E., Hellman, S., and Weichselbaum, R. R. (2012). Stereotactic body radiotherapy for multisite extracranial oligometastases. Cancer, 118(11):2962-2970.

[Salguero et al., 2011] Salguero, F. J., Saleh-Sayah, N. K., Yan, C., and Siebers, J. V. (2011). Estimation of three-dimensional intrinsic dosimetric uncertainties resulting from using deformable image registration for dose mapping. Medical Physics, 38(1):343-353.

[Sarrut, 2006] Sarrut, D. (2006). Deformable registration for image-guided radiation therapy. Z Med Phys, 16(4):285-297.

[Schardt et al., 2010] Schardt, D., Elsaesser, T., and Schulz-Ertner, D. (2010). Heavy-ion tumor therapy: Physical and radiobiological benefits. Rev. Mod. Phys., 82(1):383.

[Schneider et al., 1996] Schneider, U., Pedroni, E., and Lomax, A. (1996). The calibration of ct hounsfield units for radiotherapy treatment planning. Physics in Medicine and Biology, 41(1):111.

[Scholz and Kraft, 1994] Scholz, M. and Kraft, G. (1994). Calculation of heavy ion inactivation probabilities based on track structure, $\mathrm{x}$ ray sensitivity and target size. Radiat. Prot. Dosim., 52(1-4):29-33.

[Schreibmann et al., 2012] Schreibmann, E., Pantalone, P., Waller, A., and Fox, T. (2012). A measure to evaluate deformable registration fields in clinical settings. Journal of Applied Clinical Medical Physics, 13(5).

[Seeley et al., 2007] Seeley, R., Stephans, T., and Tate, P. (2007). Essentials of Anatomy and Physiology. McGraw-Hill International, Columbus, USA, 6 edition. 
[Seppenwoolde et al., 2002] Seppenwoolde, Y., Shirato, H., Kitamura, K., Shimizu, S., Lebesque, J. V., and Miyasaka, K. (2002). Precise and real-time measurement of 3D tumor motion in lung due to breathing and heartbeat, measured during radiotherapy. Int. $J$. Radiat. Oncol. Biol. Phys., 53(4):822-834.

[Shackleford et al., 2010] Shackleford, J. A., Kandasamy, N., and Sharp, G. C. (2010). On developing b-spline registration algorithms for multi-core processors. Physics in Medicine and Biology, 55(21):6329-6351.

[Shirato et al., 2004] Shirato, H., Seppenwoolde, Y., Kitamura, K., Onimura, R., and Shimizu, S. (2004). Intrafractional tumor motion: Lung and liver. Semin. Radiat. Oncol., 14(1):10-18.

[Shrieve and Loeffler, 2011] Shrieve, D. C. and Loeffler, J. S. (2011). Human Radiation Injury. Lippincott Williams \& Wilkins, Philadelphia, USA.

[Siegel et al., 2014] Siegel, R., Ma, J., Zou, Z., and Jemal, A. (2014). Cancer statistics, 2014. CA: A Cancer Journal for Clinicians, 64(1):9-29.

[Slicer, 2016a] Slicer (2016a). https://www.slicer.org.

[Slicer, 2016b] Slicer (2016b). http://www.slicer.org/publications.

[Socinski et al., 2013] Socinski, M. A., Evans, T., Gettinger, S., Hensing, T. A., Sequist, L. V., Ireland, B., and Stinchcombe, T. E. (2013). Treatment of stage $\{$ IV $\}$ non-small cell lung cancer: Diagnosis and management of lung cancer, 3rd ed: American college of chest physicians evidence-based clinical practice guidelines. Chest, 143(5, Supplement):e341S - e368S.

[Sonke et al., 2008] Sonke, J. J., Lebesque, J., and van, H. M. (2008). Variability of fourdimensional computed tomography patient models. Int. J. Radiat. Oncol. Biol. Phys., 70(2):590-598.

[Stanley et al., 2013] Stanley, N., Glide-Hurst, C., Kim, J., Adams, J., Li, S., Wen, N., Chetty, I. J., and Zhong, H. (2013). Using patient-specific phantoms to evaluate deformable image registration algorithms for adaptive radiation therapy. J Appl Clin Med Phys, 14(6):43634363.

[Takahashi et al., 2014] Takahashi, W., Mori, S., Nakajima, M., Yamamoto, N., Inaniwa, T., Furukawa, T., Shirai, T., Noda, K., Nakagawa, K., and Kamada, T. (2014). Carbon-ion scanning lung treatment planning with respiratory-gated phase-controlled rescanning: simulation study using 4-dimensional ct data. Radiation Oncology, 9:238.

[Tang et al., 2013] Tang, C., Xie, X., and Du, R. (2013). Improved image registration technique using demons and b-spline. Biomedical and Biotechnology Engineering, 3(A). 
[Tashkin, 2013] Tashkin, D. P. (2013). Effects of marijuana smoking on the lung. Annals of the American Thoracic Society, 10(3).

[Taylor et al., 2007] Taylor, R., F., N., and A., D. (2007). Meta-analysis of studies of passive smoking and lung cancer: effects of study type and continent. 36(5).

[Thirion, 1998] Thirion, J. (1998). Image matching as a diffusion process: an analogy with maxwell's demon. Med Image Anal, 2(3):243 - 260.

[Timmerman et al., 2006] Timmerman, R., McGarry, R., Yiannoutsos, C., Papiez, L., Tudor, K., DeLuca, J., Ewing, M., Abdulrahman, R., DesRosiers, C., Williams, M., and Fletcher, J. (2006). Excessive toxicity when treating central tumors in a phase ii study of stereotactic body radiation therapy for medically inoperable early-stage lung cancer. Journal of Clinical Oncology, 24(30):4833-4839.

[Timmerman et al., 2010] Timmerman, R., Paulus, R., Galvin, J., Michalski, J., Straube, W., Bradley, J., Fakiris, A., Videtic, G., Johnstone, D., Fowler, J., Gore, E., and Choy, H. (2010). Stereotactic body radiation therapy for inoperable early stage lung cancer. JAMA, 303(11):1070-1076.

[Trofimov et al., 2005] Trofimov, A., Rietzel, E., Lu, H. M., Martin, B., Jiang, S., Chen, G. T., and Bortfeld, T. (2005). Temporo-spatial imrt optimization: concepts, implementation and initial results. Phys Med Biol, 50(12):2779-2798.

[Trovo et al., 2014] Trovo, M., Minatel, E., Durofil, E., Polasel, J., Avanzo, M., Baresic, T., Bearz, A., Del Conte, A., Franchin, G., Gobitti, C., Rumeileh, I. A., and Trovo, M. G. (2014). Stereotactic body radiation therapy for re-irradiation of persistent or recurrent non-small cell lung cancer. Int J Radiat Oncol Biol Phys, 88.

[Tsao, 2016] Tsao, A. S. (2016). Lung carcinoma. Merck Manual Professional Edition.

[Tsujii and Kamada, 2012] Tsujii, H. and Kamada, T. (2012). A review of update clinical results of carbon ion radiotherapy. Jpn.J.Clin.Oncol., 42(8):670-685.

[Tsujii et al., 2008] Tsujii, H., Kamada, T., Baba, M., Tsuji, H., Kato, H., Kato, S., Yamada, S., Yasuda, S., Yanagi, T., Kato, H., Hara, R., Yamamoto, N., and Mizoe, J. (2008). Clinical advantages of carbon-ion radiotherapy. New J. Phys., 10(7):075009.

[Underner et al., 2014] Underner, M., Urban, T., and Perriot, J. (2014). Cannabis smoking and lung cancer. Revue des Maladies Respiratoires, 31(6).

[Unkelbach et al., 2009] Unkelbach, J., Bortfeld, T., Martin, B. C., and Soukup, M. (2009). Reducing the sensitivity of impt treatment plans to setup errors and range uncertainties via probabilistic treatment planning. Med Phys, 36(1):149-163. 074812MPH[PII]. 
[van de Water et al., 2009] van de Water, S., Kreuger, R., Zenklusen, S., Hug, E., and Lomax, A. J. (2009). Tumour tracking with scanned proton beams: assessing the accuracy and practicalities. Phys. Med. Biol., 54(21):6549-6563.

[Varadhan et al., 2013] Varadhan, R., Karangelis, G., K., K., and Hui, S. (2013). A framework for deformable image registration validation in radiotherapy clinical applications. $J$ Appl Clin Med Phys, 14(1).

[Varma et al., 1977] Varma, M. N., Baum, J. W., and Kuehner, A. V. (1977). Radial dose, LET and $\mathrm{W}$ for 160 ions in N2 and tissue-equivalent gases. Radiat. Res., 70(3):511-518.

[Venugopal et al., 2005] Venugopal, N., McCurdy, B., Hnatov, A., and Dubey, A. (2005). A feasibility study to investigate the use of thin-plate splines to account for prostate deformation. Physics in Medicine and Biology, 50(12):2871.

[Verma et al., 2010] Verma, P., Huanmei, W., Langer, M., Das, I., and Sandison, G. (2010). Survey: Real-time tumor motion prediction for image-guided radiation treatment. Progress in Nuclear Science and Technology, 13(5):24-35.

[Villaruz et al., 2012] Villaruz, L. C., Kubicek, G. J., and Socinski, M. A. (2012). Management of non-small cell lung cancer with oligometastasis. Current Oncology Reports, 14(4):333-341.

[Vinogradskiy et al., 2009] Vinogradskiy, Y. Y., Balter, P., Followill, D. S., Alvarez, P. E., White, R. A., and Starkschall, G. (2009). Verification of four-dimensional photon dose calculations. Medical Physics, 36(8):3438-3447.

[Vtk, 2016] Vtk (2016). https://www.vtk.org.

[WCRF, 2016] WCRF (2016). Worldwide data. http://www.wcrf.org/int/cancer-factsfigures/worldwide-data.

[Westover et al., 2012] Westover, K. D., Seco, J., Adams, J. A., Lanuti, M., Choi, N. C., Engelsman, M., and Willers, H. (2012). Proton sbrt for medically inoperable stage i nsclc. $J$ Thorac Oncol, 7(6):1021-1025. 22551902[pmid].

[Wikipedia, 2016] Wikipedia (2016). commons.wikimedia.org/wiki/file:mlcshape.svg.

[Wilson, 1946] Wilson, R. R. (1946). Radiological use of fast protons. Radiology, 47:487-491.

[Wu et al., 2008] Wu, Z., Rietzel, E., Boldea, V., Sarrut, D., and Sharp, G. C. (2008). Evaluation of deformable registration of patient lung 4dct with subanatomical region segmentations. Medical Physics, 35(2):775-781.

[Yamada et al., 2008] Yamada, Y., Bilsky, M. H., Lovelock, D. M., Venkatraman, E. S., Toner, S., Johnson, J., Zatcky, J., Zelefsky, M. J., and Fuks, Z. (2008). High-dose, single-fraction 
image-guided intensity-modulated radiotherapy for metastatic spinal lesions. International Journal of Radiation Oncology, 71(2):484-490.

[Yoo et al., 2002] Yoo, T. S., Ackerman, M. J., Lorensen, W. E., Schroeder, W., Chalana, V., Aylward, S., Metaxas, D., and Whitaker, R. (2002). Engineering and algorithm design for an image processing api: a technical report on itk-the insight toolkit. Stud Health Technol Inform, 85:586-592.

[Zhang et al., 2015] Zhang, Y., Knopf, A.-C., Weber, D. C., and Lomax, A. J. (2015). Improving 4d plan quality for pbs-based liver tumour treatments by combining online image guided beam gating with rescanning. Physics in Medicine and Biology, 60(20):8141.

[Zhong et al., 2007] Zhong, H., Peters, T., and Siebers, J. V. (2007). Fem-based evaluation of deformable image registration for radiation therapy. Physics in Medicine and Biology, 52(16):4721.

[Zou et al., 2014] Zou, W., Yin, L., Shen, J., Corradeti, M. N., Kirk, M., Munbodh, R., Fang, P., Jabbour, S. K., Simone, C. B., Yue, N. J., Rengan, R., and Teo, B. K. (2014). Dynamic simulation of motion effects in imat lung sbrt. Radiation Oncology, 9:225. 



\section{Lebenslauf}

\section{Persönliche Daten}

Name Kristjan Anderle

Geburtstag 12. März 1986

Geburtsort Jesenice, Slowenien

Nationalität Slowenisch

\section{Universitäre Ausbildung}

seit $02 / 2013$

Mitglied der Helmholtz Graduate School for Hadron and Ion Research (HGS-HIRe)

seit 02/2013 TU Darmstadt

Promotion (Durchführung an der GSI)

10/2005-10/2011 University of Ljubljana, Slowenien

Faculty of Mathematics and Physics

Titel der Diplomarbeit:

Nuclear Magnetic Resonance in Superconducting NaFeAs under High Pressures

\section{Schulische Ausbildung}

09/2001-06/2005 Gymnasium Bezigrad, Ljubljana, Slowenien

09/1992-06/2001 Osnovna sola prof. dr. Josipa Plemlja, Bled, Slowenien 



\section{Acknowledgments}

I would like to extend my sincerest thanks to everybody, who helped me on my journey to completing my PhD. I wish to start by thanking my advisor Christian Graeff for his guidance, patience and extensive help throughout the years. Christoph Bert for first giving me the opportunity to come to GSI and start with the research of this fascinating field. And Marco Durante for taking me under his wing, for his mentorship and invaluable advice.

Anna Eichhorn for the fruitful discussions and for thoroughly reading through my thesis. Matthias Prall for the vital corrections and your help in last, but crucial moments. Daniel Richter and Sebastian Hild, thank you for your help with programing. A special thank you to Till Dettmering for being a first friend I have made in Germany and helping me settle into new environments, both GSI and Germany. Marta Rovituso for the advice on how to actually deliver the thesis. Tobias Brandt and Jens Woelfelschneider from UK Erlangen for their involvement in DIRQA module development. Thank you to everyone in our work group and the entire Biophysics department for treating me as one of your own and helping me feel at home at GSI.

This thesis would not be the same without the things I have learned and the work I was able to do at the Champalimaud Center for The Unknown in Lisbon. A very special thank you to Joep Stroom for relentlessly replying to my e-mails, preparing the patient data and showing me around the Champalimaund Center. I would like to thank the entire staff of the Oncology Department, especially Sandra Vierra, who welcomed me with open hands and made my time spent there an unforgettable experience. Nuno Pimentel for helping me out with his medical expertise. And Carlo Greco for the collaboration and the opportunity to be a part of the Oncology Department of Champalimaud Center for The Unknown.

Special thanks to my family. Thank you parents for helping me get here. I would not have made it without your help and unconditional support. Writing my thesis was much easier with my brother keeping me up in good spirits and being a good support.

And the biggest thank you definitely goes to the two girls who bring the most joy in my life. My daughter tried to wait patiently for me to finish writing, sometimes failing, but always making me laugh. There is not enough place here to express my gratitude to my wife Vanja. Not only did she help me pursue my dreams by moving with me to Germany, she stood by me and was my lighthouse when I was lost. 



\section{Erklärung zur Dissertation}

Hiermit versichere ich, die vorliegende Dissertation ohne Hilfe Dritter nur mit den angegebenen Quellen und Hilfsmitteln angefertigt zu haben. Alle Stellen, die aus Quellen entnommen wurden, sind als solche kenntlich gemacht. Diese Arbeit hat in gleicher oder ähnlicher Form noch keiner Prüfungsbehörde vorgelegen.

Darmstadt, den 15. Juli 2016

(Kristjan Anderle) 
